\title{
Protein kinase D: at the crossroad of cardiac function and metabolism
}

Citation for published version (APA):

Dirkx, E. (2012). Protein kinase D: at the crossroad of cardiac function and metabolism. [Doctoral Thesis, Maastricht University]. BOXPress. https://doi.org/10.26481/dis.20120203ed

Document status and date:

Published: 01/01/2012

DOI:

10.26481/dis.20120203ed

Document Version:

Publisher's PDF, also known as Version of record

\section{Please check the document version of this publication:}

- A submitted manuscript is the version of the article upon submission and before peer-review. There can be important differences between the submitted version and the official published version of record.

People interested in the research are advised to contact the author for the final version of the publication, or visit the DOI to the publisher's website.

- The final author version and the galley proof are versions of the publication after peer review.

- The final published version features the final layout of the paper including the volume, issue and page numbers.

Link to publication

\footnotetext{
General rights rights.

- You may freely distribute the URL identifying the publication in the public portal. please follow below link for the End User Agreement:

www.umlib.nl/taverne-license

Take down policy

If you believe that this document breaches copyright please contact us at:

repository@maastrichtuniversity.nl

providing details and we will investigate your claim.
}

Copyright and moral rights for the publications made accessible in the public portal are retained by the authors and/or other copyright owners and it is a condition of accessing publications that users recognise and abide by the legal requirements associated with these

- Users may download and print one copy of any publication from the public portal for the purpose of private study or research.

- You may not further distribute the material or use it for any profit-making activity or commercial gain

If the publication is distributed under the terms of Article $25 \mathrm{fa}$ of the Dutch Copyright Act, indicated by the "Taverne" license above, 


\section{Protein kinase D: At the crossroad of cardiac function and metabolism}


(c) copyright Ellen Dirkx, Maastricht 2012

Cover Design: Ben Hagenaars

Published by: Uitgeverij BOXPress, Oisterwijk

ISBN: 978-90-8891-371-6 


\title{
Protein kinase D: At the crossroad of cardiac function and metabolism
}

\author{
PROEFSCHRIFT \\ Ter verkrijging van de graad van doctor \\ aan de Universiteit Maastricht, \\ op gezag van de Rector Magnificus, \\ Prof. Mr. G.P.M.F. Mols \\ Volgens het besluit van het college van Decanen, \\ In het openbaar te verdedigen \\ Op vrijdag 3 februari 2012 om 10 uur \\ door

\section{Ellen Dirkx}

Geboren te Geel op 5 mei 1985 


\section{Promotor}

Prof. Dr. Jan F.C. Glatz

\section{Co-promotors}

Dr. Joost J.F.P. Luiken

Dr. Guillaume J.J.M. van Eys

\section{Assessment Committee}

Prof. Dr. Leon de Windt (chairman)

Prof. Dr. Frits Prinzen

Prof. Dr. Felix Mottaghy

Prof. Dr. Ellen Aasum (University of Troms $\varnothing$, Norway)

Prof. Dr. Christoph Beauloye (Université Catholique de Louvain, Belgium)

greiner bio-one

Financial support by the Netherlands Heart Foundation and Diabetes Fonds for the publication of this thesis is gratefully acknowledged. 


\section{CONTENTS}

$\begin{array}{lll}\text { Chapter } 1 \text { Introduction } & 7\end{array}$

Chapter 2 High fat diet induced diabetic cardiomyopathy 25

Chapter 3 Protein kinase D is essential for contraction-induced glucose $\quad 43$

uptake, but is not involved in fatty acid uptake into

cardiomyocytes

Chapter 4 Detection of cardiac myosin binding protein-C, by a phosphospecific PKD antibody in contracting rat cardiomyocytes

Chapter 5 Protein kinase D increases maximal Ca2+-activated tension of cardiomyocyte contraction by selective phosphorylation of cMyBP-C-Ser315

Chapter 6 AMP-activated protein kinase and Protein Kinase D overexpression to restore cardiac insulin resistance

Chapter 7 A protein kinase D1 -MEF2D pathway controls metabolic substrate switching in the failing heart

Chapter 8 General Discussion

Summary

Samenvatting

Dankwoord

Curriculum Vitae 

CHAPTER 1

General introduction 
Cardiomyocytes (heart muscle cells) need a constant supply of ATP to function properly and to meet changes in workload. They require ATP for sarcomeric contraction and relaxation and for membrane transport systems (e.g., $\mathrm{Na}^{+} / \mathrm{K}^{+}$ATPase). ${ }^{1}$ An increase in mechanical activity will raise heart rate and contractility, for which the cardiomyocytes will require more ATP. The size of cellular ATP pools relies on the balance between ATP utilization and ATP production. To synthesize ATP, these cells oxidize long chain fatty acids (LCFA), glucose and to a lesser extent lactate. Under aerobic, non-ischemic conditions, cardiac fatty acid utilization typically accounts for $60-70 \%$ of ATP production, glucose covers $\sim 20-30 \%$ of needs and lactate covers $\sim 10 \% .{ }^{2}$ In the healthy heart this substrate utilization can vary between certain limits. However, over-utilization of one of the two major substrates appears to be associated with hypertrophy, contractile dysfunction and eventually heart failure. $^{3-5}$

In diabetic hearts, insulin fails to stimulate cardiac glucose uptake, which leads to enhanced LCFA utilization. ${ }^{5,6}$ In the long run this results in LCFA becoming almost the exclusive energy source for the diabetic heart. Studies in diabetic patients and animal models have shown that increased LCFA uptake, at the expence of glucose uptake, is accompagnied by hypertrophic remodeling with consequences as described above (further discussed in chapter 2 ). ${ }^{7}$ Also, an increased glucose uptake has been associated with hypertrophic remodeling and heart failure. ${ }^{8}$ Thus, alterations in cardiac substrate utilization, whether increased LCFA uptake or increased glucose uptake, seems to be related to cardiac remodeling. This indicates that a balanced substrate uptake is crucial for normal cardiac morphology and function. ${ }^{9}$

Understanding the pathways that regulate the cardiac substrate balance is crucial to identify therapeutic targets in chronic diseases such as diabetic cardiomyopathy. This thesis describes a new pathway involved both in contractile function and cardiac substrate uptake. The pathway centers on the intracellular kinase, protein kinase $D$, which during increased contraction stimulates both glucose uptake and contractile capacity of the heart muscle.

\section{Cardiac contractile function}

The heart cycles continuously through systolic contraction and diastolic relaxation. The contraction of the heart is regulated beat-to-beat by calcium, stored and released from the sarcolemma of cardiomyocytes, in a process called excitationcontraction coupling. ${ }^{10}$ Cardiomyocytes use interactions (cross-bridges) between actin and myosin to develop force. ${ }^{1}$ These crossbridges are coupled to a concomitant series of chemical reactions involving the hydrolysis of ATP to ADP and inorganic phosphate. When ATP is bound to the myosin head, it immediately causes a change in the conformation of the domains that make up the actin-binding site. This reduces the affinity of the myosin head for actin and allows it to move along 
the filament. When hydrolysis of ATP occurs, ADP and inorganic phosphate (Pi) remain bound to the myosin. A weak binding of the myosin head to a new site on the actin filament causes release of the $\mathrm{Pi}$, which results in the transition from weak to strong binding of the myosin head to the actin filament. This triggers the force generating change in shape of the myosin head to regain its original conformation, and is called the power stroke. Thus, force production is thought to be coupled to the release of $\mathrm{Pi}$ and comprises the transition from weak to strong binding states. When the myosin head loses its bound ADP the cross-bridge cycle is terminated, and a new contraction cycle will start. Upon binding of new ATP, the cross-bridge dissociates from actin and the cycle is ready to start again. ${ }^{1,11}$

The cross-bridges are initiated by changes in cytoplasmic $\mathrm{Ca}^{2+}$ concentrations and the responsiveness of myofilaments to $\mathrm{Ca}^{2+}$. During the depolarizing phase of the cardiac action potential, $\mathrm{Ca}^{2+}$ enters the cell through voltage-activated $\mathrm{L}$ - type $\mathrm{Ca}^{2+}$ channels and triggers ryanodine receptors (RyR type 2 isoform) to open and release $\mathrm{Ca}^{2+}$ from sarcoplasmic reticulum (SR) $\mathrm{Ca}^{2+}$ stores. This $\mathrm{Ca}^{2+}$-induced $\mathrm{Ca}^{2+}$ release will increase intracellular $\mathrm{Ca}^{2+}$ levels and activate proteins of the contractile apparatus to trigger cardiac contraction. Binding of $\mathrm{Ca}^{2+}$ to troponin-C $(\mathrm{TnC})$ will lead to structural changes in contractile proteins and changes in protein-protein interactions. These events are necessary for force generation of cross-bridges in systolic contraction. The reversal of these events leads to diastolic relaxation. ${ }^{1,12}$

Cardiac troponin is located at regular intervals along the thin filament and consists of three subunits: cardiac troponin C (cTnC), troponin I (cTnl), and troponin T (cTnT). Cardiac $\mathrm{Tnl}$ is the inhibitory subunit, primarily functioning to prevent actin and myosin from interacting in the absence of $\mathrm{Ca}^{2+}$. The binding of actin in low $\mathrm{Ca}^{2+}$ and the alternative binding of $\mathrm{TnC}$ in the presence of high $\mathrm{Ca}^{2+}$ by the inhibitory region of $\mathrm{Tnl}$, provides a $\mathrm{Ca}^{2+}$ sensitive mechanism for inhibition of muscle contraction. As the intracellular $\mathrm{Ca}^{2+}$ concentration increases, $\mathrm{Ca}^{2+}$ will bind to $\mathrm{TnC}$, which causes conformational changes of the tropinin subunits and a decreased interaction between $\mathrm{Tnl}$ and actin. These conformational changes allow movement of tropomyosin along the actin filament, which facilitates the interaction between myosin and actin, leading to muscle contraction. The $\mathrm{Ca}^{2+}$ sensitivity of the muscle to contract is affected by phosphorylation of $\mathrm{Tnl}$ (Ser 22 and 23) which can be phosphorylated by protein kinase $A(P K A)$, protein kinase $C$ (PKC) and protein kinase $\mathrm{D}$ (PKD). ${ }^{13-15}$

Cardiac myosin binding protein-C (cMyBP-C) is a protein associated with the thick filament, which localizes to the crossbridge containing $C$ zones of the sarcomere, through interactions with specific regions of titin and myosin (Figure 1.1). ${ }^{16}$ It consists of immunoglobulin (IgC2-like) and fibronectin (FN3) domains. ${ }^{17}$ cMyBP-C acts as a physical restrain on the myosin heads, keeping them near the thick filament backbone and thus reducing the probability of their interaction with actin during diastole. ${ }^{17}$ The absence of CMyBP-C results in eccentric left ventricular hypertrophy, myocardial disarray, interstitial fibrosis and diastolic dysfunction in 
homozygous knock-out or knock-in mice. ${ }^{16,18}$ Untill now, in mice there are four phosphorylation sites of CMyBP-C known: Ser281, Ser290, Ser310, and Ser315. Upon $ß$-adrenergic stimulation phosphorylation of cMyBP-C is regulated by cAMPdependent protein kinase (PKA), by intracellular $\mathrm{Ca}^{2+}$ levels via the $\mathrm{Ca}^{2+} /$ calmodulin kinase II (CaMKII), by protein kinase $C \varepsilon(P K C \varepsilon)$ in a $\mathrm{Ca}^{2+}$-independent manner and by PKD. Phosphorylation of CMyBP-C plays a role in regulating force generation. ${ }^{17}$ It is generally accepted that CMyBP-C phosphorylation plays a major role in the acceleration of cross-bridge kinetics. ${ }^{19}$

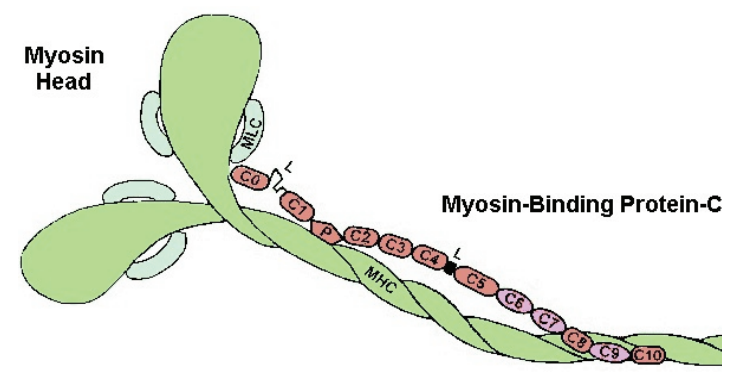

Figure 1.1: Position of $c M y B P-C$ in association with MHC (adapted from Witt et al, 2001). ${ }^{16}$ cMyBP-C interacts with both the rod portion and the neck region of the myosin molecule. Phosphorylation of MyBP-C releases CMyBP-C from the myosin neck. Interaction of the cardiac-specific CO domain of MyBP-C with the myosin head would constrain cross-bridge movement. $M L C$, myosin light chain; $M H C$, myosin heavy chain;CO-C10, cMyBP-C domain numbers; $P$, MyBP-C motif; $L$, linker region.

\section{Cardiac substrate uptake and utilization}

The heart possesses a limited storage capacity for LCFA and glucose and therefore LCFA and glucose are supplied mainly and continuously from extracellular sources. ${ }^{20}$ Because the heart is a metabolically flexible organ, it can use both LCFA and glucose to oxidatively produce ATP. Depending on substrate availability, either LCFA metabolism or glucose metabolism is increased. So, when increased amounts of LCFA are circulating in the blood, the heart will increase LCFA oxidation and reduce glucose oxidation. Therefore, substrate availability is one of the primary factors that determine substrate preference. ${ }^{21}$ To be taken up by the cardiomyocytes, LCFA and glucose need to pass the selectively permeable lipid bilayer of the cardiomyocytes.

\section{LCFA uptake and utilization}

LCFA can be taken up by passive diffusion (20\%). Accumulation of LCFA close to the cell surface will create a local diffusion gradient across the membrane to facilitate the flip-flop of LCFA across the bilayer (passive diffusion). The main way to take up LCFA is by protein-mediated transport $(80 \%) .{ }^{22}$ The protein-mediated transport of 
LCFA depends largely on the plasma membrane transporter CD36 and plasmalemmal fatty acid-binding protein (FABPpm). ${ }^{23} \mathrm{CD} 36$, also known as fatty acid translocase (FAT) is a $88 \mathrm{kDa}$ heavily glycosylated integral membrane protein, and has two transmembrane domains, two short cytoplasmic tails and an extracelular domain. ${ }^{7,24}$ This structure makes it likely that CD36 functions mainly by trapping of LCFA to the plasma membrane. The transmembrane protein CD36 and the peripheral membrane protein $\mathrm{FABP}_{\mathrm{pm}}$ could act as acceptors for fatty acids to increase their concentration at the cell surface and thus enhance the number of fatty acid-diffusion events. ${ }^{7}$ It was shown that CD36 and FABPpm most likely act in concert to mediate LCFA uptake by cardiomyocytes. ${ }^{25}$ However, it also might be that CD36 traps LCFA to the plasma membrane to supply fatty acid transport proteins (FATP) with LCFA which in turn transfer this substrate directly across the bilayer through a channel formed by complexes of membrane-associated proteins (facilitated diffusion). ${ }^{7}$ Alternatively, CD36 itself may also facilitate the transport of fatty acids across the phospholipid bilayer (uptake by facilitated diffusion). ${ }^{7}$ In the cytoplasm LCFA bind to cytosolic fatty acid-binding protein $\left(F A B P_{c}\right)$ after which they are transported through the cytoplasm to the mitochondria, and will enter $\beta$ oxidation to generate ATP or will be stored as triacylglycerol (see chapter 2 ).

\section{Glucose uptake and utilization}

Transport of glucose over the plasma membrane occurs along a steep concentration gradient. Because of its hydrophilic nature, glucose is unable to pass the lipid bilayer of the plasma membrane by passive diffusion, therefore the transport of glucose is regulated by specific transporters. ${ }^{26}$ Glucose uptake into the heart is entirely mediated by members of the glucose transporter (GLUT) family. ${ }^{27-29}$ At present, thirteen members of this GLUT family are known. ${ }^{30,}{ }^{31}$ In the heart, mainly two members of the GLUT family have been found, GLUT1 and GLUT4. ${ }^{32,}{ }^{33}$ GLUT1 is resposible for basal glucose uptake, while GLUT4 facilitates glucose uptake upon insulin stimulation or elevated contractile activity. ${ }^{26,34}$ Both transporters have a $\mathrm{Km}$ for glucose (concentration of glucose at which the rate of transport is half-maximal) that is in the range of plasma glucose concentrations under fasting conditions. ${ }^{35}$ In addition to their presence at the plasma membrane, these transporters also have been found in intracellular compartments. ${ }^{26,36}$ These intracellular compartments are known as endosomes and function as storage depots. During stimulation, the transporters are directed from the intracellular stores to the plasma membrane, resulting in an increased capacity of glucose transport. ${ }^{26}$ This transporter translocation is a vesicle-mediated proces. ${ }^{37,38}$

After transportation across the plasma membrane, intracellular glucose is rapidly phosphorylated by hexokinase. ${ }^{39}$ Glucose-6-phosphate mainly will enter the glycolytic pathway, where glucose is ultimately broken down to pyruvate, which, in turn, is a substrate for further metabolism. However, glucose-6-phosphate is also a substrate for glycogen synthesis and ribose synthesis. ${ }^{39}$ 


\section{Pathways that direct substrate uptake}

In the heart, insulin and contraction are the two main physiological stimuli that increase substrate uptake. ${ }^{37}$ Although both stimili simultaneously induce LCFA and glucose uptake, their signaling pathways differ markedly.

\section{The insulin signaling pathway}

Insulin is the regulatory hormone for whole-body glucose homeostasis. Circulating insulin stimulates glucose and LCFA uptake into cells (Figure 1.2). Insulin-stimulated substrate uptake is initiated by binding of insulin to the $\alpha$-subunit of the insulin receptor at the sarcolemma. ${ }^{40}$ This induces a conformational change resulting in the autophosphorylation of a number of tyrosine residues present in the $\beta$-subunits. ${ }^{41}$ Tyrosine autophosphorylation leads to activation of the receptor's intrinsic tyrosine kinase activity, which results in phosphorylation of the two insulin receptor substrates IRS-1 and IRS-2. Phosphorylated IRS1/2 then binds and activates the regulatory subunit of phosphotidylinositol 3-kinase (PI3K) to produce phosphatidylinositol 3,4,5-triphosphate. This will activate phosphoinositidedependent protein kinase B/Akt (PKB/Akt), 3-phosphoinositide-dependent kinase-1 (PDK1), as well as the atypical protein kinase $C$ isoforms $\zeta$ and $\lambda{ }^{40,42,43}$ Activated PKC- $\lambda / \zeta$ will lead to translocaion of GLUT4 from endosomal pools to the plasma membrane to take up glucose. ${ }^{44,45}$ Additionaly, activated Akt phosphorylates and inhibits its $160 \mathrm{kDa}$ substrate (AS160), thereby inducing both GLUT4 and CD36 vesicles to translocate from the intracellular pools to the sarcolemma ${ }^{46}$ Vesicle destination depends on the docking protein. One such protein involved in vesicle targeting is soluble $\mathrm{N}$-ethylmaleimide-sensitive factor attachment protein receptor (SNARE). ${ }^{47}$ SNAREs can be classified into two categories: vesicle SNARE (v-SNARE), which is incorporated into the membranes of transport vesicles, and target SNARE (t-SNARE), which is located in target membranes. ${ }^{48}$ Following specific interaction between these two SNAREs, vesicles are able to dock onto target membranes. Trafficking of GLUT4 to the plasma membrane in response to insulin is regulated by several v-SNARE proteins like vesicle-associated membrane protein-2 (VAMP-2). ${ }^{37,47}$ VAMP2 helps to guide the vesicles to the docking site on the plasma membrane, were the tSNAREs synaptosomal-associated membrane protein of $23 \mathrm{kDa}$ (SNAP-23) and syntaxin are located. ${ }^{49}$ Besides GLUT4, also CD36 trafficking is mediated by VAMP proteins during insulin stimulation. ${ }^{37}$ 


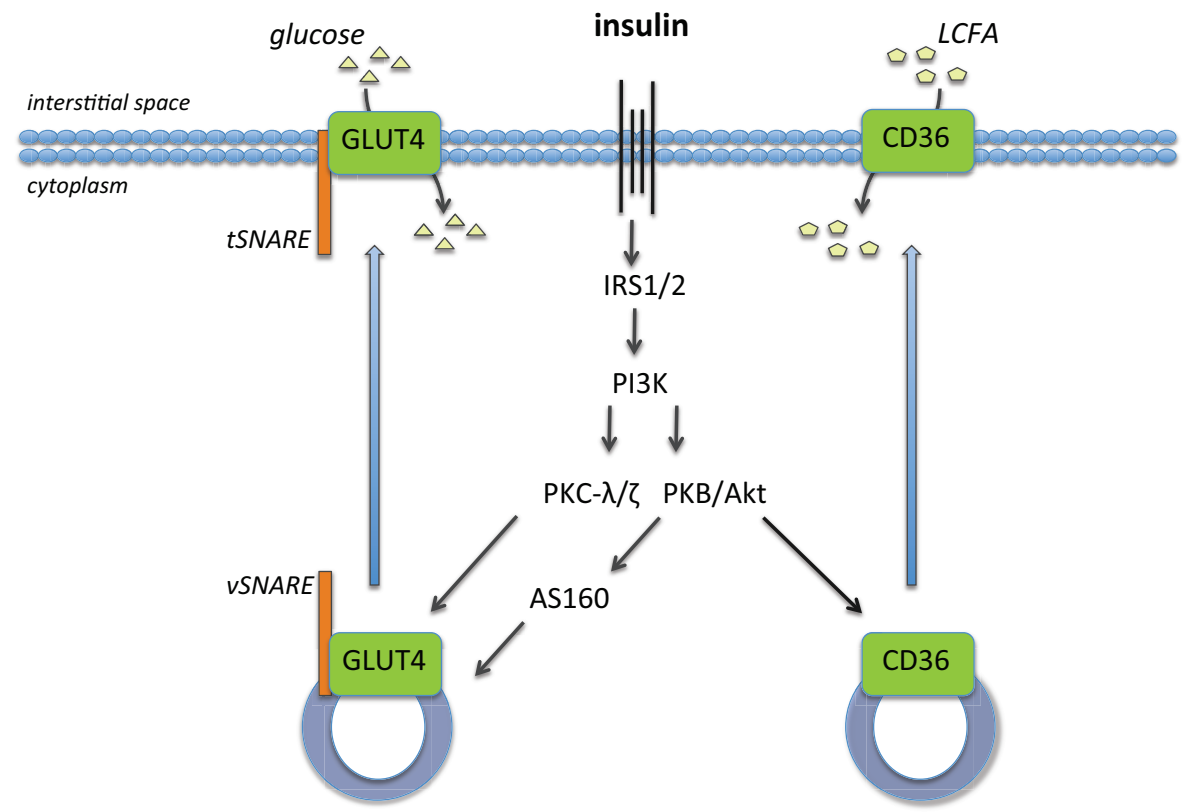

Figure 1.2: Insulin signaling pathway: Effect on glucose and LCFA uptake. During insulin stimulation, the two insulin receptor substrates IRS-1 and IRS-2 become phosphorylated. Phosphorylated IRS1/2 then binds and activates phosphotidylinositol 3-kinase (PI3K). This will lead to the production of phosphatidylinositol 3,4,5-triphosphate, and activate protein kinase B/Akt (PKB/Akt) and the atypical protein kinase $C$ isoforms $\lambda$ and $\zeta$ (PKC- $\lambda / \zeta$ ). Activated Akt phosphorylates and inhibits its $160 \mathrm{kDa}$ substrate (AS160), thereby inducing GLUT4 and CD36 vesicles to translocate from the intracellular pools to the sarcolemma. GLUT4 trafficking to the plasma membrane, in response to insulin, is regulated by several v-SNARE proteins. These proteins help to guide the vesicles to the docking site on the plasma membrane, were the tSNAREs are located.

\section{The contraction signaling pathway}

The continuous contraction of cardiomyoctes requires the production of large amounts of ATP, which is primarily met by using LCFA and glucose. Because of this continuous contraction of cardiomyocytes, both GLUT4 and CD36 uninteruptedly cycle between the intracellular compartments and the plasma membrane to take up glucose and LCFA for the generation of ATP. ${ }^{50}$ One of the proteins involved in the contraction signaling pathway is AMP kinase (AMPK). ${ }^{51-53}$ It has been shown that AMPK activation leads to translocation of both GLUT4 as CD36 to the plasma membrane. ${ }^{54}$ Next to AMPK, also PKC and PKD have been shown to be activated during contraction (Figure 1.3). ${ }^{55}$ In the next paragraph we will describe these kinases in more detail. 


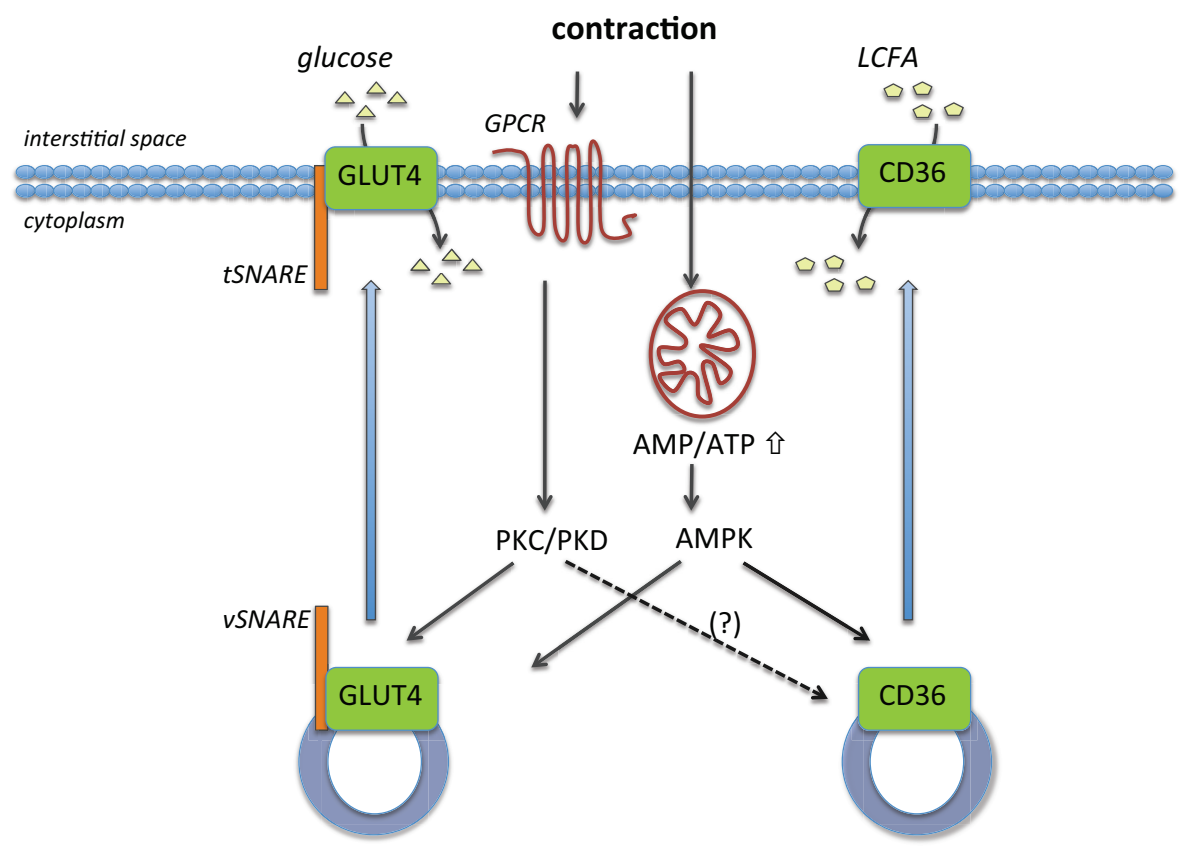

Figure 1.3: Contraction signaling pathway: Effect on glucose and LCFA uptake. During contraction, the AMP/ATP ratio will increase, which leads to the activation of AMP-kinase (AMPK). Activated AMPK induces translocation of both GLUT4 and CD36 from the endosomal pools to the plasma membrane. Also, G-protein coupled receptors (GPCR) will be stimulated. This will lead to the activation of signaling cascades, which involve protein kinase $C$ and D (PKC and PKD). Activation of both PKC and PKD will lead to the translocation of GLUT4 from endosomal pools to the plasma membrane to take up glucose. Whether PKC and PKD play a role in translocating CD36 to the plasma membrane remains to be elucidated.

\section{Kinases directing glucose and fatty acid uptake}

\section{AMP kinase}

AMP kinase (AMPK) is a heterotrimeric protein consisting of a catalytic $\alpha$ subunit and two regulatory $\beta$ and $\gamma$ subunits. ${ }^{56}$ Different isoforms of each subunit have been identified. In the heart, both the $\alpha 1$ and $\alpha 2$ subunits are expressed, but $\alpha 2$ is the predominant subunit. Generally, AMPK activation results in activation of catabolic pathways and inhibition of anabolic pathways (e.g. substrate storage). Activation of AMPK occurs through phosphorylation by upstream kinases, such as LKB1 or by binding of AMP to one of the regulatory subunits. ${ }^{56}$ Next to its role in metabolism, a recent publication showed that AMPK also phosphorylates $\mathrm{Tnl}$ in both skeletal and cardiac muscle. ${ }^{57}$ This implicates that AMPK plays a role in regulating muscle 
contractility and therefore AMPK possibly can link metabolism with increased contraction.

\section{Protein kinase $\mathrm{C}$}

Protein kinase $\mathrm{C}$ (PKC) belongs to a serine-threonine protein kinase family and consists of three subgroups; (1) conventional $(\alpha, \beta \mathrm{I}, \beta \mathrm{II}, \gamma),(2)$ novel $(\delta, \varepsilon, \eta, \theta)$ and (3) atypical $(\zeta, \lambda)$ PKCs. PKC $-\mu$ is not mentioned here because this kinase is now classified as PKD, and will be discussed in the next paragraph. All PKC subgroups comprise a C-terminal serine/threonine protein kinase domain linked through a variable ' $\mathrm{V} 3$ ' domain to a regulatory domain (Figure 1.4). The regulatory domain consists of the $\mathrm{C} 1$ and $\mathrm{C} 2$ domains, and variable regions (V) 1-3. The signal transduction pathway that activates PKC consists of receptor-mediated activation of phospholipases C, leading to hydrolysis of phosphatidylinositol-4,5-diphosphate to produce diacylglycerol (DAG), as well as a rise in intracellular calcium $\left(\mathrm{Ca}^{2+}\right)$ levels. PKC isozymes are highly homologous in their catalytic domain, but their regulatory domains determine the response of individual members to activators. Conventional PKCs are DAG and $\mathrm{Ca}^{2+}$ dependent enzymes. On the other hand novel PKCs require DAG but not $\mathrm{Ca}^{2+}$ for activity, and atypical PKCs are not activated by DAG nor $\mathrm{Ca}^{2+}$, but are stimulated by other lipid-derived second messengers. Inactive PKCs are found in the soluble fraction of the cells. Upon activation, PKCs will translocate to various membrane surfaces, where they bind anchoring molecules, such as AKAP, and phosphorylate neighboring protein substrates ${ }^{58}$.

All PKC isoforms have been found to be present in the heart. However the concentration of the isoforms differs between the atria and ventricles: i.e., the $\mathrm{Ca}^{2+}$ dependent isozymes, $\alpha, \beta \mathrm{I}$, and $\beta \mathrm{II}$, reside predominantly in the ventricle, PKC- $\delta$ and $-\zeta$ are mainly expressed in the atria and PKC- $\varepsilon$ and $-\lambda$ are present in both atria and ventricles. The functional relevance of this distribution is still unknown.

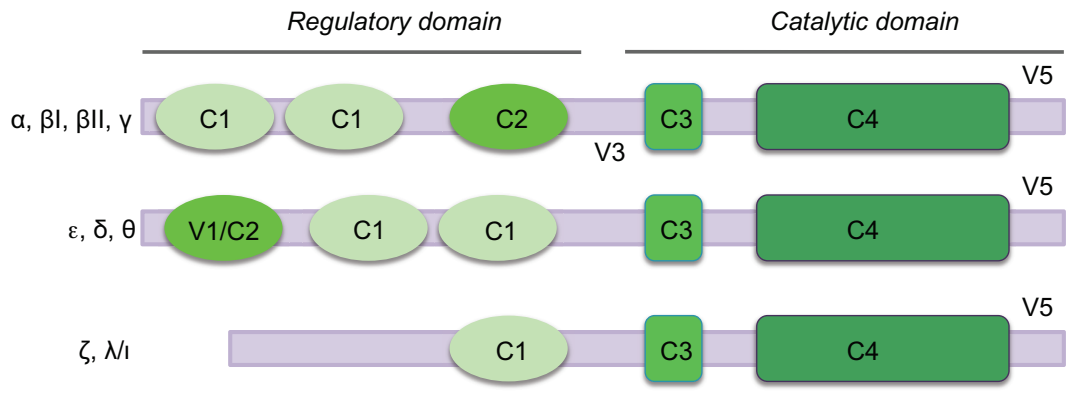

Figure 1.4: Classification and structure of different isoforms of PKC. The PKC family of isoforms consists of three classes: the classical $\left(\alpha, \beta_{1}, \beta_{\|}, \gamma\right)$, novel $(\varepsilon, \delta, \theta)$, and atypical $(\zeta, \lambda / \iota)$. The regulatory domain consists of the $\mathrm{C} 1$ and $\mathrm{C} 2$ domains, and variable regions (V) 1-3. The classical and novel isoforms contain a duplicate of the $\mathrm{C} 1$ domain that binds DAG and its analogs, whereas the atypical family contains only one 
C1 copy. The classical and novel families contain a C2 domain, which binds to phosphatidylserine; the classical C2 binds phosphatidylserine in a calcium-dependent manner. The catalytic domain consists of the ATP binding domain C3 and substrate binding/catalytic domain C4. The C-terminus of the protein contains the V5 domain, which contains some of the phosphorylation sites that regulate PKC activity. (adapted from Wang, 2006) ${ }^{59}$

\section{Role of the PKC in regulating muscle metabolism}

In skeletal myotubes, insulin is able to induce tyrosine phosphorylation and translocation of PKC $\delta$ to the plasma membrane and increases the activity of this isoform, as shown by selective pharmacological blockade and by overexpression of wildtype and point-mutated inactive PKC $\delta$ isoforms. ${ }^{60}$ Selective blockade of PKC $\delta$ by rottlerin inhibited insulin-induced translocation of GLUT4, and significantly reduced the stimulation of glucose uptake by insulin. ${ }^{60}$ These data indicate that this novel PKC isoform induces GLUT4 traffic in muscle cells. However, for cardiac muscle, the role of PKC in insulin-stimulated substrate uptake is still under debate. In isolated cardiomyocytes translocation of CD36 and GLUT4 to the sarcolemma was

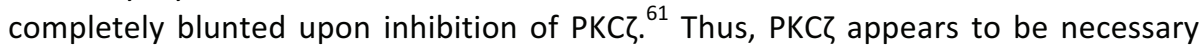
for insulin-induced translocation of GLUT4 and CD36. However, PKC $\zeta$ is already fully active under basal conditions and not further activated by insulin, indicating that its role in insulin-stimulated uptake of both glucose and LCFA is permissive rather than regulatory. ${ }^{61}$ In contrast, Russ et al. showed in isolated ventricular cardiomyocytes that insulin was unable to induce a redistribution of PKC. ${ }^{62}$ Furthermore, in this latter study, PKC inhibitors did not interfere with glucose transport stimulation by insulin. ${ }^{62}$ These puzzling data show that further research needs to be done to elucidate the role of PKC on substrate uptake in insulin-stimulated cardiomyocytes.

Muscle contraction has been shown to elevate both DAG and phosphatidic acid, and activate PKC signaling, ${ }^{63,64}$ which suggests a role for this protein kinase family in contraction-induced changes in muscle metabolism. Phorbol 12-myristate 13acetate (PMA), a cell-permeable DAG analogue, frequently used to pharmacologically activate the conventional and novel PKC isoforms, was shown to stimulate both glucose and LCFA uptake in rat cardiac myocytes. ${ }^{64}$ Interestingly, in the presence of specific PKC inhibitors, AMPK activation does not result in substrate transporter translocation, and therefore, some PKC isoforms might be involved in contraction-induced translocation of both GLUT4 and CD36 in cardiomyocytes (as a potential downstream target of AMPK). ${ }^{64}$ Also, in contracting $\mathrm{C} 2 \mathrm{C} 12$ myotube cultures, cell surface carbachol-induced translocation of GLUT4 was partly inhibited by the conventional/novel PKC inhibitors GF-109203X, Gö6983, and Ro-31-8425. ${ }^{65}$ Thus, PKCs are activated during contraction and induce substrate uptake. 


\section{Role of PKC in regulating cardiac contractility}

The functional consequences of PKC-dependent phosphorylation of the contractile proteins have been suggested to range from changes in mechanical integrity of the cardiac sarcomere to decreased actin-myosin ATPase activity and force generation. ${ }^{66}$ Upon activation, especially the PKC- $\alpha$ and PKC- $\varepsilon$ isoforms are known to translocate to the myofilaments to directly phosphorylate $\mathrm{Tnl}$ and $\mathrm{cMyBP}^{\mathrm{C}} \mathrm{C}^{67}$ Phosphorylation of Tnl-Ser42 and -Ser44 influences the maximum tension of myofilaments and phosphorylation of Tnl-Thr142 reduces the affinity of the inhibitory peptide for calcium saturated Troponin C (TnC). ${ }^{68,69}$ PKC induced cMyBP-C phosphorylation is believed to accelerate cross-bridge cycling and to increase both the rate and strength of contraction. ${ }^{70}$

\section{Protein kinase D}

Protein kinase $D(P K D)$, previously classified as $P K C-\mu$, is closely related to novel and conventional members of the protein kinase $C(P K C)$ family, with which it shares the ability to be activated by diacylglycerol (DAG). ${ }^{59}$ PKD consists of an $\mathrm{N}$-terminal regulatory domain and a $\mathrm{C}$-terminal kinase domain. The $\mathrm{N}$-terminus starts with a non-polar region, rich in alanine and/or proline residues, the second part consists of two cysteine-rich $\mathrm{Zn}$ fingers and an auto-inhibitory pleckstrin homology $(\mathrm{PH})$ domain (Figure 1.5). The catalytic domain of PKD is more closely related to that of the $\mathrm{Ca}^{2+}$ calmodulin-regulated protein kinases (CaMKs) and displays relatively little homology to the catalytic domains of the PKC family. Also, in contrast to members of the PKC family, PKD lacks a pseudosubstrate region. Therefore, PKD has been positioned into a separate kinase family. ${ }^{59}$

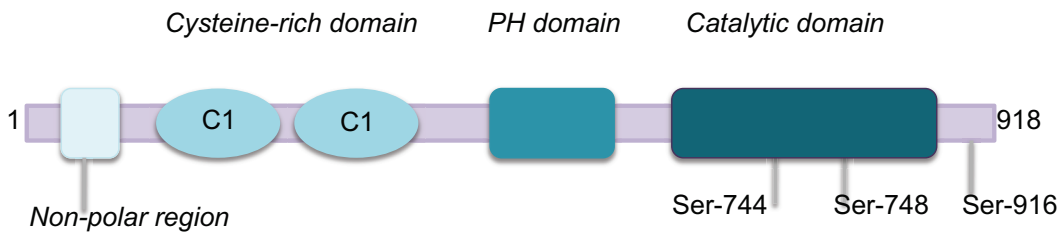

Figure 1.5 Structure of PKD PKD consists of an N-terminal regulatory domain and a C-terminal kinase domain. The $\mathrm{N}$-terminal regulatory domain consists of a non-polar region, two cysteine-rich $\mathrm{Zn}$ fingers and an auto-inhibitory pleckstrin homology $(\mathrm{PH})$ domain. The catalytic domain contains the transphosphorylation sites Ser-744 and Ser-748 and the autophosphorylation site Ser916.

The protein kinase D (PKD) family consists of three members, i.e., PKD1 (formerly classified as PKC $\mu$ ), PKD2, and PKD3, which have overlapping functions ${ }^{59}$. Of these three isoforms PKD1 has been the most intensely studied. Within PKD, the cysteinerich domains have been shown to have a critical role in recruiting and binding PKD to the Golgi apparatus whereas the PH domain has an inhibitory effect on the 
catalytic domain. Although the PH domain is known to bind phosphoinositides, PKD is unable to generate PI3-kinase signals. Several phosphorylation sites have been identified; Ser203 and Ser255 both in the Zn finger linker region, Tyr463 in the PH domain, Ser744 and Ser748, which are both situated in the activation loop, and Ser916, which is located at the carboxy-terminus. ${ }^{71,}{ }^{72}$ Recently, Ser412 has also been identified as a PKD phosphorylation-site. This PKD-Ser412 has been shown to be phosphorylated upon PKC activation and this seems to happen during exposure to hypertrophic agonists (NE and endothelin). ${ }^{73}$ In addition, PKD1 and PKD2 contain a PDZ-binding motif at their C-terminus, allowing proteins to anchor to the actin cytoskeleton.

\section{Activation of PKD}

When PKD is inactive, its two-cysteine and PH residues on the regulatory domain inhibit the catalytic domain. Following initial activation through phosphorylation of Ser 744 and 748, the catalytic domain is uncovered from these inhibitory residues, leading to full activation. In most cases, PKD activation is triggered by binding of DAG. $^{74,75}$ DAG can stimulate PKD directly by binding to its Cys1 domain or indirectly via various $\mathrm{PKC}$ isoforms (PKC $\varepsilon, \delta, \eta, \theta$ ), which can phosphorylate Ser744 and Ser748 within the activation loop of PKD. Trans-phosphorylation of Ser744/748 induces autophosphorylation of Ser916, which has been found to correlate with catalytic activity. ${ }^{76}$ The transient nature of the PKD interaction allows the passage of many molecules through the activation complex. Activated PKD, which is e.g. recruited to membranes, can then also translocate to other cellular sites of action. It has been suggested that A-kinase anchoring proteins (AKAPs) are involved in this process. ${ }^{77}$ AKAPs are signal-organizing molecules that compartmentalize various enzymes that are regulated by second messengers (e.g. the cyclic-AMP-dependent protein kinase (protein kinase A (PKA)). ${ }^{78}$ Interestingly, AKAP is also known to interact with PKD, with the AKAP anchored PKD complex regulating PKD activity. ${ }^{77} \mathrm{~A}$ longer transcript of the Lbc gene, which function as an AKAP, so-called AKAP-Lbc, contributes to PKD stimulation in two ways. ${ }^{79}$ First, it recruits the upstream activating kinase $P K C \eta$ and positions it in close proximity to PKD, and it might provide access to DAG, a crucial lipid activator of both PKC $\eta$ and PKD. Second, AKAP-Lbc directs its own PKAmediated phosphorylation at Ser2737. Thus, on recruitment of PKA to the anchoring protein, PKD activation is made possible through PKA-mediated phosphorylation of Ser 2737 on AKAP. This phosphorylation dissociates PKD from AKAP, releasing PKD into the cytoplasm. Once released, PKD can be stimulated by a number of signals and can shuttle to the nucleus, Golgi, cell surface membranes or mitochondria. ${ }^{79}$

Another way to activate PKD includes mitochondria generated reactive oxygen species (mROS). ${ }^{80}$ Accumulation of mROS causes a translocation of PKD to mitochondria and additional tyrosine phosphorylations in the $\mathrm{PH}$ domain mediated by the tyrosine kinases Src and Abl. ${ }^{81}$ Phosphorylation of the Tyr463 in the PH domain facilitates conformational changes that remove auto-inhibition, which leads 
to full catalytic activity of PKD. The mechanism of how PKD interacts with the mitochondria in response to oxidative stress is an open question. PKD lacks a known mitochondrial targeting sequence that allows import into the mitochondrial matrix. Therefore, it is most likely that PKD localizes to the outer mitochondrial membrane, mediated through lipid or protein binding. Alternatively, PKD could be recruited to the mitochondria via an adaptor protein. Mitochondrial adapters have been described for other kinases that do not possess a mitochondrial targeting sequence such as Src, which can interact with the mitochondria through binding of Dok-4. ${ }^{82}$ For PKD several potential mitochondrial adapters are available. Mitochondrial PKD co-localizes and co-immunoprecipitates with p32, a ubiquitously expressed mitochondrial protein that also binds with high affinity to PKC $\delta$, one of the upstream kinases for PKD. Although p32 could act as a mitochondrial adapter for PKD1, the role of the interaction between $\mathrm{p} 32$ and PKD remains undefined. ${ }^{83,84}$ Another potential mitochondrial adapter could be AKAP. Mitochondrial-localized AKAP protein might serve as a PKD1 and PKC $\delta$ binding protein. ${ }^{77}$ Because the direct upstream kinases of PKD1, PKC $\delta$ and Abl have no mitochondrial targeting sequence and are all located in the mitochondria in response to oxidative stress, one could speculate that there is a joint adaptor which enables the formation of a mitochondrial oxidative stress-sensing PKD1 activation complex.

\section{Function of PKD}

Once localized in different subcellular compartments, PKD carries out various functions. In the cytosol, PKD downregulates c-Jun N-terminal kinase (JNK) activity by phosphorylating c-Jun, ${ }^{85}$ whereas it upregulates ERK1/2 by phosphorylating the Ras- binding protein RIN1. ${ }^{86}$ At the contractile apparatus, PKD phosphorylates cardiac troponin I (cTnl) at $\operatorname{Ser}(22) / \operatorname{Ser}(23)$, which reduces myofilament $\mathrm{Ca}^{2+}$ sensitivity, and it accelerates cross-bridge cycle kinetics by phosphorylation of CMyBP-C. ${ }^{13}$ However, the functional coherency between all these PKD-mediated changes are poorly understood.

In the nucleus, activated PKD can phosphorylate histone deacetylase 5 (HDAC5) that is a known repressor of myocyte enhancer factor 2 (MEF2). ${ }^{87}$ Phosphorylation of HDAC5 promotes its interaction with 14-3-3. This complex is exported from the nucleus to facilitate MEF2 transcription, which stimulates cardiac hypertrophy and GLUT4 expression. ${ }^{87,88}$

At the Golgi apparatus (Golgi), PKD is present and is involved in vesicle formation, whereafter these vesicles are transported to the plasma membrane. ${ }^{89}$ This process is not clearly understood, and several mechanisms have been proposed. The cysteine domains on PKD have been shown to be essential for its recruitment and binding to the Golgi. On binding to Golgi, the vesicle budding process could be initiated by the action of PKD on its substrate, phosphatidylinositol 4-kinase IIIb (PI4KIII- $\beta$ ), a known enhancer of vesicle fission. ${ }^{90}$ Vesicles, formed following 
activation of PI4KIII- $\beta$, predominantly move to the cell surface. Thus, PKD could be essential for GLUT4 (and CD36) vesicle translocation to the plasma membrane. Interestingly, it has been shown that PKD is involved in GLUT4-mediated glucose uptake in contracting cardiomyocytes. ${ }^{55}$ Following vesicle budding, and depending on its protein cargo, these vesicles are coated with specific adaptor proteins. Once coated, vesicle destination depends on the docking protein. Using yeast two-hybrid screening, VAMP was identified as a novel binding partner of PKD. ${ }^{91}$ It has also been determined that PKD directly interacts with VAMP, and that this complex regulates vesicular movement for cell surface docking. ${ }^{91}$

\section{Aim of thesis}

Changes in cardiac metabolism, contractility, and cardiac remodeling all have been described to contribute to cardiomyopathy and eventually heart failure, such as seen in diabetes. PKD has been shown to function in several signaling cascades that direct these processes. In this thesis, we strengthen the link that PKD provides between cardiac metabolism, contractility and hypertrophy. It has been shown that PKD is involved in GLUT4-mediated glucose uptake in contracting cardiomyocytes. In Chapter 3 we describe the pathway by which PKD is activated during contraction to induce specifically glucose uptake but not LCFA uptake. Incidently, during a study into PKD phosphorylation we observed that the p-PKD-Ser744/748 antibody also detected cMyBP-C in contracting cardiomyocytes (Chapter 4). Because cMyBP-C has been shown to be a downstream target of PKD, we investigated how PKD could affect contractility of the heart by phosphorylating cMyBP-C (Chapter 5). A wellstudied inducer of glucose uptake, AMPK, was compared with PKD in the process of cardiomyocyte contraction. The PKD and AMPK signaling pathways were compared for their effect on glucose as well as LCFA uptake in contracting and in insulin resistant cardiomyocytes (Chapter 6). Chapter 7 shows how PKD links cardiac metabolism to cell growth. We describe the downstream mechanism by which PKD connects increased glucose uptake with hypertrophic remodeling in cardiac specific PKD1 overexpression mice (caPKD1) mice. In caPKD1 mice, this cardiac hypertrophy can be controlled by a high fat diet. On the other hand, also obesity-induced cardiac remodeling and insulin resistance could be prevented in caPKD1 mice on a high fat diet. In Chapter $\mathbf{8}$ it will be discussed how PKD integrates with other pathways in an attempt to coordinate cardiac metabolism, contractility and structural remodeling. 


\section{References}

1. Hinken AC, Solaro RJ. A dominant role of cardiac molecular motors in the intrinsic regulation of ventricular ejection and relaxation. Physiology (Bethesda). Apr 2007;22:73-80.

2. Carley AN, Severson DL. What are the biochemical mechanisms responsible for enhanced fatty acid utilization by perfused hearts from type 2 diabetic $\mathrm{db} / \mathrm{db}$ mice? Cardiovasc Drugs Ther. Apr 2008;22(2):83-89.

3. Kagaya $Y$, Kanno $Y$, Takeyama D, et al. Effects of long-term pressure overload on regional myocardial glucose and free fatty acid uptake in rats. A quantitative autoradiographic study. Circulation. Apr 1990;81(4):1353-1361.

4. Jameel MN, Zhang J. Myocardial energetics in left ventricular hypertrophy. Curr Cardiol Rev. Aug 2009;5(3):243-250.

5. Boudina S, Abel ED. Diabetic cardiomyopathy, causes and effects. Rev Endocr Metab Disord. Mar 2010;11(1):31-39.

6. Giedd KN, Bergmann SR. Fatty acid imaging of the heart. Curr Cardiol Rep. Apr 2011;13(2):121131.

7. Glatz JF, Luiken JJ, Bonen A. Membrane fatty acid transporters as regulators of lipid metabolism: implications for metabolic disease. Physiol Rev. Jan 2010;90(1):367-417.

8. Kolwicz SC, Jr., Tian R. Glucose metabolism and cardiac hypertrophy. Cardiovasc Res. May 1 2011;90(2):194-201.

9. Glatz JF, Bonen A, Ouwens DM, et al. Regulation of sarcolemmal transport of substrates in the healthy and diseased heart. Cardiovasc Drugs Ther. Dec 2006;20(6):471-476.

10. Korzick DH. From syncitium to regulated pump: a cardiac muscle cellular update. Adv Physiol Educ. Mar 2011;35(1):22-27.

11. Schaub MC, Hefti MA, Zuellig RA, et al. Modulation of contractility in human cardiac hypertrophy by myosin essential light chain isoforms. Cardiovasc Res. Feb 1998;37(2):381-404.

12. Solaro RJ. 2011.

13. Bardswell SC, Cuello F, Rowland AJ, et al. Distinct sarcomeric substrates are responsible for protein kinase D-mediated regulation of cardiac myofilament $\mathrm{Ca} 2+$ sensitivity and cross-bridge cycling. J Biol Chem. Feb 19 2010;285(8):5674-5682.

14. Sumandea MP, Burkart EM, Kobayashi T, et al. Molecular and integrated biology of thin filament protein phosphorylation in heart muscle. Ann N Y Acad Sci. May 2004;1015:39-52.

15. Hamdani N, de Waard M, Messer AE, et al. Myofilament dysfunction in cardiac disease from mice to men. J Muscle Res Cell Motil. 2008;29(6-8):189-201.

16. Witt CC, Gerull B, Davies MJ, et al. Hypercontractile properties of cardiac muscle fibers in a knock-in mouse model of cardiac myosin-binding protein-C. J Biol Chem. Feb 16 2001;276(7):5353-5359.

17. Schlossarek S, Mearini G, Carrier L. Cardiac myosin-binding protein C in hypertrophic cardiomyopathy: mechanisms and therapeutic opportunities. J Mol Cell Cardiol. Apr 2011;50(4):613-620.

18. Harris SP, Bartley CR, Hacker TA, et al. Hypertrophic cardiomyopathy in cardiac myosin binding protein-C knockout mice. Circ Res. Mar 22 2002;90(5):594-601.

19. James J, Robbins J. Signaling and myosin-binding protein C. J Biol Chem. Mar 25 2011;286(12):9913-9919.

20. van der Vusse GJ, Glatz JF, Stam HC, et al. Fatty acid homeostasis in the normoxic and ischemic heart. Physiol Rev. Oct 1992;72(4):881-940.

21. Klabunde RE. Cardiovascular Physiology Concepts 2004.

22. Taha M, Lopaschuk GD. Alterations in energy metabolism in cardiomyopathies. Ann Med. 2007;39(8):594-607.

23. Luiken JJ, Turcotte LP, Bonen A. Protein-mediated palmitate uptake and expression of fatty acid transport proteins in heart giant vesicles. J Lipid Res. Jun 1999;40(6):1007-1016.

24. Abumrad NA, el-Maghrabi MR, Amri EZ, et al. Cloning of a rat adipocyte membrane protein implicated in binding or transport of long-chain fatty acids that is induced during preadipocyte differentiation. Homology with human CD36. J Biol Chem. Aug 25 1993;268(24):17665-17668. 
25. Chabowski A, Gorski J, Luiken JJ, et al. Evidence for concerted action of FAT/CD36 and FABPpm to increase fatty acid transport across the plasma membrane. Prostaglandins Leukot Essent Fatty Acids. Nov-Dec 2007;77(5-6):345-353.

26. Abel ED. Glucose transport in the heart. Front Biosci. Jan 1 2004;9:201-215.

27. Pessin JE, Bell GI. Mammalian facilitative glucose transporter family: structure and molecular regulation. Annu Rev Physiol. 1992;54:911-930.

28. Mueckler M. Facilitative glucose transporters. Eur J Biochem. Feb 1 1994;219(3):713-725.

29. Luiken JJ, Coort SL, Koonen DP, et al. Regulation of cardiac long-chain fatty acid and glucose uptake by translocation of substrate transporters. Pflugers Arch. Apr 2004;448(1):1-15.

30. Joost HG, Thorens B. The extended GLUT-family of sugar/polyol transport facilitators: nomenclature, sequence characteristics, and potential function of its novel members (review). Mol Membr Biol. Oct-Dec 2001;18(4):247-256.

31. Cheeseman C. GLUT7: a new intestinal facilitated hexose transporter. Am J Physiol Endocrinol Metab. Aug 2008;295(2):E238-241.

32. Kraegen EW, Sowden JA, Halstead MB, et al. Glucose transporters and in vivo glucose uptake in skeletal and cardiac muscle: fasting, insulin stimulation and immunoisolation studies of GLUT1 and GLUT4. Biochem J. Oct 1 1993;295 ( Pt 1):287-293.

33. Fischer $\mathrm{Y}$, Thomas J, Sevilla $\mathrm{L}$, et al. Insulin-induced recruitment of glucose transporter 4 (GLUT4) and GLUT1 in isolated rat cardiac myocytes. Evidence of the existence of different intracellular GLUT4 vesicle populations. J Biol Chem. Mar 14 1997;272(11):7085-7092.

34. Watson RT, Pessin JE. Subcellular compartmentalization and trafficking of the insulinresponsive glucose transporter, GLUT4. Exp Cell Res. Nov 15 2001;271(1):75-83.

35. Nishimura H, Pallardo FV, Seidner GA, et al. Kinetics of GLUT1 and GLUT4 glucose transporters expressed in Xenopus oocytes. J Biol Chem. Apr 25 1993;268(12):8514-8520.

36. Muller H, Deckers K, Eckel J. The fatty acid translocase (FAT)/CD36 and the glucose transporter GLUT4 are localized in different cellular compartments in rat cardiac muscle. Biochem Biophys Res Commun. May 3 2002;293(2):665-669.

37. Schwenk RW, Dirkx E, Coumans WA, et al. Requirement for distinct vesicle-associated membrane proteins in insulin- and AMP-activated protein kinase (AMPK)-induced translocation of GLUT4 and CD36 in cultured cardiomyocytes. Diabetologia. Oct 2010;53(10):2209-2219.

38. Zorzano A, Sevilla L, Camps M, et al. Regulation of glucose transport, and glucose transporters expression and trafficking in the heart: studies in cardiac myocytes. Am J Cardiol. Aug 4 1997;80(3A):65A-76A.

39. Depre C, Vanoverschelde JL, Taegtmeyer H. Glucose for the heart. Circulation. Feb 2 1999;99(4):578-588.

40. Choi K, Kim YB. Molecular mechanism of insulin resistance in obesity and type 2 diabetes. Korean J Intern Med. Jun 2010;25(2):119-129.

41. Van Obberghen E, Baron V, Delahaye L, et al. Surfing the insulin signaling web. Eur J Clin Invest. Nov 2001;31(11):966-977.

42. Stump CS, Henriksen EJ, Wei Y, et al. The metabolic syndrome: role of skeletal muscle metabolism. Ann Med. 2006;38(6):389-402.

43. Cho JY, Park J. Contribution of natural inhibitors to the understanding of the PI3K/PDK1/PKB pathway in the insulin-mediated intracellular signaling cascade. Int J Mol Sci. Nov 2008;9(11):2217-2230.

44. Tremblay $\mathrm{F}$, Lavigne $\mathrm{C}$, Jacques $\mathrm{H}$, et al. Defective insulin-induced GLUT4 translocation in skeletal muscle of high fat-fed rats is associated with alterations in both Akt/protein kinase B and atypical protein kinase C (zeta/lambda) activities. Diabetes. Aug 2001;50(8):1901-1910.

45. Standaert ML, Bandyopadhyay G, Perez L, et al. Insulin activates protein kinases C-zeta and Clambda by an autophosphorylation-dependent mechanism and stimulates their translocation to GLUT4 vesicles and other membrane fractions in rat adipocytes. J Biol Chem. Sep 3 1999;274(36):25308-25316.

46. Sharma N, Arias EB, Sajan MP, et al. Insulin resistance for glucose uptake and Akt2 phosphorylation in the soleus, but not epitrochlearis, muscles of old vs. adult rats. J Appl Physiol. Jun 2010;108(6):1631-1640. 
47. Chen YA, Scheller RH. SNARE-mediated membrane fusion. Nat Rev Mol Cell Biol. Feb 2001;2(2):98-106.

48. Sollner T, Bennett MK, Whiteheart SW, et al. A protein assembly-disassembly pathway in vitro that may correspond to sequential steps of synaptic vesicle docking, activation, and fusion. Cell. Nov 5 1993;75(3):409-418.

49. Bryant NJ, Govers R, James DE. Regulated transport of the glucose transporter GLUT4. Nat Rev Mol Cell Biol. Apr 2002;3(4):267-277.

50. Till M, Kolter T, Eckel J. Molecular mechanisms of contraction-induced translocation of GLUT4 in isolated cardiomyocytes. Am J Cardiol. Aug 4 1997;80(3A):85A-89A.

51. Misra P. AMP activated protein kinase: a next generation target for total metabolic control. Expert Opin Ther Targets. Jan 2008;12(1):91-100.

52. Musi N, Hirshman MF, Arad M, et al. Functional role of AMP-activated protein kinase in the heart during exercise. FEBS Lett. Apr 11 2005;579(10):2045-2050.

53. Mu J, Brozinick JT, Jr., Valladares $\mathrm{O}$, et al. A role for AMP-activated protein kinase in contraction- and hypoxia-regulated glucose transport in skeletal muscle. Mol Cell. May 2001;7(5):1085-1094.

54. Habets DD, Coumans WA, El Hasnaoui M, et al. Crucial role for LKB1 to AMPKalpha2 axis in the regulation of $\mathrm{CD} 36$-mediated long-chain fatty acid uptake into cardiomyocytes. Biochim Biophys Acta. Mar 2009;1791(3):212-219.

55. Luiken JJ, Vertommen D, Coort SL, et al. Identification of protein kinase $D$ as a novel contraction-activated kinase linked to GLUT4-mediated glucose uptake, independent of AMPK. Cell Signal. Mar 2008;20(3):543-556.

56. Wong AK, Howie J, Petrie JR, et al. AMP-activated protein kinase pathway: a potential therapeutic target in cardiometabolic disease. Clin Sci (Lond). Apr 2009;116(8):607-620.

57. Sancho Solis R, Ge Y, Walker JW. A preferred AMPK phosphorylation site adjacent to the inhibitory loop of cardiac and skeletal troponin I. Protein Sci. May 2011;20(5):894-907.

58. Kheifets V, Mochly-Rosen D. Insight into intra- and inter-molecular interactions of PKC: design of specific modulators of kinase function. Pharmacol Res. Jun 2007;55(6):467-476.

59. Wang QJ. PKD at the crossroads of DAG and PKC signaling. Trends Pharmacol Sci. Jun 2006;27(6):317-323.

60. Braiman L, Alt A, Kuroki T, et al. Protein kinase Cdelta mediates insulin-induced glucose transport in primary cultures of rat skeletal muscle. Mol Endocrinol. Dec 1999;13(12):20022012.

61. Luiken JJ, Ouwens DM, Habets DD, et al. Permissive action of protein kinase C-zeta in insulininduced CD36- and GLUT4 translocation in cardiac myocytes. J Endocrinol. May 2009;201(2):199-209.

62. Russ $\mathrm{M}$, Eckel J. Insulin action on cardiac glucose transport: studies on the role of protein kinase C. Biochim Biophys Acta. Feb 16 1995;1265(1):73-78.

63. Endoh M, Fujita $S$, Yang HT, et al. Endothelin: receptor subtypes, signal transduction, regulation of $\mathrm{Ca} 2+$ transients and contractility in rabbit ventricular myocardium. Life Sci. 1998;62(17-18):1485-1489.

64. Luiken JJ, Coort SL, Koonen DP, et al. Signalling components involved in contraction-inducible substrate uptake into cardiac myocytes. Proc Nutr Soc. May 2004;63(2):251-258.

65. Niu W, Bilan PJ, Yu J, et al. PKCepsilon regulates contraction-stimulated GLUT4 traffic in skeletal muscle cells. J Cell Physiol. Jan 2011;226(1):173-180.

66. Molnar A, Borbely A, Czuriga D, et al. Protein kinase C contributes to the maintenance of contractile force in human ventricular cardiomyocytes. J Biol Chem. Jan 9 2009;284(2):10311039.

67. Kooij V, Boontje N, Zaremba R, et al. Protein kinase C alpha and epsilon phosphorylation of troponin and myosin binding protein $\mathrm{C}$ reduce $\mathrm{Ca} 2+$ sensitivity in human myocardium. Basic Res Cardiol. Mar 2010;105(2):289-300.

68. Lindhout DA, Li MX, Schieve D, et al. Effects of T142 phosphorylation and mutation R145G on the interaction of the inhibitory region of human cardiac troponin I with the C-domain of human cardiac troponin C. Biochemistry. Jun 11 2002;41(23):7267-7274. 
69. Burkart EM, Arteaga GM, Sumandea MP, et al. Altered signaling surrounding the C-lobe of cardiac troponin $\mathrm{C}$ in myofilaments containing an alpha-tropomyosin mutation linked to familial hypertrophic cardiomyopathy. J Mol Cell Cardiol. Oct 2003;35(10):1285-1293.

70. Barefield D, Sadayappan S. Phosphorylation and function of cardiac myosin binding protein-C in health and disease. J Mol Cell Cardiol. May 2010;48(5):866-875.

71. Lint JV, Rykx A, Vantus T, et al. Getting to know protein kinase D. Int J Biochem Cell Biol. Jun 2002;34(6):577-581.

72. Storz P, Doppler H, Toker A. Activation loop phosphorylation controls protein kinase Ddependent activation of nuclear factor kappaB. Mol Pharmacol. Oct 2004;66(4):870-879.

73. Phan D, Stratton MS, Khai Huynh Q, et al. A novel protein kinase $C$ target site in protein kinase $\mathrm{D}$ is phosphorylated in response to signals for cardiac hypertrophy. Biochem Biophys Res Commun. Jul 29 2011;411(2):335-341.

74. Rozengurt E, Rey O, Waldron RT. Protein kinase D signaling. J Biol Chem. Apr 8 2005;280(14):13205-13208.

75. Rykx A, De Kimpe L, Mikhalap S, et al. Protein kinase D: a family affair. FEBS Lett. Jul 3 2003;546(1):81-86.

76. Matthews SA, Rozengurt E, Cantrell D. Characterization of serine 916 as an in vivo autophosphorylation site for protein kinase D/Protein kinase Cmu. J Biol Chem. Sep 10 1999;274(37):26543-26549.

77. Carnegie GK, Smith FD, McConnachie G, et al. AKAP-Lbc nucleates a protein kinase D activation scaffold. Mol Cell. Sep 24 2004;15(6):889-899.

78. Manni S, Mauban JH, Ward CW, et al. Phosphorylation of the CAMP-dependent protein kinase (PKA) regulatory subunit modulates PKA-AKAP interaction, substrate phosphorylation, and calcium signaling in cardiac cells. J Biol Chem. Aug 29 2008;283(35):24145-24154.

79. Wong W, Scott JD. AKAP signalling complexes: focal points in space and time. Nat Rev Mol Cell Biol. Dec 2004;5(12):959-970.

80. Storz P. Mitochondrial ROS--radical detoxification, mediated by protein kinase D. Trends Cell Biol. Jan 2007;17(1):13-18.

81. Storz P, Doppler $H$, Johannes FJ, et al. Tyrosine phosphorylation of protein kinase $D$ in the pleckstrin homology domain leads to activation. J Biol Chem. May 16 2003;278(20):1796917976.

82. Itoh $S$, Lemay $S$, Osawa $M$, et al. Mitochondrial Dok-4 recruits Src kinase and regulates NFkappaB activation in endothelial cells. J Biol Chem. Jul 15 2005;280(28):26383-26396.

83. Robles-Flores $M$, Rendon-Huerta $E$, Gonzalez-Aguilar $H$, et al. p32 (gC1qBP) is a general protein kinase C (PKC)-binding protein; interaction and cellular localization of P32-PKC complexes in ray hepatocytes. J Biol Chem. Feb 15 2002;277(7):5247-5255.

84. Storz $P$, Hausser $A$, Link $G$, et al. Protein kinase $C$ [micro] is regulated by the multifunctional chaperon protein p32. J Biol Chem. Aug 11 2000;275(32):24601-24607.

85. Waldron RT, Whitelegge JP, Faull KF, et al. Identification of a novel phosphorylation site in cjun directly targeted in vitro by protein kinase D. Biochem Biophys Res Commun. May 4 2007;356(2):361-367.

86. Wang Y, Waldron RT, Dhaka A, et al. The RAS effector RIN1 directly competes with RAF and is regulated by 14-3-3 proteins. Mol Cell Biol. Feb 2002;22(3):916-926.

87. Fielitz J, Kim MS, Shelton JM, et al. Requirement of protein kinase D1 for pathological cardiac remodeling. Proc Natl Acad Sci U S A. Feb 26 2008;105(8):3059-3063.

88. Mora S, Pessin JE. The MEF2A isoform is required for striated muscle-specific expression of the insulin-responsive GLUT4 glucose transporter. J Biol Chem. May 26 2000;275(21):1632316328.

89. Malhotra V, Campelo F. PKD regulates membrane fission to generate TGN to cell surface transport carriers. Cold Spring Harb Perspect Biol. Feb 2011;3(2).

90. Graham TR, Burd CG. Coordination of Golgi functions by phosphatidylinositol 4-kinases. Trends Cell Biol. Feb 2011;21(2):113-121.

91. Lu G, Chen J, Espinoza LA, et al. Protein kinase D 3 is localized in vesicular structures and interacts with vesicle-associated membrane protein 2. Cell Signal. Apr 2007;19(4):867-879. 


\section{CHAPTER 2}

\section{High fat diet induced diabetic cardiomyopathy}

Ellen Dirkx, Robert W. Schwenk, Jan F.C. Glatz, Joost J.F.P. Luiken, Guillaume J.J.M. van Eys

Department of Molecular Genetics, Cardiovascular Research Institute Maastricht (CARIM), Maastricht University, the Netherlands 


\begin{abstract}
In response to a chronic high plasma concentration of long-chain fatty acids (FAs), the heart is forced to increase the uptake of FA at the cost of glucose. This switch in metabolic substrate uptake is accompanied by an increased presence of the FA transporter CD36 at the cardiac plasma membrane and over time results in the development of cardiac insulin resistance and ultimately diabetic cardiomyopathy. FA interact with peroxisome proliferator-activated receptors (PPARs), which induce upregulation of the expression of enzymes necessary for their disposal through mitochondrial $\beta$-oxidation, but also of proteins that stimulate FA uptake. This then leads to a further increase in intracellular FA concentration in cardiomyocytes. These metabolic changes are supposed to play an important role in the development of cardiomyopathy. While the onset of this pathology is an increased FA utilization by the heart, the subsequent lipid overload results in an increased production of reactive oxygen species (ROS) and accumulation of lipid intermediates such as diacylglycerol (DAG) and ceramides. These compounds have a profound impact on signaling pathways, in particular insulin signaling. Over time the metabolic changes will introduce structural changes that affect cardiac contractile characteristics. The present mini-review focuses on the lipid-induced changes that link metabolic perturbation, characteristic for type 2 diabetes, with cardiac remodeling and dysfunction.
\end{abstract}




\section{Introduction}

Myocardial metabolic remodeling is central to the pathogenesis of cardiac diseases such as ventricular hypertrophy and diabetic cardiomyopathy. To produce energy, the heart utilizes mainly long-chain fatty acids (FA) and glucose, and minor quantities of lactate and ketone bodies. ${ }^{1}$ Under normal conditions, the majority of the acetyl-CoA that enters the Krebs cycle is generated by $\beta$-oxidation of FA, while about a third is derived from oxidation of pyruvate, which is the product of glycolysis. ${ }^{2}$ The shift in oxidation towards FA, at the cost of glucose, that occurs in type 2 diabetes is well documented. ${ }^{3}$ Less studied is the mechanism by which diabetes affects morphology and structure of the heart. However, there are a number of reports that link type 2 diabetes to a disproportionate increase in left ventricular wall mass. ${ }^{4,5}$ At present, metabolic disturbances due to lipid overload are thought to be the underlying cause of cardiac hypertrophy in type 2 diabetes.

\section{Fatty acid uptake and hyperlypidemia}

In the heart FAs are substrate for $\beta$-oxidation, and they cover $60-70 \%$ of the energy needed for the generation of ATP. Since the heart must respond to continuously changing energy demands but has only a limited capacity for storage of either FA or glucose, substrate uptake must match energy demands. FA can be taken up by passive diffusion (20\%) however most transport is protein-mediated (80\%). ${ }^{6}$ In cardiac myocytes this protein-mediated transport of FA depends largely on the plasma membrane transporter CD36 and the plasmalemmal fatty acid-binding protein (FABPpm). ${ }^{7}$

The FA transporter CD36 is a 472-amino acid ( $88 \mathrm{kDa}$ ) protein that has a hairpin membrane topology with two transmembrane spanning regions, with both the $\mathrm{NH}_{2}$ and $\mathrm{COOH}$ termini as short segments in the cytoplasm. ${ }^{8}$ The current knowledge on its structure has been extensively reviewed by Glatz et al. ${ }^{9}$ In cardiac myocytes of CD36 knockout (CD36KO) mice, insulin stimulation of FA uptake was markedly impaired $(+21 \%)$ compared with wild type mice $(+60 \%) .{ }^{10}$ These reductions in FA uptake also contributed to altered rates of FA metabolism. For example, in working hearts, FA oxidation was 40-60\% lower in CD36KO than in WT mice. ${ }^{11,12}$ In both heart and skeletal muscle FA uptake rates have been found to run in parallel with the expression level of $\mathrm{CD} 36 .^{9}$ Thus, $\mathrm{CD} 36$ overexpression results in elevated rates of FA uptake ${ }^{13}$ whereas CD36KO or knockdown impairs the transport of FA across the plasma membrane. ${ }^{14}$ It is remarkable that Asian and African individuals relatively frequently have a genetic CD36 deficiency. CD36 deficiency showed some features of the 'metabolic syndrome'. Middle aged patients had significantly higher plasma triacylglycerol concentrations, lower high-density lipoprotein cholesterol levels, higher plasma glucose levels, and higher blood pressure than aged-matched control subjects. The cardiovascular pathophysiology of these individuals underscores the importance of CD36 for transport of FA into cardiomyocytes. ${ }^{15}$ 
During increased contraction or insulin stimulation, CD36 translocates from endosomal pools to the plasma membrane to mediate FA uptake. ${ }^{16}$ In the cytoplasm FA bind to cytosolic fatty acid-binding protein $\left(F A B P_{c}\right)$ after which they are transported through the cytoplasm to the mitochondria. Then, fatty acyl-CoA synthetase (ACS) present at the outer mitochondrial membrane esterifies FA into acyl-CoA (Fig. 1) ${ }^{17}$, after which this metabolite is converted into acyl-carnitine by carnitine palmitoyl transferase I (CPT-I) ${ }^{18,19}$ and translocated across the inner mitochondrial membrane by carnitine acyl translocase (CAT). Inside the mitochondrial matrix, carnitine palmitoyl transferase II (CPT-II) regenerates acyl-CoA, which then undergoes boxidation (Fig. 1). ${ }^{18,19}$ These transferase reactions are rate-limiting steps in oxidation. Furthermore, FA oxidation rates are directly dependent on myocardial substrate availability, which is regulated primarily by levels of malonyl-CoA, a physiological inhibitor of CPT-I. ${ }^{20}$

Fig. 1

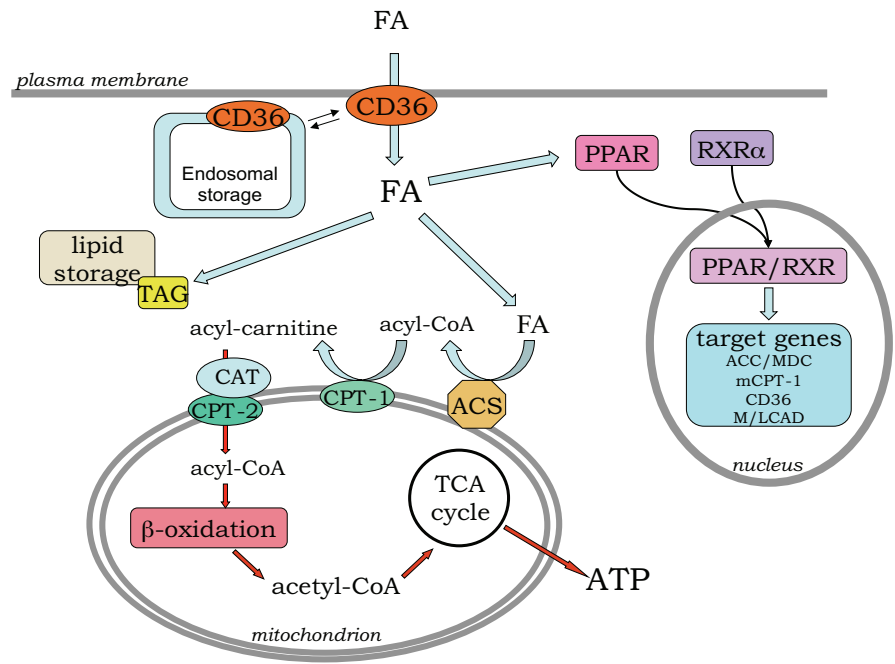

Fig.1 Regulation of long-chain fatty acid (FA) uptake and oxidation in cardiomyocytes. FAs are imported from the blood facilitated by CD36 or by passive diffusion. FAs then are activated by ACS to acyl-CoA. Acyl-CoA is converted into acyl-carnitine by carnitine palmitoyl transferase I (CPT-1). Acyl-carnitine is translocated into the mitochondria by carnitine acyl translocase (CAT). In the mitochondrial matrix, CPT-II regenerates acyl-CoA, which enters $\beta$-oxidation and is further processed to ATP. Besides oxidation, cytoplasmic FA can be stored or can interact with peroxisome proliferator-activated receptors (PPARs) to stimulate the expression of genes coding for lipid metabolic enzymes and transporters.

\section{Hyperlipidemia and diabetes}

The diabetic state is characterized by hyperinsulinemia, late-onset hyperglycemia and hyperlipidemia; the latter as a result of increased plasma levels of triacylglyceroles and (non-esterified) FA. ${ }^{21}$ In advanced diabetes, the combined effects of high levels of circulating FA and of insulin resistance drive cardiomyocytes towards an almost exclusive use of FA to generate ATP. 
Diabetic cardiomyopathy is associated with an increase in FA uptake and oxidation, and an increase in cytoplasmic FA concentration. ${ }^{22}$ Cardiomyocytes respond to increased FA concentration by upregulating the expression of the enzymes necessary for their utilization through mitochondrial $\beta$-oxidation. ${ }^{23}$ These enzymes are under transcriptional control of PPAR $\alpha$ and PPAR $\beta / \delta$ (see below). In addition, diabetic FA levels inhibit pyruvate dehydrogenase, which impairs myocardial energy production and leads to accumulation of both glycolytic intermediates and intracellular lipids, because the demand for FA oxidation then exceeds the mitochondrial capacity. $^{24}$

\section{The role of PPARs}

Cardiac FA utilization is largely controlled by metabolic gene programs that are under the control of nuclear receptors that bind FA or its derivatives. ${ }^{25,}{ }^{26}$ Peroxi- $^{2}$ some proliferator-activated receptors (PPARs) are members of a nuclear receptor superfamily and some of them function as FA sensors and as transcriptional regulators of FA uptake and oxidation. ${ }^{27}$ Upon activation PPARs form heterodimers with retinoid $X$ receptors (RXR) and then bind to so-called PPAR responsive elements (PPREs) located in $5^{\prime}$ upstream regions of a number of genes encoding metabolic enzymes (Fig 1). ${ }^{28}$ The binding to PPREs is enhanced by peroxisome proliferatoractivated receptor gamma coactivator-1 (PGC-1) Once bound to the PPRE, the PPAR/RXR/PGC-1 complex increases the rate of transcription of, amongst others, genes involved in FA transport and oxidation. ${ }^{29}$ For instance, PPAR $\alpha$ upregulates the expression of FA transporters such as fatty acid-transport protein (FATP). CD36, however, has no PPRE in the upstream promoter region, but still is activated by PPAR ligands, probably in an indirect manner. ${ }^{30}$ PPAR $\alpha$ also induces the synthesis of metabolic enzymes of the $\beta$-oxidation pathway, such as CPT-I and acyl-CoA dehydrogenase. ${ }^{23,31,32}$ In the promoter region of each of these latter proteins functional PPREs have been identified. While under standard conditions PPAR $\alpha$ stimulates FA oxidation, under diabetic conditions PPAR $\alpha$ agonists, such as fibrates, downregulate FA oxidation and upregulate glucose oxidation. Such a shift towards glucose utilization may be due to normalization of circulating triacylglyceroles and FA concentrations, as observed in $d b / d b$ mice and in diabetic Zucker rats. ${ }^{33,34}$ The importance of the whole body effects seems to be underscored by results in transgenic mice with cardiac specific PPAR $\alpha$ overexpression. In the hearts of these mice, an increase of FA and a decrease of glucose utilization was found, ${ }^{35}$ an effect that was exacerbated by a high fat diet. ${ }^{36}$ In line with this, PPAR $\alpha$ KO display no severe phenotypic defects. However, the hearts of these mice exhibit serious metabolic abnormalities. When challenged by a high cytoplasmic FA concentration, these mice are unable to up-regulate cardiac transporters and enzymes crucial for FA metabolism. The mice accumulate myocardial lipids, due to a lower FA oxidation rate but they are resistant to high fat-induced insulin resistance. ${ }^{37,38}$ Interestingly, the absence of PPAR $\alpha$ was found to result in a decreased expression of malonyl-CoA carboxylase, leading to increased malonyl-CoA concentrations which strongly inhibit FA oxida- 
tion. ${ }^{39}$ The complex picture that arises from the available data establishes a role for PPAR $\alpha$ in cardiac metabolic homeostasis, but at the same time demands further study. Such studies have to include the other PPAR types, in particular PPAR $\beta / \delta$, since these are also highly expressed in cardiac myocytes and increasing evidence points towards a significant role of other PPAR types in myocardial lipid homeostasis. $^{40}$

\section{CD36 relocation}

Exposure to a high fat diet induces cardiac contractile dysfunction, which is associated with a permanent relocation of CD36 to the plasma membrane. ${ }^{41}$ Furthermore, relocation of CD36 appears to be a general phenomenon in insulin resistant hearts, ${ }^{42}$ and precedes cardiac contractile dysfunction. ${ }^{41}$ Also, in skeletal muscle biopsies from type 2 diabetic subjects, CD36 was relocated to the sarcolemma, and this increased sarcolemmal localization of CD36 is closely correlated with muscular triacylglycerol accumulation. ${ }^{43}$

This raises the question what mechanism underlies the continuous presence of CD36 at the cardiac plasma membrane in case of a high fat diet. Cardiac CD36 is stored in endosomal storage pools that are regulated by AMP-activated kinase (AMPK) and PI3-kinase (PI3K)-Akt, respectively. Activation of AMPK is critical for contraction induced CD36 translocation to the plasma membrane, and activation of PI3K-Akt is critical for insulin-induced CD36 translocation (reviewed in ${ }^{9}$ ). In rats on a high fat diet, cardiac basal Akt phosphorylation was elevated, whereas insulinstimulated Akt phosphorylation, CD36 translocation, and FA uptake each were decreased. ${ }^{41}$ However, AMPK activity was not affected by this diet. These observations suggest that an increased basal Akt activity may contribute to the continuous presence of CD36 at the plasma membrane in hearts during high fat diet feeding (Fig 2).

Another potential mechanism to stimulate relocation of CD36 to the plasma membrane is via PPAR $\alpha$ and PPAR $\beta / \delta$ activation (Fig 2). It has been shown that these PPARs are able to upregulate FA uptake and oxidation in the heart by inducing the translocation of CD36 to the plasma membrane. Importantly, observed effects of continuous PPAR activation were not related to AMPK activation or plasma FA concentrations. ${ }^{44}$ Furthermore, oral treatment with the PPAR 8 agonist GW501516 was associated with large increases in total muscle CD36 protein content, but not diacylglycerol or ceramide contents. ${ }^{45}$ Taken together, this indicates that PPAR activation can induce both CD36 expression and its relocation to the plasma membrane. 
Fig. 2

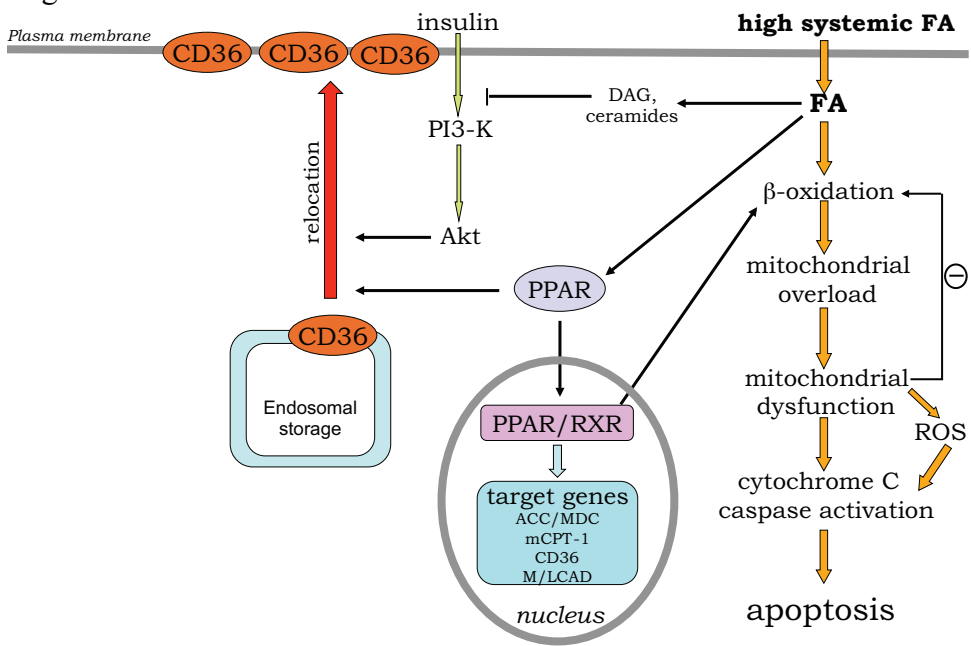

Fig.2 Schematic depiction of consequences of increased FA uptake by cardiomyocytes (metabolic remodeling). High plasma FA concentrations lead to an increase in cytoplasmic FA levels. This stimulates $\beta$ oxidation (directly or indirectly via PPAR) and also stimulates CD36 relocation to the plasma membrane (via PPAR or via increased basal Akt phosphorylation), which leads to an even further increase in cellular FA uptake. The increased intracellular FA concentration causes mitochondrial overload, leading to dysfunction and oxygen radical production. Eventually, this results in cytochrome $c$ leakage and in caspase activation, which induces apoptosis.

\section{Insulin signaling and hyperlipidemia}

Insulin-stimulated glucose and FA uptake are initiated by binding of insulin to the $\alpha$ subunit of the insulin receptor at the sarcolemma. ${ }^{46}$ This induces a conformational change resulting in the autophosphorylation of a number of tyrosine residues present in the $\beta$-subunits. ${ }^{47}$ Tyrosine autophosphorylation leads to activation of the receptor's intrinsic tyrosine kinase activity, which in turn results in phosphorylation of the two insulin receptor substrates IRS-1 and IRS-2. Phosphorylated IRS1/2 then binds and activates the regulatory subunit of phosphotidylinositol 3-kinase (PI3K) to produce phosphatidylinositol 3,4,5-triphosphate, and activate phosphoinositidedependent protein kinases, kinase B/Akt, 3-phosphoinositide-dependent kinase-1 (PDK1), as well as the atypical protein kinase $C$ isoforms $\zeta$ and $\lambda .^{46,4849}$ PDK1 and PKC $\zeta / \lambda$ are necessary for both GLUT4 ${ }^{50,51}$ and CD36 ${ }^{52}$ translocation, likely via activation of vesicle-activated membrane protein-2 (VAMP2), to ensure that both transporters are specifically delivered to the sarcolemma, and not to other subcellular membrane systems. ${ }^{53}$ Additionally, activated Akt phosphorylates and inhibits its 160 kDa substrate (AS160), thereby inducing GLUT4 translocation from the intracellular pools to the sarcolemma. ${ }^{50}$ In addition to the effect of insulin on GLUT4, insulin receptor signaling also affects CD36-mediated FA uptake. ${ }^{54,55}$ Whether AS160 also plays a role in insulin-stimulated CD36 translocation has not yet been investigated. 
However, pharmacological experiments and studies in Akt2KO mice suggest that in the heart the CD36 translocation pathway largely parallels the regulation of GLUT4 translocation. ${ }^{9}$

The role of several proteins of the insulin cascade have been tested in transgenic mouse models. Cardiomyocyte-specific insulin receptor knockout mice (CIRKO) displayed decreased glucose and FA oxidation and mitochondrial dysfunction. ${ }^{56}$ Deletion of the Akt2 gene resulted in insulin resistance and type 2 diabetes. ${ }^{57}$ Finally, conditional mouse models have been generated that resulted in an up- or downregulation of PI3K and its downstream effector PDK1, linking the metabolic state to changes in heart morphology. ${ }^{58,59}$ These mouse models confirm the complexity and intrinsic fine-tuning of the insulin-initiated pathway that leads to cardiac substrate uptake. However, the precise nature of the crosstalk between the pathways directing glucose and FA uptake still has to be elucidated.

\section{Insulin resistance}

Insulin resistance is a risk factor of left ventricular dysfunction and heart failure, ${ }^{60}$ and is one of the hallmarks of type 2 diabetes. ${ }^{61,62}$ It is associated with hyperinsulinemia and hyperglycemia. However, despite whole body hyperinsulinemia and hyperglycemia, the diabetic heart relies almost entirely on FA utilization at the expense of glucose. ${ }^{3}$ This change in substrate utilization has been described in rodent models as well as in humans and can be largely attributed to nutrition of excessive fat containing food. Rodents can be made diabetic by prolonged periods of exposure to a high fat diet. Alterations in FA metabolism, that occur long before the diabetic state has been reached, will lead to a permanent relocation of CD36 to the plasma membrane and a reduced insulin-stimulated GLUT4-mediated glucose uptake (see above). ${ }^{63}$ Furthermore, the increase in cytoplasmic FA results in an increased ROS production (as a by-product of increased FA oxidation) and accumulation of lipid intermediates such as diacylglycerol (DAG) and ceramide (as a result of a mismatch between FA uptake and FA oxidation). These latter compounds have a profound impact on insulin signaling. ${ }^{64}$ Kinases, notably JNK and IKK, will be activated by ROS, and PKC will be activated by DAG. Together they will down-regulate insulin action through serine phosphorylation of IRS-1. ${ }^{65}$ In contrast to tyrosine phosphorylation, serine phosphorylation leads to the inhibition of IRS-1. Furthermore, ceramides neutralize insulin action by inhibiting Akt. ${ }^{66}$ Thus, in the diabetic heart intracellular accumulation of lipid metabolites, most notably ceramides and DAG, and generation of ROS promote a permanent CD36 relocation and a decreased insulin-stimulated GLUT4 translocation. The latter decreases the ability of the heart to utilize glucose. 


\section{Diabetic cardiac remodeling}

In a healthy individual, cardiac hypertrophy is the answer to an increased work load. It is a beneficial compensatory process as it decreases wall stress and increases cardiac output and stroke volume. However, hypertrophic growth in the context of disease is in the end maladaptive because it will progress to decompensation, contractile dysfunction and ultimately heart failure. ${ }^{67}$ Physiologically and biochemically, hypertrophic remodeling is a delicate process, which balances between adaptation and disease, and includes participation of a number of pathways that direct growth and differentiation. When challenged, as in diabetes, only a small percentage of the cells in the heart (myoblasts) can divide and proliferate. A recent paper by Efe et al. ${ }^{68}$ describes genetic reprogramming of cardiac fibroblasts into cardiomyocytes. This opens new avenues for cardiac remodeling. Still, cardiac enlargement and capacity increase occurs primarily as a result of growth of resident myocytes. ${ }^{69}$ Myocyte enlargement may follow two patterns: on the one hand concentric hypertrophy is achieved by addition of sarcomeres in parallel, increasing myocyte width; on the other hand eccentric hypertrophy is the result of the addition of sarcomere series, causing the myocyte to elongate. ${ }^{70}$

The cause of concentric hypertrophy is increased stress on the ventricle walls, which adversely affects cardiac output. The increased pressure is balanced by myocyte hypertrophy and by increased wall thickness, which together eventually equalize the wall stress. ${ }^{71}$ This results in a concentric form of hypertrophy in which the ventricle walls increase in thickness while the chamber volume is unaffected. The increase in wall thickness is proportional to the increase in systolic pressure, and wall stress is normalized. However, there is a greater oxygen demand of the heart due to the increased cardiac mass. In eccentric hypertrophy, chamber volume is increased with little or no effect on wall thickness. Ventricular dilation allows for increased pressure necessary for ejection, but wall stress is not normalized. Interestingly, prolonged aortic stenosis also leads to a drop in cardiac output, elevated enddiastolic pressure and dilation of the left ventricle. This suggests that concentric hypertrophy may in time deteriorate into a dilated eccentric growth. ${ }^{72}$

\section{The role of FA}

Diabetic cardiomyopathy is characterized by ventricular dysfunction occurring independently of a recognized cause such as coronary atherosclerosis or hypertension. ${ }^{73}$ Several studies have shown that diabetes results in structural, mostly hypertrophic, and functional cardiac changes and subsequently heart failure. ${ }^{4,74,75}$ The underlying pathological mechanisms of diabetic cardiomyopathy are still poorly understood, although there is accumulating evidence that this cardiomyopathy is associated with an altered metabolism. Increased FA uptake and lipid accumulation in cardiomyocytes are associated with insulin resistance, type 2 diabetes, hypertrophy and eventually heart failure. ${ }^{3,76}$ In this respect, several rodent models have been studied, including rats on a high fat diet, ${ }^{41}$ leptin-deficient animals, ${ }^{77}$ and cardiac PPAR $\alpha$ 
overexpression mice. ${ }^{35}$ Interestingly, the absence of CD36 in cardiac PPAR $\alpha$ overexpression mice prevented myocyte triacylglycerol accumulation and cardiac dysfunction both under basal conditions and following administration of a high fat diet. The rescue of the cardiac PPAR $\alpha$ overexpression phenotype by CD36 ablation was associated with increased glucose uptake and oxidation rather than changes in FA utilization. ${ }^{78}$ All these data indicate that intramyocardial lipid accumulation underlies diabetic cardiac dysfunction. In this respect a recent paper by Glenn et al. ${ }^{79}$ is of interest. These investigators generated a transgenic mouse model with cardiomyocyte-specific overexpression of diacylglycerol acyl-transferase-1 (DGAT1). DGAT1 is involved in synthesizing triacylglyceroles. As expected, the DGAT1 transgenic mice displayed cardiac lipid accumulation. In addition, the mice also exhibited cardiac fibrosis and contractile dysfunction. As a result, this report strongly supports the concept that lipid accumulation in the heart may be directly responsible for metabolic cardiomyopathy.

\section{The role of Akt signaling}

Akt signaling is an important regulator of cardiac growth, and its overexpression leads to enhanced contractility, cell survival and pathological cardiac hypertrophy. ${ }^{80}$, ${ }^{81}$ High fat diet feeding did not change the cardiac expression of Akt and Foxo3a but basal phosphorylation levels of Akt were increased, which leads to inhibition of Foxo3a. ${ }^{82}$ In these hearts, insulin-stimulated phosphorylation of Akt and Foxo3a was blunted, leading to reduced insulin sensitivity. These data also favor a role for Akt and its downstream signal Foxo3a in cardiac hypertrophy during lipid overload (Fig 3). Specifically, increased basal phosphorylation of Akt and decreased activation of Foxo3a, as occurring after high fat diet feeding, promote cardiac hypertrophy and suppress atrophy-specific gene transcription involving atrogin-1 and MuRF-1. ${ }^{82}$ In addition, a reduced insulin action hampers Foxo3a phosphorylation and apoptosis, which was supported by elevated caspase activities in cardiomyocytes after high fat diet intake ${ }^{82}$. The observation of enhanced basal Foxo3a phosphorylation and suppressed atrophy-specific gene suppression coincides with cardiac hypertrophy during lipid overload. Taken together, during a high fat diet, basal Akt phosphorylation levels increase and this will cause both metabolic and hypertrophic cardiac remodeling.

\section{The role of ROS}

In diabetic patients, as in several animal models, ventricular wall remodeling is often compromised by the development of fibrosis, changes in the extracellular matrix and even apoptosis. ${ }^{83-85}$ Such changes are the result of a genetic reprogramming often started by oxidative stress. Excessive FA oxidation in diabetic cardiomyocytes will undoubtedly lead to increased ROS production (Fig 3). As second messenger, ROS can mediate hypertrophic signals by regulating various intracellular signal transduction cascades and the activity of various transcription factors, such as NF-KB and activator protein- $1,{ }^{86}$ and by activating mitogen-activated protein kinases 
(MAPKs). ${ }^{87,88}$ In addition, ROS has been shown to activate matrix metalloproteinases, in particular MMP-2. ${ }^{89}$ MMPs are well known for their role in extracellular matrix remodeling, but they can also cleave sarcomeric proteins such as troponin-I and MLC-1, which leads to contractile dysfunction and may contribute to apoptosis. ${ }^{90}$ Both intracellular and extracellular matrix actions of MMP may affect structure and function of the heart. The standing body of literature on the ROS-MMPhypertrophy axis in ischemia-reperfusion is rather extensive, but in insulin resistance/diabetes this aspect has been neglected so far, since focus has been aimed at the contractile performance.

Although mitochondria are a major source of ROS, the organelles themselves can be damaged by ROS. Mitochondrial injury is reflected by mtDNA damage as well as by a decline in the mtRNA transcripts, protein synthesis and mitochondrial function. ${ }^{91}$ Eventually, this can lead to apoptosis due to the release of proapoptotic proteins by the mitochondria. ${ }^{92}$ In the diabetic heart, mitochondrial oxidative stress induces apoptosis by release of cytochrome $c$ and upregulation of caspase- 3 and of caspase- $9 .{ }^{93,94}$ Thus, in earlier stages of insulin resistance, mitochondrial FA oxidation increases, while in advanced stages of insulin resistance, ROS production leads to mitochondrial injury, which results in a decreased FA oxidation.

Fig. 3

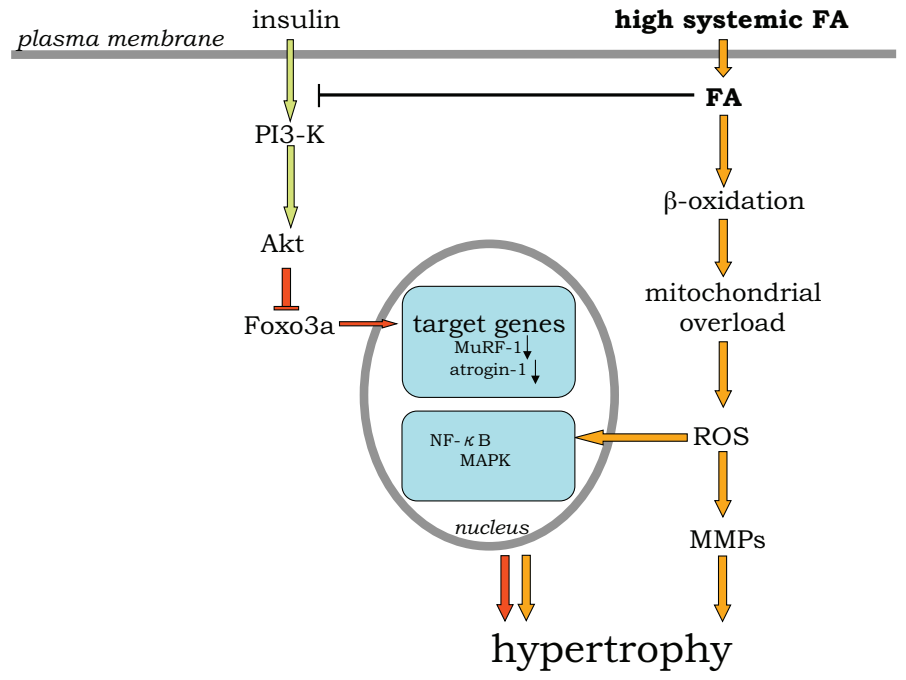

Fig.3 Schematic depiction of the consequences of increased FA uptake by cardiomyocytes (hypertrophic remodeling). Increased cytoplasmic FA concentrations will induce hypertrophic remodeling by at least two mechanisms. Firstly, high cytoplasmic FA levels increase basal Akt phosphorylation, which leads to the inactivation of Foxo3a. Inactivation of Foxo3a leads to the inhibition of several atrophy-related genes, which leads to hypertrophic remodeling. Secondly, high cytoplasmic FA levels cause an increase in mitochondrial ROS generation. This will activate MMPs, which induce extracellular matrix remodeling and cleave sarcomeric proteins leading to cardiac dysfunction and remodeling. In addition, ROS will (indirectly) affect the expression of genes involved in inflammation and growth. 


\section{Concluding remarks}

Our current understanding of the mechanism by which systemic hyperlipidemia leads to cardiac remodeling and dysfunction can be summarized as follows. Initially, high fat diet-induced hyperlipidemia will lead to an increased facilitated diffusion of FA over the plasma membrane into the cytoplasm. The higher intracellular concentration of FA will lead to an activation of PPAR signaling pathways. This not only enhances mitochondrial $\beta$-oxidation but also CD36 translocation, which will speedup FA import and further boost PPAR stimulation. The resulting vicious circle of increased uptake of FA and FA-induced uptake stimulation eventually leads to mitochondrial FA overload. FA becomes the preferred substrate for mitochondrial $\beta$ oxidation at the expense of glucose. Excessive $\beta$-oxidation results in massive ROS production. Over time this may contribute to cardiac remodeling by inducing the expression of genes such as MMP-2. ROS production also can cause mitochondrial dysfunction, which leads to cardiomyocyte apoptosis. Finally, FA overload affects the complex signaling patterns of insulin action and glucose utilization directly (via Akt), and indirectly (via ROS). Thus, whole body lipid overload causes an increased uptake of FA into the heart, facilitated by $\mathrm{CD} 36$, which subsequently hampers glucose oxidation. In the end this is detrimental to the cardiomyocyte. Although the regulation of FA uptake and the effects of FA on ongoing cellular processes are not yet completely understood, the increasing insight in this complex diabetic pathology may already offer opportunities for therapeutic intervention.

\section{Conflict of interest}

The authors declare that there are no conflicts of interest.

\section{Acknowledgements}

Financial support for this work was obtained from the transnational University Limburg. Part of this research was performed within the framework of CTMM, the Center for Translational Molecular Medicine, project PREDICCt (grant 01C-104), and supported by the Netherlands Heart Foundation, Dutch Diabetes Research Foundation, and Dutch Kidney Foundation. 


\section{References}

1. Wentz $A E$, d'Avignon DA, Weber ML, et al. Adaptation of myocardial substrate metabolism to a ketogenic nutrient environment. J Biol Chem. Aug 6 2010;285(32):24447-24456.

2. Scolletta S, Biagioli B. Energetic myocardial metabolism and oxidative stress: let's make them our friends in the fight against heart failure. Biomed Pharmacother. Mar 2010;64(3):203-207.

3. Carley AN, Severson DL. Fatty acid metabolism is enhanced in type 2 diabetic hearts. Biochim Biophys Acta. May 15 2005;1734(2):112-126.

4. Devereux RB, Roman MJ, Paranicas $M$, et al. Impact of diabetes on cardiac structure and function: the strong heart study. Circulation. May 16 2000;101(19):2271-2276.

5. Galderisi M, Anderson KM, Wilson PW, et al. Echocardiographic evidence for the existence of a distinct diabetic cardiomyopathy (the Framingham Heart Study). Am J Cardiol. Jul 1 1991;68(1):85-89.

6. Taha M, Lopaschuk GD. Alterations in energy metabolism in cardiomyopathies. Ann Med. 2007;39(8):594-607.

7. Luiken JJ, Turcotte LP, Bonen A. Protein-mediated palmitate uptake and expression of fatty acid transport proteins in heart giant vesicles. J Lipid Res. Jun 1999;40(6):1007-1016.

8. Tao N, Wagner SJ, Lublin DM. CD36 is palmitoylated on both N- and C-terminal cytoplasmic tails. J Biol Chem. Sep 13 1996;271(37):22315-22320.

9. Glatz JF, Luiken JJ, Bonen A. Membrane fatty acid transporters as regulators of lipid metabolism: implications for metabolic disease. Physiol Rev. Jan 2010;90(1):367-417.

10. Bonen A, Han XX, Habets DD, et al. A null mutation in skeletal muscle FAT/CD36 reveals its essential role in insulin- and AICAR-stimulated fatty acid metabolism. Am J Physiol Endocrinol Metab. Jun 2007;292(6):E1740-1749.

11. Koonen DP, Febbraio M, Bonnet S, et al. CD36 expression contributes to age-induced cardiomyopathy in mice. Circulation. Nov 6 2007;116(19):2139-2147.

12. Kuang $M$, Febbraio $M$, Wagg $C$, et al. Fatty acid translocase/CD36 deficiency does not energetically or functionally compromise hearts before or after ischemia. Circulation. Mar 30 2004;109(12):1550-1557.

13. Garcia-Martinez C, Marotta M, Moore-Carrasco R, et al. Impact on fatty acid metabolism and differential localization of FATP1 and FAT/CD36 proteins delivered in cultured human muscle cells. Am J Physiol Cell Physiol. Jun 2005;288(6):C1264-1272.

14. Bell JA, Reed MA, Consitt LA, et al. Lipid partitioning, incomplete fatty acid oxidation, and insulin signal transduction in primary human muscle cells: effects of severe obesity, fatty acid incubation, and fatty acid translocase/CD36 overexpression. J Clin Endocrinol Metab. Jul 2010;95(7):3400-3410.

15. Hirano K, Kuwasako T, Nakagawa-Toyama $\mathrm{Y}$, et al. Pathophysiology of human genetic CD36 deficiency. Trends Cardiovasc Med. May 2003;13(4):136-141.

16. Schwenk RW, Dirkx E, Coumans WA, et al. Requirement for distinct vesicle-associated membrane proteins in insulin- and AMP-activated protein kinase (AMPK)-induced translocation of GLUT4 and CD36 in cultured cardiomyocytes. Diabetologia. Oct 2010;53(10):2209-2219.

17. Normann PT, Norseth J, Flatmark T. Acyl-CoA synthetase activity of rat heart mitochondria. Substrate specificity with special reference to very-long-chain and isomeric fatty acids. Biochim Biophys Acta. Aug 1 1983;752(3):474-481.

18. Woldegiorgis $\mathrm{G}$, Shi J, Zhu H, et al. Functional characterization of mammalian mitochondrial carnitine palmitoyltransferases I and II expressed in the yeast Pichia pastoris. I Nutr. Feb 2000;130(2S Suppl):310S-314S.

19. de Vries Y, Arvidson DN, Waterham HR, et al. Functional characterization of mitochondrial carnitine palmitoyltransferases I and II expressed in the yeast Pichia pastoris. Biochemistry. Apr 29 1997;36(17):5285-5292.

20. Kudo N, Barr AJ, Barr RL, et al. High rates of fatty acid oxidation during reperfusion of ischemic hearts are associated with a decrease in malonyl-CoA levels due to an increase in 5'-AMPactivated protein kinase inhibition of acetyl-CoA carboxylase. J Biol Chem. Jul 21 1995;270(29):17513-17520.

21. Sato Y, Okajima F, Oikawa S. [Therapeutic points and meaning of dyslipidemia in diabetic patients]. Nippon Rinsho. May 2010;68(5):905-910. 
22. Boudina S, Abel ED. Diabetic cardiomyopathy revisited. Circulation. Jun 26 2007;115(25):32133223.

23. Banke NH, Wende AR, Leone TC, et al. Preferential oxidation of triacylglyceride-derived fatty acids in heart is augmented by the nuclear receptor PPARalpha. Circ Res. Jul 23 2010;107(2):233-241.

24. Olson MS, Dennis SC, DeBuysere MS, et al. The regulation of pyruvate dehydrogenase in the isolated perfused rat heart. J Biol Chem. Oct 25 1978;253(20):7369-7375.

25. Chawla A, Repa JJ, Evans RM, et al. Nuclear receptors and lipid physiology: opening the X-files. Science. Nov 30 2001;294(5548):1866-1870.

26. Huss JM, Kelly DP. Nuclear receptor signaling and cardiac energetics. Circ Res. Sep 17 2004;95(6):568-578.

27. Alvarez-Guardia D, Palomer X, Coll T, et al. PPARbeta/delta activation blocks lipid-induced inflammatory pathways in mouse heart and human cardiac cells. Biochim Biophys Acta. Feb 2011;1811(2):59-67.

28. Berger J, Moller DE. The mechanisms of action of PPARs. Annu Rev Med. 2002;53:409-435.

29. Vega RB, Huss JM, Kelly DP. The coactivator PGC-1 cooperates with peroxisome proliferatoractivated receptor alpha in transcriptional control of nuclear genes encoding mitochondrial fatty acid oxidation enzymes. Mol Cell Biol. Mar 2000;20(5):1868-1876.

30. Sato O, Kuriki C, Fukui Y, et al. Dual promoter structure of mouse and human fatty acid translocase/CD36 genes and unique transcriptional activation by peroxisome proliferator-activated receptor alpha and gamma ligands. J Biol Chem. May 3 2002;277(18):15703-15711.

31. Glosli H, Gudbrandsen OA, Mullen AJ, et al. Down-regulated expression of PPARalpha target genes, reduced fatty acid oxidation and altered fatty acid composition in the liver of mice transgenic for hTNFalpha. Biochim Biophys Acta. Jun 1 2005;1734(3):235-246.

32. Sarma S, Ardehali H, Gheorghiade M. Enhancing the metabolic substrate: PPAR-alpha agonists in heart failure. Heart Fail Rev. Nov 232010.

33. Zhou YT, Grayburn P, Karim A, et al. Lipotoxic heart disease in obese rats: implications for human obesity. Proc Natl Acad Sci U S A. Feb 15 2000;97(4):1784-1789.

34. Aasum E, Belke DD, Severson DL, et al. Cardiac function and metabolism in Type 2 diabetic mice after treatment with BM 17.0744, a novel PPAR-alpha activator. Am J Physiol Heart Circ Physiol. Sep 2002;283(3):H949-957.

35. Finck BN, Lehman JJ, Leone TC, et al. The cardiac phenotype induced by PPARalpha overexpression mimics that caused by diabetes mellitus. J Clin Invest. Jan 2002;109(1):121-130.

36. Finck BN, Han X, Courtois $M$, et al. A critical role for PPARalpha-mediated lipotoxicity in the pathogenesis of diabetic cardiomyopathy: modulation by dietary fat content. Proc Natl Acad Sci U S A. Feb 4 2003;100(3):1226-1231.

37. Watanabe K, Fujii H, Takahashi T, et al. Constitutive regulation of cardiac fatty acid metabolism through peroxisome proliferator-activated receptor alpha associated with age-dependent cardiac toxicity. J Biol Chem. Jul 21 2000;275(29):22293-22299.

38. Peters JM, Hennuyer N, Staels B, et al. Alterations in lipoprotein metabolism in peroxisome proliferator-activated receptor alpha-deficient mice. J Biol Chem. Oct 24 1997;272(43):2730727312.

39. Campbell FM, Kozak R, Wagner A, et al. A role for peroxisome proliferator-activated receptor alpha (PPARalpha) in the control of cardiac malonyl-CoA levels: reduced fatty acid oxidation rates and increased glucose oxidation rates in the hearts of mice lacking PPARalpha are associated with higher concentrations of malonyl-CoA and reduced expression of malonyl-CoA decarboxylase. J Biol Chem. Feb 8 2002;277(6):4098-4103.

40. Cheng L, Ding G, Qin Q, et al. Cardiomyocyte-restricted peroxisome proliferator-activated receptor-delta deletion perturbs myocardial fatty acid oxidation and leads to cardiomyopathy. Nat Med. Nov 2004;10(11):1245-1250.

41. Ouwens DM, Diamant $\mathrm{M}$, Fodor $\mathrm{M}$, et al. Cardiac contractile dysfunction in insulin-resistant rats fed a high-fat diet is associated with elevated CD36-mediated fatty acid uptake and esterification. Diabetologia. Sep 2007;50(9):1938-1948.

42. Carley AN, Atkinson LL, Bonen A, et al. Mechanisms responsible for enhanced fatty acid utilization by perfused hearts from type 2 diabetic $\mathrm{db} / \mathrm{db}$ mice. Arch Physiol Biochem. Apr 2007;113(2):65-75. 
43. Bonen A, Parolin ML, Steinberg GR, et al. Triacylglycerol accumulation in human obesity and type 2 diabetes is associated with increased rates of skeletal muscle fatty acid transport and increased sarcolemmal FAT/CD36. Faseb J. Jul 2004;18(10):1144-1146.

44. Kalinowska A, Gorski J, Harasim E, et al. Differential effects of chronic, in vivo, PPAR's stimulation on the myocardial subcellular redistribution of FAT/CD36 and FABPpm. FEBS Lett. Aug 6 2009;583(15):2527-2534.

45. Cresser J, Bonen A, Chabowski A, et al. Oral administration of a PPAR-delta agonist to rodents worsens, not improves, maximal insulin-stimulated glucose transport in skeletal muscle of different fibers. Am J Physiol Regul Integr Comp Physiol. Aug 2010;299(2):R470-479.

46. Choi K, Kim YB. Molecular mechanism of insulin resistance in obesity and type 2 diabetes. Korean J Intern Med. Jun 2010;25(2):119-129.

47. Van Obberghen E, Baron V, Delahaye L, et al. Surfing the insulin signaling web. Eur J Clin Invest. Nov 2001;31(11):966-977.

48. Stump CS, Henriksen EJ, Wei Y, et al. The metabolic syndrome: role of skeletal muscle metabolism. Ann Med. 2006;38(6):389-402.

49. Cho JY, Park J. Contribution of natural inhibitors to the understanding of the PI3K/PDK1/PKB pathway in the insulin-mediated intracellular signaling cascade. Int J Mol Sci. Nov 2008;9(11):2217-2230.

50. Sharma N, Arias EB, Sajan MP, et al. Insulin resistance for glucose uptake and Akt2 phosphorylation in the soleus, but not epitrochlearis, muscles of old vs. adult rats. J Appl Physiol. Jun 2010;108(6):1631-1640.

51. Park $\mathrm{S}$, Komatsu $\mathrm{T}$, Hayashi $\mathrm{H}$, et al. Calorie restriction initiated at a young age activates the Akt/PKC zeta/lambda-Glut4 pathway in rat white adipose tissue in an insulin-independent manner. Age (Dordr). Dec 2008;30(4):293-302.

52. Luiken JJ, Ouwens DM, Habets DD, et al. Permissive action of protein kinase C-zeta in insulininduced CD36- and GLUT4 translocation in cardiac myocytes. J Endocrinol. May 2009;201(2):199-209.

53. Braiman L, Alt A, Kuroki T, et al. Activation of protein kinase $C$ zeta induces serine phosphorylation of VAMP2 in the GLUT4 compartment and increases glucose transport in skeletal muscle. Mol Cell Biol. Nov 2001;21(22):7852-7861.

54. Goldberg IJ, Eckel RH, Abumrad NA. Regulation of fatty acid uptake into tissues: lipoprotein lipase- and CD36-mediated pathways. J Lipid Res. Apr 2009;50 Suppl:S86-90.

55. Schwenk RW, Luiken JJ, Bonen A, et al. Regulation of sarcolemmal glucose and fatty acid transporters in cardiac disease. Cardiovasc Res. Jul 15 2008;79(2):249-258.

56. Boudina $\mathrm{S}$, Bugger $\mathrm{H}$, Sena $\mathrm{S}$, et al. Contribution of impaired myocardial insulin signaling to mitochondrial dysfunction and oxidative stress in the heart. Circulation. Mar 10 2009;119(9):1272-1283.

57. Cho $\mathrm{H}, \mathrm{Mu} \mathrm{J}$, Kim JK, et al. Insulin resistance and a diabetes mellitus-like syndrome in mice lacking the protein kinase Akt2 (PKB beta). Science. Jun 1 2001;292(5522):1728-1731.

58. Mora A, Davies AM, Bertrand L, et al. Deficiency of PDK1 in cardiac muscle results in heart failure and increased sensitivity to hypoxia. Embo J. Sep 15 2003;22(18):4666-4676.

59. Luo J, McMullen JR, Sobkiw CL, et al. Class IA phosphoinositide 3-kinase regulates heart size and physiological cardiac hypertrophy. Mol Cell Biol. Nov 2005;25(21):9491-9502.

60. Doehner W, Rauchhaus $\mathrm{M}$, Ponikowski $\mathrm{P}$, et al. Impaired insulin sensitivity as an independent risk factor for mortality in patients with stable chronic heart failure. J Am Coll Cardiol. Sep 20 2005;46(6):1019-1026.

61. Dinh W, Futh R, Lankisch $\mathrm{M}$, et al. Cardiovascular autonomic neuropathy contributes to left ventricular diastolic dysfunction in subjects with Type 2 diabetes and impaired glucose tolerance undergoing coronary angiography. Diabet Med. Dec 222010.

62. Stahrenberg R, Edelmann F, Mende M, et al. Association of glucose metabolism with diastolic function along the diabetic continuum. Diabetologia. Jul 2010;53(7):1331-1340.

63. Coort SL, Bonen A, van der Vusse GJ, et al. Cardiac substrate uptake and metabolism in obesity and type- 2 diabetes: role of sarcolemmal substrate transporters. Mol Cell Biochem. May 2007;299(1-2):5-18. 
64. Yu C, Chen Y, Cline GW, et al. Mechanism by which fatty acids inhibit insulin activation of insulin receptor substrate-1 (IRS-1)-associated phosphatidylinositol 3-kinase activity in muscle. J Biol Chem. Dec 27 2002;277(52):50230-50236.

65. Schenk S, Saberi M, Olefsky JM. Insulin sensitivity: modulation by nutrients and inflammation. J Clin Invest. Sep 2008;118(9):2992-3002.

66. Stratford S, DeWald DB, Summers SA. Ceramide dissociates 3'-phosphoinositide production from pleckstrin homology domain translocation. Biochem J. Mar 1 2001;354(Pt 2):359-368.

67. Levy D, Garrison RJ, Savage DD, et al. Prognostic implications of echocardiographically determined left ventricular mass in the Framingham Heart Study. N Engl J Med. May 31 1990;322(22):1561-1566.

68. Efe JA, Hilcove S, Kim J, et al. Conversion of mouse fibroblasts into cardiomyocytes using a direct reprogramming strategy. Nat Cell Biol. Jan 302011.

69. Yi BA, Wernet $\mathrm{O}$, Chien KR. Pregenerative medicine: developmental paradigms in the biology of cardiovascular regeneration. J Clin Invest. Jan 4 2010;120(1):20-28.

70. Barry SP, Townsend PA. What causes a broken heart--molecular insights into heart failure. Int Rev Cell Mol Biol. 2010;284:113-179.

71. Grossman W, Jones D, McLaurin LP. Wall stress and patterns of hypertrophy in the human left ventricle. J Clin Invest. Jul 1975;56(1):56-64.

72. Opie LH, Commerford PJ, Gersh BJ, et al. Controversies in ventricular remodelling. Lancet. Jan 28 2006;367(9507):356-367.

73. Murarka S, Movahed MR. Diabetic cardiomyopathy. J Card Fail. Dec 2010;16(12):971-979.

74. Boyer JK, Thanigaraj S, Schechtman KB, et al. Prevalence of ventricular diastolic dysfunction in asymptomatic, normotensive patients with diabetes mellitus. Am J Cardiol. Apr 1 2004;93(7):870-875.

75. Zabalgoitia M, Ismaeil MF, Anderson L, et al. Prevalence of diastolic dysfunction in normotensive, asymptomatic patients with well-controlled type 2 diabetes mellitus. Am J Cardiol. Feb 1 2001;87(3):320-323.

76. Nikolaidis LA, Levine TB. Peroxisome proliferator activator receptors (PPAR), insulin resistance, and cardiomyopathy: friends or foes for the diabetic patient with heart failure? Cardiol Rev. May-Jun 2004;12(3):158-170.

77. Barouch LA, Berkowitz DE, Harrison RW, et al. Disruption of leptin signaling contributes to cardiac hypertrophy independently of body weight in mice. Circulation. Aug 12 2003;108(6):754-759.

78. Yang J, Sambandam N, Han X, et al. CD36 deficiency rescues lipotoxic cardiomyopathy. Circ Res. Apr 27 2007;100(8):1208-1217.

79. Glenn DJ, Wang F, Nishimoto $M$, et al. A murine model of isolated cardiac steatosis leads to cardiomyopathy. Hypertension. Feb 2011;57(2):216-222.

80. Condorelli G, Drusco A, Stassi G, et al. Akt induces enhanced myocardial contractility and cell size in vivo in transgenic mice. Proc Natl Acad Sci U S A. Sep 17 2002;99(19):12333-12338.

81. Shioi T, McMullen JR, Kang PM, et al. Akt/protein kinase B promotes organ growth in transgenic mice. Mol Cell Biol. Apr 2002;22(8):2799-2809.

82. Fang CX, Dong F, Thomas DP, et al. Hypertrophic cardiomyopathy in high-fat diet-induced obesity: role of suppression of forkhead transcription factor and atrophy gene transcription. Am J Physiol Heart Circ Physiol. Sep 2008;295(3):H1206-H1215.

83. Shiomi $\mathrm{T}$, Tsutsui $\mathrm{H}$, Ikeuchi $\mathrm{M}$, et al. Streptozotocin-induced hyperglycemia exacerbates left ventricular remodeling and failure after experimental myocardial infarction. J Am Coll Cardiol. Jul 2 2003;42(1):165-172.

84. Fischer VW, Barner HB, Larose LS. Pathomorphologic aspects of muscular tissue in diabetes mellitus. Hum Pathol. Dec 1984;15(12):1127-1136.

85. Martinez DA, Guhl DJ, Stanley WC, et al. Extracellular matrix maturation in the left ventricle of normal and diabetic swine. Diabetes Res Clin Pract. Jan 2003;59(1):1-9.

86. Hirotani S, Otsu K, Nishida K, et al. Involvement of nuclear factor-kappaB and apoptosis signalregulating kinase 1 in G-protein-coupled receptor agonist-induced cardiomyocyte hypertrophy. Circulation. Jan 29 2002;105(4):509-515.

87. Nishida $M$, Maruyama $Y$, Tanaka R, et al. $G$ alpha(i) and $G$ alpha(o) are target proteins of reactive oxygen species. Nature. Nov 23 2000;408(6811):492-495. 
88. Molkentin JD. Calcineurin-NFAT signaling regulates the cardiac hypertrophic response in coordination with the MAPKs. Cardiovasc Res. Aug 15 2004;63(3):467-475.

89. Kandasamy AD, Chow AK, Ali MA, et al. Matrix metalloproteinase-2 and myocardial oxidative stress injury: beyond the matrix. Cardiovasc Res. Feb 1 2010;85(3):413-423.

90. Goser S, Andrassy M, Buss SJ, et al. Cardiac troponin I but not cardiac troponin T induces severe autoimmune inflammation in the myocardium. Circulation. Oct 17 2006;114(16):16931702.

91. Tsutsui H, Kinugawa S, Matsushima S. Mitochondrial oxidative stress and dysfunction in myocardial remodelling. Cardiovasc Res. Feb 15 2009;81(3):449-456.

92. Chen L, Knowlton AA. Mitochondria and heart failure: new insights into an energetic problem. Minerva Cardioangiol. Apr 2010;58(2):213-229.

93. Li CJ, Zhang QM, Li MZ, et al. Attenuation of myocardial apoptosis by alpha-lipoic acid through suppression of mitochondrial oxidative stress to reduce diabetic cardiomyopathy. Chin Med J (Engl). Nov 5 2009;122(21):2580-2586.

94. Cai L, Li W, Wang G, et al. Hyperglycemia-induced apoptosis in mouse myocardium: mitochondrial cytochrome C-mediated caspase-3 activation pathway. Diabetes. Jun 2002;51(6):1938-1948. 


\title{
CHAPTER 3
}

\section{Protein kinase D is essential for contrac- tion-induced glucose uptake, but is not involved in fatty acid uptake into cardi- omyocytes}

\author{
Ellen Dirkx ${ }^{1}$, Robert W. Schwenk ${ }^{1}$, Arend Bonen ${ }^{2}$, Guillaume J.J.M. van Eys ${ }^{1}$, \\ Jan F.C. Glatz ${ }^{1}$, and Joost J.F.P. Luiken ${ }^{1}$
}

\footnotetext{
${ }^{1}$ Department of Molecular Genetics and Cardiovascular Research Institute Maastricht (CARIM), Maastricht University, the Netherlands,

${ }^{2}$ Department of Human Health and Nutritional Sciences, College of Biological Sciences, University of Guelph, Ontario, Canada.
} 


\begin{abstract}
Increased contractile performance enhances substrate uptake into cardiomyocytes via translocation of both the glucose transporter GLUT4 and the long-chain fatty acid (LCFA) transporter CD36 from intracellular stores to the sarcolemma. Additionally, contraction stimulation activates both the signaling enzymes AMP-activated protein kinase (AMPK) and protein kinase-D1 (PKD). While AMPK has been implicated in contraction-induced GLUT4 and CD36 translocation in the heart, the precise role of PKD in these processes is not known. To study this, we triggered contractions in cardiomyocytes by electric field stimulation (EFS). First, the role of PKD in GLUT4 and CD36 translocation was defined. RNA interference or pharmacological inhibition of PKD abolished EFS-induced GLUT4 translocation but not CD36 translocation, whereas AMPK silencing abrogated both translocation events, indicating that, unlike AMPK, PKD is selectively involved in glucose uptake. Secondly, we determined upstream factors in PKD activation. Contraction-produced ROS was shown to activate PKD via death-activated protein kinase (DAPK). ROS scavenging markedly reduced EFS-induced glucose but not LCFA uptake, and DAPK silencing abrogated EFSinduced GLUT4 but not CD36 translocation. Finally, possible links between PKD and AMPK signaling were investigated. PKD silencing or interruption of the ROS-DAPKPKD pathway did not affect AMPK activation. Reciprocally, AMPK silencing had no effect on PKD activation. In conclusion, we disclose a novel contraction-induced ROS-DAPK-PKD pathway in cardiomyocytes that induces GLUT4 translocation independently of AMPK. Thus, unlike AMPK, PKD is selectively involved in glucose and not LCFA uptake, and therefore may provide a suitable target to shift cardiac substrate preference away from LCFA towards glucose.
\end{abstract}




\section{Introduction}

Cardiac substrate utilization is predominantly regulated by GLUT4 and CD36, the main cardiac transporters for glucose and long-chain fatty acids (LCFA), respectively. ${ }^{1}$ Besides being present at the sarcolemma, GLUT4 and CD36 are also stored in endosomal compartments, from where these transporters can be translocated to the sarcolemma. GLUT4 translocation is known to be a vesicle-mediated process depending on, a.o., coat- and SNARE proteins. Recently, both groups of proteins have also been found to mediate CD36 translocation. ${ }^{2}$ Alterations in contractile performance are an important denominator of cardiac substrate utilization. Increased contractile activity enhances the demands for glucose and LCFA, and accordingly, GLUT4 and CD36 have been found to translocate to the sarcolemma under this condition. ${ }^{1,3}$

The signaling pathways involved in contraction-induced GLUT4 and CD36 translocation in the heart have been incompletely charted. In this respect, it is known that contraction-signaling is under control of the cardiac energy status, and particularly sensitive to increases in intracellular levels of AMP. Specifically, AMP binds to the regulatory $Y$-subunit of the heterotrimeric enzyme AMPK-activated protein kinase (AMPK). This binding results in partial unfolding of AMPK and exposing a Thr172 site within the catalytic $\alpha$-subunit to an activating phosphorylation by the upstream kinase LKB1. ${ }^{4}$ Both LKB1 and AMPK have been shown to be necessary for GLUT4 and CD36 translocation in cardiomyocytes. ${ }^{5,6}$ However, it is not known whether there are also kinases that are differentially involved in contraction-induced GLUT4 and CD36 translocation in the heart.

Another contraction-activated kinase in the heart is protein kinase D (PKD). Contraction-stimulation has recently been found to autophosphorylate PKD at Ser916, resulting in increased phosphorylation of established PKD target proteins. ${ }^{7}$ PKD is the founding member of the PKD family, a novel family of protein kinase-C related Ser/Thr kinases. PKD is associated with a number of cellular processes. For instance, PKD is involved in translocation of secretory proteins from the Golgi to the plasma membrane in secretory cells. ${ }^{8}$ Additionally, PKD links increased production of reactive oxygen-species (ROS) to inflammatory responses through activation of nuclear factor к-B. ${ }^{9}$ Recently, we discovered that PKD is linked to contraction-induced glucose uptake. Namely, agents (i.e., staurosporine and calphostin-C) that blocked PKD activation also abolished contraction-induced GLUT4 translocation. ${ }^{7}$ However, because these agents additionally inhibit PKC members and a variety of other kinases, the role of PKD in contraction-induced GLUT4 translocation awaits more definite proof. Furthermore, the role of PKD in contraction-induced CD36 translocation is unknown. 
In this study, we examined the role of PKD in glucose and LCFA uptake in contracting cardiomyocytes, and compared the effects of PKD with those of AMPK in inducing GLUT4 and CD36 translocation. Furthermore, we mapped the signaling events upstream of PKD, focusing on ROS as candidate second messenger. Finally, we investigated whether or not PKD signaling converges with AMPK signaling in regulation of contraction-induced substrate uptake in the heart. For manipulation of PKD and AMPK expression, we used the cardiac cell line HL1 in which we knocked down PKD and AMPK via siRNA technology. For inhibition and activation of ROS signaling, we treated primary rat cardiomyocytes with $\mathrm{N}$-(2-mercaptopropionyl)glycine (MPG) and $\mathrm{H}_{2} \mathrm{O}_{2}$, respectively. We conclude that upon activation by contraction-produced ROS, PKD induces GLUT4, but not CD36, translocation in an AMPK-independent pathway. 


\section{Materials and Methods}

\section{Animals}

Male Lewis rats (200-250 g) were obtained from Charles River. All animals were maintained at the Experimental Animal Facility of Maastricht University. The investigation conforms to the Guide for the Care and Use of Laboratory Animals published by the US National Institutes of Health (NIH Publication No. 85-23, revised 1996).

\section{Materials}

Lipofectamine-2000, N-(2-mercaptopropionyl)glycine (MPG) and 5-(and -6)-carboxy$2^{\prime}, 7^{\prime}$-dichlorodihydrofluorescein diacetate (carboxy-H2-DCFDA), were purchased from Invitrogen (San Diego, USA). Liberase blendzyme 1, complete protease inhibitor and PhosSTOP phosphatase inhibitor were obtained from Roche Diagnostics (Indianapolis, IN). IGEPAL CA-630 was from Sigma. Small-interfering RNAs (siRNAs) against PKD (GGAGGGUGAUCUCAUUGAAtt), AMPK-alpha-2 catalytic subunit (GCACGGUCAAGUUUUGAUUtt) and DAPK1 (GCAAGAAACAUUAGCGAAUtt), were obtained from Ambion Applied Biosystems (Austin, USA). The plasmid coding for GLUT4myc was kindly provided by Dr. J. Eckel (German Diabetes Center, Düsseldorf, Germany). Green fluorescent protein (GFP) eukaryotic expression vector pEGFP-N1 was from Clontech (Leusden, Netherlands). Antibodies directed against phosphoPKD/PKC- $\mu$ (Ser916), phospho-AMPK (Thr172), GAPDH, caveolin 3 and the Myc epitope were obtained from Cell Signaling (Beverly, USA) and the antibody directed against DAP kinase-1 was purchased from Sigma-Aldrich (St. Louis, USA). The antibody directed against GLUT4 was obtained from Santa Cruz Biotechnology (Santa Cruz, USA) and the antibody against mouse CD36 was from Chemicon (Billerica, USA). Protein G Sepharose beads were from GE Healthcare (Eindhoven, Netherlands). All culture dishes, pipets, tubes and containers used, were from Greiner BioOne (Alphen aan de Rijn, the Netherlands).

\section{Experimental procedures on HL-1 atrial cardiomyocytes}

HL-1 cells were kindly provided by Dr. Claycomb (Louisiana State University, New Orleans, USA) and cultured as previously described (2). HL1-cells were transfected with non-coding (nc)-, PKD-, AMPK- or DAPK1-siRNA and cotransfected with pCMVGLUT4myc (in case of sarcolemmal GLUT4 detection). Transfection of siNA was 
done by mixing 200 pmol siRNA with $8 \mu$ l Lipofectamine-2000 per well (6-well plate) in antibiotic- and norepinephrine-free culture medium. After $6 \mathrm{~h}$, the medium was changed to regular culture medium. After $48 \mathrm{~h}$ the medium was changed to FCSand norepinephrine-free medium overnight. Subsequently, cells were subjected to electric field-stimulation (EFS) for inducing controlled cellular contractions (in case of substrate uptake studies and transporter translocation assays), or subjected to oligomycin treatment (in case of verification of loss of signaling due to silencing of each of the three kinases). In case of EFS, the C-Pace stimulator from lonOptix (Milton, USA) was used at a setting of $40 \mathrm{~V}, 5 \mathrm{~ms}$ pulse duration and $2 \mathrm{~Hz}$ during $30 \mathrm{~min}$. Immediately therafter, sarcolemmal contents of CD36, GLUT4myc or uptake of glucose and LCFA were measured. Sarcolemmal GLUT4myc and CD36 were detected by cell staining with an HRP-linked secondary antibody as previously described. ${ }^{2}$ Cellular uptake of $\left[{ }^{3} \mathrm{H}\right]$ deoxyglucose and $\left[{ }^{14} \mathrm{C}\right]$ palmitate were determined after $10 \mathrm{~min}$ as cell-associated radioactivity. ${ }^{2}$ In case of oligomycin, cells were incubated for $30 \mathrm{~min}$ at $1 \mu \mathrm{M}$, and then lysed in Laemmli sample buffer for detection of signaling actions via Western blotting with phosphospecific actions. To microscopically track GLUT4 translocation, murine GLUT4 CDNA was ligated into pEGFP-N1. The resulting plasmid was transfected into HL1 cells using Lipofectamine-2000. Transfected cells were observed and photographed by a Leica TCS SPE confocal scanning microscope.

\section{Isolation and treatment of adult rat cardiomyocytes}

Cardiomyocytes were isolated from male Lewis rats (200-250 g) using a Langendorff perfusion system and a Krebs Henseleit bicarbonate medium equilibrated with a $95 \% \mathrm{O}_{2} / 5 \% \mathrm{CO}_{2}$ gas phase at $37^{\circ} \mathrm{C}^{10}$ and subsequently incubated at $37^{\circ} \mathrm{C}$ with continuous shaking. For induction of contraction, cardiomyocytes were subjected to EFS at $40 \mathrm{~V}, 5 \mathrm{~ms}$ pulse duration, $4 \mathrm{~Hz}$ during $6 \mathrm{~min}$ using the C-Pace stimulator. Additionally, cardiomyocytes were incubated for $15 \mathrm{~min}$ at $37^{\circ} \mathrm{C}$ with $5 \mu \mathrm{M}$ oligomycin. In case of addition of pharmacological inhibitors, compounds were added $30 \mathrm{~min}$ prior to stimulation. Immediately after stimulation, a mixture of $\left[{ }^{3} \mathrm{H}\right]$ deoxyglucose and $\left[{ }^{14} \mathrm{C}\right.$ ]palmitate was added during $5 \mathrm{~min}$, and uptake of these substrates was determined as pelletable radioactivity as previously described. ${ }^{10}$

\section{Immunoprecipitation}

Cardiomyocytes were pelleted and incubated for $1 \mathrm{~h}$ in ice-cold lysis buffer (50 $\mathrm{mmol} / \mathrm{L}$ Tris- $\mathrm{HCl}$ ( $\mathrm{pH}=7.4), 150 \mathrm{mmol} / \mathrm{L} \mathrm{NaCl}, 1 \%$ IGEPAL CA-630, 0.5\% sodium deoxycholate, $0.1 \%$ SDS, complete protease inhibitor and PhosSTOP phosphatase inhibitor. Cell lysates were pelleted and the supernatant fractions were incubated with 
antibody $\left(5 \mu \mathrm{g}\right.$ ) overnight at $4^{\circ} \mathrm{C} .50 \mu \mathrm{l}$ of Protein $\mathrm{G}$ Sepharose beads was added and incubations were continued for $1 \mathrm{~h}$ at $4^{\circ} \mathrm{C}$. The beads were collected by pulse centrifugation and washed. The final pellet was resuspended in $30 \mu \mathrm{l}$ Laemmli sample buffer and heated to 95 o $\mathrm{C}$ for $5 \mathrm{~min}$. The beads were pelleted by centrifugation and the supernatant was used for Western blot analysis.

\section{Immunoblotting}

Proteins were separated by SDS-PAGE on 4-12\% Bis-Tris Criterion XT precast gels (Bio-rad, California, USA), and transferred to nitrocellulose membranes for Western blotting. The protein bands were visualized as previously described ${ }^{7}$

\section{Flow cytometry analysis}

To measure generation of reactive oxygen species, cardiomyocytes were incubated for 30 min with $10 \mu \mathrm{M}$ 5(6)-carboxy-2,7-diclorodihydrofluorescein diacetate (carboxy- $\mathrm{H}_{2}$-DCFDA). Afterwards, the cardiomyocytes were washed with PBS and fluorescence was analysed by flow cytometry.

\section{Statistics}

All data are presented as means \pm SEM. Statistical analysis was performed by using Student's t-test or ANOVA (including Newman-Keuls Multiple Comparison Test) and statistical analysis software Prism 4 (GraphPad Software, Inc.). A P value of $<0.05$ was considered statistically significant.

\section{Results}

Involvement of PKD and AMPK in contraction-induced GLUT4 and CD36 translocation and glucose and LCFA uptake.

HL1-cardiomyocytes were chosen as model to study the influence of PKD and AMPK on cardiac substrate uptake. This cell line can be readily manipulated with siRNA technology for protein silencing. ${ }^{2}$ Additionally, HL1-cells are capable of contracting in an electric field. ${ }^{2}$ Furthermore, we verified in HL1 cells that GLUT4 translocation is entirely responsible for stimulus-induced glucose uptake and CD36 translocation for stimulus-induced LCFA uptake. ${ }^{2}$ Fig. 1A shows that treatment of HL1 cells with siRNA against PKD or AMPK resulted in a $\geq 40 \%$ specific loss of protein expression of each these respective kinases. Oligomycin, an inhibitor of mitochondrial $F_{1} F_{0^{-}}$ 
ATPase, has been shown to stimulate AMPK and PKD activation in a similar manner as does contraction, ${ }^{3,7}$ and was used in these experiments as stimulus to verify whether the silencing of both kinases would also abrogate their activation. Indeed, silencing of PKD resulted in a complete loss of oligomycin-induced PKD-Ser919 phosphorylation, and silencing of AMPK led to a complete loss of oligomycininduced AMPK-Thr172 phosphorylation. (Fig. 1A). Hence, activation of both kinases is abolished due to reduced synthesis. Furthermore, PKD silencing did not affect AMPK activation and, conversely, AMPK silencing did not influence PKD activation, indicating that these kinases are operable in separate and independent pathways (Fig. 1A). With respect to substrate transporters, PKD silencing abrogated EFSinduced GLUT4 translocation, but not CD36 translocation (Fig. 1B). Additional evidence for the involvement of PKD in contraction-induced GLUT4 translocation was obtained in microscopical studies using a GLUT4-GFP hybrid protein: GLUT4-GFP is shown to be translocated to the sarcolemma upon EFS, and this is abolished by PKD silencing (Fig. 1C). Conversely to PKD, AMPK knockdown of cardiomyocytes inhibited EFS-induced translocation of both GLUT4 and CD36 (Fig. 1B). 
Figure 1

A

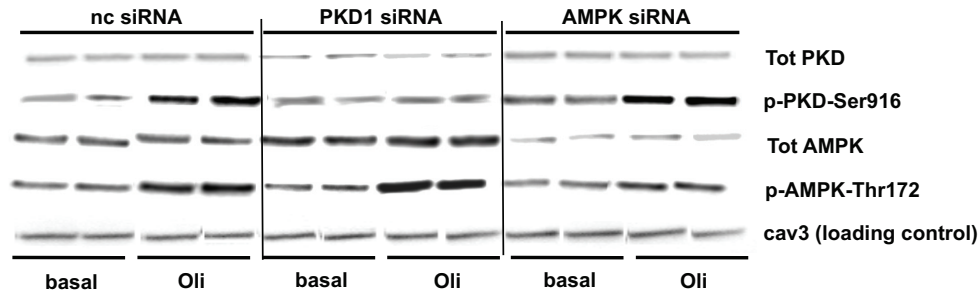

B
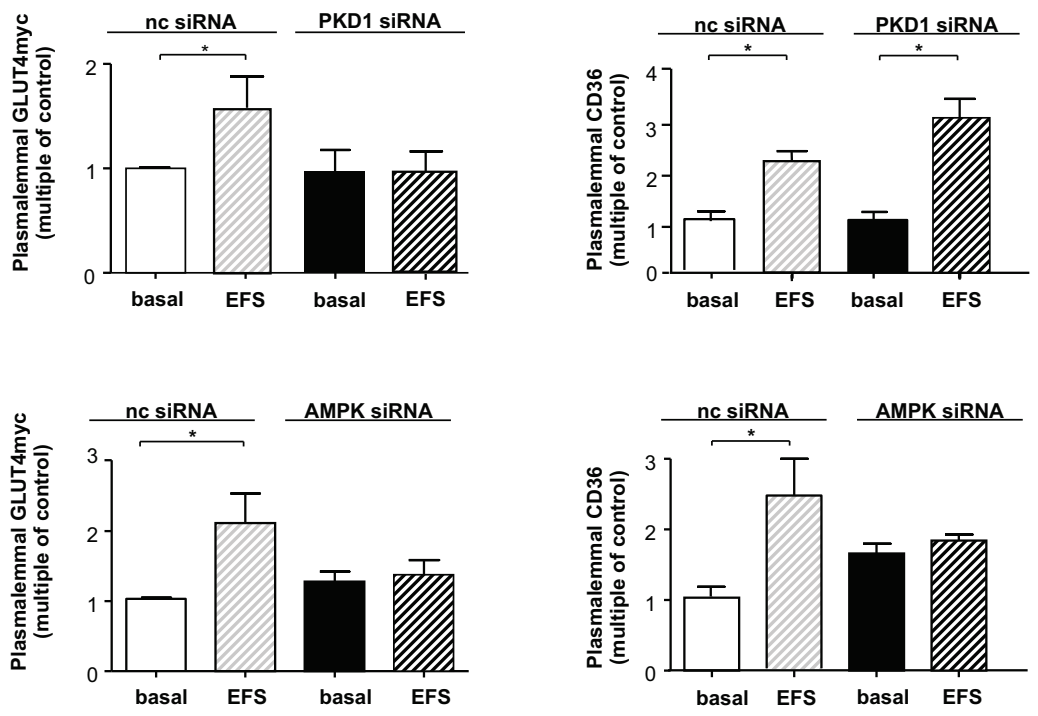

C

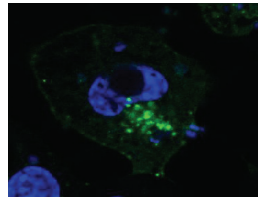

nc siRNA

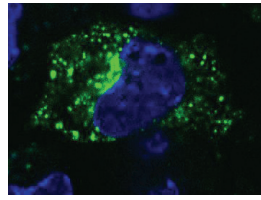

nc siRNA + EFS

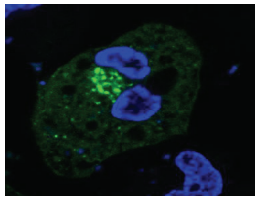

PKD1 siRNA

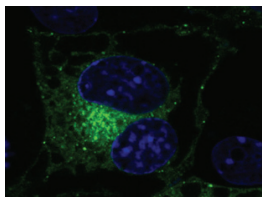

PKD1 siRNA + EFS

Fig. 1 PKD silencing in HL1-cardiomyocytes abolishes contraction-induced GLUT4 translocation and glucose uptake but not AMPK activation or CD36 translocation and LCFA uptake. HL1-cardiomyocytes were transfected with non-coding (nc)-, PKD- or AMPK-siRNA (and cotransfected with pCMV-GLUT4myc in case of sarcolemmal GLUT4 detection). After $48 \mathrm{~h}$, cells were treated with oligomycin (oli; $1 \mu \mathrm{M}, 30 \mathrm{~min}$ ) or subjected to electric field-stimulation (EFS) for $30 \mathrm{~min}$. Subsequently, (A) total and phosphorylated PKD and AMPK were detected by immunoblotting $(n=4)$, and (B) sarcolemmal GLUT4myc and CD36 contents were detected by a fluorometric assay following cell staining with an HRP-linked secondary antibody $(n=4)$. (C) For microscopical visualization of GLUT4 translocation, cells were transfected with non-coding (nc)-, PKD-siRNA, and cotransfected with GLUT4-GFP, and after $48 \mathrm{~h}$ subjected to EFS. Translocation of 
GLUT4-GFP was detected via immunofluorescence microscopy. Nuclei are stained in blue (DAPI). Only in the nc-siRNA-transfected cells subjected to EFS, GLUT4-GFP hybrid proteins move out to the plasma membrane. A representative experiment out of 3 is displayed.

We also investigated the role of PKD in contraction-induced glucose and LCFA uptake into primary cardiomyocytes, using the staurosporin-derivative Ro318220, which has been routinely used to inhibit PKD activation. ${ }^{11}$ Treatment of these primary cardiomyocytes with Ro318220 blocks PKD-Ser916 phosphorylation and EFSinduced glucose uptake, but not AMPK-Thr172 phosphorylation nor EFS-induced LCFA uptake (Fig. 2). These findings are in agreement with the abolishment of EFSinduced GLUT4 translocation, but not CD36 translocation, in PKD-silenced HL1 cells. Taken together, PKD is specifically involved in GLUT4-mediated glucose uptake, whereas AMPK is necessary for both GLUT4 mediated glucose uptake and CD36mediated LCFA uptake.

Figure 2

A

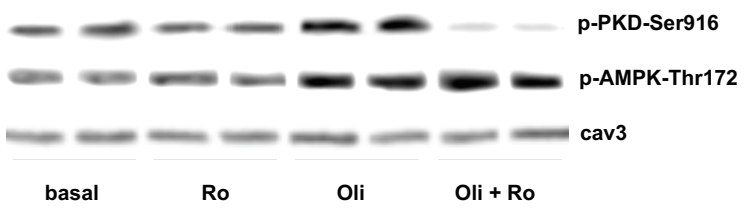

B
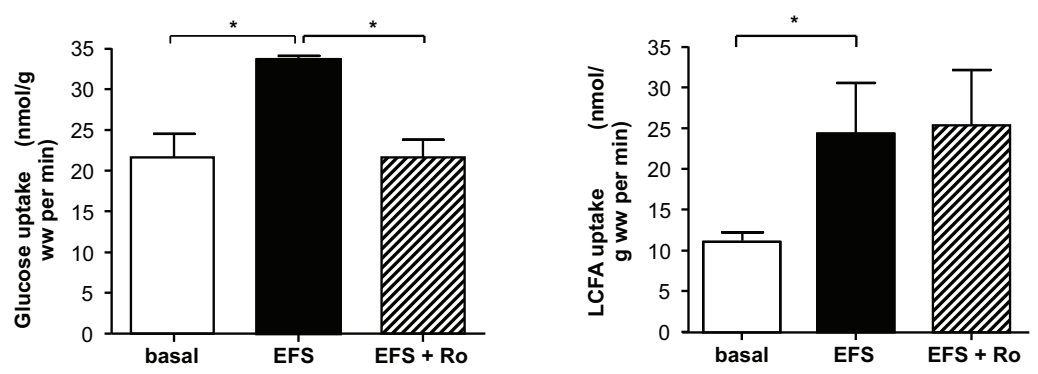

Fig. 2 PKD inhibition by Ro318220 in primary cardiomyocytes confirms that PKD is involved in contractioninduced glucose, but not LCFA uptake. Primary rat cardiomyocytes were preincubated in the absence or presence of Ro318220 (10 $\mu \mathrm{M} ; 30 \mathrm{~min}$ ), and subsequently treated with oligomycin (oli; $5 \mu \mathrm{M} ; 15 \mathrm{~min}$ ) or subjected to $4 \mathrm{~Hz}$-electrical field stimulation (EFS) prior to measurement of (A) PKD-Ser916 and AMPKThr172 phosphorylation, or (B) measurement of initial $(5 \mathrm{~min})$ uptake of $\left[{ }^{3} \mathrm{H}\right]$ deoxyglucose and $\left[{ }^{14} \mathrm{C}\right]$ palmitate $(\mathrm{n}=4)$. 
Upstream events of PKD in contraction-induced GLUT4 translocation and glucose uptake.

We hypothesized that ROS presents an upstream effector of the PKD-GLUT4 pathway. In isolated cardiomyocytes, EFS significantly increased ROS formation (Fig. 3A). Oligomycin was also used as positive control for ROS production, ${ }^{12}$ and indeed stimulated ROS production in these cells (Fig. 3A). Subsequently, we linked increased ROS production to PKD activation in these cardiomyocytes, because the ROSinducing compound $\mathrm{H}_{2} \mathrm{O}_{2}$ markedly enhanced PKD-Ser916 phosphorylation (Fig. 3B). To further establish the relationship between contraction-induced ROS production, PKD activation, and glucose uptake, we used the ROS scavenger MPG. ${ }^{13}$ MPG markedly decreased oligomycin-induced PKD-Ser916 phosphorylation, but did not affect AMPK-Thr172 phosphorylation (Fig. 3C). Also, MPG largely blocked EFS-induced glucose uptake, without affecting EFS-induced LCFA uptake (Fig. 3D). Hence, ROS is involved in contraction-induced PKD activation and glucose uptake, but does not appear to play a role in contraction-induced AMPK activation or LCFA uptake. 
Figure 3

A

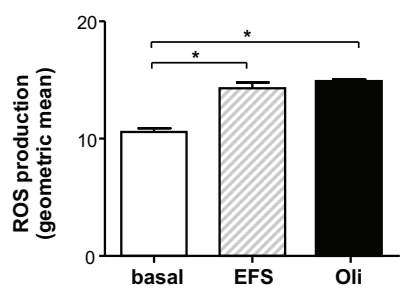

C

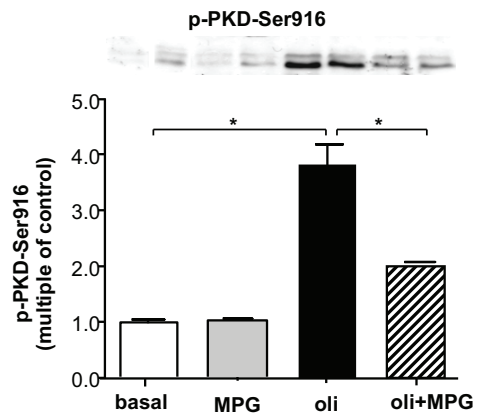

D

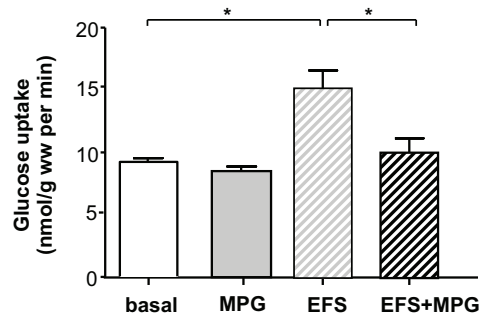

B
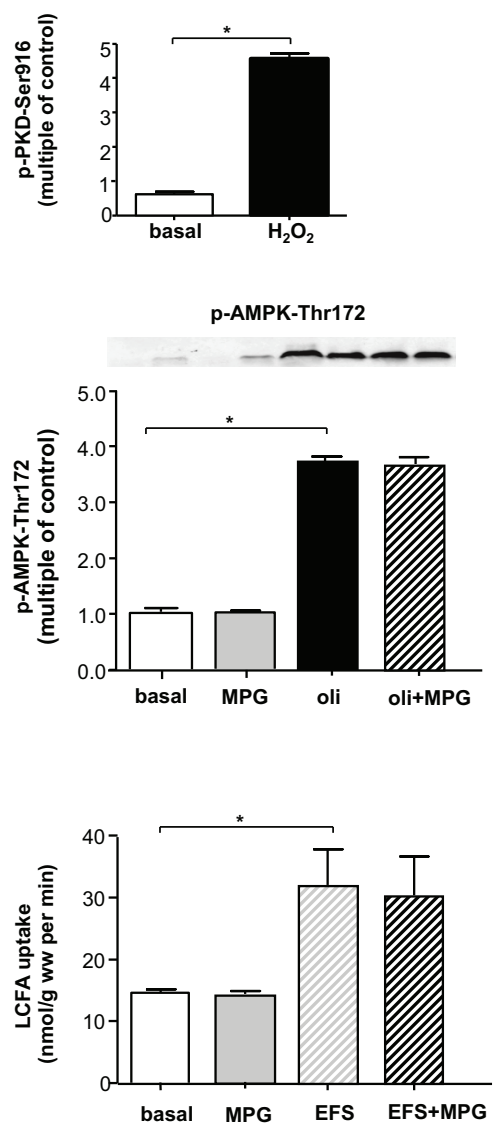

Fig. 3 ROS signaling is involved in contraction-induced glucose uptake. (A,B) Primary rat cardiomyocytes were treated with oligomycin (oli; $5 \mu \mathrm{M} ; 15 \mathrm{~min}$ ), $4 \mathrm{~Hz}$-electric field-stimulation (EFS), or with the ROS donor $\mathrm{H}_{2} \mathrm{O}_{2}(10 \mu \mathrm{M} ; 10 \mathrm{~min})$ prior to (A) measurement of ROS production or (B) measurement of autophosphorylation at Ser916 (reflecting PKD activation). (C,D) Primary rat cardiomyocytes were preincubated in absence or presence of the ROS scavenger MPG, and subsequently treated with oli or EFS prior to (C) Western detection of PKD-Ser916- and AMPK-Thr172 phosphorylation $(n=4)$, or (D) measurement of initial uptake of $\left[{ }^{3} \mathrm{H}\right]$ deoxyglucose and $\left[{ }^{14} \mathrm{C}\right]$ palmitate $(n=4)$. * Statistically different $(p<0.05)$.

The evidence that ROS is involved in contraction-induced PKD-mediated GLUT4 translocation allows to speculate that ROS-activated kinases are directly activating PKD and thereby induce GLUT4 translocation. One of the kinases that is activated by oxidative stress is death-activated protein kinase (DAPK). ${ }^{14}$ Furthermore in HEK cells, 
oxidative stress triggered physical interaction of DAPK with PKD. ${ }^{14}$ However, DAPK has never been linked to cardiac glucose utilization. Here, we investigated whether in cardiomyocytes DAPK physically interacts with PKD during contraction, and whether it mediates GLUT4 translocation. Indeed, EFS induced a physical association of DAPK and PKD as shown in reciprocal immunoprecipitation analyses (Fig. 4A). Since co-immunoprecipitation was absent in non-stimulated cardiomyocytes, the interaction between DAPK and PKD is due to contraction stimulation. Furthermore, in DAPK immunoprecipitates the increase in PKD content due to EFS is accompanied by a similar increase in Ser916-phosphorylated PKD (Fig. 4A), suggesting that binding of DAPK to PKD upon the onset of contraction leads to PKD activation. Knockdown of DAPK (64 $\pm 7 \%$ ) abrogated PKD activation (Fig. 4B), EFS-induced GLUT4 translocation (Fig. 4C) and EFS-induced GLUT4-GFP translocation (Fig. 4D). This indicates that DAPK is necessary for contraction-induced PKD activation and subsequent GLUT4 translocation. In contrast, CD36 translocation was not affected by DAPK knockdown (Fig. 4C). Combined, these data position ROS and DAPK upstream of PKD activation and demonstrate that the ROS-DAPK-PKD signaling selectively controls GLUT4-mediated glucose uptake. 
Figure 4

A IP: DAPK
basal EFS

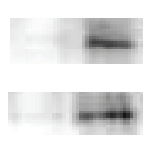

IB: PKD

IP: PKD

basal EFS

IB: p-PKD-Ser916

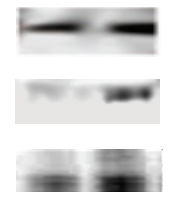

IB: PKD

IB: p-PKD-Ser916

IB: DAPK

IB: DAPK

B

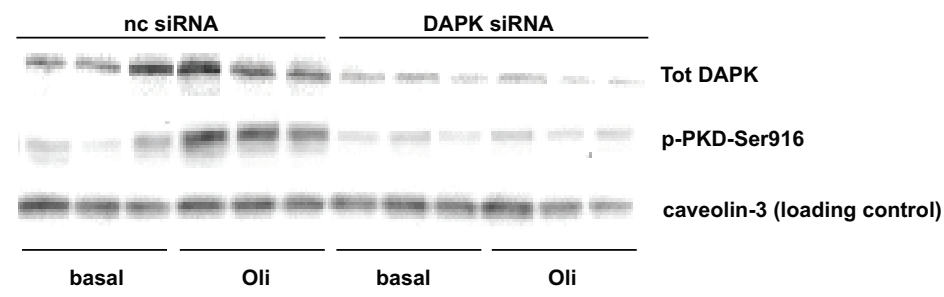

C
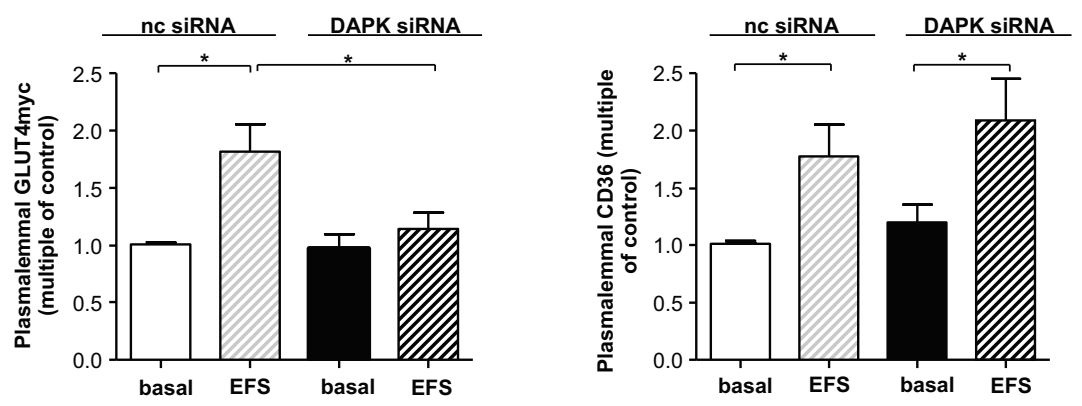

\section{D}

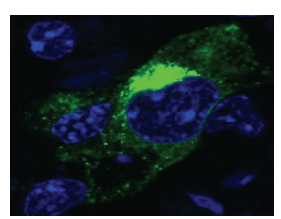

nc siRNA

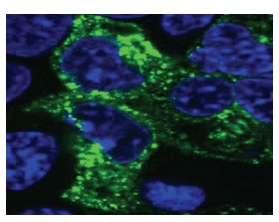

nc siRNA + EFS

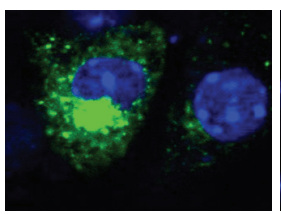

DAPK SIRNA

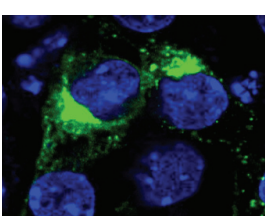

DAPK SIRNA + EFS

Fig. 4 DAPK is involved in PKD activation and contraction-induced GLUT4 translocation. (A) Primary rat cardiomyocytes were treated with or without EFS, and subsequently used for immunoprecipitation (IP) with monoclonal anti-DAPK- or PKD- antibodies, after which both the DAPK-immunoprecipitate and the PKD immunoprecipitate were used for immunoblotting (IB) against total PKD protein expression, phospho-PKD-Ser916 and total DAPK protein expression. Representative blots are shown ( $n=3)$. (B,C) HL1cardiomyocytes were transfected with non-coding (nc)- or DAPK-siRNA and cotransfected with pCMVGLUT4myc (in case of sarcolemmal GLUT4 detection) and after $48 \mathrm{~h}$ treated with oligomycin (oli; $1 \mu \mathrm{M}$, $30 \mathrm{~min}$ ) or subjected to electric field-stimulation (EFS). (B) DAPK expression and PKD-Ser916 phosphorylation were detected by IB $(n=4)$, and (C) sarcolemmal GLUT4myc and CD36 by a fluorometric assay 
following cell staining with an HRP-linked secondary antibody $(n=4)$. * Statistically different $(p<0.05)$. (D) Additionally, nc-siRNA-treated or DAPK-silenced cells were cotransfected with GLUT4 GFP, and after $48 \mathrm{~h}$ subjected to EFS for immunofluoresence detection of GLUT4 translocation. Nuclei are stained in blue (DAPI). Only in the nc-siRNA-transfected cells subjected to EFS, GLUT4-GFP hybrid proteins move out to the plasma membrane. A representative experiment out of 3 is displayed.

\section{Discussion}

This study was aimed at investigating the role of PKD, in relation to AMPK, in regulating substrate uptake into contracting cardiomyocytes, and also aimed at elucidating the upstream events responsible for contraction-induced PKD activation. The presented results yielded the following novel insights: (i) Unlike AMPK, being involved in both contraction-induced glucose and LCFA uptake, PKD is specifically involved in contraction-induced glucose uptake. (ii) The upstream events in contraction-induced PKD activation include ROS production and binding of PKD to DAPK. (iii) PKD and AMPK are activated via separate contraction-induced pathways, but activation of both kinases is necessary for GLUT4 translocation. Below, we will discuss the evidence for these conclusions in further detail.

\section{PKD is specifically involved in contraction-induced glucose uptake.}

Previously, we found that staurosporine and calphostin- $\mathrm{C}$, compounds that inhibit PKD, blocked contraction-induced GLUT4 translocation and glucose uptake in primary rat cardiomyocytes. ${ }^{7}$ However, these compounds, being non-selective pan-PKC inhibitors, do not allow to discriminate between PKCs and PKD in mediating contraction-induced GLUT4 translocation. Furthermore, it was not investigated in that study whether pharmacological PKC/PKD inhibition affected contraction-induced CD36 translocation and LCFA uptake.

In the present study we obtained two lines of evidence that PKD is not involved in CD36 translocation, and hence specifically mediates contraction-induced GLUT4 translocation: (i) In HL1 cardiomyocytes PKD silencing inhibited contraction-induced glucose uptake, but had no effect on contraction-induced LCFA uptake. (ii) In primary cardiomyocytes pharmacological PKD inhibition by Ro318220 inhibited contraction-induced glucose uptake but not LCFA uptake. Furthermore, preliminary experiments in cardiomyocytes from cardiac-specific constitutively active PKD overexpression mice (from Prof. E.N. Olson, University of Texas Southwestern Medical Center, Dallas, USA) show that glucose uptake, but not LCFA uptake was upregulated (data not shown). On the other hand, AMPK activation is involved in both GLUT4 
and CD36 translocation in cardiomyocytes. First, in rat cardiomyocytes, AMPKactivating stimuli, induce translocation of both GLUT4 and CD36, ${ }^{1,3}$ and in cardiomyocytes from dominant-negative AMPK expressing mice, ${ }^{15}$ contraction-induced glucose and LCFA uptake is abolished. ${ }^{6}$ Moreover, in the present study using the same HL1 cell passages in which PKD was silenced, AMPK silencing abolished both contraction-induced GLUT4 and CD36 translocation. Taken together, both activated AMPK and PKD induce GLUT4-mediated glucose uptake. However, only PKD is able to selectively stimulate glucose uptake, and therefore PKD uniquely can shift the cardiac substrate preference towards glucose.

\section{Upstream events in contraction-induced PKD activation.}

It has been shown that the induction of contraction enhances ROS formation in cardiomyocytes, ${ }^{16}$ and that ROS scavengers inhibit contraction-induced glucose uptake. $^{17,18}$ Furthermore, PKD is known to be activated by mitochondriallyproduced ROS. ${ }^{9}$ Combining the findings from these different studies allows the speculation that ROS is involved in contraction-induced PKD-mediated GLUT4 translocation in the heart. Indeed, we confirmed that contraction enhances ROS formation in cardiomyocytes, after which we observed that induction of ROS production in cardiomyocytes results in Ser916 phosphorylation, and hence, activation of PKD. Subsequently, we established a causal link between ROS formation and PKD activation and GLUT4 translocation based on the ability of pharmacological ROS scavenging to inhibit contraction-induced PKD activation and glucose uptake.

In search of an upstream kinase mediating ROS-induced PKD activation in cardiomyocytes, and subsequent GLUT4 translocation, we speculated on the involvement of DAPK. Namely, DAPK has been shown in HEK-cells to physically interact with and to activate PKD in response to oxidative stress. ${ }^{14}$ However, DAPK is mainly known as a tumor suppressor which expression is greatly reduced in various human malignancies. ${ }^{19,} 20$ Until now, no metabolic actions have been assigned to DAPK. Here, we show that DAPK also binds to PKD in cardiomyocytes, but only when these cardiomyocytes are stimulated to contract. This suggests that upon contraction, DAPK binds to PKD and then activates it. Evidence for this suggestion was provided by the observation that the increase in total PKD content in DAPK immunoprecipitates upon contraction stimulation was accompanied by an increase in Ser916autophosphorylated (and hence activated) PKD in these precipitates. Silencing of DAPK further illustrates its essential role in contraction-induced PKD activation, as EFS failed to autophosphorylate PKD in DAPK-depleted HL1 cells. Furthermore, DAPK silencing abrogated contraction-induced GLUT4 translocation, thereby adding this Ser/thr kinase to the list of kinases essential for GLUT4 translocation. The in- 
volvement of DAPK in contraction-induced GLUT4 translocation is also powerful confirmation of the involvement of PKD in this translocation event. Not only PKD silencing but also silencing of its upstream kinase resulted in loss of contractioninduced GLUT4 translocation. Thus, we, demonstrate here for the first time that the tumor suppressor DAPK is involved in regulation of substrate metabolism in the heart.

With respect to contraction-induced CD36 translocation and LCFA uptake, ROS scavenging as well as DAPK silencing did not influence either one of these processes. These findings clearly illustrate that the specific involvement of PKD in GLUT4 translocation (as opposed to CD36 translocation) is reflected by its upstream kinase and second messenger. Taken together, we have unmasked a novel ROS-DAPK-PKD axis which is specifically involved in contraction-induced GLUT4 translocation and glucose uptake.

\section{Both PKD and AMPK activation are necessary for contraction-induced}

\section{GLUT4 translocation.}

One of the interesting findings in this study is that contraction-induced GLUT4 translocation is abrogated by either PKD siRNA or AMPK silencing (Fig. 1B). Hence, both kinases are necessary for this translocation event. This raises the question as to how PKD signaling can be integrated with AMPK signaling. Several lines of evidence indicate PKD and AMPK are operating in separate, independent pathways to mediate GLUT4 translocation upon contraction-stimulation. (i) We observed that PKD silencing did not affect AMPK activation nor that AMPK silencing affected PKD activation in HL1 cardiomyocytes. (ii) The ROS scavenger MPG inhibited PKD-Ser916 phosphorylation, but not AMPK-Thr172 phosphorylation in primary cardiomyocytes, indicating that ROS is implicated in PKD, but not AMPK activation. Additionally, the AMPK activating agent AICAR did not activate PKD in cardiomyocytes (unpublished observations).

This combined evidence that PKD and AMPK appear to be both necessary for GLUT4 translocation, but nonetheless contribute separately to contraction-induced signaling suggests that contraction-induced GLUT4 translocation requires the combined input of two separate contraction-activated pathways, one involving AMPK and its upstream activating kinase LKB1, ${ }^{4,6}$ the other involving PKD and its upstream kinase DAPK. These two pathways are simultaneously activated upon increased contractile activity through enhanced ATP utilization and oxidative phosphorylation, resulting in the simultaneous formation of AMP and ROS, the second messengers for these respective pathways (Fig. 5). In the present study, we have not investigated intracel- 
lular AMP levels, but earlier we observed a 2-fold increase in intracellular AMP upon $4 \mathrm{~Hz}$ contraction. ${ }^{3,6}$ This dual signaling input theory for contraction-induced GLUT4 translocation is in line with studies in skeletal muscle that demonstrated that AMPK is essential but not sufficient for contraction-induced GLUT4 translocation and glucose uptake. ${ }^{21,22}$ On the other hand, another study in skeletal muscle showed that contraction-induced GLUT4 translocation was found to be dependent on ROS signaling and independent of AMPK activation (17). Hence, further studies are needed to study the combined roles of ROS and AMPK signaling in skeletal muscle. With respect to the physiological relevance, the combined input of two separate signaling pathways may serve as a control mechanism to safeguard that under the appropriate metabolic conditions induced by contraction, i.e., simultaneous AMP formation and ROS production, GLUT4 translocation will be initiated.

\section{Figure 5}

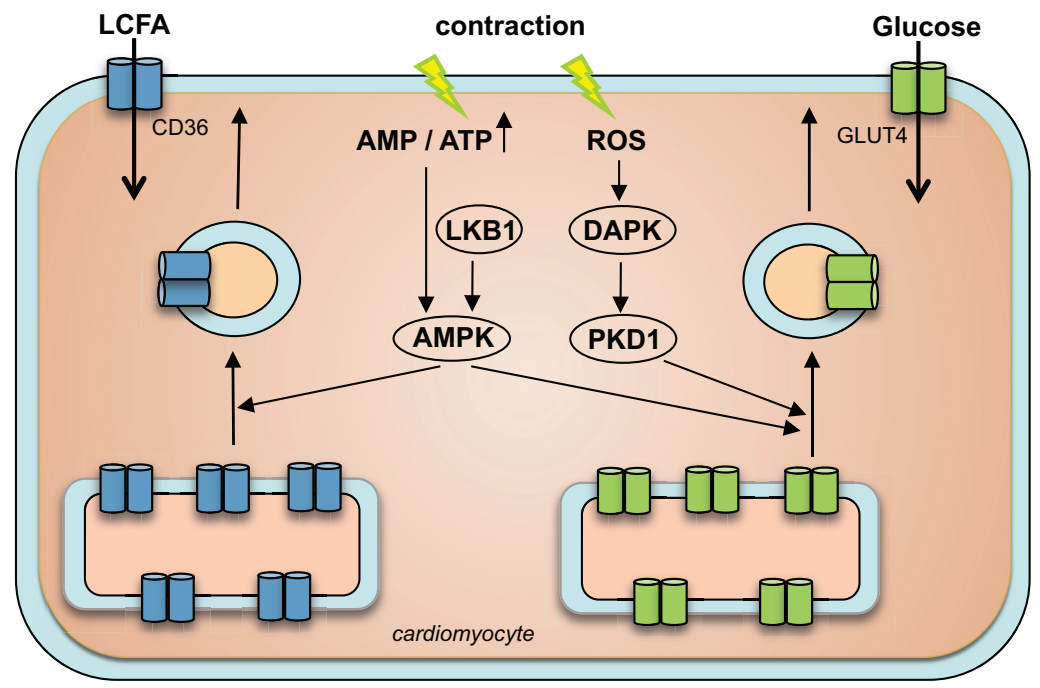

Fig. 5 Schematic presentation of the ROS-DAPK-PKD-GLUT4 axis, as a novel pathway involved in contraction-induced glucose, but not LCFA uptake. Upon contraction, both GLUT4 and CD36 are translocated from intracellular stores to the sarcolemma through a vesicle-mediated process. Whereas contractioninduced GLUT4 translocation depends on both AMPK and PKD activation, contraction-induced CD36 translocation requires AMPK, but not PKD activation. AMPK and PKD are activated upon contraction via separate signaling pathways: an increase in the AMP/ATP ratio enables LKB1 to activate AMPK; and increased ROS production and DAPK binding to PKD are upstream events for PKD activation. Remarkably, not only PKD is directly activated by a tumor suppressor (i.e., DAPK), but also LKB1, the upstream kinase of AMPK is known to be a tumor suppressor. Specifically, germline inactivation of LKB1 is involved in the development of the Peutz-Jeghers syndrome (26). Hence, there is a striking symmetry in AMPK- and PKD signaling towards GLUT4 translocation. 


\section{Future perspectives}

In conclusion, we show that in cardiomyocytes a contraction-activated ROS-DAPKPKD pathway induces GLUT4, but not CD36, translocation. Hence, specific activation of this pathway will shift cardiac substrate preference towards glucose. Increased cardiac glucose utilization has generally been linked to the development of cardiac hypertrophy via re-activation of a fetal gene program. ${ }^{23}$ In line with this, it was found that cardiospecific-PKD overexpressing mice display cardiac hypertrophy. ${ }^{24}$ The precise role of increased glucose utilization in PKD-induced cardiac hypertrophy needs further exploration. An opposite substrate preference, i.e., chronically increased LCFA utilization at the expense of glucose, is a characteristic feature of the type-2 diabetic heart, and will lead to excessive intramyocardial lipid accumulation and ultimately decreased contractile activity. ${ }^{1,25}$ Therefore, as a target for therapeutic treatment of diabetic heart disease, the ROS-DAPK-PKD pathway holds promise for the design of novel pharmaceutical compounds aimed at restoring the cardiac substrate preference via selectively inducing glucose uptake.

\section{Acknowledgements}

Financial support was from the transnational University Limburg, and the European Community (Integrated Project LSHM-CT-2004-005272, Exgenesis). The authors acknowledge the technical support of Dr. Jos Broers (Department of Cell Biology, Maastricht University) and the students Laura Schmidt and Annemarie Güntsch in performimg the microscopical experiments. 


\section{References}

1. Glatz, J.F.C ., Luiken, J.J.F.P. , and Bonen A. (2010) Physiol. Rev. 90, 367-417

2. Schwenk, R.W. , Dirkx, E. , Coumans, W.A., Bonen, A., Klip, A., Glatz, J.F.C., and Luiken, J.J.F.P. (2010) Diabetologia 53, 2209-2219

3. Luiken, J.J.F.P. , Coort, S.L.M. , Willems, J. , Coumans, W.A., Bonen, A. , van der Vusse, G.J. , and Glatz, J.F.C. (2003) Diabetes 52, 1627-1634

4. Hardie, D.G. , Hawley, S.A. , and Scott, J.W. (2006) J Physiol. 574, 7-15

5. Kurth-Kraczek, E.J. , Hirshman, M.F. , Goodyear, L.J. , and Winder, W.W. (1999) Diabetes 48, 1667-1671

6. Habets, D.D. , Coumans, W.A. , El Hasnaoui, M., Zarrinpashneh, E. , Bertrand, L. , Viollet, B. , Kiens, B. , Jensen, T.E. , Richter, E.A. , Bonen, A., Glatz, J.F.C. , and Luiken, J.J.F.P. (2009) Biochim. Biophys. Acta 1791, 212-219

7. Luiken J.J.F.P. , Vertommen, D. , Coort, S.L.M. , Habets, D.D. , El Hasnaoui, M. , Pelsers, M.M.A.L. , Viollet, B. , Bonen, A. , Hue, L. , Rider, M.H. , and Glatz J.F.C. (2008) Cell. Signal. 20, 543-556

8. Hausser, A. , Storz, P. , Märtens, S. , Link, G. , Toker, A. , and Pfizenmaier, K. (2005) Nat. Cell Biol. 7, 880-886

9. Storz, P. (2007) Trends Cell Biol. 17, 13-18

10. Luiken, J.J.F.P. , van Nieuwenhoven, F.A. , America, G. , van der Vusse, G.J., and Glatz, J.F.C. (1997) J. Lipid Res. 38, 745-758

11. Zugaza, J.L. , Sinnett-Smith, J. , Van Lint, J. , and Rozengurt, E. (1996) EMBO J. 15, 6220-6230

12. Roy, A. , Ganguly, A. , BoseDasgupta, S. , Das, B.B. , Pal, C. , Jaisankar, P. , and Majumder, H.K. (2008) Mol. Pharmacol. 74, 1292-1307

13. De Giusti, V.C. , Garciarena, C.D. , and Aiello, E.A. (2009) J. Mol. Cell. Cardiol. 47, 716-722

14. Eisenberg-Lerner, A., and Kimchi, A. (2007) Cell Death Differ. 14, 1908-1915

15. Zong, H. , Ren, J.M. , Young, .LH. , Pypaert, M. , Mu, J. , Birnbaum, M.J., and Shulman, G.I. (2002) Proc. Natl. Acad. Sci. USA 99, 15983-15987

16. Heinzel, F.R. , Luo, Y. , Dodoni, G. , Boengler, K. , Petrat, F. , Di Lisa, F. , de Groot, H. , Schulz R. , and Heusch, G. (2006) Cardiovasc. Res. 71, 374-382

17. Merry, T.L. , Steinberg, G.R. , Lynch, G.S., and McConell, G.K. (2010) Am. J. Physiol. Endocrinol. Metab. 298, E577-E585

18. Smith, M.A. , and Reid, M.B. (2006) Respir. Physiol. Neurobiol. 151, 229-241

19. Levy-Strumpf, N., and Kimchi, A. (1998) Oncogene 17, 3331-3340

20. Ng, M.H. (2002) Apoptosis 7, 261-270

21. Fujii, N. , Hirshman, M.F. , Kane, E.M. , Ho, R.C. , Peter, L.E. , Seifert, M.M. , and Goodyear, L.J. (2005) J. Biol. Chem. 280, 39033-39041

22. Lefort, N. , St-Amand, E., Morasse, S., Cote, C.H., and Marette, A. (2008) Am. J. Physiol. Endocrinol. Metab. 295, E1447-E1454

23. Taegtmeyer, H. , Sen, S. , and Vela, D. (2010) Ann. N. Y. Acad. Sci. 1188, 191-198

24. Harrison, B.C. , Kim, M.S. , van Rooij, E. , Plato, C.F. , Papst, P.J. , Vega, R.B. , McAnally, J.A. , Richardson, J.A., Bassel-Duby, R. , Olson, E.N., and McKinsey, T.A. (2006) Mol. Cell Biol. 26, 3875-3888

25. Unger, R.H. , and Orci, L. (2000) Int. J. Obes. Relat. Metab. Disord. 24 Suppl. 4, S28-S32.

26. Hemminki, A. , Markie, D. , Tomlinson, I. , Avizienyte, E. , Roth, S, Loukola, A, Bignell, G. , Warren, W. , Aminoff, M. , Höglund, P, Järvinen, H. , Kristo, P. , Pelin, K, Ridanpää, M. , Salovaara, R. , Toro, T. , Bodmer, W. , Olschwang, S. , Olsen, A.S. , Stratton, M.R. , de la Chapelle, A. , and Aaltonen, L.A. (1998) Nature 391, 184-187 
CHAPTER 4

\section{Detection of cardiac myosin binding protein-C, by a phospho-specific PKD an- tibody in contracting rat cardiomyocytes}

Ellen Dirkx ${ }^{a}$, Freek G. Bouwman ${ }^{c}$, Didier Vertommen ${ }^{b}$, Edwin C. Mariman ${ }^{c}$, Sakthivel Sadayappan $^{\text {d }}$, Jan F.C. Glatz ${ }^{a}$, Joost J.F.P. Luiken ${ }^{a}$, Guillaume J.J.M. van Eys ${ }^{a}$

\footnotetext{
${ }^{a}$ Department of Molecular Genetics, Cardiovascular Research Institute Maastricht, Maastricht University, the Netherlands

${ }^{\mathrm{b} H O R M}$-PHOS Unit, Université Catholique de Louvain (UCL), and de Duve Institute, Brussels, Belgium.

'Department of Human Biology, Nutrition and Toxicology Research Institute Maastricht (NUTRIM), Maastricht University, the Netherlands.

${ }^{d}$ Department of Cell and Molecular physiology, Stritch School of Medicine, Loyola University Chicago, Illinois
} 


\begin{abstract}
Protein phosphorylation plays an important role in physiological processes. Phospho-specific antibodies have become powerful tools to study processes such as muscle contraction. Cardiac myosin binding protein-C (cMyBP-C) is one of the proteins that make up the contractile apparatus of cardiomyocytes. Phosphorylation of CMyBP-C is essential for normal cardiac function, since dephosphorylation of this protein leads to degradation and has been associated with cardiomyopathy. One of the upstream kinases, which phosphorylate cMyBP-C, is protein kinase $D$ (PKD). While studying the role of PKD in CMyBP-C phosphorylation, we tried to analyze phosphorylation of PKD with a phospho-specific PKD-Ser744/748 antibody. Contrary to the expected $115 \mathrm{kDa}$, a signal was found for a $150 \mathrm{kDa}$ protein. By MALDI-TOF mass spectrometry, we identified this protein to be cMyBP-C. These data were confirmed by immunostainings using the p-PKDSer744/748 antibody, which displayed a striated pattern similar to the one observed for a regular CMyBP-C antibody. To our knowledge there are no antibodies commercially available for phosphorylated cMyBP-C. Thus, the p-PKD-Ser744/748 antibody can accelerate research into the role of cMyBP-C phosphorylation in cardiomyocytes.
\end{abstract}




\section{Introduction}

Reversible phosphorylation changes kinetic properties of proteins. By phosphorylation protein kinases can modify the function of a protein in almost every conceivable way. For instance, kinases influence enzymatic activities, movement between subcellular compartments, and protein-protein interactions. ${ }^{1}$ Therefore, protein phosphorylation is now recognized to play a critical role in many physiological processes. ${ }^{1}$ Thus, aberrant phosphorylation will disrupt these processes and is expected to be the cause of a number of human diseases. As a result, it has become increasingly important to determine changes in the phosphorylation state of proteins. Phospho-specific antibodies are important and powerful tools to study these processes, because they can detect phosphorylation of a specific site or motif of a protein.

Heart failure in mouse models and in humans has been associated with dephosphorylation and subsequent degradation of cardiac myosin binding protein-C (cMyBP-C). ${ }^{2-4}$ The decrease in CMyBP-C interferes with cross-bridge formation that has been demonstrated to depend on cMyBP-C phosphorylation through enzymes such as $\mathrm{Ca}^{2+}$-calmodulin-activated kinase II, protein kinase $\mathrm{A}$ and protein kinase $\mathrm{C}^{5}$ Also, mutations in this protein, possibly leading to an accelerated degradation of cMyBP-C, have been linked to cardiomyopathy. ${ }^{5,6}$ cMyBP-C is thought to facilitate interaction between myosin and actin by tethering both molecules but the exact mechanism is hardly understood. The elucidation of the precise function of CMyBP-C phosphorylation is also clinically important because CMyBP-C phosphorylation has been suggested to be useful as a biomarker for diagnosing myocardial infarction and as a potential target for therapeutic intervention. ${ }^{5,6}$ However, such medical applications depend on insight in the role of CMyBP-C phosphorylation in cardiac contraction.

Protein kinase D1 (PKD1) has been shown to participate in many signaling cascades. The 115 kDa PKD1 belongs to a serine/threonine kinase family and contains an $\mathrm{N}$-terminal regulatory domain and a C-terminal kinase domain (Fig 1$)^{7}$ Its activity, phosphorylation of downstream targets in the signaling cascade, depends on its phosphorylation state as a number of residues can be (de)phosphorylated, giving rise to specific reaction profiles. In the heart it has been demonstrated to affect contractility, metabolism and remodeling. ${ }^{5,-10}$ Recently, PKD (in addition to the above mentioned kinases) has been demonstrated to phosphorylate cMyBPC. ${ }^{10}$ However, the relevance of this CMyBP-C modification is still a matter of research, which has been hampered by the lack of adequate tools to detect phosphorylated cMyBP-C.

During a series of experiments aimed at determining the role of PKD in CMyBP-C phosphorylation we observed an aberrant band in western blots probed with a $\mathrm{p}$ PKD-Ser744-748 antibody. Further investigations showed that this PPKD "specific" antibody recognized pcMyBP-C, making this antibody an important tool in further research into the function of $\mathrm{CMyBP}-\mathrm{C}$. 


\section{Materials and Methods}

\section{Materials}

Antibodies directed against phospho-PKD/PKC $-\mu$ (Ser916), phospho-PKD/PKC- $\mu$ (Ser744/748), and caveolin-3 were obtained from Cell Signaling (Beverly, USA). The MYBPC3 (cMyBP-C) antibody was obtained from Santa Cruz Biotechnology, Inc. (Santa Cruz, USA). The phospho-specific cMyBP-C-Ser315 antibody was house-made by Dr. Sadayappan. ${ }^{11}$ The Titin antibody (mouse monoclonal, T12) was a gift from Prof. Furst, (Potsdam, Germany) and the Desmin antibody (rabbit polyvalent) was a gift from Dr. Schaart, (Maastricht, the Netherlands). Secondary antibodies used for the immunostaining (goat-anti-mouse, goat-anti-rabbit, and rabbit anti-goat) were purchased from DAKO (Denmark). Oligomycin and phenylephrine were purchased from Sigma-Aldrich (St. Louis, USA).

\section{Isolation and treatment of adult rat cardiomyocytes}

Cardiomyocytes were isolated from male Lewis rats (200-250 g) (obtained from Charles River) using a Langendorff perfusion system and a Krebs Henseleit bicarbonate medium equilibrated with a $95 \% \mathrm{O}_{2} / 5 \% \mathrm{CO}_{2}$ gas phase at $37^{\circ} \mathrm{C}$ as previously described. ${ }^{12}$ Cardiomyocytes were incubated for $15 \mathrm{~min}$ at $37^{\circ} \mathrm{C}$ with continuous shaking either with $5 \mu \mathrm{mol} / \mathrm{L}$ oligomycin, $50 \mu \mathrm{mol} / \mathrm{L}$ phenylephrine or electrically stimulated for 6 minutes, using a commercially available IONOPTIX stimulator $(4 \mathrm{~Hz}$, $40 \mathrm{~V}$, pulse duration $5 \mathrm{~ms}$ ).

\section{Immunoblotting}

Proteins were separated by SDS-PAGE and transferred to nitrocellulose membranes for Western Blotting. The membranes were probed with the relevant primary antibodies. The protein bands were visualized using enhanced chemiluminescence and immunoblot intensities were analyzed by densitometry. ${ }^{8}$

\section{D-gel-electrophoresis}

Total protein $(50 \mu \mathrm{g})$ was loaded for the first dimension. Iso-electric focusing (IEF) was preformed on an IPG PHOR electrophoresis unit (Amersham Biosciences) at 20 ․ C. IEF was performed using the following program: $500 \mathrm{~V}$ for $1 \mathrm{~h}, 1000 \mathrm{~V}$ for $1 \mathrm{~h}$, $1000-8000 \mathrm{~V}$ for $2 \mathrm{~h}$ and a final step of $8000 \mathrm{~V}$ for $56250 \mathrm{Vh}$. The second-dimension run was carried out on $12.5 \%$ SDS-PAGE gels. Electrophoresis was conducted at a constant voltage of $200 \mathrm{~V}$ for $5 \mathrm{~h}$ in a $24 \mathrm{~cm}$ Dodeca Cell (Bio-Rad, Veenendaal, the 
Netherlands). ${ }^{13}$ Proteins were stained with Sypro Ruby. Gel images were obtained with a FX Molecular Imager (Bio-Rad, Veenendaal, the Netherlands).

\section{In-gel digestion}

Protein spots were excised from gels using an automated spot cutter (Bio-Rad, Veenendaal, the Netherlands) and processed on a MassPREP digestion robot (Waters, Manchester UK). A solution of $50 \mathrm{mmol} / \mathrm{L}$ ammonium bicarbonate in $50 \%(\mathrm{v} / \mathrm{v})$ acetonitrile was used for destaining. Cysteines were reduced with $10 \mathrm{mmol} / \mathrm{L}$ DTT in $100 \mathrm{mmol} / \mathrm{L} \mathrm{NH}_{4} \mathrm{CO}_{3}$ for $30 \mathrm{~min}$ followed by alkylation with $55 \mathrm{mmol} / \mathrm{L}$ iodoacetamide in $100 \mathrm{mmol} / \mathrm{L} \mathrm{NH}_{4} \mathrm{CO}_{3}$ for $20 \mathrm{~min}$. Spots were washed with $100 \mathrm{mmol} / \mathrm{L} \mathrm{NH}_{4} \mathrm{CO}_{3}$ to remove excess reagents and were subsequently dehydrated with $100 \%$ acetonitrile. Trypsin $(6 \mathrm{ng} / \mu \mathrm{l})$ in $50 \mathrm{mmol} / \mathrm{L} \mathrm{NH}_{4} \mathrm{CO}_{3}$ was added to the gel plug and incubation was preformed at $37{ }^{\circ} \mathrm{C}$ for $5 \mathrm{~h}$. Peptides were extracted in $30 \mu \mathrm{l}$ of $1 \%(\mathrm{v} / \mathrm{v})$ formic acid / $2 \%(\mathrm{v} / \mathrm{v})$ acetonitrile in water for $30 \mathrm{~min}$ at room temperature. A second extraction was performed using $24 \mu \mathrm{l}$ of $50 \%(\mathrm{v} / \mathrm{v})$ acetonitrile in water for 20 $\min$ at room temperature. ${ }^{13}$

\section{Mass spectrometry}

For MALDI-TOF mass spectrometry $1.5 \mu \mathrm{l}$ of peptide mixture and $0.5 \mu \mathrm{l}$ matrix solution $(2.5 \mathrm{mg} / \mathrm{ml} \alpha$-cyano-4-hydroxycinnamic acid in $50 \%$ acetonitrile / $0.1 \%$ TFA) was spotted automatically onto a 96 well-format target plate. Spots were allowed to air dry for homogeneous crystallization. Spectra were obtained using a MALDI-LR mass spectrometer (Waters). The instrument was operated in positive reflector mode. Acquisition mass range was 800-4.000 Da. The instrument was calibrated on 10-12 reference masses from a tryptic digest of alcohol dehydrogenase. In addition, a near point lockmass correction for each sample spot was performed using adrenocorticotropic hormone fragment $18-39(\mathrm{MH}+2465.199)$ to achieve maximum mass accuracy. Typically 120 shots were combined and background subtracted. A peptide mass list was generated for the subsequent database search. ${ }^{13}$

\section{Database search}

The peptide mass list was searched with the Mascot search engine (http://www.matrixscience.com) against the Swiss-Prot database (http://expasy.ch/sprot) for protein identification. One missed cleavage was tolerated; carbamidomethylation was set as a fixed modification and oxidation of methionine as an optional modification. The peptide mass tolerance was set to $100 \mathrm{ppm}$. No restrictions were made on the protein molecular mass and the isoelectric point. 
A protein was regarded identified when it had a significant Mascot probability score $(p<0.05)$. $^{13}$

\section{Immunostaining}

Isolated rat cardiomycytes were immunostained with several antibodies. To determine the integrity status of the cells antibodies against desmin and titin were applied. Cells were fixed by a short immersion in ice-cold methanol. After air-drying the cells were incubated for $1 \mathrm{~h}$ at RT with $1 / 20$ horse serum. After a short rinse primary antibodies were applied (Titin 1/10, Desmine $1 / 25$, cMyBP-C $1 / 10$ and pPKD-Ser744/748 1/20) and the cells were incubated overnight at $4^{\circ} \mathrm{C}$. After washing the cells in PBS for 2 times 10 min, they were again blocked with 1/20 horse serum and after a short rinse in PBS incubated with the second antibodies (goat-antimouse, goat-anti-rabbit, and rabbit anti-goat) (1/1000 dilution) for $1 \mathrm{~h}$ at RT. The second antibodies were labeled with FITC ( $G a R, R a G)$. Then the cells were washed in PBS for $30 \mathrm{~min}$ with several changes of washing buffer. Finally, the cells were mounted in $50 \%$ glycerol containing DAPI.

\section{Results and Discussion}

Stimulation of rat cardiomyocytes by contraction (electrical field stimulation (EFS)), oligomycin (oli) or phenylephrine (PE) resulted in PKD-Ser916 phosphorylation (PKD visible as a $115 \mathrm{kDa}$ band) (Figure 1). Probing of the same blots with a phosphospecific PKD-Ser744/748 antibody, showed that only phenylephrine was able to phosphorylate the PKD-Ser744/748 site (at $115 \mathrm{kDa}$ ) (Figure 1). This is in agreement with previous observations which showed that contraction/oligomycin-treatment of cardiomyocytes did not result in translocation of various $\mathrm{PKCs}{ }^{8}$ and showed that this type of PKD activation is independent of Ser744/747 phosphorylation. However, using the phospho-specific PKD-Ser744/748 antibody, a signal of approximately 150 $\mathrm{kDa}$ was detected in electrical field stimulated, as well as oligomycin or phenylephrine treated cardiomyocytes (Figure 1). 


\section{Figure 1}
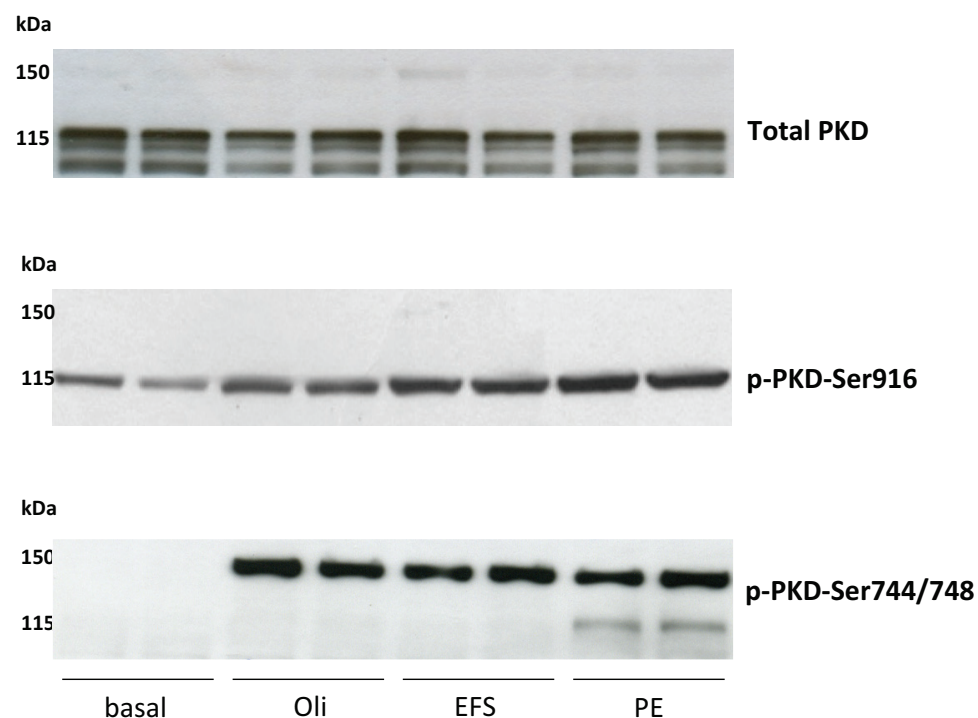

Fig. 1 Phosphorylation of PKD upon different stimuli. Isolated cardiomyocytes were treated with $5 \mu \mathrm{mol} / \mathrm{L}$ oligomycin (oli) or $50 \mu \mathrm{mol} / \mathrm{L}$ phenylephrine (PE) for $15 \mathrm{~min}$, or electrically field stimulated (EFS) and were compared to non-stimulated cells. Samples were immunoblotted against total PKD protein expression (115 kDa), p-PKD-Ser916 (115 kDa), p-PKD-Ser744/748 (150 kDa).

This surprising observation suggested that the phospho-PKD Ser744/748 antibody does not detect phosphorylation of PKD (115 kDa), but rather phosphorylation of another protein with a molecular mass of $150 \mathrm{kDa}$. To identify the $150 \mathrm{kDa}$ protein band, detected by the phospho-PKD-Ser744/748 antibody, lysates from control and oligomycin-treated cardiomyocytes were subjected to 2D-gel-electrophoresis, followed by analysis with the phospho-PKD-Ser744/748 antibody. First, lysates dissolved in ureum buffer from control and oligomycin-treated cardiomyocytes were western blotted against p-PKD-Ser744/748 to confirm PKD-Ser744/748 phosphorylation (PE treatment was used as a control) (Figure 2A). Next, total protein $(50 \mu \mathrm{g})$ was loaded from oligomycin-treated cardiomyocytes for the 2D-gel electrophoresis. The protein spots appearing after Sypro Ruby staining (Figure 2B) were compared with the protein pattern from the immunoblotting (Figure $2 \mathrm{C}$ ), and the matching spots were excised from the gel. Further analysis was done with a MALDI-TOF mass spectrometer. The isolated protein was identified as CMyBP-C with a mascot score of 170 for the SDS-PAGE gel and a mascot score of 107 for the 2D gel. Furthermore, we showed that, after CMyBP-C immunoprecipitation of oligomycin-stimulated cardiomyocytes, the p-PKD-Ser744/748 antibody detected selectively cMyBP-C phosphorylation (150 kDa) and not PKD phosphorylation (115 $\mathrm{kDa}$ ) (Figure 2D). To verify these data, immunostainings for cMyBP-C with the $\mathrm{p}$ PKD-Ser744/748 antibody were performed. As a control, staining for titin and desmin were used, which clearly reveals the striated structure of the cardiomycytes 
and shows the structural integrity of the cells. The cMyBP-C antibody (from Santa Cruz), as expected, reveals also a striated pattern (Figure 2E). The striation is not as sharp as for desmin and titin, which may be due to the fact that not all cMyBP-C proteins are attached to the contractile apparatus. The p-PKD-Ser315/320 antibody also provides a striated picture, much like the cMyBP-C antibody (Figure 2E). Other PKD antibodies give a diffuse pattern (data not shown). Thus, these results demonstrate that the p-PKD-Ser744/748 antibody is able to detect cMyBP-C in isolated rat cardiomyocytes.

\section{Figure 2}

A

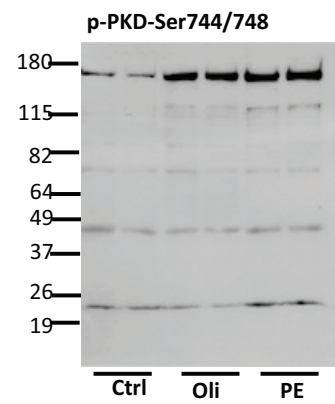

B Sypro Ruby stain

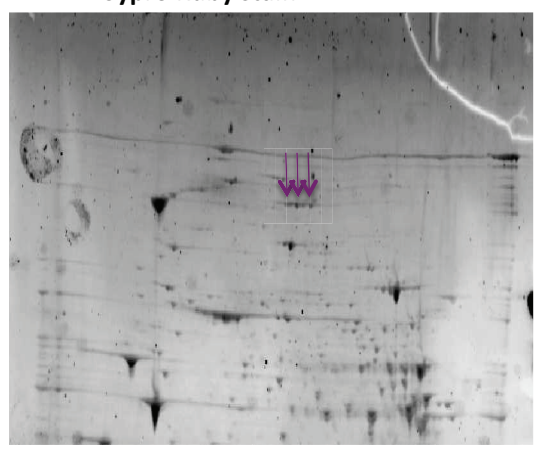

$\mathrm{D}$
C Western blot: p-PKD-Ser744/748

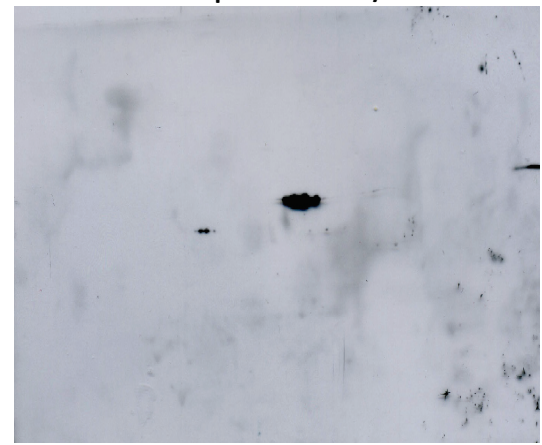

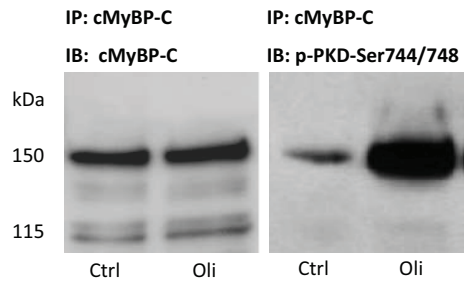




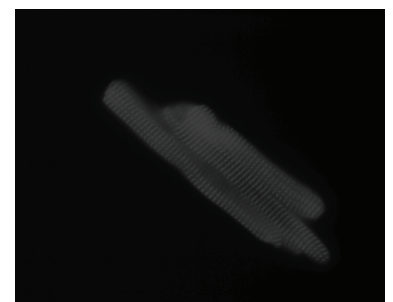

Titin

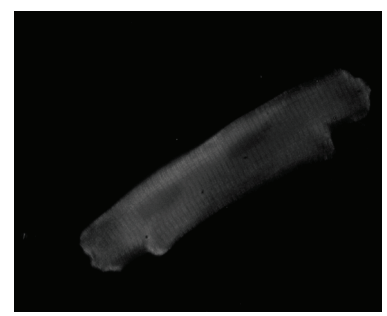

cMyBP-C

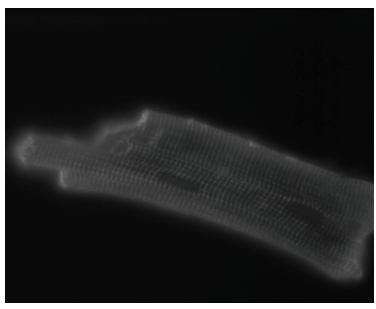

Desmin

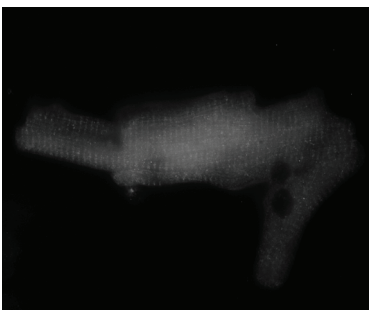

p-PKD-Ser744/748

Fig. 2 Dectection of $C M y B P-C$ with the $p-P K D-S e r 744 / 748$ antibody. Isolated rat cardiomyocytes were treated with $5 \mu \mathrm{mol} / \mathrm{L}$ oligomycin for $15 \mathrm{~min}$ at $37 \circ \mathrm{C}$. Incubations were terminated by centrifugation and subsequent suspension of the cells in urea buffer or sample buffer. (A) Western blotting was performed to confirm the phosphorylation pattern with the p-PKD-Ser744/748 antibody (phenylephrine stimulation was used as a control). (B) $50 \mu \mathrm{g}$ of protein was separated by SDS-PAGE. Afterwards the gel was stained with Sypro Ruby stain to visualize all protein spots. (C) The protein spots appearing after immunoblotting, using the phospho-PKD-Ser744/48 antibody, were excised on a glass plate from the Sypro Ruby stained gel (the excised spots were indicated with arrows) and processed on a MassPREP digestion robot. Further analysis was done with a MALDI-TOF mass spectrometer (D) Isolated cardiomyocytes were treated with 5 $\mu \mathrm{mol} / \mathrm{L}$ oligomycin (oli) whereafter CMyBP-C was immunoprecipitated. Samples were immunoblotted against CMyBP-C and p-PKD-Ser744/748. (E) immunostaining was performed with antibodies against Titin, Desmin, cMyBP-C and p-PKD-Ser744/748.

Knowing that the phospho-PKD-Ser744/748 antibody recognizes a short sequence of amino acids within the activation loop of PKD containing both Ser744 and Ser748 residues, we reasoned that a loop with a similar amino acid sequence could be expected to be present within CMyBP-C. Indeed, cMyBP-C possesses a phosphomotif around Ser315/320 with strong homology to the Ser744/748 site of PKD (Figure $3 \mathrm{~A}$ ). To verify this, we used a home-made phospho-specific cMyBP-C antibody. ${ }^{11}$ Also with this antibody we detected phosphorylation of cMyBP-C-Ser315 during contraction (Figure 3B This finding is in agreement with previous data, which showed that the region around Ser315 in the rat, that is similar to the region around Ser302 in the mouse, is a phosphorylation site of cMyBP-C. ${ }^{5,14}$ Thus, the p-PKDSer744/748 antibody recognizes this sequence with considerable homology between PKD and CMyBP-C if both serine residues are phosphorylated. 


\section{Figure 3}

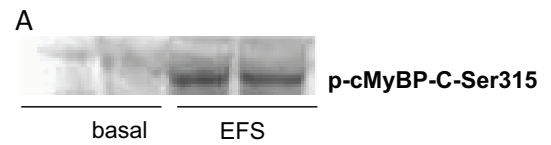

B

PKD-Ser744/748 riigeksfrrsvvgtpayl

$744 \quad 748$

cMyBP-C-Ser315-320 llkkrd\$frrdskleapae

315320

Fig. 3 The $p$-PKD-Ser744/748 antibody recognizes phosphor-region around cMyBP-C-Ser315/320. (A) Comparison of the PKD-Ser744/748 sequence with Ser315/320 of cMyBP-C in rat cardiomyocytes. (B) Isolated cardiomyocytes were electric field stimulated (EFS). Samples were blotted with a phosphospecific cMyBP-C-Ser315 antibody.

\section{Conclusion}

In this study we describe the finding that a phospho-specific antibody directed against PKD-Ser744/748, can be used to detect phosphorylation of cMyBP-C in contracting cardiomyocytes. So far, there are no phospho-cMyBP-C antibodies commercially available. Although, this p-PKD-Ser744/748 antibody was not designed to detect $\mathrm{CMyBP-C}$, it can contribute in defining the role of cMyBP-C phosphorylation and more in general the function of $\mathrm{CMyBP}-\mathrm{C}$. Since phosphorylation of $\mathrm{CMyBP}-\mathrm{C}$ is markedly decreased in human heart failure and experimental models, ${ }^{5,15,16}$ this may open new ways for early diagnosis and preventive treatment of cardiac pathologies.

\section{Acknowledgements}

This study was funded by the transnational University Limburg (tUL) and the European Community (Integrated Project LSHM-CT-2004-005272, Exgenesis). DV is "Collaborateur Logisitique" of the FRS-FNRS (Belgium)

\section{Disclosures}

none 


\section{References}

1. Cohen P. The origins of protein phosphorylation. Nat Cell Biol. 2002;4:E127-130

2. Decker RS, Decker ML, Kulikovskaya I, Nakamura S, Lee DC, Harris K, Klocke FJ, Winegrad S. Myosin-binding protein c phosphorylation, myofibril structure, and contractile function during low-flow ischemia. Circulation. 2005;111:906-912

3. Jacques AM, Copeland O, Messer AE, Gallon CE, King K, McKenna WJ, Tsang VT, Marston SB. Myosin binding protein $\mathrm{c}$ phosphorylation in normal, hypertrophic and failing human heart muscle. Journal of molecular and cellular cardiology. 2008;45:209-216

4. Sadayappan S, Osinska H, Klevitsky R, Lorenz JN, Sargent M, Molkentin JD, Seidman CE, Seidman JG, Robbins J. Cardiac myosin binding protein c phosphorylation is cardioprotective. Proceedings of the National Academy of Sciences of the United States of America. 2006;103:16918-16923

5. Barefield D, Sadayappan S. Phosphorylation and function of cardiac myosin binding protein-c in health and disease. J Mol Cell Cardiol. 2010;48:866-875

6. Schlossarek S, Mearini G, Carrier L. Cardiac myosin-binding protein c in hypertrophic cardiomyopathy: Mechanisms and therapeutic opportunities. J Mol Cell Cardiol. 2011;50:613620

7. Rykx A, De Kimpe L, Mikhalap S, Vantus T, Seufferlein T, Vandenheede JR, Van Lint J. Protein kinase d: A family affair. FEBS Lett. 2003;546:81-86

8. Luiken JJ, Vertommen D, Coort SL, Habets DD, El Hasnaoui M, Pelsers MM, Viollet B, Bonen A, Hue L, Rider $\mathrm{MH}$, Glatz JF. Identification of protein kinase $d$ as a novel contraction-activated kinase linked to glut4-mediated glucose uptake, independent of ampk. Cell Signal. 2008;20:543-556

9. Fielitz J, Kim MS, Shelton JM, Qi X, Hill JA, Richardson JA, Bassel-Duby R, Olson EN. Requirement of protein kinase d1 for pathological cardiac remodeling. Proc Natl Acad Sci U S A. 2008;105:3059-3063

10. Bardswell SC, Cuello F, Rowland AJ, Sadayappan S, Robbins J, Gautel M, Walker JW, Kentish JC, Avkiran M. Distinct sarcomeric substrates are responsible for protein kinase d-mediated regulation of cardiac myofilament ca2+ sensitivity and cross-bridge cycling. J Biol Chem. 2010;285:5674-5682

11. Sadayappan S, Osinska H, Klevitsky R, Lorenz JN, Sargent M, Molkentin JD, Seidman CE, Seidman JG, Robbins J. Cardiac myosin binding protein c phosphorylation is cardioprotective. Proc Natl Acad Sci U S A. 2006;103:16918-16923

12. Luiken JJ, van Nieuwenhoven FA, America G, van der Vusse GJ, Glatz JF. Uptake and metabolism of palmitate by isolated cardiac myocytes from adult rats: Involvement of sarcolemmal proteins. J Lipid Res. 1997;38:745-758

13. Bouwman F, Renes J, Mariman E. A combination of protein profiling and isotopomer analysis using matrix-assisted laser desorption/ionization-time of flight mass spectrometry reveals an active metabolism of the extracellular matrix of 3t3-I1 adipocytes. Proteomics. 2004;4:38553863

14. Wu SC, Solaro RJ. Protein kinase c zeta. A novel regulator of both phosphorylation and dephosphorylation of cardiac sarcomeric proteins. J Biol Chem. 2007;282:30691-30698

15. El-Armouche A, Pohlmann L, Schlossarek S, Starbatty J, Yeh YH, Nattel S, Dobrev D, Eschenhagen T, Carrier L. Decreased phosphorylation levels of cardiac myosin-binding proteinc in human and experimental heart failure. J Mol Cell Cardiol. 2007;43:223-229

16. Sadayappan S, Gulick J, Osinska H, Martin LA, Hahn HS, Dorn GW, 2nd, Klevitsky R, Seidman CE, Seidman JG, Robbins J. Cardiac myosin-binding protein-c phosphorylation and cardiac function. Circ Res. 2005;97:1156-1163 


\section{CHAPTER 5}

\section{Protein kinase $\mathrm{D}$ increases maximal $\mathrm{Ca}^{2+}$ - activated tension of cardiomyocyte con- traction by selective phosphorylation of cMyBP-C-Ser315}

Ellen Dirkx ${ }^{a}$, Olivier Cazorla ${ }^{b}$, Robert W. Schwenk ${ }^{a}$, Ilka Lorenzen-Schmidt ${ }^{c}$, Sakthivel Sadayappan ${ }^{d}$, Johan Van Lint ${ }^{e}$, Lucie Carrier ${ }^{f}$, Guillaume J.J.M. van Eys ${ }^{\text {a }}$, Jan F.C. Glatz ${ }^{a}$ and Joost J.F.P. Luiken ${ }^{a}$

\footnotetext{
a'Department of Molecular Genetics, Cardiovascular Research Institute Maastricht, Maastricht University, the Netherlands. ${ }^{b}$ Inserm U637, Université Montpellier1, F-34295, Montpellier, France 'Department of Physiology, Cardiovascular Research Institute Maastricht, Maastricht University, the Netherlands. ${ }^{d}$ Department of Cell and Molecular Physiology, Loyola University Chicago, USA, ${ }^{\mathrm{e}} \mathrm{HORM}$-PHOS Unit, Université Catholique de Louvain (UCL), Leuven, Belgium. 'Department of Human Biology, Nutrition and Toxicology Research Institute Maastricht (NUTRIM), Maastricht University, the Netherlands. ${ }^{\mathrm{g}}$ Dept of Molecular Cell Biology - Faculty of Medicine, Katholieke Universiteit Leuven (KUL), Belgium. ' Department of Experimental and Clinical Pharmacology and Toxicology, Cardiovascular Research Center, University Medical Center Hamburg-Eppendorf, Germany. Inserm, U974, Institut de Myologie, Paris, F-75013, France ${ }^{f}$ Université Pierre et Marie Curie-Paris6, UMR-S974, CNRS, UMR7215, Institut de Myologie, IFR14, Paris, F75013 ,France
} 


\section{ABSTRACT}

Cardiac myosin binding protein $\mathrm{C}$ ( $\mathrm{CMyBP}-\mathrm{C})$ is involved in the regulation of cardiac myofilament contraction. Recent evidence showed that Protein kinase D (PKD) is one of the kinases that phosphorylates cMyBP-C. However, the mechanism by which PKD-induced CMyBP-C phosphorylation mediates cardiac contractile responses is not known. Using immunoprecipitation, we showed that in contracting cardiomyocytes, PKD binds to cMyBP-C and phosphorylate it at Ser315. The effect of PKDmediated phosphorylation of $\mathrm{CMyBP-C}$ on cardiac myofilament function was investigated in permeabilized ventricular myocytes isolated from wild-type (WT) and from cMyBP-C knock-out (KO) mice, incubated in the presence of constitutively active PKD. In WT myocytes, PKD increased both myofilament $\mathrm{Ca}^{2+}$ sensitivity $\left(\mathrm{pCa}_{50}\right)$ and maximal $\mathrm{Ca}^{2+}$-activated tension of contraction (Tmax). In CMyBP-C KO myofilaments, PKD also increased pCa50, but failed to alter Tmax. This indicates that cMyBP-C is not involved in PKD-mediated sensitization of myofilaments to $\mathrm{Ca} 2+$, but is essential for PKD-induced increase of Tmax. Furthermore, the phosphorylation of both PKD-Ser916 and CMyBP-C-Ser315 was contraction frequency-dependent, indicating that PKD-mediated cMyBP-C phosphorylation is operational especially during periods of increased contractile activity. Thus, during increased workload, PKD1 facilitates contraction of the cardiomyocytes by increasing $\mathrm{pCa} 50$ and by phosphorylating cMyBP-C to increase Tmax. 


\section{Introduction}

Protein kinase $D(P K D)$ belongs to a serine/threonine kinase family that is closely related to novel and conventional members of the protein kinase $C$ (PKC) family, with which it shares the ability to be activated by diacylglycerol (DAG). ${ }^{1}$ One of the mechanisms to activate PKD, is via an A-kinase anchoring protein, AKAP-Lbc, which assembles a PKD activation complex and targets PKD to a specific subcellular location. ${ }^{2}$ AKAP-Lbc then recruits an upstream kinase, PKC, and coordinates protein kinase $A$ (PKA) phosphorylation events that lead to the release of activated PKD at a specific location into the cell. Thus, AKAP-Lbc synchronizes PKA and PKC activities in a manner that leads to the activation of PKD. ${ }^{2}$

Several proteins have been identified to be substrates of PKD, including Troponin I $(T n l)$, telethonin, and cardiac myosin binding protein $C$ (cMyBP-C). ${ }^{3}$ cMyBP-C is a component of the cardiac sarcomere thick filaments that contributes to myosin assembly. ${ }^{4}$ CMyBP-C prevents weakly bound cross-bridges and increases force during the power stroke, thereby limiting ATP consumption per cross-bridge force production cycle. ${ }^{5}$ Furthermore, cMyBP-C has been shown to play a role in maintaining complete relaxation of cardiomyocytes. ${ }^{6}$ Several animal models have been developed to evaluate the role of cMyBP-C. Most of the introduced mutations in the CMyBP-C gene resulted in hypertrophic cardiomyopathy, indicative for a reduced contractile power. ${ }^{4,7}$ In the rat, three phosphorylation sites in the MyBP-C motif located between C1 and C2 domains have been identified, including Ser287, Ser295, and Ser315. ${ }^{9,10}$ In mouse myocardium the cMyBP-C-Ser302 is structurally identical to the Ser315 phosphorylation-site in rat. Upon phosphorylation of the MyBP-C motif, cMyBP-C interacts with the S2 region of myosin at its C1-C2-MyBP-C domains. ${ }^{4}$ cMyBP-C also binds to actin at the C1-MyBP-C motif, which could be important for the arrangement of myosin-actin filaments in the sarcomere. ${ }^{4}$ Phosphorylation of CMyBP-C by PKA appears to accelerate the rates of force development in systole and the rates of relaxation in diastole, thereby increasing cardiac contractility. ${ }^{11,12}$ Recently, in connection with an observed binding of PKD to CMyBP-C in quiescent trabeculae, Bardswell and coworkers demonstrated that the catalytic subunit of PKD interacts with cMyBP-C-Ser302 in skinned mouse myocardium. ${ }^{4}$ Independently, PKD has been shown to be activated during contraction of cardiomyocytes. ${ }^{13}$ Whether PKD-mediated cMyBP-C phosphorylation is also increased upon contraction of cardiomyocytes remains to be established.

Phosphorylation of CMyBP-C is significantly reduced in patients with heart failure as it is in experimental models mimicking this pathology. ${ }^{14,15}$ Therefore, it is essential to study the physiological relevance of PKD-induced cMyBP-C phosphorylation during contraction, which is the natural state of living cardiomyocytes. In the present study we analyzed the consequences of increased workload on PKD-mediated CMyBP-C phosphorylation, and how this phosphorylation facilitates coping with increased contractile demands. 


\section{Materials and Methods}

\section{Animals}

Homozygous cardiac cMyBP-C-null (KO) mice were generated in a Black Swiss background as previously described by Carrier et al. ${ }^{16}$ Male Lewis rats (200-250 g) were obtained from Charles River.

\section{Materials}

Antibodies directed against PKD/PKC $\mu$, phospho-PKD/PKC- $\mu$ (Ser916) were obtained from Cell Signaling (Beverly, USA). The MYBPC3 antibody was obtained from Santa Cruz Biotechnology, Inc. (Santa Cruz, USA) and the rat cMyBP-Cser315 antibody (which is identical to CMyBP-C-Ser302 in mouse was home-made by Sadayappan. ${ }^{17}$ The Full length active PKD was home-made by the laboratory of Prof. Johan Van Lint. ${ }^{18}$ ). All culture dishes, pipets, tubes and containers used, were from Greiner Bio-One (Alphen aan de Rijn, the Netherlands).

\section{Isolation and treatment of adult rat cardiomyocytes}

Cardiomyocytes were isolated from male Lewis rats (200-250 g) using a Langendorff perfusion system and a Krebs Henseleit bicarbonate medium equilibrated with a $95 \% \mathrm{O}_{2} / 5 \% \mathrm{CO}_{2}$ gas phase at $37^{\circ} \mathrm{C}$ as previously described ${ }^{19}$. Cardiomyocytes were incubated for $6 \mathrm{~min}$ at $37^{\circ} \mathrm{C}$ with continuous shaking and electrically stimulated for 6 minutes, using a commercially available IONOPTIX stimulator (4 $\mathrm{Hz}, 40 \mathrm{~V}$, pulse duration $5 \mathrm{~ms}$ ).

\section{Immunoprecipitation}

Cells were pelleted and resuspended for $1 \mathrm{~h}$ in ice-cold lysis buffer: $50 \mathrm{mmol} / \mathrm{L}$ Tris- $\mathrm{HCl}(\mathrm{pH}=7.4), 150 \mathrm{mmol} / \mathrm{L} \mathrm{NaCl}, 1 \%$ IGEPAL CA-630, 0.5\% sodium deoxycholate, 0.1\% SDS, Complete protease inhibitor (Roche) and PhosSTOP phosphatase inhibitor (Roche). Supernatant fractions $(500 \mu \mathrm{l})$ were incubated with the PKD or MYBPC3 (K-16) antibody $(5 \mu \mathrm{g})$ overnight at $4^{\circ} \mathrm{C}$ on a rotating wheel. Thereafter, $50 \mu \mathrm{l}$ of Protein G Sepharose bead suspension (GE Healthcare) was added and incubations were continued for $1 \mathrm{~h}$ at $4^{\circ} \mathrm{C}$. The beads were collected by pulse centrifugation and washed. The final pellet was resuspended in $30 \mu \mathrm{l} 1 \mathrm{x}$ sample buffer and heated to 95 ㅇ $\mathrm{C}$ for $5 \mathrm{~min}$. The beads were pelleted by centrifugation at $12,000 \mathrm{~g}$ for $20 \mathrm{sec}$ and the supernatant was used for Western blot analysis. 


\section{Immunoblotting}

Proteins were separated by SDS-PAGE and transferred to nitrocellulose membranes for Western Blotting. The membranes were probed with the relevant primary antibodies. The protein bands were visualized using enhanced chemiluminescence and immunoblot intensities were analyzed by densitometry. ${ }^{13}$

\section{Permeabilized myocyte preparations}

Ventricular myocytes were dissociated mechanically as previously described. ${ }^{20}$ The heart was perfused retrogradely $(1.6 \mathrm{ml} / \mathrm{min})$ with a $\mathrm{Ca}^{2+}$-free Hanks-HEPES buffered solution for $5 \mathrm{~min}$ at room temperature, then preskinned by perfusing with relaxing solution containing $1 \%$ Triton X-100 and protease inhibitors for 10 $\mathrm{min}$. Left ventricular strips (8-mm long, 2-mm width, $1 \mathrm{~mm}$ thick) were dissected from the same heart, frozen in liquid nitrogen for biochemistry or further skinned for 10 min at $4 \stackrel{\circ}{\circ}$ in relaxing solution containing $1 \%$ Triton X-100 for mechanical experiments. The strips were then blended in fresh ice-cold relaxing solution at $11,000 \mathrm{rpm}$ for $2-3 \mathrm{~s}$. The resulting suspension was further skinned in $0.3 \%$ Triton $\mathrm{X}-100$ solution for $6 \mathrm{~min}$ at room temperature.

Solutions: $\mathrm{Ca}^{2+}$ activating solutions were prepared by mixing relaxing ( $\mathrm{p} C \mathrm{Ca} 9.0$ ) and maximal activating ( $\mathrm{pCa} 4.5$ ) stock solutions. The relaxing and activating solutions contained $12 \mathrm{mM}$ phosphocreatine, $30 \mathrm{mM}$ imidazole, $1 \mathrm{mM}$ free $\mathrm{Mg}^{2+}, 10$ mM EGTA, $3.3 \mathrm{mM} \mathrm{Na}_{2} \mathrm{ATP}$, and $0.3 \mathrm{mM}$ dithiothreitol with pCa 9.0 (relaxing solution) and pCa 4.5 (maximal activating solution), pH 7.1. For PKD stimulation, cells were preincubated for $30 \mathrm{~min}$ at room temperature with $10 \mu \mathrm{g}$ of full length active PKD per $\mathrm{ml}$ of relaxing solution.

Force measurements: The procedure of cell attachment has been previously described. ${ }^{20}$ Skinned myocyte was attached to a piezoresistive strain gauge (AE801 sensor, Memscap, Crolle, France; $500 \mathrm{~Hz}$ unloaded resonant frequency, compliance of the strain gauge $0.03 \mu \mathrm{m} / \mu \mathrm{N}$ ) and to a stepper motor driven micromanipulator (MP-285, Sutter instrument company), cardiomyocyte at $2.0 \mu \mathrm{m}$ sarcomeric length (SL). Contractions were induced by application of solutions containing various pCa (from 6.2 to 4.5 ).

\section{Statistics}

Data are presented as mean \pm SEM. The paired Student's t-test and one-way ANOVA was used. When significant interactions were found, a Bonferroni post-hoc test was applied. A value of $\mathrm{P}<0.05$ indicates a statistical significance. 


\section{Results}

\section{Contraction increases PKD binding and phosphorylation on cMyBP-C}

It has been shown that in quiescent mouse myocardium, incubation with the catalytic subunit of PKD induces phosphorylation cMyBP-C-Ser302 (which is identical to Ser315 in rat). We previously demonstrated that contraction, induced by electric field stimulation (EFS), activates PKD in isolated rat cardiomyocytes. ${ }^{13}$ Also, the $F_{1} F_{0^{-}}$ ATPase inhibitor oligomycin, which induces a decrease in the cellular energy stores, similar to that observed during increased cardiac contraction, stimulates PKD activity. ${ }^{13}$ Since CMyBP-C has been shown to be one of the targets of PKD, ${ }^{4}$ we first investigated whether during contraction phosphorylation of $\mathrm{CMyBP}-\mathrm{C}$ is increased. Therefore, we measured phosphorylation of cMyBP-C-Ser315 in electric field stimulated rat cardiomyocytes. Indeed, EFS induces phosphorylation of cMyBP-C-Ser315 (Figure 1A). During EFS of rat cardiomyocytes, PKD interacts with cMyBP-C-Ser315 and it does this by directly interacting with cMyBP-C, as shown by immunoprecipitation (Figure 1B). In EFS treated cardiomyocytes, immunoprecipitation of PKD showed an increased binding to cMyBP-C-Ser315 (Fig. 1B). Reciprocally, in immunoprecipitation of cMyBP-C, PKD was co-immunoprecipitated with cMyBP-C, and the level of coimmunoprecipitated PKD was markedly higher in EFS treated cardiomyocytes than in non-stimulated (control) cardiomyocytes (Fig. 1B).

\section{Figure 1}

A

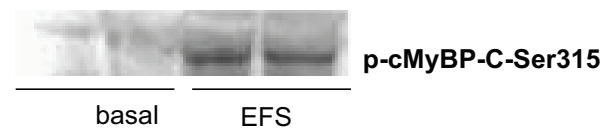

B

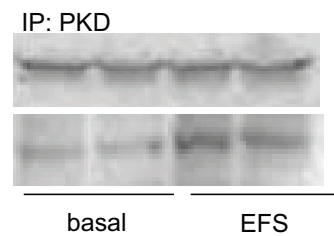

IB: PKD

IB: c-MyBP-C-ser315

IP: CMyBP-C

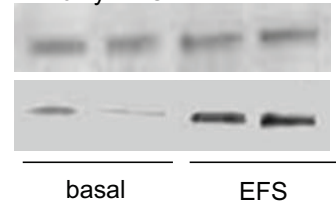

IB: C-MyBP-C

IB: PKD 
Fig. 1 Interaction between PKD and CMYBP-C upon increased contraction. Isolated cardiomyocytes were electrical field stimulated (EFS) at $4 \mathrm{~Hz}$ for $6 \mathrm{~min}$ and were compared to non-stimulated cardiomyocytes. (A) cMyBP-C-Ser315 becomes phosphorylated during EFS. (B) Upon cMyBP-C immunoprecipitation (IP), CMyBP-C and PKD were detected by immunoblotting (IB). Upon PKD immunoprecipitation (IP), cMyBP-CSer315 and PKD were detected by immunoblotting (IB). Results are representative of 3 independent experiments.

We also applied a pharmacological approach to inhibit activation of PKD in oligomycin treated and EFS treated cardiomyocytes, using staurosporin, a general PKC/ PKD inhibitor, calphostin-C, an inhibitor of all diacylglycerol-activated PKC- and PKD isoforms, and Gö6983, an inhibitor of conventional PKCs. Pretreatment of cardiomyocytes with staurosporin or calphostin-C, but not with Gö6983, resulted in a marked inhibition of CMyBP-C phosphorylation. Since only PKD, and not the main cardiac PKC isoforms, is activated upon oligomycin treatment in cardiomyocytes, ${ }^{13}$ these results suggest that CMyBP-C is a direct target of PKD action (Fig. 2) Together, these data demonstrate that in cardiomyocytes, PKD directly binds to cMyBP-CSer315, which is enhanced during contraction.

\section{Figure 2}

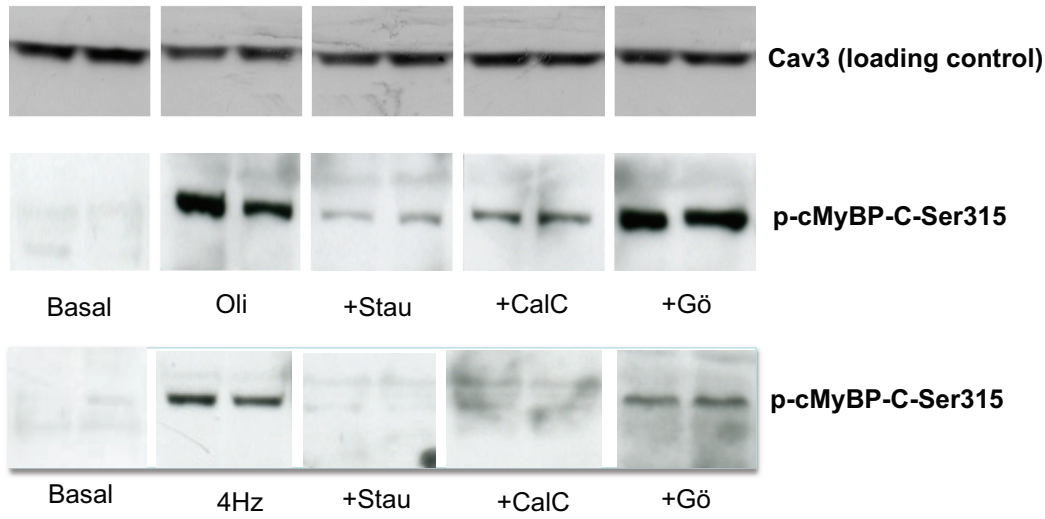

Fig. 2 Effects of PKC/PKD inhibitors on CMyBP-C phosphorylation in cardiomyocytes. Isolated rat cardiomyocytes were pre-incubated for $15 \mathrm{~min}$ at $37^{\circ} \mathrm{C}$ with or without addition of $1 \mu \mathrm{mol} / \mathrm{L}$ staurosporin (Stau), $2 \mu \mathrm{mol} / \mathrm{L}$ calphostin-C (CaIC) or $10 \mu \mathrm{mol} / \mathrm{L}$ Gö6983. Subsequently, cardiomyocytes were treated with $5 \mu \mathrm{mol} / \mathrm{L}$ oligomycin (Oli) or eletric field stimulated. Samples were immunoblotted against Ser315 phosphorylation of cMyBP-C and caveolin 3 (cav3) (loading control). Blot representative of 3 independent experiments. 


\section{cMyBP-C-Ser315 phosphorylation by PKD is workload dependent}

We further investigated the PKD-mediated regulation of cMyBP-C under increased workload conditions. We determined PKD-mediated cMyBP-C phosphorylation at different EFS frequencies in isolated rat cardiomyocytes. Compared with nonstimulated rat cardiomyocytes, EFS at $1 \mathrm{~Hz}, 2 \mathrm{~Hz}$ and $4 \mathrm{~Hz}$ increased PKD-Ser916 phosphorylation by 1.7-fold, 2.7-fold and 3.2-fold, and cMyBP-C-Ser315 phosphorylation by 3.1-fold, 4.4-fold and 5.1-fold, respectively (Fig. 3). The parallel upregulation in contraction-induced PKD and CMyBP-C phosphorylation strongly supports the concept that PKD-dependent phosphorylation is instrumental for the cardiomyocyte to adjust to an increase in workload.

\section{Figure 3}

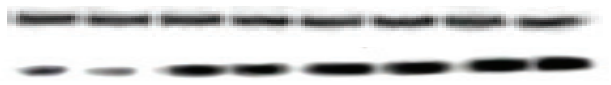

GAPDH

p-PKD-ser916
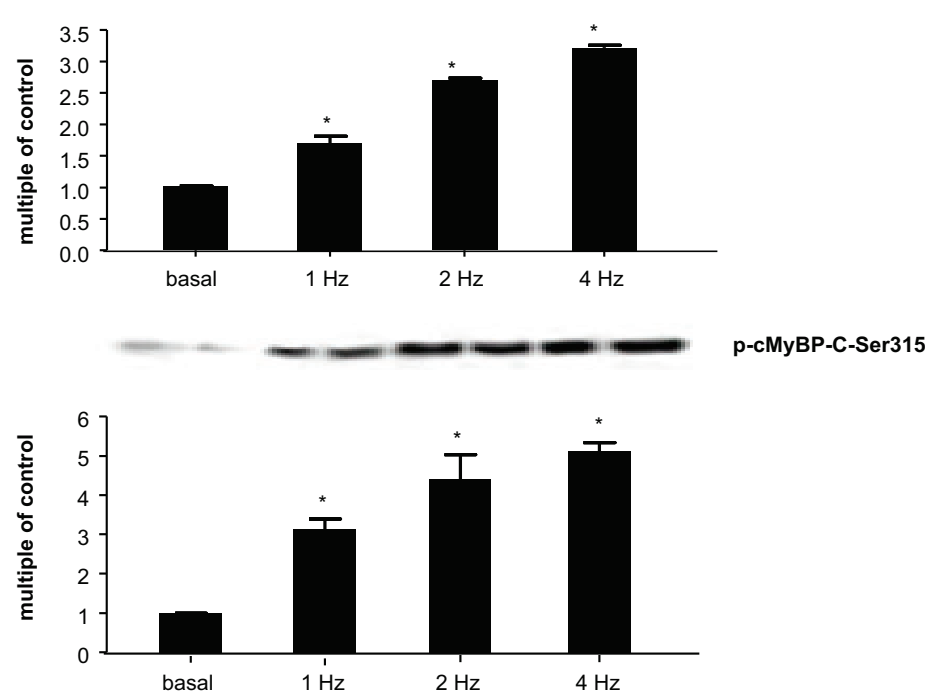

Fig. 3 PKD-Ser916 and CMYBP-C-Ser315 phosphorylation increases upon an increased contraction frequency. Isolated rat cardiomyocytes were electrically stimulated at $1 \mathrm{~Hz}, 2 \mathrm{~Hz}$ and $4 \mathrm{~Hz}$ for $6 \mathrm{~min}$. Samples were blotted against phospho-PKD-Ser916 and phospho-cMyBP-C-Ser315 and GAPDH (loading control). Values are expressed as multiple of control. Results are representative of 3 independent experiments. ${ }^{*} p<0.05$ vs non-treated cardiomyocytes, Student's-t-test. 

diomyocytes

The involvement of cMyBP-C in PKD-mediated effects on myofilament function was studied by using permeabilized ventricular cardiomyocytes from CMyBP-C-KO mice and from their wild type (WT) littermates. These cardiomyocytes were incubated with full-length active PKD. This PKD induced a 4-fold increase in cMyBP-C-Ser315 phosphorylation in permeabilized rat cardiomyocytes (Fig. 4A). To determine the physiological effect this cMyBP-C phosphorylation, the relation between $\mathrm{Ca}^{2+}$ activated tension and internal $\mathrm{Ca}^{2+}$ concentrations expressed as $\mathrm{pCa}\left(=-\log \left[\mathrm{Ca}^{2+}\right]\right)$ in permeabilized myocytes from WT and cMyBP-C KO mice was determined. PKD increased the absolute maximal $\mathrm{Ca}^{2+}$-activated tension of contraction $\left(\mathrm{T}_{\max }\right)$ and submaximal active tension starting from pCa 5.875 in WT, but not in CMyBP-C KO myocytes, showing that CMyBP-C is involved in the PKD-mediated increase in the tension of the muscle fiber during contraction. This raised the question whether this increase in force was due to an increase in myofilament $\mathrm{Ca}^{2+}$ sensitivity. PKD induced a leftward shift of the relative tension-pCa relationship curve and therefore increased in $\mathrm{PCa}_{50}$ in both WT and KO myocytes (Fig. 4B), indicating that the PKDmediated effect on $\mathrm{pCa}_{50}$ was independent of $\mathrm{cMyBP}-\mathrm{C}$ (Fig. 4B). Other contractile parameters such as Hill coefficient and passive tension were not affected by PKD treatment (data not shown).

To test whether the PKD-mediated effects on myofilament function were dependent on prior PKA activation, CMyBP-C KO and WT mice were pre-treated for 3 days with the non-selective $\beta$-blocker propranolol ( $0.5 \mathrm{~g} / \mathrm{l}$ in drinking water), which neutralizes PKA activity. In WT and KO mice pretreated with $\beta$-blocker, PKD did alter neither $\mathrm{pCa}_{50}$ nor Tmax (Fig. $4 \mathrm{C}$ ), indicating that the effect of PKD on $\mathrm{pCa}_{50}$ and $\mathrm{T}_{\max }$ is dependent on prior PKA activation. 


\section{Figure 4}

A

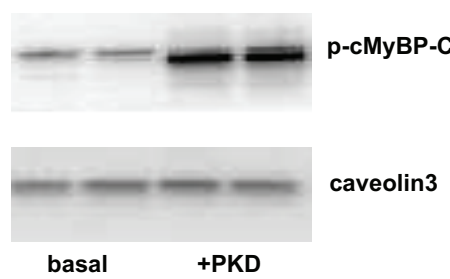

B
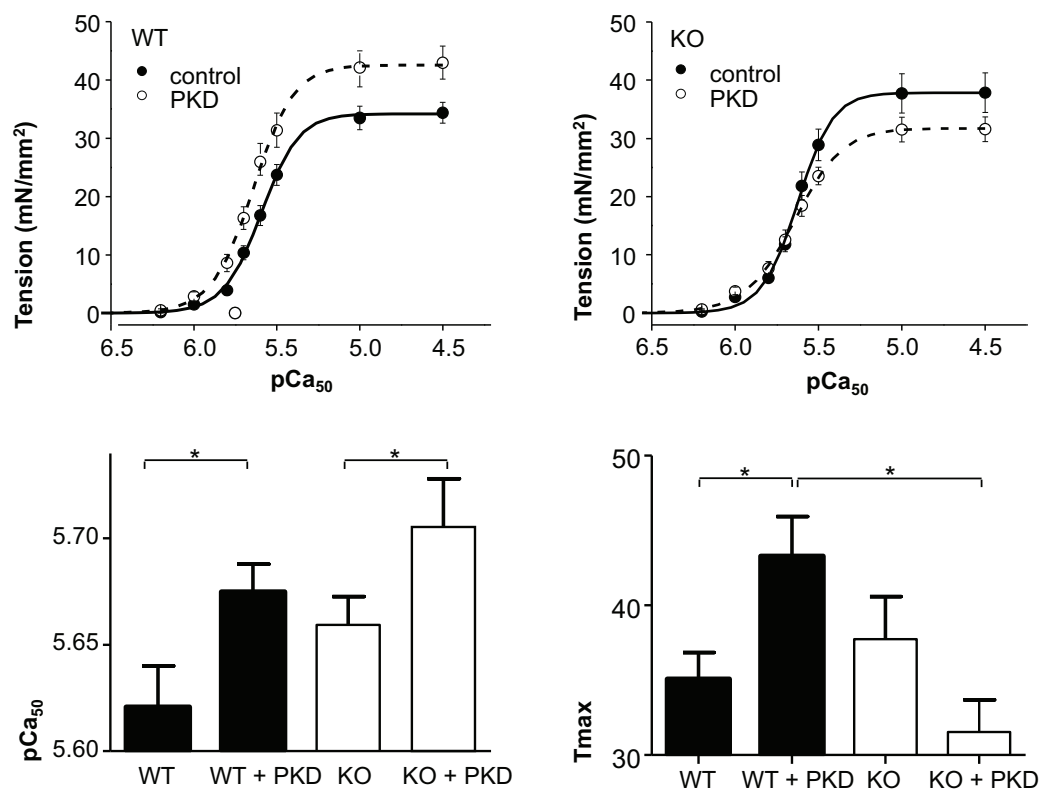

C
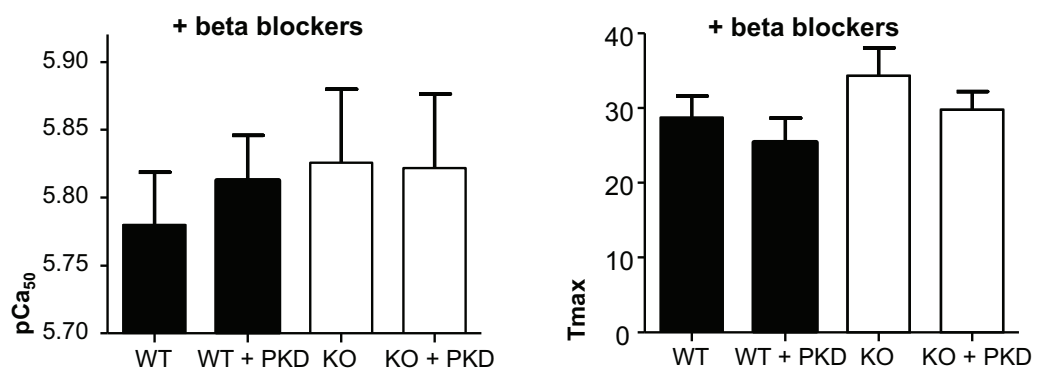

Fig. 4 PKD increases myofilament $\mathrm{Ca}^{2+}$-sensitivity and maximal tension of contraction

(A) Western blot analysis of p-MyBP-Ser315 in rat myofilaments incubated for $30 \mathrm{~min}$ with full-length active PKD (B) The relationship between $\mathrm{Ca}^{2+}$-activated tension and intracellular $\mathrm{Ca}^{2+}$ concentrations was measured in isolated, permeabilized cardiomyocytes at $2.0 \mu \mathrm{m}$ sarcomere length. The relationship was fitted with a modified Hill equation and the $\mathrm{pCa}$ at which half of the maximal tension is developed $\left(\mathrm{pCa}_{50}\right)$ 
was determined as an index of myofilament $\mathrm{Ca}^{2+}$ sensitivity as described previously. ${ }^{21}$ Incubation of WT cardiomyocytes with full length active PKD increased myofilament $\mathrm{Ca}^{2+}$ sensitivity, which is indicated by the shift toward the left of the curve and the increase of $\mathrm{pCa}_{50}$. Also, Incubation of WT cardiomyocytes with PKD increased maximal tension of contraction (Tmax), which is indicated by the upward shift of the curve. In cMyBP-C KO cardiomyocytes, incubation with full length active PKD still increased $\mathrm{pCa}_{50}$, while PKD was not able anymore to affect Tmax. (C) In both WT and CMyBP-C KO cardiomyocytes from mice pretreated with $\beta$-blockers, the PKD-mediated effects on $\mathrm{pCa}_{50}$ and Tmax were abolished. Results are expressed as mean \pm SEM, $n=12$ cells per group , ${ }^{*} p<0.05$.

\section{Discussion}

Phosphorylation of cMyBP-C is markedly decreased in experimental models of and in human heart failure, and as such it has been considered to be an important regulator of cardiac contractility. ${ }^{14,15,22}$ Understanding its regulation and the mechanisms by which cMyBP-C phosphorylation mediates cardiac contractile responses is critical in developing its potential as therapeutic target. We therefore investigated the PKD-mediated regulation of myofilament contraction through phosphorylation of $\mathrm{CMyBP}-\mathrm{C}$, and we show that $\mathrm{CMyBP}-\mathrm{C}$ is involved in PKD-mediated increase in myofilament activation, but not in the PKD-mediated $\mathrm{Ca}^{2+}$ sensitization of the myofilaments.

\section{During contraction, PKD phosphorylates CMyBP-C-Ser315 in rat cardiomy- ocytes}

This study is the first to show that during contraction cMyBP-C is a direct physiological substrate of PKD. Recently, it has been described that cMyBP-C- is a substrate of PKD in trabeculae under quiescent conditions. ${ }^{3,4}$ Our data show that, during contraction, PKD is activated, which is characterized by its Ser916 phosphorylation. This activation leads to an increased binding of PKD to cMyBP-C. Furthermore, in response to increased cardiac contractile activity (evoked by EFS of the cells), the Ser315 site of cMyBP-C becomes phosphorylated by PKD. During increased contraction frequencies, which simulated an increased workload, phosphorylation of both PKD-Ser916 and CMyBP-C-Ser315 increases similary, suggestion a functional relation.

\section{PKD-directed cMyBP-C phosphorylation alters the contractile kinetics}

Several studies have shown that exercise increases $\mathrm{Ca}^{2+}$ sensitivity of muscle tension and leads to a higher generated force. ${ }^{23-25}$ In exercising rats the maximal tension (Tmax) of the ventricular papillary muscle at optimal length became significantly higher. ${ }^{26}$ Also, it was found in porcine cardiac myofilaments that phosphorylation of CMyBP-C was increased in response to endurance exercise training. ${ }^{27}$ Here we report that phosphorylation of cMyBP-C-Ser315, by application of an activated fulllength PKD, results in an increase in submaximal activation and Tmax. The relevance of submaximal activation and Tmax increases with increasing workload. We showed 
that interaction of PKD with $\mathrm{CMyBP}-\mathrm{C}$ becomes stronger during increased contraction of cardiomyocytes. Furthermore, during a rise in contraction frequency (which simulates an increased workload), phosphorylation of both PKD and CMyBP-C was increased. Thus, the PKD-mediated effect on cMyBP-C becomes important in periods of increased contraction. The involvement of PKD in the force-frequency relationship is supported by data on cardiac-specific PKD KO mice that displayed normal contractility but were unable to respond to stress. ${ }^{28}$

Interestingly, cMyBP-C seems to be only important as a mediator in the PKD-driven increase in submaximal activation and Tmax. The PKD-mediated change in $\mathrm{Ca}^{2+}$ sensitivity was independent of cMyBP-C. The mechanism by which PKD activation leads to an increased $\mathrm{pCa}_{50}$ may imply phosphorylation of other contractile proteins. For instance, experiments in rabbit septal preparations revealed a significant frequency-dependent increase in myosin light chain 2 (MLC2) phosphorylation, which is known to increase myofilament $\mathrm{Ca}^{2+}$ sensitivity. ${ }^{29,}{ }^{30}$ Also, increased MLC2 phosphorylation and heart rate are associated with different levels of treadmill exercise or 8 -adrenergic stimulation and inhibition. ${ }^{31}$ This is in line with the concept that increased workload results in activation of PKD and subsequent $\mathrm{Ca}^{2+}$ sensitivity, independent of cMyBP-C. However, an earlier study showed that PKD reduced myofilament $\mathrm{Ca}^{2+}$ sensitivity in mice pretreated with propranolol, which prevents PKA activation. ${ }^{4}$ It is well known that PKA modulates cardiac contractility via intracellular $\mathrm{Ca}^{2+}$ movements. ${ }^{32}$ Upon activation, PKA is able to desensitize the muscle fiber for $\mathrm{Ca}^{2+}$-mediated force development and to increase cross-bridge cycle kinetics. ${ }^{4,33-35}$ In our study application of the $\beta$-adrenergic blocker propranolol led to a marked increase in $\mathrm{pCa}_{50}$, which could not be further increased by PKD. Also, in these cells, PKD was not able to increase myofilament Tmax. These results suggest that the effect of PKD on myofilaments is dependent on prior PKA phosphorylation. PKA phosphorylation of AKAP-Lcb may be necessary to release the activated PKD into the cytoplasm. ${ }^{2}$ The functional effect of PKD activation on the myofilaments without $\beta$-blocker treatment is of particular interest since it is known that, even more pronounced in rodents, there is a basal $\beta$-adrenergic tone which is increased during various conditions such as exercise ${ }^{36}$ and heart failure. ${ }^{37}$ This suggests that combined PKA and PKD activation become necessary during increased contraction or exercise to regulate the contraction mechanics. Given that, during increased contraction, PKD is also involved in enhanced glucose uptake ${ }^{13}$, it can be suggested that PKD activation helps the cardiomyocytes to cope with an increased workload by providing the cells with energy-rich substrate through an increase in glucose uptake. In this way PKD connects cardiac energetics and contractile mechanics (Fig. 5). 


\section{Figure 5}

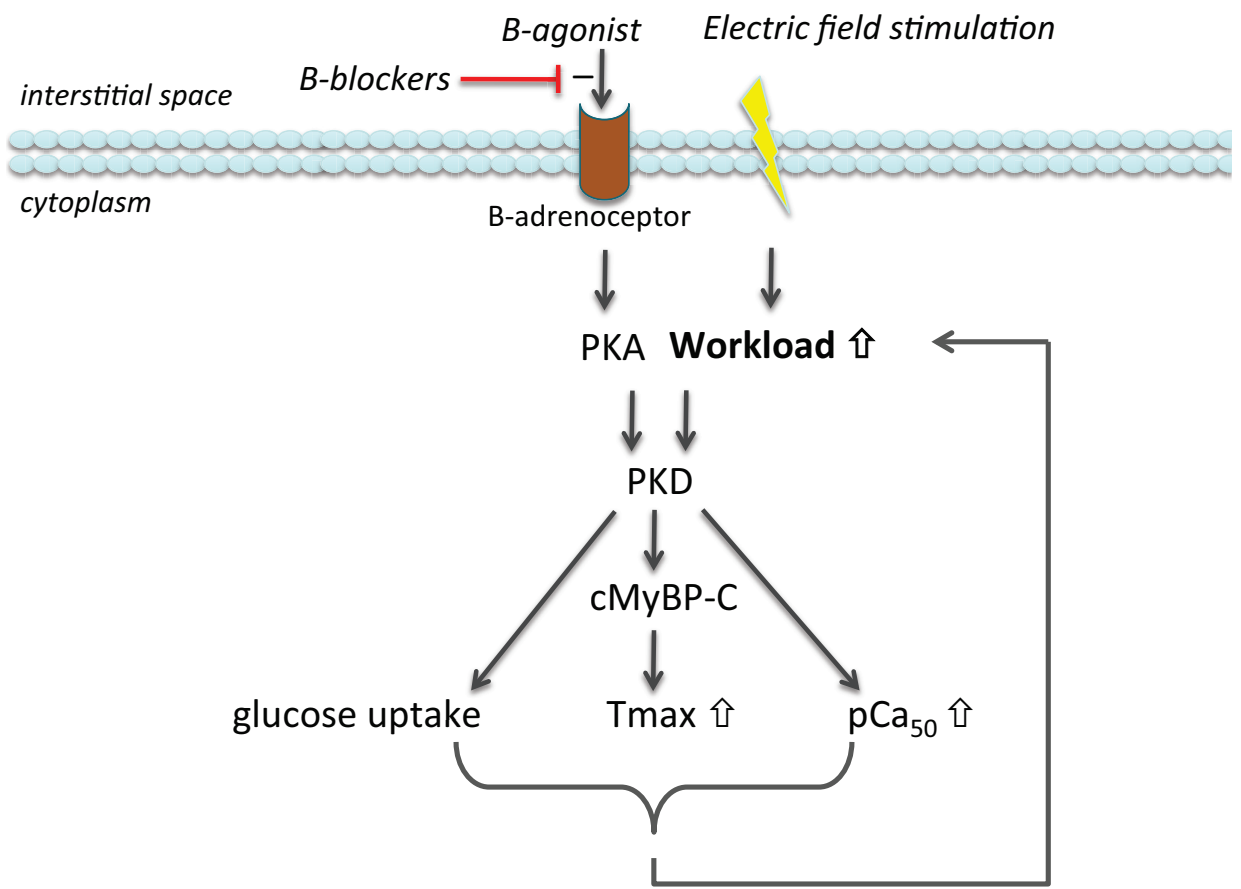

Fig. 5 Schematic representation of the consequences of an increased workload, which phosphorylates PKD and $C M y B P-C$. Upon a stress stimulus, like electric field stimulation, the workload of the cardiomyocytes will increase. This increase in workload will lead to phosphorylation of PKD and CMyBP-C. To enable coping with increased contractile demands, PKD facilitates contraction by increasing the $\mathrm{Ca}^{2+}$ sensitivity of contraction $\left(\mathrm{pCa}_{50}\right)$ and by inducing $\mathrm{CMyBP}-\mathrm{C}$ phosphorylation, which leads to an increase in maximal tension of contraction (Tmax). Furthermore, PKD provides the cardiomyocytes of additional energy-rich substrates by inducing glucose uptake. The latter increase is essential for the cardiomyocytes to handle the increased workload.

\section{Concluding remarks}

An increase in workload, induced by electric field stimulation, leads to the phosphorylation of both PKD and CMyBP-C in isolated cardiomyocytes. To facilitate coping with increased contractile demands, PKD increases the myofilament $\mathrm{Ca}^{2+}$ sensitivity and induces CMyBP-C phosphorylation, which leads to an increase in maximal tension of contraction. In addition, PKD increase glucose uptake for energy production. As a result, PKD is essential for the cardiomyocyte to properly respond to an increased workload and thus during periods of increased contractile activity when both the contractile and energy demands of the cardiomyocytes is highest. 


\section{Acknowledgements}

The authors would like to thank Thomas Eschenhagen (University Medical Center Hamburg-Eppendorf) for stimulating discussions, and Elisabeth Krämer, Birgit Geertz (University Medical Center Hamburg-Eppendorf) for there technical support. This study was funded by the transnational University Limburg (tUL) and the European Community (Integrated Project LSHM-CT-2004-005272, Exgenesis).

\section{Disclosures}

None 


\section{References}

1. Lint JV, Rykx A, Vantus T, et al. Getting to know protein kinase D. Int J Biochem Cell Biol. Jun 2002;34(6):577-581.

2. Carnegie GK, Smith FD, McConnachie G, et al. AKAP-Lbc nucleates a protein kinase D activation scaffold. Mol Cell. Sep 24 2004;15(6):889-899.

3. Cuello F, Bardswell SC, Haworth RS, et al. Protein kinase D selectively targets cardiac troponin $\mathrm{I}$ and regulates myofilament $\mathrm{Ca} 2+$ sensitivity in ventricular myocytes. Circ Res. Mar 30 2007;100(6):864-873.

4. Bardswell SC, Cuello F, Rowland AJ, et al. Distinct sarcomeric substrates are responsible for protein kinase D-mediated regulation of cardiac myofilament Ca2+ sensitivity and cross-bridge cycling. J Biol Chem. Feb 19 2010;285(8):5674-5682.

5. Lecarpentier $\mathrm{Y}$, Vignier N, Oliviero $\mathrm{P}$, et al. Cardiac Myosin-binding protein $\mathrm{C}$ modulates the tuning of the molecular motor in the heart. Biophys J. Jul 2008;95(2):720-728.

6. Pohlmann L, Kroger I, Vignier N, et al. Cardiac myosin-binding protein C is required for complete relaxation in intact myocytes. Circ Res. Oct 26 2007;101(9):928-938.

7. Carrier L, Schlossarek S, Willis MS, et al. The ubiquitin-proteasome system and nonsensemediated mRNA decay in hypertrophic cardiomyopathy. Cardiovasc Res. Jan 15;85(2):330338.

8. van Dijk SJ, Dooijes D, dos Remedios C, et al. Cardiac myosin-binding protein C mutations and hypertrophic cardiomyopathy: haploinsufficiency, deranged phosphorylation, and cardiomyocyte dysfunction. Circulation. Mar 24 2009;119(11):1473-1483.

9. Wu SC, Solaro RJ. Protein kinase C zeta. A novel regulator of both phosphorylation and dephosphorylation of cardiac sarcomeric proteins. J Biol Chem. Oct 19 2007;282(42):3069130698.

10. Yuan C, Guo Y, Ravi R, et al. Myosin binding protein C is differentially phosphorylated upon myocardial stunning in canine and rat hearts-- evidence for novel phosphorylation sites. Proteomics. Jul 2006;6(14):4176-4186.

11. de Tombe PP. Myosin binding protein C in the heart. Circ Res. May 26 2006;98(10):1234-1236.

12. Colson BA, Bekyarova $\mathrm{T}$, Locher MR, et al. Protein kinase A-mediated phosphorylation of CMyBP-C increases proximity of myosin heads to actin in resting myocardium. Circ Res. Aug 1 2008;103(3):244-251.

13. Luiken JJ, Vertommen D, Coort SL, et al. Identification of protein kinase D as a novel contraction-activated kinase linked to GLUT4-mediated glucose uptake, independent of AMPK. Cell Signal. Mar 2008;20(3):543-556.

14. El-Armouche A, Pohlmann L, Schlossarek S, et al. Decreased phosphorylation levels of cardiac myosin-binding protein- $\mathrm{C}$ in human and experimental heart failure. $\mathrm{J} \mathrm{Mol} \mathrm{Cell} \mathrm{Cardiol.} \mathrm{Aug}$ 2007;43(2):223-229.

15. Sadayappan S, Gulick J, Osinska H, et al. Cardiac myosin-binding protein-C phosphorylation and cardiac function. Circ Res. Nov 25 2005;97(11):1156-1163.

16. Carrier L, Knoll R, Vignier N, et al. Asymmetric septal hypertrophy in heterozygous cMyBP-C null mice. Cardiovasc Res. Aug 1 2004;63(2):293-304.

17. Sadayappan S, Osinska H, Klevitsky R, et al. Cardiac myosin binding protein $\mathrm{C}$ phosphorylation is cardioprotective. Proc Natl Acad Sci U S A. Nov 7 2006;103(45):16918-16923.

18. Van Lint JV, Sinnett-Smith J, Rozengurt E. Expression and characterization of PKD, a phorbol ester and diacylglycerol-stimulated serine protein kinase. J Biol Chem. Jan 20 1995;270(3):1455-1461.

19. Luiken JJ, van Nieuwenhoven FA, America G, et al. Uptake and metabolism of palmitate by isolated cardiac myocytes from adult rats: involvement of sarcolemmal proteins. $J$ Lipid Res. Apr 1997;38(4):745-758.

20. Cazorla O, Szilagyi S, Vignier N, et al. Length and protein kinase A modulations of myocytes in cardiac myosin binding protein C-deficient mice. Cardiovasc Res. Feb 1 2006;69(2):370-380.

21. Barefield D, Sadayappan S. Phosphorylation and function of cardiac myosin binding protein-C in health and disease. J Mol Cell Cardiol. May 2010;48(5):866-875.

22. Diffee GM, Chung E. Altered single cell force-velocity and power properties in exercise-trained rat myocardium. J Appl Physiol. May 2003;94(5):1941-1948. 
23. Natali AJ, Wilson LA, Peckham M, et al. Different regional effects of voluntary exercise on the mechanical and electrical properties of rat ventricular myocytes. J Physiol. Jun 15 2002;541(Pt 3):863-875.

24. Wisloff U, Loennechen JP, Currie S, et al. Aerobic exercise reduces cardiomyocyte hypertrophy and increases contractility, Ca2+ sensitivity and SERCA-2 in rat after myocardial infarction. Cardiovasc Res. Apr 2002;54(1):162-174.

25. Mole PA. Increased contractile potential of papillary muscles from exercise-trained rat hearts. Am J Physiol. Apr 1978;234(4):H421-425.

26. Hinken AC, Korte FS, McDonald KS. Porcine cardiac myocyte power output is increased after chronic exercise training. J Appl Physiol. Jul 2006;101(1):40-46.

27. Fielitz J, Kim MS, Shelton JM, et al. Requirement of protein kinase D1 for pathological cardiac remodeling. Proc Natl Acad Sci U S A. Feb 26 2008;105(8):3059-3063.

28. Silver PJ. Pharmacological modulation of cardiac and vascular contractile protein function. $J$ Cardiovasc Pharmacol. 1986;8 Suppl 9:S34-46.

29. Sweeney HL, Bowman BF, Stull JT. Myosin light chain phosphorylation in vertebrate striated muscle: regulation and function. Am J Physiol. May 1993;264(5 Pt 1):C1085-1095.

30. Fitzsimons DP, Bodell PW, Baldwin KM. Phosphorylation of rodent cardiac myosin light chain 2: effects of exercise. J Appl Physiol. Dec 1989;67(6):2447-2453.

31. Bers DM. Cardiac excitation-contraction coupling. Nature. Jan 10 2002;415(6868):198-205.

32. Hanft LM, McDonald KS. Sarcomere length dependence of power output is increased after PKA treatment in rat cardiac myocytes. Am J Physiol Heart Circ Physiol. May 2009;296(5):H1524-1531.

33. Layland J, Solaro RJ, Shah AM. Regulation of cardiac contractile function by troponin I phosphorylation. Cardiovasc Res. Apr 1 2005;66(1):12-21.

34. Matsuba D, Terui T, J OU, et al. Protein kinase A-dependent modulation of Ca2+ sensitivity in cardiac and fast skeletal muscles after reconstitution with cardiac troponin. J Gen Physiol. Jun 2009;133(6):571-581.

35. Paroo Z, Noble EG. Isoproterenol potentiates exercise-induction of $\mathrm{Hsp70}$ in cardiac and skeletal muscle. Cell Stress Chaperones. Sep 1999;4(3):199-204.

36. Slatton ML, Eichhorn EJ. Beta-blocker therapy for heart failure. Curr Opin Cardiol. May 1996;11(3):263-268.

37. Cazorla O, Lucas A, Poirier F, et al. The cAMP binding protein Epac regulates cardiac myofilament function. Proc Natl Acad Sci U S A. Aug 18 2009;106(33):14144-14149. 


\section{CHAPTER 6}

\section{Overexpression of AMPK-activated protein kinase or protein kinase D independently prevent lipid-induced insulin resistance in cardiomyocytes}

Ellen Dirkx ${ }^{1 *}$, Laura K.M. Steinbusch ${ }^{1^{*}}$, Nicole T.H. Hoebers ${ }^{1}$, Yeliz Angin ${ }^{1}$, Veronique Roelants $^{2}$, Benoit Viollet ${ }^{3}$, Michaela Diamant ${ }^{4}$, Guillaume van Eys ${ }^{1}$, D. Margriet Ouwens $^{5}$, Luc Bertrand ${ }^{2}$, Jan F.C. Glatz ${ }^{1}$, Joost J.F.P. Luiken ${ }^{1}$

${ }^{1}$ Department of Molecular Genetics, Cardiovascular Research Institute Maastricht (CARIM), Maastricht University, the Netherlands.

${ }^{2}$ Université catholique de Louvain, Institut de Recherche Expérimentale et Clinique,

Pole of Cardiovascular Research, Brussels, Belgium,

${ }^{3}$ Pasteur Institute, CNRS, UMR8104, Univ Paris Descartes, Paris, France.

${ }^{4}$ Diabetes Center, VU University Medical Center, Amsterdam, Netherlands.

${ }^{5}$ German Diabetes Centre, Düsseldorf, Germany.

*These authors contributed equally to this manuscript 


\begin{abstract}
In the healthy heart, not only insulin but also increased contractile activity stimulate glucose uptake. Upon increased contraction both AMP-activated protein kinase (AMPK) and protein kinase D (PKD) are activated and mediate the stimulation of glucose uptake into cardiomyocytes. High serum concentrations of lipids make the heart insulin resistant and will lead to diastolic dysfunction.

To test the potential of AMPK and PKD to compensate for the loss of insulinstimulated glucose uptake, both kinases were, by adenoviral transduction, overexpressed in primary cultures. To mimick insulin resistance and lipid loading, rat primary cardiomyocytes were cultured in the presence of high insulin (100 nM; HI) or high palmitate (palmitate/BSA: 3/1; HP), and glucose uptake, insulin sensitivity and lipid accumulation were measured. $\mathrm{HI}$ as well as HP reduced insulin sensitivity, and increased lipid storage. Overexpression of AMPK compensated for the reduced insulin sensitivity in both $\mathrm{HI}$ and HP-cultured cardiomyocytes, as measured by insulin-stimulated glucose uptake and Akt phosphorylation. However, increased AMPK levels could not reduce lipid accumulation in these culture conditions. In contrast, overexpression of PKD only compensated for the reduced insulin sensitivity in HP culture medium, but it did prevent lipid accumulation in both $\mathrm{HI}$ and HP culture conditions.

In conclusion, overexpression of both AMPK and PKD have the potential to prevent a reduction in insulin sensitivity in HI or HP cultured cardiomyocytes. However, only PKD reduced lipid accumulation under these culture conditions.
\end{abstract}




\section{Introduction}

The insulin resistant heart is characterized by impaired cardiac function and altered substrate preference towards increased long chain fatty acid (LCFA) utilization at the expense of glucose. ${ }^{1}$ In time, the greater influx of LCFA exceeds the mitochondrial $\beta$-oxidative capacity. ${ }^{2}$ This mismatch between LCFA uptake and oxidation results in the gradual intramyocellular build-up of triacylglycerols (TG) and various LCFA metabolites, leading to decreased insulin signaling and insulinstimulated glucose uptake. ${ }^{3}$ Over time, both lipid accumulation and insulin resistance contribute to altered energy metabolism and remodeling of the heart, which eventually, in the presence of cardiac stress such as ischemia, may lead to heart failure. ${ }^{4}$ Strategies that stimulate glucose uptake, or reduce LCFA uptake, independent of insulin signaling, may serve as strategies to normalize substrate uptake in the insulin resistant heart, and thereby protect against loss of contractile function. 5

In cardiomyocytes, glucose and LCFA uptake are mainly regulated by reversible glucose transporter 4 (GLUT4) and fatty acid transporter CD36 translocation between intracellular storage compartments and the sarcolemma. ${ }^{1,7}$ Insulin, as well as increased contractile activity, independently stimulate glucose uptake into cardiomyocytes. $^{8}$ Since insulin signaling is blunted during insulin resistance, recent research has focused on activation of contraction-induced signaling for stimulation of glucose uptake. ${ }^{9}$

It is well documented that during contraction-induced signaling AMP-activated protein kinase (AMPK) is activated. ${ }^{9}$ Activation of AMPK occurs through phosphorylation by upstream kinases, such as LKB1, in combination with binding of AMP to one of the regulatory subunits. ${ }^{9}$ In mice overexpressing a dominantnegative mutant of AMPK $\alpha$, it has been demonstrated that AMPK is necessary for contraction-induced glucose and LCFA uptake. ${ }^{10}$ Several AMPK-activating agents, such as the biguanide metformin and resveratrol, currently serve as blood-glucose lowering agents. ${ }^{11}$ Although metformin improved glycemic control, it did not improve cardiac function in type 2 diabetes patients. ${ }^{12}$ Activation of AMPK by resveratrol was shown to improve health and survival when given to mice on a highcaloric diet. $^{13,14}$

Recently, we showed that also protein kinase D (PKD) is contraction activated and stimulates glucose uptake in rat cardiomyocytes ${ }^{15}$. PKD catalytic activity becomes apparent by its autophosphorylation at Ser916 ${ }^{16}$. Pharmacological inhibition of PKD activity did not affect AMPK-Thr172-phosphorylation but reduced glucose uptake to basal levels ${ }^{15}$. In addition, it was shown that contraction-activated PKD does not affect LCFA uptake into cardiomyocytes (Chapter 3). This makes PKD a new player in the regulation of cardiac metabolism. 
Because both AMPK and PKD mediate contraction-stimulated glucose uptake, both kinases may restore glucose uptake in insulin-resistant cells. Whether AMPK or PKD can prevent cardiac lipid loading and insulin resistance, remains elucive. In this study we showed that in cardiomyocytes, cultured under high lipid or high insulin conditions, adenoviral overexpression of AMPK or PKD might restore insulin sensitivity.

\section{Materials and Methods}

\section{Materials}

Chemicals: $\left[{ }^{3} \mathrm{H}\right]$ deoxyglucose was obtained from GE Healthcare (Little Chalfont, UK). Laminin and insulin were purchased from Sigma (Saint Louis, USA). Bovine serum albumin (BSA) (fraction V), dependent on the application, was derived from MP Biomedicals (Irvine, USA) (for cell isolation and incubation purposes), or from Sigma (other purposes). Collagenase type II was from Worthington (Freehold, USA).

Animals: Male Lewis rats, 200-250 gram, were purchased from Charles River laboratories and used for culturing isolated cardiomyocytes. All animal procedures were approved by the Experimental Animal Committee of Maastricht University, NL.

\section{Adenovirus amplification}

Control adenovirus containing enhanced green fluorescent protein (GFP) (control), a recombinant adenovirus encoding a truncated form of AMPK $\alpha 2$ (AMPK $\alpha 2$ 1-312) and GFP (AdAMPK), ${ }^{19,20}$ and a recombinant adenovirus encoding full-length wildtype mouse PKD1 and EGFP (AdPKD) ${ }^{21}$ were used. Adenoviruses were amplified amplified in HEK293 cells and purified over $\mathrm{CsCl}_{2}$ gradients as previously described by Luo et al. ${ }^{22}$ Optimal multiplicity of infection (MOI) was determined by fluorescence microscopy and western blotting for downstream targets of both AMPK and PKD (Suppl. Fig. 1-2). MOI 40 was chosen for AdAMPK and MOI 10 was chosen for AdPKD.

\section{Isolating and Culturing of rat cardiomyocytes}

Rat cardiomyocytes were isolated using a Langendorff perfusion system, as previously described ${ }^{17,18}$ with the only difference being the sterile conditions that were taken into account for rat cardiomyocyte isolations.

After isolation of cardiomyocytes, 200,000 cells were seeded per well in laminin coated 6-well plates and after 90 minutes adhesion medium (control medium without $\left.\mathrm{Ca}^{2+}\right)$ was replaced with control medium $(20 \mu \mathrm{M}$ palmitate, palmitate:BSA $0.3: 1)$, high insulin medium (100 $\mathrm{nM}$ ) or high palmitate medium (200 $\mu \mathrm{M}$, palmitate:BSA 3:1). Cells were cultured according to the method described by Volz 
et al. $^{23}$ in modified serum-free medium M199 containing carnitine, creatine and taurine. $^{23}$ Culturing adult cardiac myocytes does not affect their insulin responsiveness. ${ }^{24}$ During the experiment the cells were maintained in an incubator $\left(37^{\circ} \mathrm{C}, 5 \% \mathrm{CO}_{2}\right)$ and were used for experiments $48 \mathrm{~h}$ after seeding and infection. Primary rat cardiomyocytes were infected with control, AdAMPK, or AdPKD 90 minutes after seeding the cardiomyocytes. Cardiomyocytes were cultured for $48 \mathrm{~h}$ because of optimal combination of $\mathrm{MOI}$ and transfection time (Suppl. Fig. 1-2).

\section{Measurement of glucose uptake}

Uptake of $\left[{ }^{3} \mathrm{H}\right]$ deoxyglucose into cardiac myocytes was measured as previously described for cardiomyocytes in culture. ${ }^{25}$ In short, after 2 days of culturing, cardiomyocytes were washed and were allowed to recover for 30 minutes from short-term insulin effects. This recovery time is sufficiently long to dampen all shortterm insulin effects (see ${ }^{26}$ and Angin and Steinbusch et al. unpublished). Afterwards, one portion of the cells was exposed to insulin (100 nM) for 15 minutes to be able to compare basal uptake with insulin-stimulated uptake. Then, radioactively-labeled glucose was added for 10 minutes, cells were washed, lysed and radioactivity was measured with a $\beta$-counter.

\section{Triacylglycerol stores}

Intramyocellular lipids were determined after 2 days of culturing in lysed cells as described previously. ${ }^{27}$ Triacylglycerol bands were detected with a Molecular Imager (ChemiDoc XRS, BioRad) and analyzed with Quantity One ${ }^{\circledR}$ (BioRad).

\section{Protein detection}

Rat cardiomyocytes were assayed after 2 days of culturing and a recovery of 30 minutes from acute insulin effects. Afterwards, cells were exposed to insulin (100 $\mathrm{nM})$ for 15 minutes to be able to compare basal-phosphorylation to insulinstimulated phosphorylation. Cells were lysed in sample buffer and used for protein detection by Western blotting, as previously described ${ }^{28}$. Primary antibodies against phospho-Ser473-Akt, Akt, phospho-Thr172-AMPK and phospho-Ser22/23Tnl from Cell Signaling (Danvers, MA), CD36 from GenTex Inc. (San Antonio, TX, USA), GLUT4 from Abcam (Cambridge, USA), phospho-Ser79-ACC from Upstate and caveolin3 from BD Transduction laboratories, were used

\section{Statistics}

All data are presented as means \pm S.E.M. Statistical difference between groups was evaluated by One-Way ANOVA, 2-Way ANOVA or Student t-test by using GraphPad Prism. P values equal to or less than 0.05 were considered significant. 


\section{Results and Discussion}

To test the effect of AMPK or PKD overexpression in cardiac insulin resistant, rat primary cardiomyocytes were exposed to a high concentration of insulin $(\mathrm{HI}$ medium) or to a high concentration of palmitate (HP medium) to induce lipotoxicity and insulin resistance by two different conditions.

\section{Effect of AMPK or PKD overexpression on glucose uptake, insulin sensitivity and lipid storage in control cells}

Cardiomyocytes were cultured for $48 \mathrm{~h}$ to achieve optimal combination of $\mathrm{MOI}$ and transfection time. Western blotting showed that overexpression of AMPK (using AdAMPK) did not affect PKD phosphorylation and overexpressing PKD (using AdPKD) did not affect AMPK phosphorylation (Fig. 1A). Overexpression of AMPK increased phosphorylation of its downstream substrate acetyl CoA carboxylase (ACC), but did not affect phosphorylation of a downstream target of PKD, troponin I (Tnl). Overexpression of PKD increased Tnl phosphorylation, but did not affect ACC phosphorylation (Fig. 1B). This indicates that both kinases were successfully overexpressed in primary cardiomyocyte cultures. Also, these data are in line with results decribed in chapter 3, demonstrating that PKD and AMPK do not function upstream or downstream from each other.

\section{Figure 1}

A

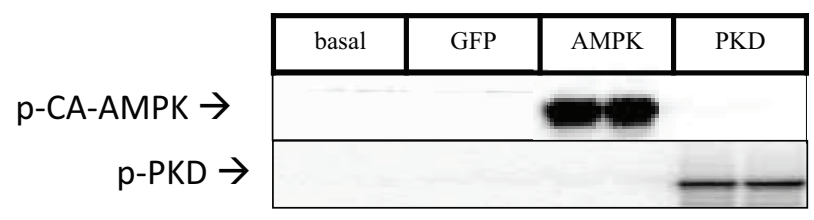

B

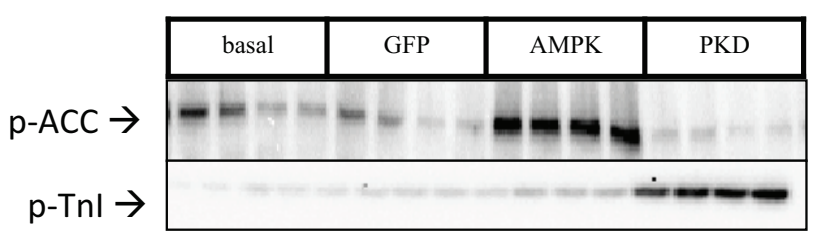

Fig. 1 Western blot images of cultured primary rat cardiomyocytes transfected with adenovirus containing green fluorescent protein (GFP), constitutively active AMP-activated protein kinase (AMPK) and protein kinase $D(P K D)$. (A) The truncated form of AMPK is present at $35 \mathrm{kDa}$, comprising the catalytical domain was detected in AdAMPK treated cells. In cells treated with AdPKD, PKD generated from the adenoviral construct was phosphorylated, as shown by a phospho-PKD-Ser916 antibody. (B) The downstream target 
of AMPK acyl-CoA carboxylase (ACC) was conciderably stronger phosphorylated in cells transfected with AMPK. The downstream target of PKD troponinl (Tnl) was only phosphorylated in cells transfected with PKD.

Cardiomyocytes cultured for $48 \mathrm{~h}$ in control medium, and transduced with GFP adenovirus, displayed insulin-stimulated glucose (1.8-fold) (Figures 2A), to a similar extent as seen in previous short-term substrate uptake studies with suspensions of freshly isolated cardiomyocytes. ${ }^{29}$ These results show that during culturing, the insulin sensitivity was maintained.

In line with previous work, ${ }^{10,15}$ overexpression of either AMPK or PKD increased basal glucose uptake (Fig. 2A) (2.3-fold and 3.3-fold, respectively). The insulinstimulated glucose uptake was retained upon overexpression of either kinase (Fig. 2A). To examine insulin sensitivity at the level of the insulin-signaling cascade we measured insulin-stimulated Akt. AdAMPK significantly increased basal and insulinstimulated Akt phosphorylation in cultured cardiomyocytes (3.9 and 3.0-fold, respectively), which is in line with previous results from experiments with cardiomyocyte suspensions. ${ }^{30}$ In contrast, AdPKD did not affect phosphorylation of Akt (Figure 2B). Similar AMPK-mediated effects on Akt phosphorylation have been obtained previously by using AMPK activators like metformin, phenformin or oligomycin. $^{31,32}$ Transfecting cardiomyocytes with adAMPK or adPKD did not significantly alter intramyocardial TG content (Fig. 2C) in normal insulin sensitive cardiomyocytes. However, there seems to be a trend of adPKD to increase intramyocardial TG content. In mice which overexpress PKD in the heart (caPKD mice) this effect of PKD overexpression on the intramyocardial TG content is significant (Chapter 7). Together, these results show that both AdAMPK and AdPKD are able to enhance glucose uptake into cultured cardiomyocytes. 


\section{Figure 2}

A

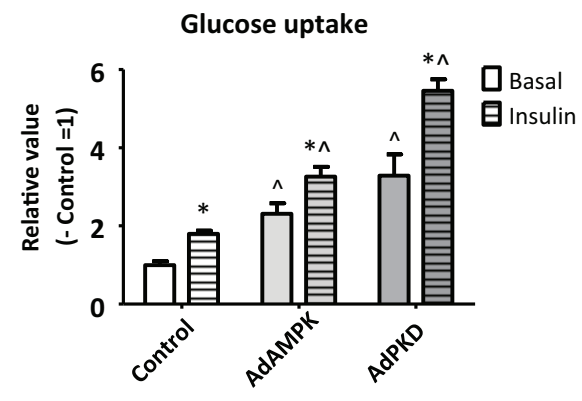

B

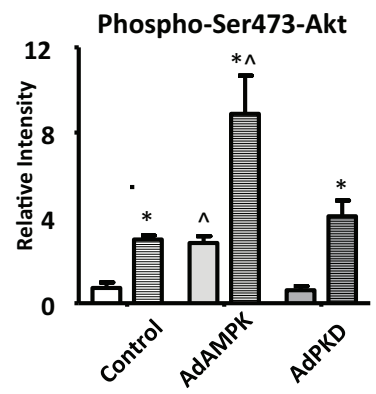

C

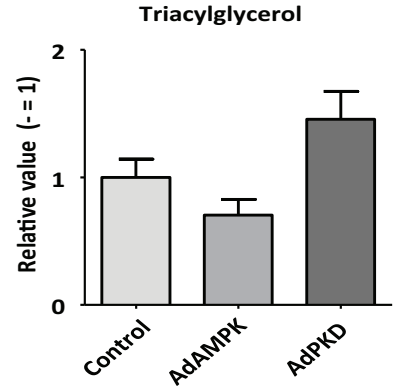

Fig. 2 Effect of overexpression of adenoviral constitutively active AMP-activated protein kinase (AdAMPK) or protein kinase $D(A d P K D)$ on metabolic parameters in cultured primary rat cardiomyocytes. (A) Glucose uptake measured by uptake of radioactive labeled substrate, basal (open bars) and insulin-stimulated (striped bars). GFP= adenovirus containing green fluorescent protein (B) Akt phosphorylation measured by western blotting, (C) triacylglycerol stores measured by HPTLC. Statistical analysis was done by OneWay with Bonferroni post-tests and Student t-test, ${ }^{*}$ Insulin effect $p<0.05, \wedge$ adenovirus effect $p<0.05$. 
For inducing insulin resistance, cardiomyocytes were exposed to a high insulin (HI) or a high palmitate (HP)-containing medium. HI or HP cultured cardiomyocytes showed no change in basal glucose uptake (Fig. 3). In agreement with previous work, ${ }^{31,32} \mathrm{HI}$-medium blocked insulin-stimulated glucose uptake entirely. Insulinstimulated glucose uptake was also abrogated in cardiomyocytes cultured in HPmedium (Fig. 3).

\section{Figure 3}

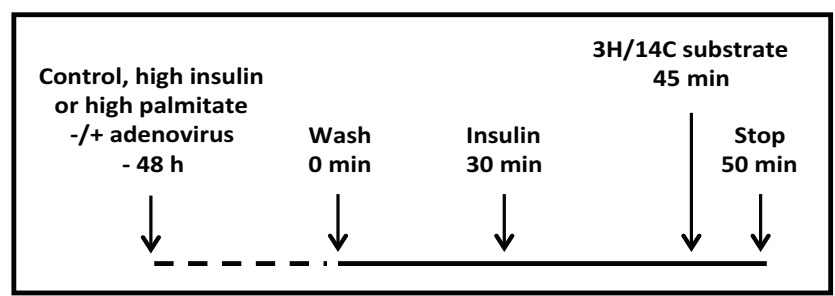

Glucose uptake

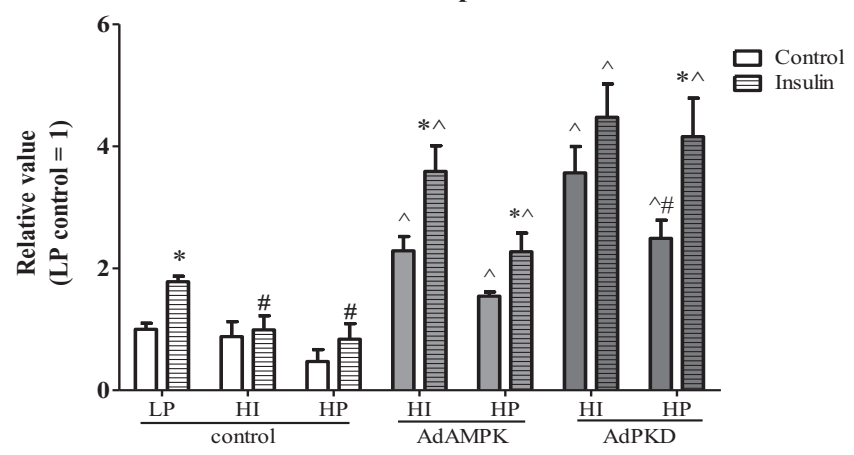

Fig. 3 Glucose uptake into rat primary cardiomyocytes cultured in control/low palmitate (LP), high insulin (HI) or high palmitate (HP) medium without or with the presence of green fluorescent protein adenovirus (GFP), constitutively active AMP-activated protein kinase adenovirus (AdAMPK) or protein kinase D adenovirus (AdPKD). Statistical analysis was done by One-Way ANOVA with Bonferroni post-tests and student t-test, $*$ Insulin effect $p<0.05, \wedge$ effect of adenoviral constructs, $p<0.05$, \# medium effect $p<0.05$.

Also, insulin-stimulated Akt phosphorylation was completely lost in HI-mediumcultured cardiomyocytes, and markedly reduced (by $42 \%, \mathrm{P}<0.05$ ) in $\mathrm{HP}$-mediumcultured cardiomyocytes cultured in high palmitate medium (Fig. 4). Both HI- and HP-media increased TG stores 1.9-fold and 2.9-fold, respectively (Fig. 5). 


\section{Figure 4}

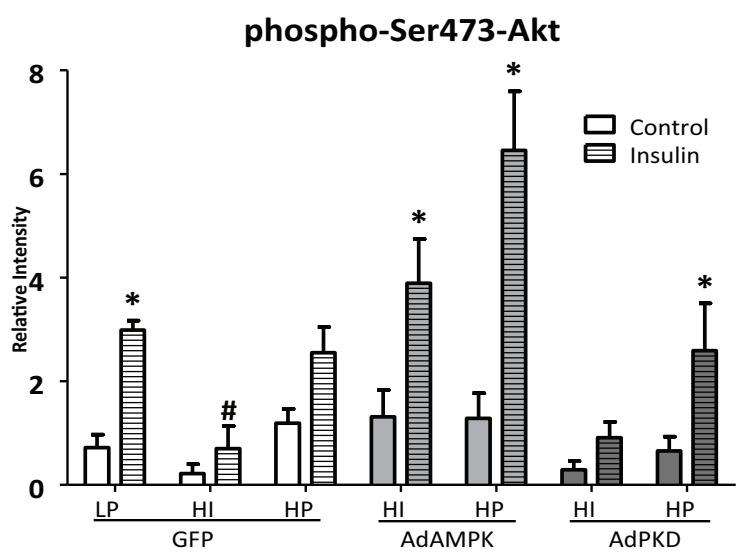

Fig. 4 Quantification of western blot images for basal and insulin-stimulated Akt-Ser473 phosphorylation in cultured primary rat cardiomyocytes transfected with green fluorescent protein adenovirus (GFP), constitutively active AMP-activated protein kinase adenovirus (AdAMPK) or protein kinase $D$ adenovirus $(A d P K D)$. Statistical analysis was done by One-Way ANOVA with Bonferroni post-tests and Student t-test, $*$ Insulin effect $p<0.05, \wedge$ adenovirus effect $p<0.05$.

Thus, both HI- and HP-media induce lipid accumulation and reduce insulin sensitivity in cardiomyocytes. The increased lipid accumulation in both $\mathrm{HI}$ - and HP-media might be a compensation for the reduction of glucose uptake (Fig. 3). On the other hand, an underlying mechanism for the increased lipid acculumation observed in HP culture conditions might be due to a permanent relocation of CD36 to the sarcolemma (Angin, Steinbusch et al., unpublished data), which leads to increased LCFA uptake and lipid storage.

\section{Figure 5}

\section{Triacylglycerol}

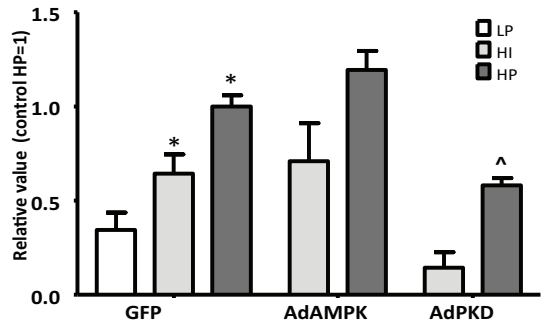

Fig. 5 Triacylglycerol stores measured by HPTLC in cell lysates from primary rat cardiomyocytes cultured in control/low palmitate (LP), high insulin (HI) or high palmitate (HP) medium in the presence green fluorescent protein adenovirus (GFP), adenovirus containing constitutively active AMP-activated protein kinase (AMPK) or protein kinase $D(P K D)$. Statistical analysis was done by 2-Way ANOVA with Bonferroni post-tests, $\mathrm{p}<0.05, *$ vs. LP in same group. ${ }^{\wedge}$ effect of adenovirus construct $p<0.05$. 


\section{Does AMPK overexpression prevent lipid accumulation and insulin resistance in cardiomyocytes cultured under insulin resistance-inducing conditions?}

AdAMPK enhanced basal glucose uptake in both $\mathrm{HI}$ and HP-medium-cultured cardiomyocytes. Also, in these culture conditions, insulin is still able to significantly induce glucose uptake (Fig. 3). In addition, culturing cardiomyocytes with AdAMPK maintained insulin-stimulated Akt-phosphorylation in both $\mathrm{HI}$ and HP media. Previously, it was shown that AMPK activation (by biguanides or oligomycin) or AMPK adenoviral-overexpression restores insulin-stimulated Akt-Ser473/Thr308phosphorylation and glucose uptake in cardiomyocytes cultured in HI-medium. ${ }^{31}$ Here we show that AdAMPK treatment of cardiomyocytes cultured in HP-medium also maintains insulin sensitivity by increasing insulin stimulated Akt phosphorylation and glucose uptake (Fig. 4). It has been shown that mTOR inhibition of insulin-signalling is increased in insulin resistant cells, and that inhibiting mTOR signalling (via AMPK) is sufficient to increase insulin sensitivity in term of Akt phosphorylation. ${ }^{36}$

Finally, AdAMPK treatment did not prevent TG accumulation in cardiomyocytes cultured in HP/HI-media. AdAMPK treatment might additionally activate LCFA oxidation, which prevents the LCFA from being stored. As mentioned in the introduction, the greater influx of LCFA exceeds the mitochondrial $\beta$-oxidative capacity during lipid oversupply, ${ }^{37}$ and hence AdAMPK treatment is not able to further activate the mitochondria to oxidize more LCFA.

From these results we conclude that AdAMPK treatment preserves insulin sensitivity in cardiomyocytes cultured in HI or HP culture conditions, but does not prevent lipid accumulation.

\section{Effect of PKD overexpression on substrate uptake, insulin sensitivity and lipid storage}

AdPKD treatment of $\mathrm{HI}$ or HP cultured cardiomyocytes increased basal glucose uptake and maintains insulin-stimulated glucose uptake in HP cultured cardiomyocytes, but not $\mathrm{HI}$ cultured cardiomyocytes (Fig. 3). Also, AdPKD treatment did not alter Akt phosphorylation under both conditions (Fig. 4). These results suggest that the preventive action of AdPKD on insulin-stimulated glucose uptake could not be explained by altered Akt-phosphorylation. AdPKD markedly reduced the storage of TG in cardiomyocytes cultured in both insulin resistance-inducing media (Fig. 5). Since the role of PKD in cellular metabolism is a relatively novel research subject, downstream PKD targets have been incompletely mapped. 
However, in caPKD mice it was shown that PKD overexpression induces intracellular TG accumulation and that this effect of PKD was inhibited by feeding the caPKD mice a high fat diet (chapter 7). Also, a high fat diet decreases PKD protein concentration (Chapter 8). This way PKD creates a feedback-loop in lipid storage. Together, this could explain the decrease of TG pools in HP cultured and adPKD treated cardiomyocytes.

Thus, it can be concluded that in cardiomyocytes treated with AdPKD and cultured under HP-media conditions, the lipid accumulation was neutralized and insulininduced glucose uptake was preserved.

\section{Limitations of this study}

Adenoviral transduction with AMPK and PKD cDNAs results in a massive expression of these kinases (Fig.1). Normally such high levels of expression are in time counteracted by compensatory systems. However, these are acute experiments and therefore time for such a response lacks. Thus, these experiments provide insight on the direct respons to a strong rise in AMPK or PKD, but the information is of limited value for processes such as insulin resistance since this develops over prolonged period of time.

\section{Conclusion}

AMPK and PKD each have potential to prevent insulin sensitivity in cultured cardiomyocytes and they probably do so through different mechanisms. This suggests that both the AMPK and PKD pathway could be suitable targets for prevention insulin resistance.

\section{Acknowledgments}

This work was supported by the Dutch Diabetes Research Foundation (Grant: 2006.00.044), and the EU European Cooperation in the field of Scientific and Technical Research (COST) Action BM0602 (Adipose tissue: A key target for prevention of the metabolic syndrome), by grants from the Fonds National de la Recherche Scientifique et Médicale, Belgium. LB is Research Associate of the Fonds National de la Recherche Scientifique, Belgium, by the Association Française contre les Myopathies (AFM) and Association pour l'Etude des Diabètes et des Maladies Métaboliques (ALFEDIAM).

\section{Disclosures}

None 


\section{References}

1. Glatz JFC, Luiken JJFP, Bonen A. Membrane fatty acid transporters as regulators of lipid metabolism: implications for metabolic disease. Physiol Rev. Jan 2010;90(1):367-417.

2. Bonen A, Holloway GP, Tandon NN, et al. Cardiac and skeletal muscle fatty acid transport and transporters and triacylglycerol and fatty acid oxidation in lean and Zucker diabetic fatty rats. Am J Physiol Regul Integr Comp Physiol. Oct 2009;297(4):R1202-1212.

3. Borradaile NM, Schaffer JE. Lipotoxicity in the heart. Curr Hypertens Rep. Dec 2005;7(6):412417.

4. Taegtmeyer $\mathrm{H}$, McNulty $\mathrm{P}$, Young ME. Adaptation and maladaptation of the heart in diabetes: Part I: general concepts. Circulation. Apr 9 2002;105(14):1727-1733.

5. Steinbusch LK, Luiken JJ, Vlasblom R, et al. Absence of fatty acid transporter CD36 protects against Western-type diet-related cardiac dysfunction following pressure overload in mice. Am J Physiol Endocrinol Metab. Jun 282011.

6. Glatz JF, Bonen A, Ouwens DM, et al. Regulation of sarcolemmal transport of substrates in the healthy and diseased heart. Cardiovasc Drugs Ther. Dec 2006;20(6):471-476.

7. Zorzano A, Sevilla L, Camps M, et al. Regulation of glucose transport, and glucose transporters expression and trafficking in the heart: studies in cardiac myocytes. Am J Cardiol. Aug 4 1997;80(3A):65A-76A.

8. Lopaschuk GD, Ussher JR, Folmes CD, et al. Myocardial fatty acid metabolism in health and disease. Physiol Rev. Jan 2010;90(1):207-258.

9. Steinberg GR, Kemp BE. AMPK in Health and Disease. Physiol Rev. Jul 2009;89(3):1025-1078.

10. Habets DD, Coumans WA, El Hasnaoui M, et al. Crucial role for LKB1 to AMPKalpha2 axis in the regulation of CD36-mediated long-chain fatty acid uptake into cardiomyocytes. Biochim Biophys Acta. Mar 2009;1791(3):212-219.

11. Hawley SA, Ross FA, Chevtzoff C, et al. Use of cells expressing gamma subunit variants to identify diverse mechanisms of AMPK activation. Cell Metab. Jun 9 2010;11(6):554-565.

12. van der Meer RW, Rijzewijk LJ, de Jong HW, et al. Pioglitazone improves cardiac function and alters myocardial substrate metabolism without affecting cardiac triglyceride accumulation and high-energy phosphate metabolism in patients with well-controlled type 2 diabetes mellitus. Circulation. Apr 21 2009;119(15):2069-2077.

13. Baur JA, Pearson KJ, Price NL, et al. Resveratrol improves health and survival of mice on a high-calorie diet. Nature. Nov 16 2006;444(7117):337-342.

14. Um JH, Park SJ, Kang H, et al. AMP-activated protein kinase-deficient mice are resistant to the metabolic effects of resveratrol. Diabetes. Mar 2010;59(3):554-563.

15. Luiken JJ, Vertommen D, Coort SL, et al. Identification of protein kinase D as a novel contraction-activated kinase linked to GLUT4-mediated glucose uptake, independent of AMPK. Cell Signal. Mar 2008;20(3):543-556.

16. Rykx A, De Kimpe L, Mikhalap S, et al. Protein kinase D: a family affair. FEBS Lett. Jul 3 2003;546(1):81-86.

17. Luiken JJ, van Nieuwenhoven FA, America G, et al. Uptake and metabolism of palmitate by isolated cardiac myocytes from adult rats: involvement of sarcolemmal proteins. J Lipid Res. Apr 1997;38(4):745-758.

18. Habets DD, Coumans WA, Voshol PJ, et al. AMPK-mediated increase in myocardial long-chain fatty acid uptake critically depends on sarcolemmal CD36. Biochem Biophys Res Commun. Mar 30 2007;355(1):204-210.

19. Foretz M, Ancellin N, Andreelli F, et al. Short-term overexpression of a constitutively active form of AMP-activated protein kinase in the liver leads to mild hypoglycemia and fatty liver. Diabetes. May 2005;54(5):1331-1339.

20. Crute BE, Seefeld K, Gamble J, et al. Functional domains of the alpha1 catalytic subunit of the AMP-activated protein kinase. J Biol Chem. Dec 25 1998;273(52):35347-35354.

21. Cuello F, Bardswell SC, Haworth RS, et al. Protein kinase D selectively targets cardiac troponin $\mathrm{I}$ and regulates myofilament $\mathrm{Ca} 2+$ sensitivity in ventricular myocytes. Circ Res. Mar 30 2007;100(6):864-873.

22. Luo J, Deng ZL, Luo X, et al. A protocol for rapid generation of recombinant adenoviruses using the AdEasy system. Nat Protoc. 2007;2(5):1236-1247. 
23. Volz A, Piper HM, Siegmund B, et al. Longevity of adult ventricular rat heart muscle cells in serum-free primary culture. J Mol Cell Cardiol. Feb 1991;23(2):161-173.

24. Eckel J, van Echten G, Reinauer H. Adult cardiac myocytes in primary culture: cell characteristics and insulin-receptor interaction. Am J Physiol. Aug 1985;249(2 Pt 2):H212-221.

25. Schwenk RW, Dirkx E, Coumans WA, et al. Requirement for distinct vesicle-associated membrane proteins in insulin- and AMP-activated protein kinase (AMPK)-induced translocation of GLUT4 and CD36 in cultured cardiomyocytes. Diabetologia. Oct 2010;53(10):2209-2219.

26. van Oort MM, van Doorn JM, Bonen A, et al. Insulin-induced translocation of CD36 to the plasma membrane is reversible and shows similarity to that of GLUT4. Biochim Biophys Acta. Jan-Feb 2008;1781(1-2):61-71.

27. Alkhateeb H, Chabowski A, Glatz JF, et al. Two phases of palmitate-induced insulin resistance in skeletal muscle: impaired GLUT4 translocation is followed by a reduced GLUT4 intrinsic activity. Am J Physiol Endocrinol Metab. Sep 2007;293(3):E783-793.

28. Bonen A, Luiken JJ, Arumugam Y, et al. Acute regulation of fatty acid uptake involves the cellular redistribution of fatty acid translocase. J Biol Chem. May 12 2000;275(19):1450114508.

29. Luiken JJ, Koonen DP, Willems J, et al. Insulin stimulates long-chain fatty acid utilization by rat cardiac myocytes through cellular redistribution of FAT/CD36. Diabetes. Oct 2002;51(10):3113-3119.

30. Steinbusch LK, Wijnen W, Schwenk RW, et al. Differential regulation of cardiac glucose and fatty acid uptake by endosomal $\mathrm{pH}$ and actin filaments. Am J Physiol Cell Physiol. Jun 2010;298(6):C1549-1559.

31. Bertrand L, Ginion A, Beauloye C, et al. AMPK activation restores the stimulation of glucose uptake in an in vitro model of insulin-resistant cardiomyocytes via the activation of protein kinase B. Am J Physiol Heart Circ Physiol. Jul 2006;291(1):H239-250.

32. Brindley DN, Kok BP, Kienesberger PC, et al. Shedding light on the enigma of myocardial lipotoxicity: the involvement of known and putative regulators of fatty acid storage and mobilization. Am J Physiol Endocrinol Metab. May 2010;298(5):E897-908.

33. Shulman GI. Cellular mechanisms of insulin resistance. J Clin Invest. Jul 2000;106(2):171-176.

34. Steinbusch LK, Schwenk RW, Ouwens DM, et al. Subcellular trafficking of the substrate transporters GLUT4 and CD36 in cardiomyocytes. Cell Mol Life Sci. May 62011.

35. Liu LZ, Cheung SC, Lan LL, et al. Berberine modulates insulin signaling transduction in insulinresistant cells. Mol Cell Endocrinol. Apr 12 2010;317(1-2):148-153.

36. Ginion A, Auquier J, Benton CR, et al. Inhibition of the mTOR/p70S6K pathway is not involved in the insulin-sensitizing effect of AMPK on cardiac glucose uptake. Am J Physiol Heart Circ Physiol. May 202011.

37. Holloway GP, Benton CR, Mullen KL, et al. In obese rat muscle transport of palmitate is increased and is channeled to triacylglycerol storage despite an increase in mitochondrial palmitate oxidation. Am J Physiol Endocrinol Metab. Apr 2009;296(4):E738-747.

38. St-Denis JF, Cabaniols JP, Cushman SW, et al. SNAP-23 participates in SNARE complex assembly in rat adipose cells. Biochem J. Mar 15 1999;338 ( Pt 3):709-715.

39. Bostrom $\mathrm{P}$, Andersson L, Rutberg $\mathrm{M}$, et al. SNARE proteins mediate fusion between cytosolic lipid droplets and are implicated in insulin sensitivity. Nat Cell Biol. Nov 2007;9(11):1286-1293.

40. Bossuyt J, Chang CW, Helmstadter K, et al. Spatiotemporally distinct PKD activation in adult cardiomyocytes in response to phenylephrine and endothelin. J Biol Chem. Jul 272011. 
CHAPTER 7

\section{A Protein Kinase D1-MEF2D Pathway Controls Metabolic Substrate Switching in the Failing Heart}

Ellen Dirkx ${ }^{a}$, Guillaume J.J.M. van Eys ${ }^{a}$, Robert W. Schwenk ${ }^{a}$, Laura K.M. Steinbusch ${ }^{a}$, Nicole Hoebers ${ }^{a}$, Ben J. Janssen ${ }^{b}$, Boudewijn Brans ${ }^{c}$, Andreas T. Vogg ${ }^{d}$, Rhonda Bassel-Duby ${ }^{\text {}}$, Jan F.C. Glatz ${ }^{a}$ and Joost J.F.P. Luiken ${ }^{\text {a }}$

Departments of ${ }^{a}$ Molecular Genetics and ${ }^{\mathrm{b}}$ Pharmacology, Cardiovascular Research Institute Maastricht (CARIM), Maastricht University, the Netherlands,

${ }^{\mathrm{C}}$ Department of Nuclear Medicine, Maastricht University Medical Center, the Netherlands,

${ }^{d}$ Department of Nuclear Medicine, University Hospital Aachen, Germany

e Department of Molecular Biology, University of Texas Southwestern Medical Center,

Dallas, TX 75390-9148, USA 


\begin{abstract}
Background: Disturbances in metabolic substrate uptake promote the development of cardiomyopathy and eventually lead to heart failure. Understanding the pathways that control substrate uptake could provide therapeutic approaches that prevent pathological cardiac remodeling. We studied whether contractionactivated protein kinase D1 (PKD1) regulates metabolic substrate switching in the heart.
\end{abstract}

Methods and Results: In cardiomyocytes from heart-specific PKD1 overexpression (a-MHC-caPKD1) mice glucose uptake was increased, while fatty acid uptake remained unaffected. This substrate switch was confirmed in vivo by microPET imaging, and observed to result in eccentric cardiac hypertrophy and a decreased life span of a-MHC-caPKD1 mice. The transcription factor MEF2D has been implicated in PKD1-mediated hypertrophy. In accordance, in MEF2D knock-out mice, active PKD1 was unable to upregulate GLUT4 gene expression. This indicates that the transciption factor MEF2D not only regulates hypertrophic gene expression but also is part of the PKD1-GLUT4 pathway.

Administation of a high fat diet turned hearts of wild-type mice lipid-overloaded and resulted in concentric cardiac hypertrophy, accompanied by insulin resistance. Strikingly, hearts of a-MHC-caPKD1 mice on a high fat diet displayed neither eccentric nor concentric hypertrophy, and the mice had a normal life span. PET-scans showed that these mice did not increase cardiac glucose uptake or fatty acid uptake, demonstrating a role of PKD1 in balancing cardiac substrate utilization.

Conclusion: In the heart, the PKD1-MEF2D pathway is able to direct metabolic substrate switching (glucose vs. fatty acids). A balanced metabolic substrate uptake is crucial in preventing pathological cardiac remodeling. 


\section{Introduction}

Clinical observations and animal models asseverate that diet contributes to the occurrence and deterioration of cardiomyopathy. Pathological cardiac hypertrophy can be caused by a number of stress conditions such as high blood pressure, diabetes and obesity. Initially hypertrophy can be compensatory or adaptive but, when sustained over time, it often becomes maladaptive and leads to heart failure. Metabolic changes may play an essential role in the development of such hypertrophic cardiomyopathy. ${ }^{1}$ In particular, increased supply of long chain fatty acids (LCFA) and utilization by the heart results in the development of cardiac insulin resistance and diabetic cardiomyopathy, of which hypertrophy is a hallmark. ${ }^{2}$

Substrate utilization in the healthy heart is predominantly regulated by GLUT4 and CD36, the main cardiac transporters for glucose and LCFA, respectively. ${ }^{3}$ Both GLUT4 and CD36 are stored in endosomal compartments, from where these transporters can translocate to the sarcolemma. GLUT4 and CD36 translocation to the sarcolemma responds to particular physiological stimuli, such as increased insulin levels and elevated contractile activity. ${ }^{3-5}$ The diabetic heart, which is characterized by insulin resistance, increases LCFA utilization at the expense of glucose. The greater influx of LCFA is due to a permanent CD36 relocation from intracellular stores to the sarcolemma, ${ }^{3}$ and has been shown to exceed the mitochondrial $\beta$ oxidative capacity. ${ }^{6}$ Preference of LCFA uptake and fatty acid oxidation results in a gradual build-up of inert triacylglycerol (TAG) stores, and an increase in bioactive lipid metabolites diacylglycerols (DAG) and ceramides inside the cardiomyocytes. These events cause an accumulation of reactive oxygen species (ROS) in the heart. Furthermore, the lipid compounds activate Ser/Thr-kinase cascades which impair upstream insulin signaling, and subsequently reduce insulin-stimulated glucose uptake. $^{7}$ Over time, both excessive lipid accumulation and progressive insulin resistance will gradually initiate a hypertrophic remodeling process in the heart. ${ }^{8}$

Exercise stimulates glucose uptake in the type-2 diabetic heart, and diminishes cardiac remodeling. ${ }^{9}$ Therefore, contraction signaling may activate alternative mechanisms that repair defective insulin-induced GLUT4 translocation. ${ }^{7}$ Previously, we showed that contraction induces activation of protein kinase D1 (PKD1), which leads to increased GLUT4-mediated glucose uptake by cardiomyocytes. ${ }^{10}$ Thus, PKD1 activation may restore cardiac glucose utilization in the insulin resistant heart and prevent cardiac remodeling. However, besides its effect in metabolism, increased expression of PKD1 has also been shown to result in dilated cardiac hypertrophy and heart failure. ${ }^{11}$ A possible mechanism to explain the effect of PKD1 on hypertrophic remodeling is via HDAC5 shuttling. PKD1-induced phosphorylation of HDAC5 leads to its removal from the nucleus. ${ }^{12}$ In the non-phosphorylated state, HDAC5 is retained in the nucleus where it associates and inhibits myocyte enhancer factor-2 (MEF2), preventing activation of a number of genes involved in cardiac hypertrophy. However, upon phosphorylation, HDAC5 is exported to the cytoplasm and can no 
longer bind to MEF2, so that MEF2 is able to activate hypertrophic genes. Thus, phosphorylation of HDAC5 by an activated PKD1 will promote cardiac hypertrophy.

The aim of this study was to explore a potential role for PKD1 in controlling cardiac substrate uptake and hypertrophic remodeling of the heart. First, by using isolated cardiomyocytes, we demonstrated a role for PKD1 in metabolic substrate shifting in vitro. Then, we extended this study in vivo using different mouse models. Our findings show that PKD1 links alterations in metabolism to cardiac hypertrophy. PKD1 overexpression in the healthy heart is unfavorable because it leads, via activation of MEF2D, to increased expression of both GLUT4 and hypertrophic genes. The resulting eccentric hypertrophy can be prevented by a high fat diet, which paradoxically rescues the heart from hypertrophic remodeling and does not induce cardiac insulin resistance. This indicates that a balanced substrate utilization is crucial for proper cardiac performance and that PKD1 is essential for maintaining this balance. 


\section{Materials and Methods}

\section{Animals}

Cardiac-specific constitutively active PKD1 overexpression ( $\alpha$ MHC-caPKD1) mice have been described by Harrison et al. ${ }^{13}$ Myocyte enhancer factor-2D (MEF2D) knock-out (KO) mice have been described by Kim et al. ${ }^{14}$ From the age of 12 weeks, the $\alpha M H C-c a P K D 1$ mice were administered a low fat diet containing 10 en\% fat (diet number: D12450B, Research Diets, New Brunswick, NJ, USA) or a high fat diet containing 45 en\% fat (diet number: D12451, Research Diets, New Brunswick, NJ, USA). Animals were maintained at the Experimental Animal Facility of Maastricht University. The investigation conforms to the Guide for the Care and Use of Laboratory Animals published by the US National Institutes of Health (NIH Publication No. 85-23, revised 1996).

\section{Materials}

Antibodies Phospho-PKD/PKC- $\mu$ (Ser916) was obtained from Cell Signaling (Beverly, USA). The antibody directed against GLUT4 was obtained from Santa Cruz Biotechnology (Santa Cruz, USA) and that against mouse CD36 was from Chemicon (Billerica, USA).

Chemicals Liberase blendzyme 1 was obtained from Roche Diagnostics (Indianapolis, IN). ). All culture dishes, pipets, tubes and containers used, were from Greiner Bio-One (Alphen aan de Rijn, the Netherlands).

\section{Isolation and treatment of adult mouse cardiomyocytes}

Cardiomyocytes were isolated from male and female WT- or transgenic C57BL/6 mice using a Langendorff-perfusion-system as previously described, ${ }^{13}$ and were subjected to electric field stimulation (EFS) for 4 minutes at $37^{\circ} \mathrm{C}(2 \mathrm{~Hz}, 40 \mathrm{~V}$, pulse duration $10 \mathrm{msec}$ ) or incubated with $100 \mathrm{nmol} / \mathrm{l}$ insulin for $15 \mathrm{~min}$.

\section{Transfection and culturing of adult mouse cardiomyocytes}

Isolated cardiomyocytes from both WT and MEF2D KO mice were cultured in medium 199 (containing 1\% PENSTREP and 1\% FBS) and transfected with PKD adenovirus ( $4 \mu \mathrm{l} / 6$ well). The PKD adenovirus was a kind gift from Prof. Dr. Metin Avkiran. After 15 hours, cells were lysed and RNA was isolated using TRI reagent (Applied Biosystems/Ambion, Austin, USA). 


\section{cDNA synthesis and RT-PCR}

cDNA was synthesized using SuperScript ${ }^{\circledR}$ III Reverse Transcriptase (Invitrogen, Carlsbad, USA) according to the protocol of the manufacturer. GLUT4 gene expression was measured using RT-PCR. Following primers for GLUT4 were used: GCTTTGTGGCCTTCTTTGAG (forward) and CAGGAGGACGGCAAATAGAA (reverse).

\section{Immunoblotting}

Proteins were separated by SDS-PAGE on 4-12\% Bis-Tris Criterion XT precast gels (Bio-rad, California, USA), and transferred to nitrocellulose membranes for Western blotting. The protein bands were visualized as previously described. ${ }^{10}$

\section{Measuring blood glucose and insulin levels}

After 4 hours fasting, blood was collected via the tail vein. To measure blood glucose levels, a blood glucose monitor was used (OneTouch Ultra, Milpitas, CA, USA). Insulin plasma levels were measured by performing an insulin ELISA (Millipore, Billerica, MA, USA).

\section{Intramyocardial triacylglycerol}

Intramyocardial lipids were extracted from snap frozen cardiomyocytes and separated by high-performance thin layer chromatography. ${ }^{14}$ Bands were resolved with a hexane/diethylether/propanol (87:10:3) solution stained with $\mathrm{MnC}_{12} / \mathrm{H}_{2} \mathrm{SO}_{4}$ in methanol. Triacylglycerol bands were detected with a Molecular Imager (ChemiDoc XRS, Bio-rad, California, USA) and analyzed with Quantity One (Bio-rad, California, USA).

\section{Echocardiography}

Transthoracic echocardiographic measurements were performed under 1-2\% isoflurane anaesthesia using a RMV707B (15-45 MHz) scan-head interfaced with a Vevo-770 high frequency ultrasound system (VisualSonics Inc., Toronto, Canada). Long-axis EKG-triggered cine loops of the LV contraction cycle were obtained in Bmode to assess end-diastolic/systolic volume. Short-axis recordings of the LV contraction cycle were taken in M-mode to assess wall thickness of the anterior/posterior wall at the mid-papillary level. From B-mode recordings, LV length from basis to apex, LV internal diameter in systole (LVIDs) and diastole (LVIDd) were determined, and the ejection fraction was calculated from the latter two parameters. 
From M-mode recordings, LV anterior wall thickness in systole (AWths) and diastole (AWthd) were determined.

\section{Heamatoxylin-eosin staining}

Morphology of the heart was visualized using heamatoxylin-eosin staining of paraffin sections from both WT and caPKD mice on a low fat or high fat diet.

\section{Micro-positron emission tomography}

Mice were scanned with a microPET system (Siemens Focus 120, Erlangen, Germany) for imaging studies. After 3 hours fasting, the mice received intraperitoneal injections of $\left[{ }^{18} \mathrm{~F}\right]$-fluoro-deoxyglucose (FDG) or intravenous injections of $14(\mathrm{R}, \mathrm{S})$ $\left[{ }^{18}\right.$ F]fluoro-6-thia-heptadecanoic acid (FTHA). One hour later, a $30 \mathrm{~min}$ image acquisition was performed. During acquisition, mice were maintained under $2 \%$ isoflurane anesthesia. Cardiac $\left[{ }^{18} \mathrm{~F}\right]-\mathrm{FDG}$ and $\left[{ }^{18} \mathrm{~F}\right]-\mathrm{FTHA}$ uptake (expressed as a percentage of the injected dose) was corrected for body weight. Image acquisition for FDG and FTHA were performed on the same mice with a seven days interval.

\section{Statistics}

All data are presented as means \pm SEM. Statistical analysis was performed by using Student's t-test or ANOVA (including Newman-Keuls Multiple Comparison Test) and statistical analysis software Prism 4 (GraphPad Software, Inc.). P<0.05 indicates a statistical significance. 


\section{Results}

\section{PKD1 activation selectively induces glucose uptake}

Substrate uptake was measured in isolated cardiomyocytes from cardiac-specific constitutively active PKD1 overexpression ${ }^{11}$ ( $\alpha$ MHC-caPKD1) and wild type (WT) mice. PKD1 overexpression in these cells resulted in a markedly increased basal and contraction-induced glucose uptake compared to WT cardiomyocytes, whereas basal and contraction-induced long chain fatty acid (LCFA) uptake was not altered (Fig. 1A). Following these in vitro experiments, in vivo microPET imaging of $\alpha \mathrm{MHC}$ caPKD1 mice was performed. Injection of $\left[{ }^{18} \mathrm{~F}\right]$-fluoro-deoxyglucose (FDG) showed a higher level of cardiac glucose uptake in $\alpha$ MHC-caPKD1 mice compared to WT mice (Fig. 1B). These data demonstrate that PKD1 activation selectively induces glucose uptake, but not LCFA uptake.

\section{Figure 1}

A
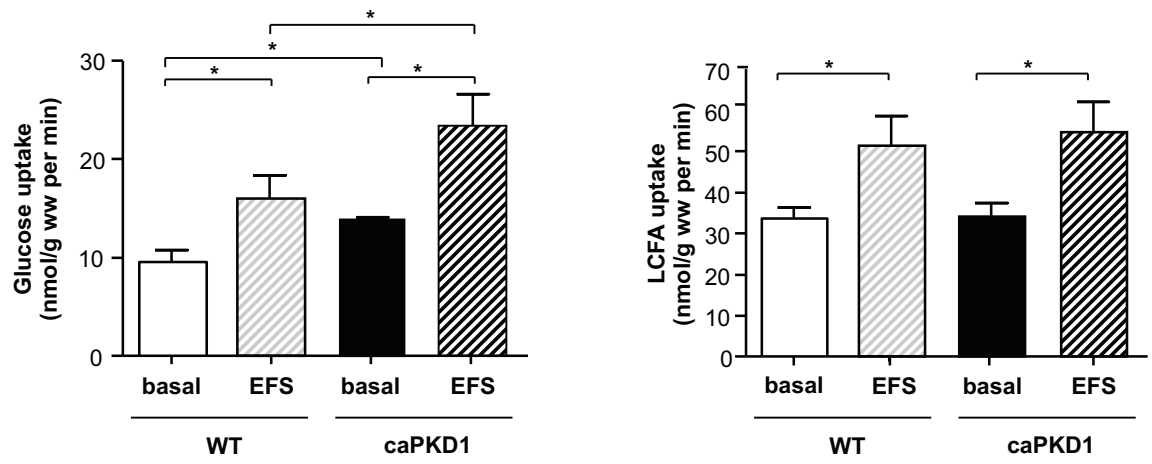

B
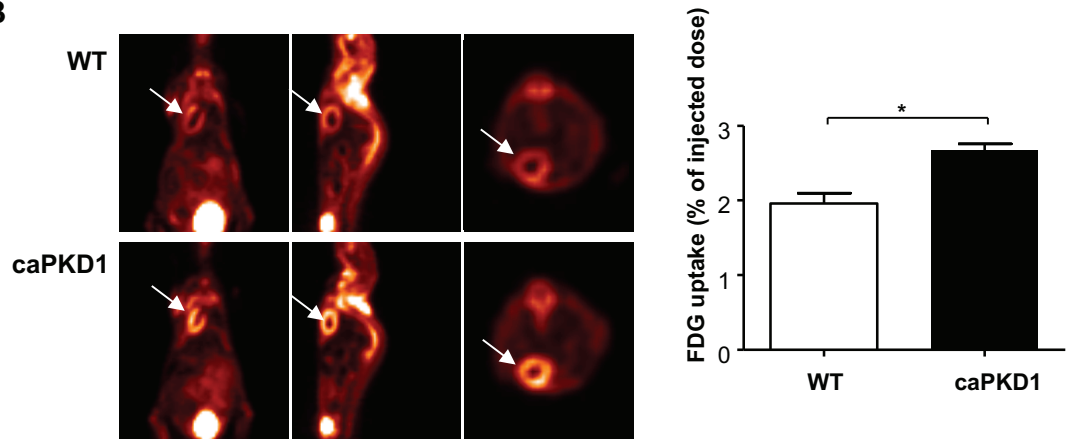

Figure 1. PKD1 is essential for glucose uptake but not for LCFA uptake

(A) Primary cardiomyocytes from WT and caPKD1-mice were electric field stimulated (EFS), and subsequently used for measurement of $\left[{ }^{14} \mathrm{C}\right]$ palmitate and $\left[{ }^{3} \mathrm{H}\right]$ glucose uptake $(n=6)$.. (B) WT and $\alpha \mathrm{MHC}$ - 
caPKD1 mice were injected with $\left[{ }^{18} \mathrm{~F}\right]$ FDG followed after $1 \mathrm{~h}$, by 30 min microPET scanning $(\mathrm{n}=4)$. Cardiac uptake of $\left[{ }^{18} \mathrm{~F}\right] F D G$ in both WT and $\alpha$ MHC-caPKD1 mice was visualized (left panels). Representative images show coronal, sagittal and transverse view of a WT and $\alpha$ MHC-caPKD1 mouse. The arrows indicate the heart. Images were quantified by percentage of injected dose (right panel). * Statistically different $(p<0.05)$.

\section{Effect of high fat diet on lifespan of WT and $\alpha$ MHC-caPKD1 mice}

Balanced substrate uptake favors proper cardiac function. Since $\alpha \mathrm{MHC}$-caPKD1 mice have an increased glucose uptake, we hypothesize that administering a high fat diet may increase fatty acid uptake and restore the cardiac substrate. Therefore, both WT mice and $\alpha$ MHC-caPKD1 mice were subjected to a high fat diet for 20-weeks. In WT mice, as in $\alpha$ MHC-caPKD1 mice, the high-fat diet regime increases body mass, blood glucose, and plasma insulin (Fig. 2A-C), confirming the effectiveness of high fat diet in inducing obesity and whole-body insulin resistance. When comparing the life span of these mice, the high fat diet had no effect on the survival of the WT mice. They showed the same survival proportion whether on a low fat or a high fat diet (Fig. 2D). The $\alpha$ MHC-caPKD1 mice on a low fat diet died much faster than the WT groups. However, when these mice were fed a high fat diet, the life span of $\alpha M H C-c a P K D 1$ mice was drastically prolonged, and was approximately the same as the WT mice on a low fat diet (Fig. 2D). Thus, the high fat diet prevented shortening of the lifespan in $\alpha$ MHC-caPKD1 mice.

\section{Figure 2}

A

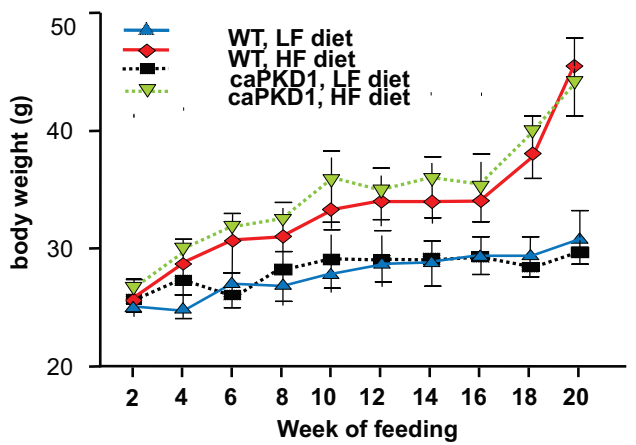


B

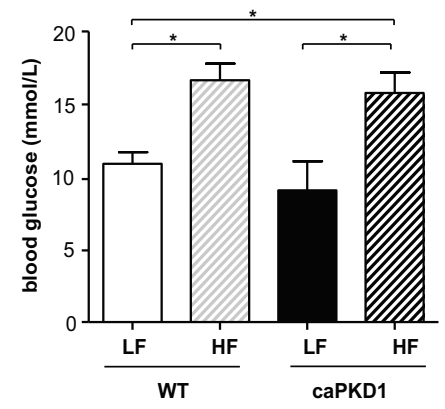

C

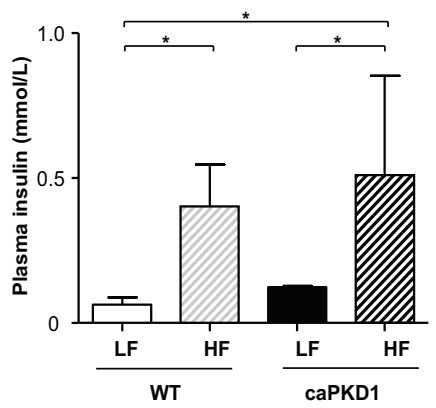

D

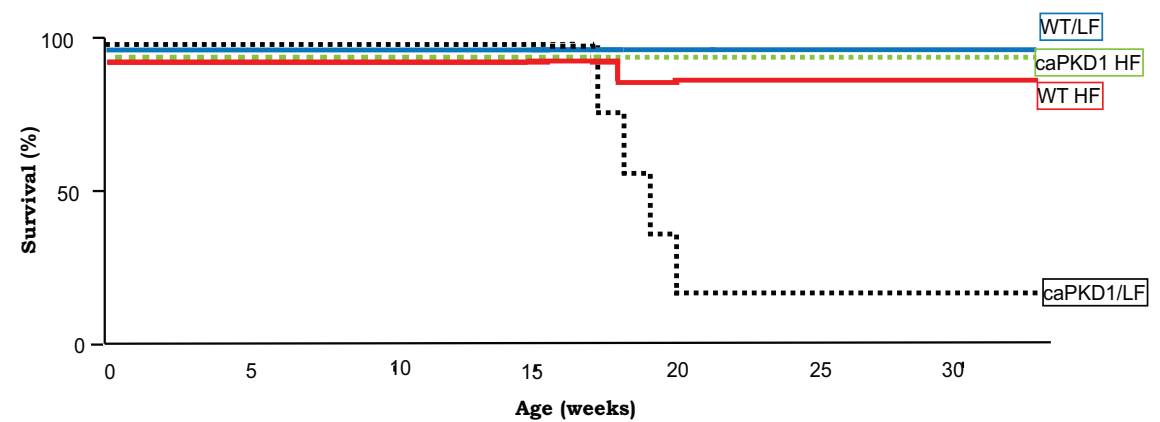

Figure 2. High-fat diet administration to WT and $\alpha M H C$-caPKD1 mice induces whole-body insulin resistance

After 20 weeks of high-fat diet-feeding, (A) body mass, as well as (B) fasted blood glucose levels and (C) fasted plasma insulin levels were determined. * Statistically different $(p<0.05)$. (D) Life span was shortened in $\alpha$ MHC-caPKD1 mice on a low-fat diet. WT and $\alpha$ MHC-caPKD1 mice were subjected to high-fat diet for up to 20 weeks, and scored for survival (\%). Amount of animals at the start of the experiment: WT LF ( $n=13)$, WT HF ( $n=14)$, caPKD1 LF ( $n=12)$, caPKD1 HF ( $n=11)$.

\section{$\alpha$ MHC-caPKD1 mice are protected from high-fat diet induced insulin re- sistance}

High fat diet is known to cause decreased cardiac insulin sensitivity. Therefore, it was tested whether cardiac insulin sensitivity was decreased in mice on a high fat diet. In the heart of WT mice, high fat diet led to an increased intramyocardial triacyl glycerol (TAG) content (Fig. 3A). In insulin-stimulated cardiomyocytes derived from WT mice on a high fat diet, phosphorylation of markers for insulin sensitivity, Akt-Ser473 and GSK3 $\beta$-Ser9, were markedly reduced compared to cardiomyocytes from WT mice on a low fat diet (Fig. 3B). Consistently, insulin-stimulated glucose and LCFA uptake in cardiomyocytes from high fat diet fed WT mice were severely reduced (Fig. 3C, D), and basal cardiac LCFA uptake was increased (Fig. 3D). In contrast, no increased intramyocardial lipid levels or increased basal LCFA uptake levels 
were observed in $\alpha M H C-c a P K D 1$ mice on a high fat diet (Fig 3D). Hearts from $\alpha M H C-c a P K D 1$ mice on a low fat diet did not show significantly different intramyocardial lipid levels compared to $\alpha$ MHC-caPKD1 mice on a high fat diet (Fig 3D). As for insulin sensitivity, cardiomyocytes from $\alpha$ MHC-caPKD1 mice displayed the same level of insulin-stimulated Akt-Ser473 phosphorylation and GSK3 $\beta$-Ser9 phosphorylation as those from WT mice on a low fat diet (Fig. 3B). Accordingly, cardiomyocytes from high fat diet fed $\alpha$ MHC-caPKD1 mice retained full insulin-induced responses on substrate uptake: i.e., insulin-stimulated glucose and LCFA uptake were not significantly different from cardiomyocytes of WT mice on a low fat diet (Fig. 3B, $C, D)$. Thus, high fat diet caused cardiac insulin resistance in WT mice but not in $\alpha \mathrm{MHC}$-caPKD1 mice.

Figure 3

A

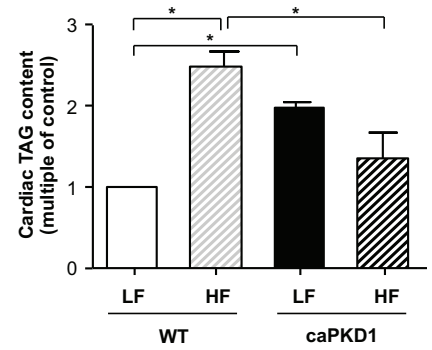

Figure 3. Hearts from $\alpha M H C-c a P K D 1$ mice are protected against high-fat diet-induced lipid accumulation and insulin resistance

(A) At the end of a period of 20 weeks diet, mice were sacrificed for isolation of primary cardiomyocytes from WT and QMHC-caPKD1 mice on low-fat and high-fat diet for determination of intracellular TAG content $(n=5)$. Primary cardiomyocytes from WT and $\alpha$ MHC-caPKD1 mice on low fat and high fat diet were treated with or without insulin (ins; $100 \mathrm{nM} ; 15 \mathrm{~min}$ ), and subsequently used for (B) Western detection of Akt-Ser473- and GSK3 $\alpha / \beta$-Ser9 phosphorylation, and (C) for uptake of $\left[{ }^{3} \mathrm{H}\right]$ deoxyglucose and (D) $\left[{ }^{14} \mathrm{C}\right]$ palmitate $(n=4)$. For both glucose and LCFA uptake, the insulin-stimulated component was calculated (ins-basal) and is displayed in the corresponding right panels $(n=4)$. * Statistically different $(p<0.05)$. 
B
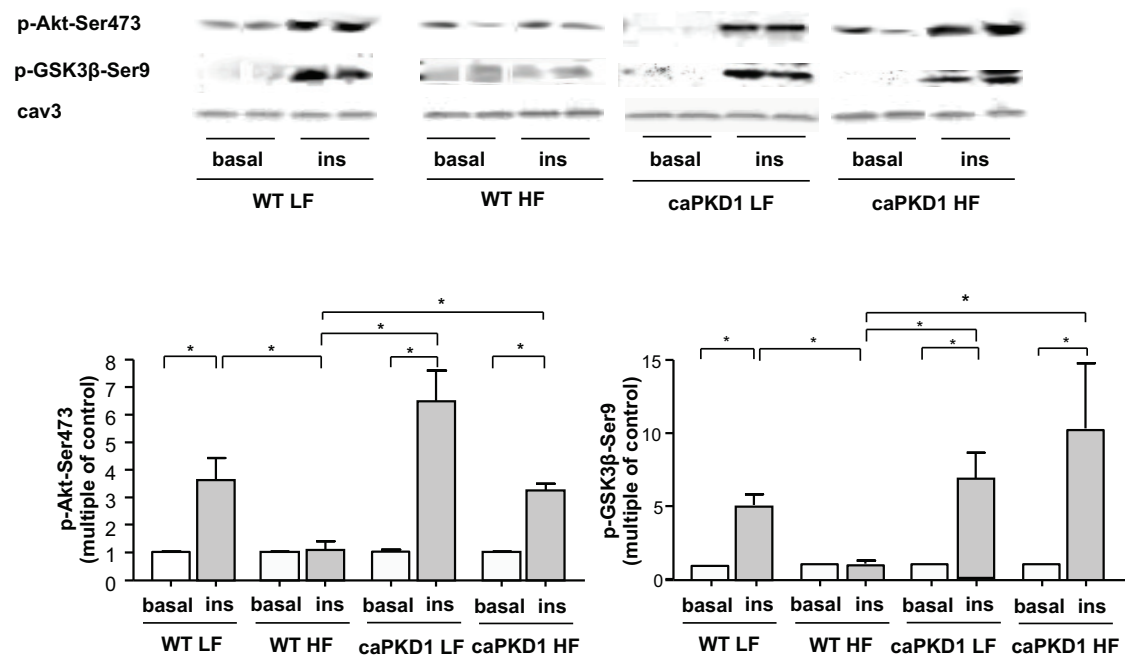

C

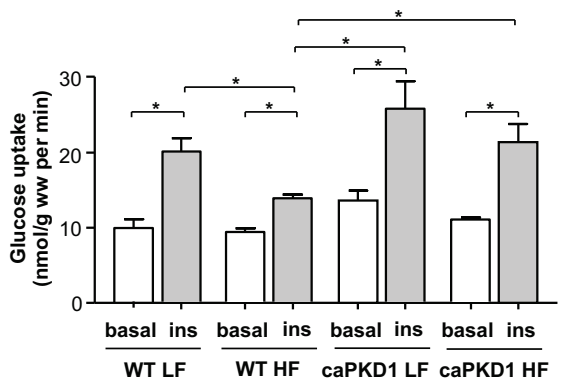

Insulin-stimulated component of

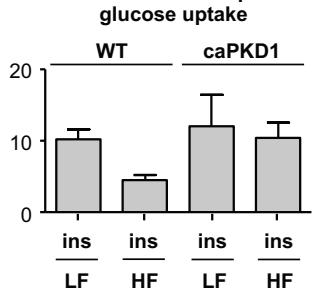

D
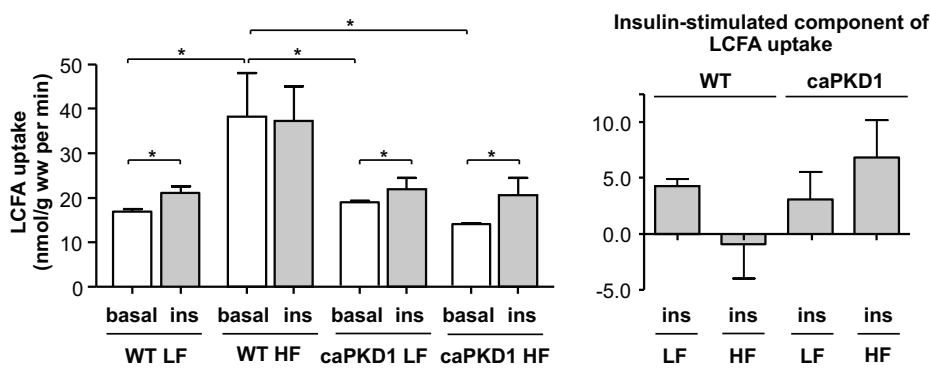

\section{A high fat diet balances cardiac substrate uptake in $\alpha$ MHC-caPKD1 mice}

Cardiac substrate uptake was measured in vivo by microPET imaging. WT and $\alpha M H C-c a P K D 1$ mice on a low fat diet or high fat diet for 20 weeks were injected 
with $\left[{ }^{18} \mathrm{~F}\right]-\mathrm{FDG}$ to measure glucose uptake or with $\left[{ }^{18} \mathrm{~F}\right]$-FTHA to measure LCFA uptake. WT mice on a high fat diet displayed an increase in cardiac $\left[{ }^{18} \mathrm{~F}\right]-\mathrm{FTHA}$ uptake compared to WT mice on a low fat diet (Fig. 4A), whereas $\left[{ }^{18} \mathrm{~F}\right]$-FDG uptake was not significantly changed (although there seems to be a trend towards decreased uptake) (Fig. 4B). In $\alpha$ MHC-caPKD1 mice on a high fat diet, no such increase of cardiac $\left[{ }^{18} \mathrm{~F}\right]$-FTHA uptake was detected (Fig. 4A). In $\alpha$ MHC-caPKD1 mice on a low fat diet, cardiac $\left[{ }^{18} \mathrm{~F}\right]-\mathrm{FDG}$ levels were increased and $\left[{ }^{18} \mathrm{~F}\right]-\mathrm{FTHA}$ levels were decreased compared to WT mice on a low fat diet. However, in $\alpha$ MHC-caPKD1 mice on a high fat diet, cardiac $\left[{ }^{18} \mathrm{~F}\right]-$ FDG and $\left[{ }^{18} \mathrm{~F}\right]-\mathrm{FTHA}$ uptake levels were comparable to those in WT mice on a low fat diet (Fig. 4B). Interestingly, consistent with an increase in glucose uptake, cardiac GLUT4 protein expression was increased in $\alpha$ MHC-caPKD1 mice on a low fat diet Fig. $4 C$ ). Administration of a high fat diet to $\alpha$ MHC-caPKD1 mice resulted in a decrease in cardiac GLUT4 expression levels. In addition, WT mice on a high fat diet exhibited a decrease in GLUT4 expression levels compared to WT mice on a low fat diet. These data demonstrate that high fat diet administration balances cardiac substrate uptake in $\alpha$ MHC-caPKD1 mice.

\section{Figure 4}

A
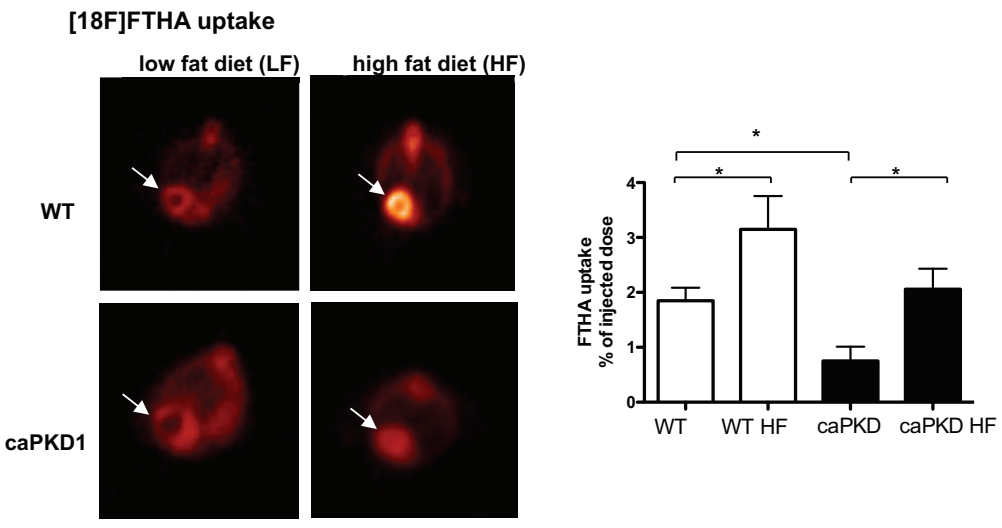
B [18F]FDG uptake

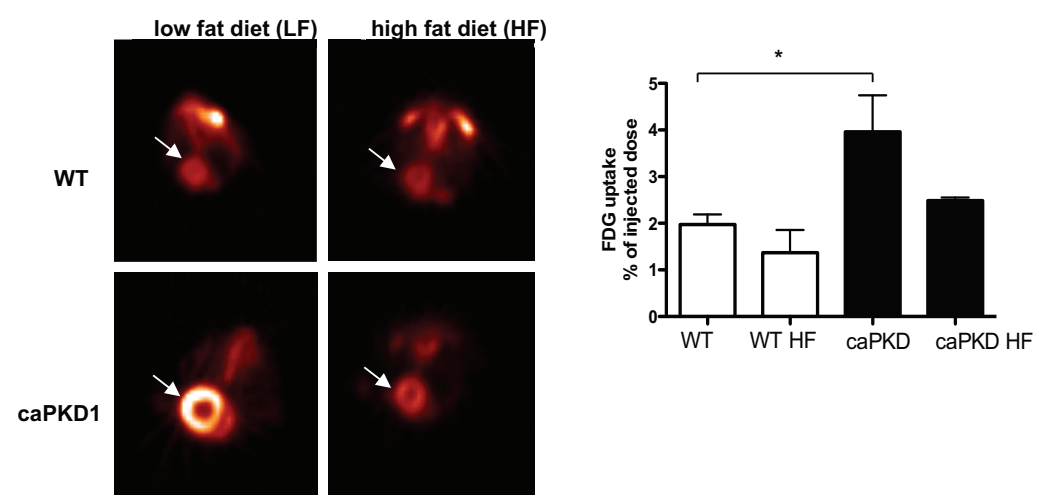

C

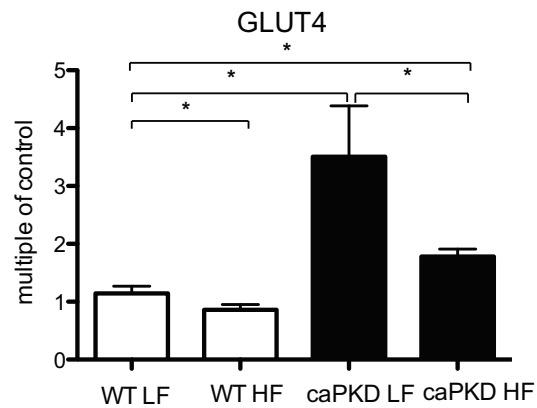

Figure 4. Substrate uptake in $\alpha M H C-c a P K D 1$ mice on a high fat diet is balanced By using microPET imaging, in vivo glucose and LCFA uptake were measured in WT and aMHC-caPKD1 mice on a low fat (LF) or a high fat (HF) diet. WT and $\alpha$ MHC-caPKD1 mice were injected with $\left[{ }^{18}\right.$ F]FDG and $\left[{ }^{18}\right.$ F]FTHA, followed after $1 \mathrm{~h}$ by 30 min microPET scanning $(n=4)$. Representative images show the cardiac uptake of (A) $\left[{ }^{18} \mathrm{~F}\right] \mathrm{FTHA}$ and (B) $\left[{ }^{18} \mathrm{~F}\right]$ FDG in both WT and $\alpha \mathrm{MHC}$-caPKD1 mice (left panel, arrows indicate the heart). Collected images were quantified as percentage of injected dose (right panel). (C) GLUT4 protein expression was measured in WT and aMHC-caPKD1 mice on a low fat or high fat diet. * Statistically different $(p<0.05)$.

\section{A high fat diet normalizes cardiac morphology and function in $\alpha$ MHC- caPKD1 mice}

The effect of high fat diet feeding on cardiac morphology and function was assessed by echocardiography. The hearts of mice on a high fat diet displayed decreased left ventricle internal diameters (Fig. 5A, B) and left ventricular length from basis to apex (Fig. 5C), whereas concomitantly, ejection fraction (Fig. 5C) and anterior wall thickness (Fig. 5D) increased. These physiological changes coincide with concentric hypertrophic remodeling (Fig. $5 E$ ). Hearts from $\alpha M H C$-caPKD1 mice on a low fat diet show eccentric cardiac hypertrophy (Fig. 5A, E) and decreased cardiac function (Fig. 5B-D). These alterations of cardiac structure and function, seen in $\alpha$ MHC-caPKD1 mice on a low fat diet, were consistent with previously reported dilated cardiomyo- 
pathy ${ }^{11}$. On the other hand, cardiac morphology and function in $\alpha$ MHC-caPKD1 mice on a high fat diet were similar to those of WT mice on a low fat diet (Fig. 4, Table 1). No concentric or dilated hypertrophic remodeling was observed (Fig. 5A, E), and ejection fraction was not decreased (Fig. 5C). These findings show a role for 1 ) cardiac overexpression of PKD1 in preventing high fat diet-induced cardiac hypertrophy and 2) high fat diet in preventing cardiac hypertrophy in $\alpha$ MHC-caPKD1 mice. These data link increased cardiac glucose uptake in $\alpha$ MHC-caPKD1 mice to dilated cardiac hypertrophy. However, cardiac morphology and function can be normalized by feeding $\alpha \mathrm{MHC}$-caPKD1 mice a high fat diet.

\section{Figure 5}

A

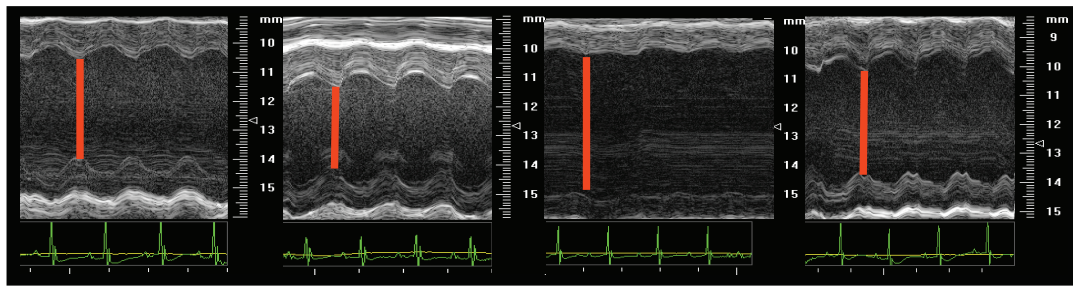

WT LF

B
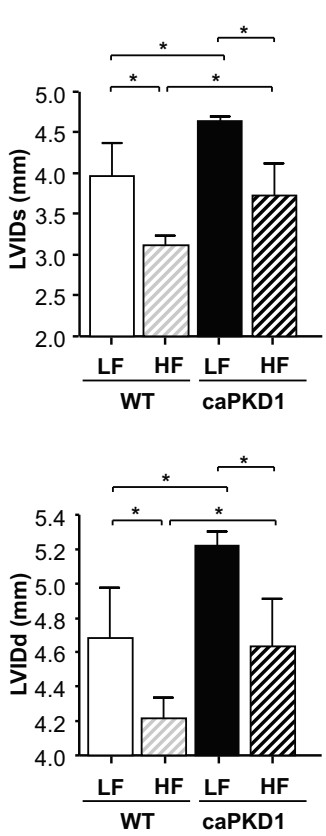

C
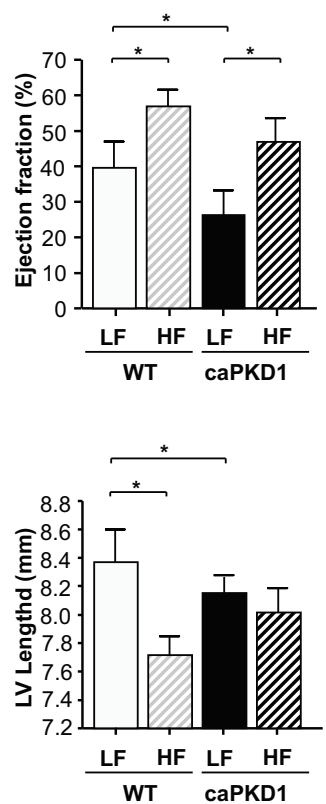

D
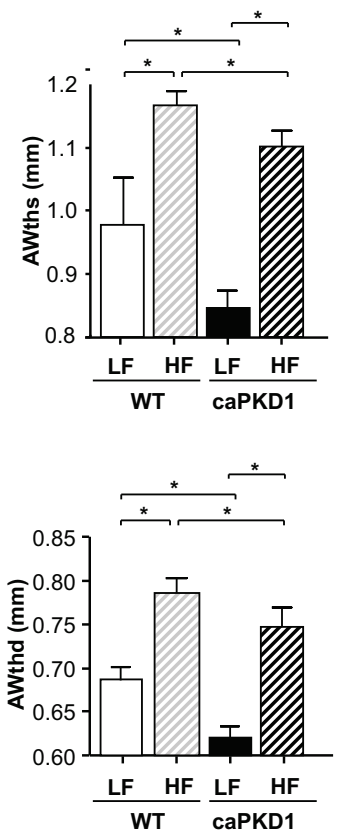


\section{E}

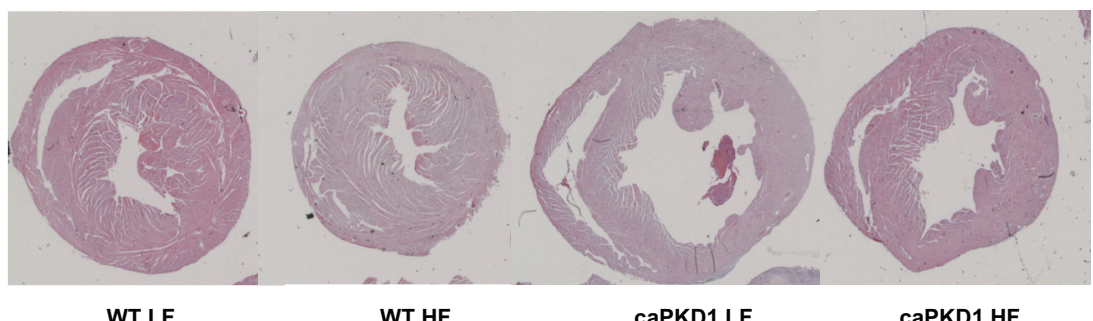

Figure 5. Effects of cardiac PKD1 overexpression and high-fat diet on cardiac morphological parameters WT and $\alpha$ MHC-caPKD1 mice on low-fat (LF) and high-fat (HF) diet were subjected to echocardiography measurements after 20 weeks of diet. (A) From M-mode recordings, (B) LV anterior wall thickening in systole (AWths) and diastole (AWthd) were determined. (C) From B-mode recordings LV internal diameter in systole (LVIDs) and diastole (LVIDd) were determined, and (D) ejection fraction was calculated from these parameters. In these same recordings, (D) LV length from basis to apex was determined. Values represent averages of following animal groups: WT LF $(n=9)$, WT HF $(n=8)$, caPKD1 LF $(n=4)$, caPKD1 HF $(n=8)$. Representative M-mode images are displayed. * Statistically different $(p<0.05)$. (E) The morphology of the hearts of both WT and QMHC-caPKD1 mice on a LF or HF diet were visualized with a heamatoxylin-eosin staining of the heart. Representative images were shown.

\section{Table 1}

Echocardiographic characterization of WT versus caPKD1 mice

\begin{tabular}{|c|c|c|c|c|}
\hline \multirow{3}{*}{ Characteristic } & \multicolumn{4}{|c|}{ Results by type and ( $n$ ) of mouse } \\
\hline & \multicolumn{2}{|r|}{ Wild type } & \multicolumn{2}{|c|}{ caPKD1 } \\
\hline & LF diet (9) & HF diet (8) & LF diet (4) & HF diet (8) \\
\hline HR (bpm) & $448 \pm 13$ & $439 \pm 21$ & $421 \pm 15$ & $421 \pm 20$ \\
\hline AWths $(\mathrm{mm})$ & $0.97 \pm 0.07$ & $1.17 \pm 0.03^{*}$ & $0.86 \pm 0.02^{* * *}$ & $1.12 \pm 0.05$ \\
\hline AWthd (mm) & $0.69 \pm 0.03$ & $0.79 \pm 0.02^{*}$ & $0.62 \pm 0.01^{* * *}$ & $0.74 \pm 0.02^{* *}$ \\
\hline PWths (mm) & $0.94 \pm 0.07$ & $1.11 \pm 0.02^{*}$ & $0.80 \pm 0.03^{* * *}$ & $1.06 \pm 0.05$ \\
\hline PWthd (mm) & $0.73 \pm 0.04$ & $0.77 \pm 0.02$ & $0.61 \pm 0.01^{* * *}$ & $0.74 \pm 0.02$ \\
\hline LVIDs (mm) & $3.97 \pm 0.4$ & $3.04 \pm 0.11^{*}$ & $4.60 \pm 0.30^{* * *}$ & $3.72 \pm 0.39^{* *}$ \\
\hline LVIDd (mm) & $4.68 \pm 0.29$ & $4.15 \pm 0.12^{*}$ & $5.21 \pm 0.20^{* * *}$ & $4.63 \pm 0.28^{* *}$ \\
\hline$E S V \_L(m m 3)$ & $0.41 \pm 0.10$ & $0.22 \pm 0.04^{*}$ & $0.55 \pm 0.03^{* * *}$ & $0.38 \pm 0.12^{* *}$ \\
\hline EDV_L (mm3) & $0.63 \pm 0.09$ & $0.50 \pm 0.04^{*}$ & $0.72 \pm 0.02$ & $0.66 \pm 0.11^{* *}$ \\
\hline LVLs_apic $(\mathrm{mm})$ & $7.77 \pm 0.28$ & $7.26 \pm 0.16^{*}$ & $7.78 \pm 0.23$ & $7.70 \pm 0.24^{* *}$ \\
\hline LVLd apic (mm) & $8.37 \pm 0.23$ & $7.71 \pm 0.14^{*}$ & $8.10 \pm 0.23$ & $8.21 \pm 0.14^{* *}$ \\
\hline$\% \mathrm{FS}$ & $21.4 \pm 3.40$ & $26.8 \pm 1.52^{*}$ & $13.1 \pm 0.86^{* * *}$ & $20.7 \pm 3.66^{* *}$ \\
\hline
\end{tabular}

* WT on a low fat ( LF) diet versus WT on a high fat (HF) diet

** caPKD1 on a HF diet versus WT on a HF diet

*** WT on a LF diet versus caPKD1 on a LF diet

Table 1: HR; heart rate, AWth; LV anterior wall thickening, LVID; LV internal diameter, ESV_L; end systolic volume of the left ventricle, EDV-L; end diastolic volume of the left ventricle, \% FS; \% of fractional shortening 


\section{How does PKD1 link cardiac glucose uptake to hypertrophic remodeling?}

To establish a link between PKD1-induced increase in glucose uptake and PKD1induced hypertrophy, we tested the hypothesis whether, in addition to stimulation of hypertrophy, PKD1 also increases GLUT4-mediated glucose uptake via the HDAC5-MEF2 pathway. It has been demonstrated that MEF2D, one of the mayor MEF2 isoforms in the heart, mediates stress-dependent cardiac remodeling. ${ }^{14}$ Because also PKD1 has been shown to mediate stress-dependent cardiac hypertrophy, $\underline{13}$ we tested whether MEF2D functions downstream of PKD1 in a pathway that stimulates GLUT4 gene expression. Overexpression of PKD1 in cardiomyocytes from WT mice showed increased GLUT4 gene expression (Fig. 6A). In MEF2D KO cardiomyocytes, basal GLUT4 gene expression was significantly decreased and, importantly, overexpression of PKD1 did not result in up-regulation of GLUT4 (Fig. 6A). These data indicate that PKD1 is capable to up-regulate GLUT4 gene expression via activation of MEF2D.

To clarify how a high fat diet can rescue $\alpha$ MHC-caPKD1 mice from developing a pathological cardiomyopathy, we measured PKD1 protein expression in the hearts of WT and $\alpha M H C-c a P K D 1$ mice on a low fat and high fat diet. In both WT and $\alpha$ MHC-caPKD1 mice on a high fat diet, cardiac PKD1 protein expression was downregulated (Fig. 6B). Thus, the high fat diet was able to repress PKD1 expression and thereby neutralized PKD1-mediated effects on cardiac function.

\section{Figure 6}

A

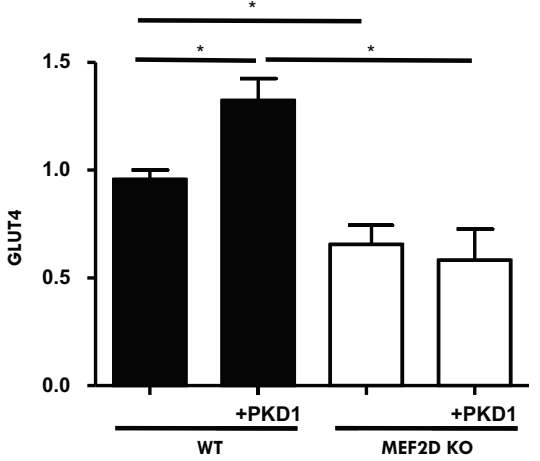

B

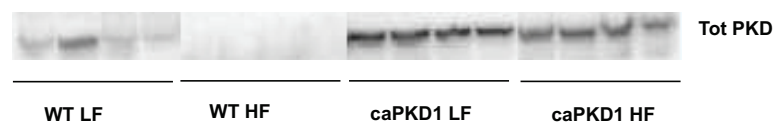

Figure 6. PKD increases GLUT4 gene expression via MEF2D activation

(A) Primary cardiomyocytes were isolated from WT and MEF2D KO mice. After culturing and transfecting the cardiomyocytes with PKD1 adenovirus (for 15 hours), cells were lysed and GLUT4 gene expression was measured. (B) Primary cardiomyocytes from WT and $\alpha$ MHC-caPKD1 mice on low fat and high fat 
diet were isolated and subsequently used for Western detection of total PKD1 protein expression. * Statistically different $(p<0.05)$.

\section{DISCUSSION}

Cardiomyopathy accompanied with type 2 diabetes or obesity is often the result of prolonged periods of increased lipid uptake as a consequence of a high fat $\operatorname{diet}^{2}$. A change in substrate uptake towards increased glucose utilization could contribute to a reduction of heart failure risk in diabetic and obese patients.

Our experiments show that PKD1 induces GLUT4 gene expression, which leads to increased translocation of this transporter to the plasma membrane ${ }^{10}$ and eventually increased glucose uptake. Furthermore, PKD1 does not increase LCFA uptake, which makes it an ideal candidate to selectively increase glucose uptake in the heart. Therefore, we tested whether high PKD1 expression mitigates the negative consequences of a high fat diet and subsequent insulin resistance in the heart. To that end, we employed transgenic mice that overexpress PKD1 in the heart ( $\alpha$ MHCcaPKD1) and fed them different diets. On a low fat diet, hearts from $\alpha$ MHC-caPKD1 mice develop an increased volume of the left-ventricular cavity and decreased thickness of the left-ventricular wall, characteristic of dilated cardiomyopathy. ${ }^{11}$ This cardiac remodeling observed in $\alpha$ MHC-caPKD1 mice on a low fat diet, had severe consequences because nearly all $\alpha \mathrm{MHC}-\mathrm{caPKD} 1$-mice died prematurely (within 20weeks of age), as might be expected based on a previous report ${ }^{11}$. The outward hypertrophic remodeling is associated with PKD1-mediated increase of glucose uptake. MEF2D, the main cardiac MEF2 isoform, ${ }^{17}$ appears to play a central role in this process. Upon activation, PKD1 is able to phosphorylate HDAC5, which results in nuclear export of HDAC5 and thus the activation of MEF2. ${ }^{18}$ Previously, it was shown that MEF2D is a transcription factor that activates genes that regulate growth and remodeling of the heart. ${ }^{12}$ Only recently, Ellwanger and coworkers proved that PKD activity is essential for MEF2-dependent skeletal muscle remodeling. ${ }^{19}$ Our results in MEF2D KO mice demonstrate that in addition to activation of a hypertrophic gene program, MEF2D also increases GLUT4 expression. Knocking-out MEF2D prevented PKD1 induced increase in GLUT4 expression. Apparently, MEF2D functions downstream of PKD1 in this pathway (Fig. 7). Previously, it has been shown that PKD1 KO mice display a diminished predisposition for (pathological) remodeling under stress. ${ }^{18}$ Together, these data demonstrate that PKD1 is able to link increased cardiac glucose uptake to hypertrophic remodeling of the heart, via activation of MEF2D. Our data are in line with other reports which showed that forced glucose uptake evokes dilated cardiomyopathy and deterioration of cardiac function in rodent models, such as rats supplemented with carnitine-palmitoyltransferase-1 inhibitors, ${ }^{20}$ and CD36-knockout mice. ${ }^{21,22}$ Also, it has been reported that increased glucose uptake coincides with hypertrophy of skeletal ${ }^{23}$ and cardiac muscle..$^{24,25}$ 


\section{Figure 7}

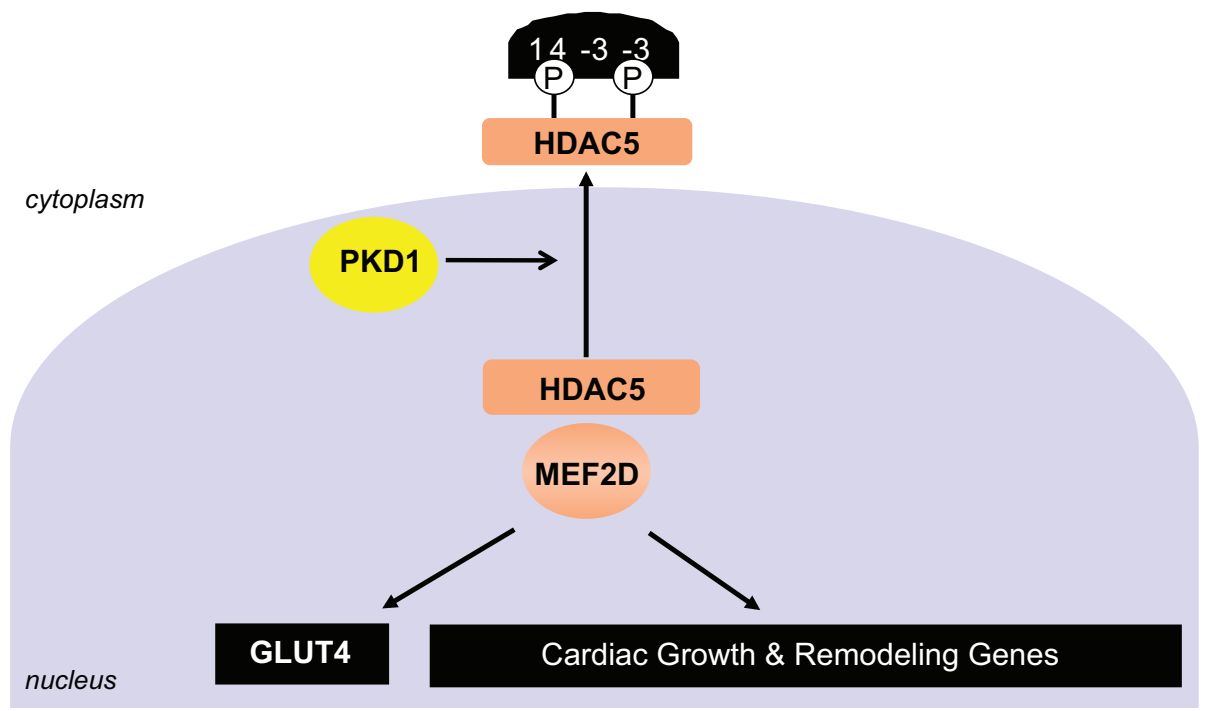

Figure 7. Schematic presentation of the PKD-HDAC5-MEF2 axis; a link between metabolism and hypertrophy

Upon activation, PKD phosphorylates HDAC5, which promotes its nuclear export and binding to 14-3-3. This results in the activation of MEF2D, which can activate as well as GLUT4 as other genes involved in hypertrophic remodeling. In this way, the PKD-HDAC5-MEF2D pathway links glucose uptake to hypertrophic remodeling of the heart.

In WT mice, exposure to high fat diet leads to cardiac insulin resistance ${ }^{26}$. In addition, high fat diet induced intra-myocardial TAG accumulation and an elevated basal cardiac LCFA uptake rate (which was insensitive to further insulin stimulation). These data are in agreement with earlier studies with high-fat feeding of rodents, which showed that the fold increase in intra-myocardial TAG content was related to the increase in elevated basal LCFA uptake. ${ }^{27}$ Hearts of WT mice on a high fat diet displayed additional characteristics of the lipid overloaded heart: decreased insulinstimulated Akt phosphorylation, decreased insulin-stimulated glucose uptake accompanied by increased left-ventricular wall thickness, decreased left-ventricular internal and external diameter. Our data are in line with reports in humans that type 2 diabetes is associated with left ventricular concentric hypertrophy, whereby the ejection fraction was preserved. ${ }^{28,}{ }^{29}$ Thus, $\alpha$ MHC-caPKD1 overexpression and high fat diet both induce hypertrophy, but different forms. However, the combination of PKD1 overexpression and a high fat diet eliminated the pathological effects of the separate interventions. Although $\alpha$ MHC-caPKD1 mice on a high fat diet showed elevated total body circulating insulin and glucose levels, due to whole body insulin resistance and similar to WT mice on a high fat diet, the hearts of $\mathrm{AMHC}$ caPKD1 mice did not display insulin resistance and associated hypertrophic remode- 
ling. In fact, the morphology and function of heart of $\alpha \mathrm{MHC}-\mathrm{caPKD} 1$ mice on a high fat diet were similar to those of WT mice on a low fat diet. Furthermore, $\mathrm{MMHC}$ caPKD1 mice on a high fat diet survived much longer than $\alpha$ MHC-caPKD1 mice on a low fat diet. This indicates that hearts of these mice on a high fat diet were rescued from glucose-induced dilated hypertrophy (observed in $\alpha$ MHC-caPKD1 mice on a low fat diet). Apparently, the high fat diet can prevent pathological remodeling in $\alpha$ MHC-caPKD1 mice by down-regulating PKD1. On the other hand, PKD1 overexpression can prevent cardiac remodeling and insulin resistance, induced by a high fat diet. Thus, the negative effects of a high fat diet and PKD1 overexpression balance each other. A report on $d b / d b$ mice, a rodent model for type-2 diabetes and cardiac lipid overload, describes a similar finding where overexpression of GLUT4 in the heart resulted in a balanced substrate utilization. ${ }^{30}$ Together, this supports the idea that proper functioning of the heart is associated with a balanced uptake and utilization of glucose and LCFA by cardiac muscle. ${ }^{22}$

\section{Concluding remarks}

We propose a model in which PKD1 is a key regulator of the substrate balance of the heart as its activation increases glucose uptake without affecting LCFA uptake. This occurs via MEF2D-mediated increase of GLUT4 expression. In the healthy heart, up-regulation of the PKD1-MEF2D pathway is unfavorable, as its activation leads to increased transcription of genes involved in both hypertrophic gene programming and glucose uptake, leading to dilated hypertrophic remodeling and eventually premature death. However, in a heart challenged by high plasma lipid levels, upregulation of this pathway offers protection against the development of concentric hypertrophic remodeling and insulin resistance by tipping the balance in substrate uptake towards glucose. This balanced cardiac substrate uptake is crucial for maintaining optimal cardiac function. Targeting the PKD1-MEF2D pathway offers the potential to control metabolic substrate switching in the failing heart.

\section{Acknowledgements}

We acknowledge the contribution of Felix Mottaghy, Emiel Beijer (Nuclear Medicine, Maastricht University), and of Agnieska Strzelecka (Pharmacology, Maastricht University). We thank Eric N. Olson (UT Southwestern Medical Center, Dallas, Texas) for providing the aMHC-caPKD1 and MEF2D KO mouse lines. This study was funded by the transnational University Limburg (tUL). 


\section{Refences}

1. Taegtmeyer H. Cardiac metabolism as a target for the treatment of heart failure. Circulation. 2004;110:894-896

2. Carley AN, Severson DL. Fatty acid metabolism is enhanced in type 2 diabetic hearts. Biochim Biophys Acta. 2005;1734:112-126

3. Glatz JF, Luiken JJ, Bonen A. Membrane fatty acid transporters as regulators of lipid metabolism: Implications for metabolic disease. Physiol Rev. 2010;90:367-417

4. Zisman A, Peroni OD, Abel ED, Michael MD, Mauvais-Jarvis F, Lowell BB, Wojtaszewski JF, Hirshman MF, Virkamaki A, Goodyear LJ, Kahn CR, Kahn BB. Targeted disruption of the glucose transporter 4 selectively in muscle causes insulin resistance and glucose intolerance. Nat Med. 2000;6:924-928

5. Kramer HF, Witczak CA, Fujii N, Jessen N, Taylor EB, Arnolds DE, Sakamoto K, Hirshman MF, Goodyear LJ. Distinct signals regulate as160 phosphorylation in response to insulin, aicar, and contraction in mouse skeletal muscle. Diabetes. 2006;55:2067-2076

6. Bonen A, Holloway GP, Tandon NN, Han XX, McFarlan J, Glatz JF, Luiken JJ. Cardiac and skeletal muscle fatty acid transport and transporters and triacylglycerol and fatty acid oxidation in lean and zucker diabetic fatty rats. Am J Physiol Regul Integr Comp Physiol. 2009;297:R12021212

7. Erion DM, Shulman GI. Diacylglycerol-mediated insulin resistance. Nat Med. 2010;16:400-402

8. Boudina S, Abel ED. Diabetic cardiomyopathy, causes and effects. Rev Endocr Metab Disord. 2010;11:31-39

9. Loganathan R, Bilgen M, Al-Hafez B, Zhero SV, Alenezy MD, Smirnova IV. Exercise training improves cardiac performance in diabetes: In vivo demonstration with quantitative cine-mri analyses. J Appl Physiol. 2007;102:665-672

10. Luiken JJ, Vertommen D, Coort SL, Habets DD, El Hasnaoui M, Pelsers MM, Viollet B, Bonen A, Hue L, Rider MH, Glatz JF. Identification of protein kinase $d$ as a novel contraction-activated kinase linked to GLUT4-mediated glucose uptake, independent of ampk. Cell Signal. 2008;20:543-556

11. Harrison BC, Kim MS, van Rooij E, Plato CF, Papst PJ, Vega RB, McAnally JA, Richardson JA, Bassel-Duby R, Olson EN, McKinsey TA. Regulation of cardiac stress signaling by protein kinase d1. Mol Cell Biol. 2006;26:3875-3888

12. Olson EN, Backs J, McKinsey TA. Control of cardiac hypertrophy and heart failure by histone acetylation/deacetylation. Novartis Found Symp. 2006;274:3-12; discussion 13-19, 152-155, 272-156

13. Harrison BC, Kim MS, van Rooij E, Plato CF, Papst PJ, Vega RB, McAnally JA, Richardson JA, Bassel-Duby R, Olson EN, McKinsey TA. Regulation of cardiac stress signaling by protein kinase d1. Molecular and cellular biology. 2006;26:3875-3888

14. Kim Y, Phan D, van Rooij E, Wang DZ, McAnally J, Qi X, Richardson JA, Hill JA, Bassel-Duby R, Olson EN. The mef2d transcription factor mediates stress-dependent cardiac remodeling in mice. J Clin Invest. 2008;118:124-132

15. Habets DD, Coumans WA, Voshol PJ, den Boer MA, Febbraio M, Bonen A, Glatz JF, Luiken JJ. Ampk-mediated increase in myocardial long-chain fatty acid uptake critically depends on sarcolemmal CD36. Biochem Biophys Res Commun. 2007;355:204-210

16. Folch J, Lees M, Sloane Stanley GH. A simple method for the isolation and purification of total lipides from animal tissues. J Biol Chem. 1957;226:497-509

17. Kelber J, Slatopolsky E, Delmez JA. Acute effects of different concentrations of dialysate magnesium during high-efficiency dialysis. Am J Kidney Dis. 1994;24:453-460

18. Fielitz J, Kim MS, Shelton JM, Qi X, Hill JA, Richardson JA, Bassel-Duby R, Olson EN. Requirement of protein kinase D1 for pathological cardiac remodeling. Proc Natl Acad Sci U S A. 2008;105:3059-3063

19. Ellwanger K, Kienzle C, Lutz S, Jin ZG, Wiekowski MT, Pfizenmaier K, Hausser A. Protein kinase D controls voluntary-running-induced skeletal muscle remodelling. Biochem J. 2011;440:327324 
20. Hulsmann WC, Peschechera A, Schneijdenberg CT, Verkleij AJ. Comparison of the effects of carnitine palmitoyltransferase- 1 and -2 inhibitors on rat heart hypertrophy. Cardioscience. 1994;5:193-197

21. Febbraio M, Abumrad NA, Hajjar DP, Sharma K, Cheng W, Pearce SF, Silverstein RL. A null mutation in murine cd36 reveals an important role in fatty acid and lipoprotein metabolism. $J$ Biol Chem. 1999;274:19055-19062

22. Glatz JF, Bonen A, Ouwens DM, Luiken JJ. Regulation of sarcolemmal transport of substrates in the healthy and diseased heart. Cardiovasc Drugs Ther. 2006;20:471-476

23. Young JC, Kandarian SC, Kurowski TG. Skeletal muscle glucose uptake following overloadinduced hypertrophy. Life Sci. 1992;50:1319-1325

24. Abel ED, Kaulbach HC, Tian R, Hopkins JC, Duffy J, Doetschman T, Minnemann T, Boers ME, Hadro E, Oberste-Berghaus C, Quist W, Lowell BB, Ingwall JS, Kahn BB. Cardiac hypertrophy with preserved contractile function after selective deletion of GLUT4 from the heart. J Clin Invest. 1999;104:1703-1714

25. Kato T, Niizuma S, Inuzuka Y, Kawashima T, Okuda J, Tamaki Y, Iwanaga Y, Narazaki M, Matsuda T, Soga T, Kita T, Kimura T, Shioi T. Analysis of metabolic remodeling in compensated left ventricular hypertrophy and heart failure. Circ Heart Fail.3:420-430

26. Wright JJ, Kim J, Buchanan J, Boudina S, Sena S, Bakirtzi K, Ilkun O, Theobald HA, Cooksey RC, Kandror KV, Abel ED. Mechanisms for increased myocardial fatty acid utilization following short-term high-fat feeding. Cardiovasc Res. 2009;82:351-360

27. Ouwens DM, Diamant M, Fodor M, Habets DD, Pelsers MM, El Hasnaoui M, Dang ZC, van den Brom CE, Vlasblom R, Rietdijk A, Boer C, Coort SL, Glatz JF, Luiken JJ. Cardiac contractile dysfunction in insulin-resistant rats fed a high-fat diet is associated with elevated CD36-mediated fatty acid uptake and esterification. Diabetologia. 2007;50:1938-1948

28. Eguchi K, Kario K, Hoshide S, Ishikawa J, Morinari M, Shimada K. Type 2 diabetes is associated with left ventricular concentric remodeling in hypertensive patients. Am J Hypertens. 2005;18:23-29

29. Maciver DH, Townsend M. A novel mechanism of heart failure with normal ejection fraction. Heart. 2008;94:446-449

30. Belke DD, Larsen TS, Gibbs EM, Severson DL. Altered metabolism causes cardiac dysfunction in perfused hearts from diabetic (db/db) mice. Am J Physiol Endocrinol Metab. 2000;279:E11041113 
CHAPTER 8

\section{Discussion}


A number of diseases, such as diabetes and hypertension, induce changes in cardiac contraction and metabolism and eventually result in hypertrophic remodeling. This thesis focuses on the role of PKD in pathways that direct these processes. PKD is implicated in conveying extra-cellular signals to both the contractile apparatus and the nucleus of the cardiomyocyte. Also, PKD plays a role when prolonged stimulation of these signaling cascades has to be translated in structural adaptations to cope with changed metabolic and contractile demands. Over the last decade a number of pathways have been, more or less, mapped, but our knowledge is limited, in particular, concerning the crosstalk of the different pathways. Kinases are essential players in these pathways as they may integrate distinct signaling cascades. As such, PKD provides a good example of a regulator at the crossroad of different pathways.

\section{Signaling pathways in contraction}

Of the receptors that function as the cardiomyocyte windows to the outside world, the adrenergic receptors (ARs) appear most important. The heart has $\alpha$ and $\beta$ adrenergic receptors which can be stimulated by different catecholamines. ${ }^{1}$ Signaling cascades started by activation of the ARs can be aimed at contractility, metabolism or cell growth. The $\beta$-ARs are most abundant in the heart and can be subdivided in 2 types; the $\beta 1$-ARs and $\beta 2$-ARs. ${ }^{2}$ Physical activities, such as exercise, will predominantly stimulate $\beta 1-A R s$ by adrenalin and noradrenalin released in the blood. This will lead to an increased cardiac output by a raise of the heart rate (positive chronotropic effect), an increased impulse conduction, and an stronger contraction. ${ }^{3}$ At the cellular level, downstream of $\beta 1$-ARs, signaling cascades will become activated to support contraction, for instance by facilitating the necessary increase in uptake of high-energy substrates. One of the first membrane bound enzymes that will be activated via $\beta 1-A R$ is adenylate cyclase. This enzyme will convert ATP into cAMP. The generated CAMP will bind to protein kinase A (PKA), which will subsequently activate L-type $\mathrm{Ca}^{2+}$ channels [Fig. 1A]. ${ }^{4}$ This will raise intracellular $\mathrm{Ca}^{2+}$ concentrations and promote binding of $\mathrm{Ca}^{2+}$ to troponin which starts contraction. Also, PKA will phosphorylate sarcomeric proteins such as Tnl, cMyBP-C. Phosphorylation of these proteins is associated with decreased $\mathrm{Ca}^{2+}$ sensitivity and increased rates of crossbridge cycling. ${ }^{5}$ This will enhance force generation and relaxation of the cardiomyocytes, resulting in a more powerful contraction. In cMyBP-C KO mice, left ventricular functional capacity was significantly diminished and the increase in myocardial contractility, following $\beta$-adrenergic stimulation, was markedly attenuated. ${ }^{6}$ This suggests that the absence of $\mathrm{CMyBP-C}$ attenuates the inotropic reserve in left ventricular contractility, typically observed as a consequence of $\beta$-adrenergic stimulation. Importantly, $\beta 1-A R$ stimulation also activates a second pathway; phospholipase $C$ 
(PLC) will be activated which leads to the generation of DAG and intracellular messengers inositol( $1,4,5)$ tri-phosphate $(\mathrm{PI} 3) .^{7}$ Production of diacetylglycerol (DAG) will activate another set of kinases, the PKCs [Fig. 1]. ${ }^{7}$ Some of these PKC isoforms are able to subsequently phosphorylate PKD. ${ }^{8,9}$ As we and others showed, PKD too can phosphorylate $\mathrm{Tnl}$ and $\mathrm{CMyBP}-\mathrm{C}$ and enhance cardiomyocyte contraction (Chapter 5). ${ }^{10}$ Although PKA, PKC and PKD all strengthen contraction, subtle differences in the way these kinases control contraction, have been described. ${ }^{9,11,12}$ For, each kinase phosphorylates different residues of the target molecules, which introduces specific spatial conformations. More recently it has become clear that a strict regulation of the cytoplasmic location and time of action contributes substantially to the distinct role of each of these kinases. ${ }^{13}$

Fig 1

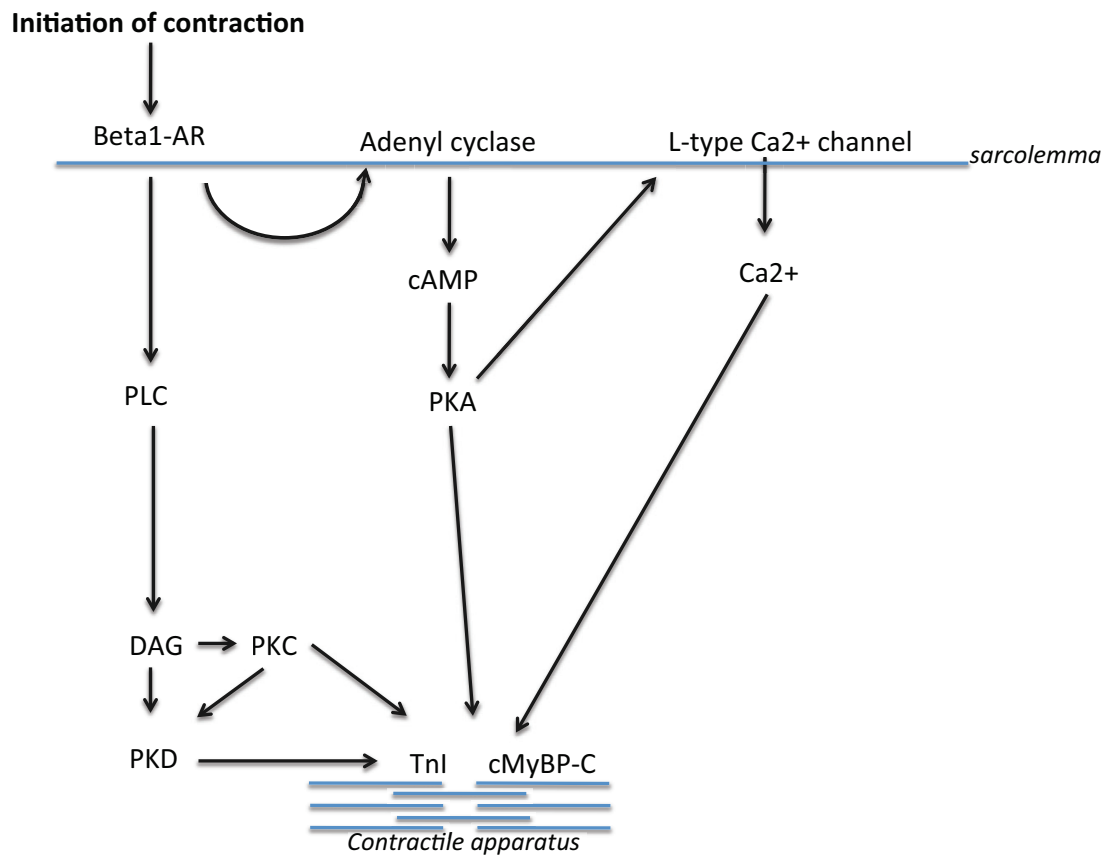

Figure 1: $\beta 1$-ARs signaling cascades activated during contraction. Stimulation of $\beta 1$-AR will lead to the activation of at least two pathways. One of the first membrane bound enzymes that will be activated via $\beta 1-A R$ is adenylate cyclase. This enzyme will convert ATP into cAMP. The generated cAMP will bind PKA, which will subsequently activate L-type $\mathrm{Ca} 2+$ channels, which promotes binding of $\mathrm{Ca}^{2+}$ to troponin. Also, PKA will phosphorylate sarcomeric proteins such as Tnl, CMyBP-C to strengthen contraction. The second enzyme which will be stimulated via $\beta 1-A R$ is PLC. PLC activation will lead to the generation of DAG, which activates PKC. Some PKC isoforms are able to subsequently phosphorylate PKD. Also PKC and PKD are able to phosphorylate $\mathrm{Tnl}$ and $\mathrm{CMYBP}-\mathrm{C}$ to strengthen contraction. $61-A R: \beta 1$ adrenergic receptor, $P K A$ : protein kinase A, PKC: protein kinase C, PKD: protein kinase $D$, Tnl: troponin I, $C M y B P-C$ : cardiac myosin binding protein-C, $P L C$ : phopsholipase $C, D A G$ : diacylglycerol 
As mentioned before, $\beta$-AR stimulation of adenyl cyclase generates cAMP. This small molecule would rapidly spread over the entire cytoplasm if no restrictions would be operative. It then would activate many cellular processes, which may not all fit in with a well-organized onset of contraction. To make the situation even more complicated, other receptors transduce their signal also via CAMP, but evoke functionally different effects. A temporal and spatial order appears to be mandatory to keep this CAMP second messenger system operational. Over the last decade the outlines of such an organization, comprising kinases and proteins that can function as "docking stations" and "insulators", has emerged. Although this thesis deals largely with kinases, the docking proteins and insulators may have an as important function as the kinases. One class of the docking proteins is A-kinase anchoring proteins (AKAPs) [Fig. 2]. ${ }^{13}$ As the word says, AKAPs can bind PKA. However, it has become evident that AKAPs serve a more general role. They are involved in numerous processes that regulate synchronization and spatial restriction of signaling processes on organelles and membranes. ${ }^{13}$ To perform this function, AKAPs can bind kinases, phosphatases and other signaling molecules [Fig. 2]. For instance, AKAP18 $\alpha$ (also called AKAP15 $\alpha$ ) directly affects L-type $\mathrm{Ca}^{2+}$ channels in cardiomyocytes, and is instrumental to $\mathrm{Ca}^{2+}$ entry into the cytosol, thereby facilitating $\beta$-ARmediated increase in cardiomyocyte contractility. ${ }^{14}$ On the other hand AKAPs can integrate cellular signaling. For example, in cardiomyocytes, AKAP-lbc functions as a scaffolding protein for PKA and PKC to mediate activation of a third kinase, PKD. ${ }^{15}$ The activation of PKC or PKD depends on second messengers such as DAG, whereas PKA activation relies on CAMP. These kinases can interact after binding to AKAPs and as such these AKAPs will functions as a point of convergence of the CAMP and phospholipid signaling pathways. Moreover, AKAPs can direct such anchored enzyme pools to their physiological substrates or to specific subcellular locations. ${ }^{16}$ For instance, AKAPs tether PKA to particular cellular compartments and thereby limit PKA's access to a subset of its substrates. Although AKAPs provide a base to restrict CAMP-dependent PKA signaling to well-defined cellular sites, the tethering of PKA by AKAP is, by itself, not sufficient to compartmentalize and control CAMPdependent pathways, since CAMP may diffuse throughout the whole cell. Complementary, phosphodiesterases (PDEs) establish gradients of CAMP by local hydrolysis of this second messenger and thereby limit PKA activity in space and time. ${ }^{17}$ Thus, degradation of CAMP by PDEs confines CAMP to limited domains in the cytoplasm and prevents it from reaching its target proteins, such as PKA and so-called Epacs (exchange proteins directly activated by CAMP) [Fig. 2]. ${ }^{18}$ 
Fig 2

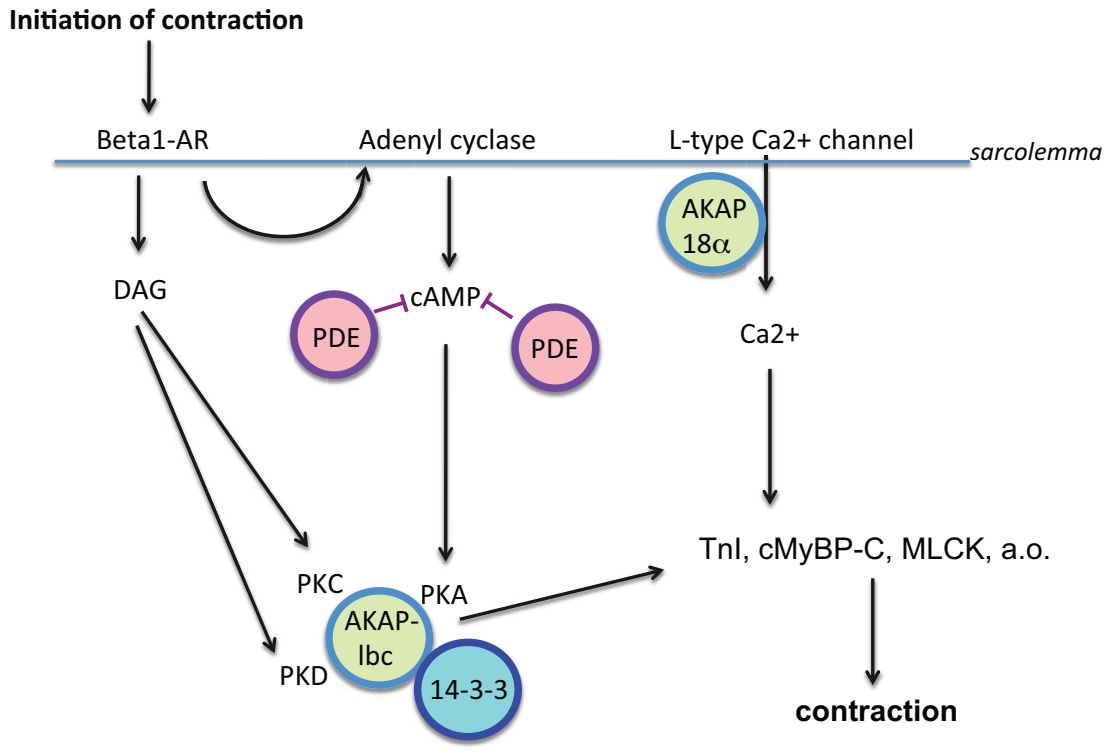

Figure 2: Spatial restriction of signaling cascades downstream of $\beta 1$-ARs. AKAPs can bind kinases, phosphatases and other signaling molecules by tethering these molecules to particular cellular compartments, and in this way guide signaling to target. Downstream of $\beta 1$-ARs AKAP-lbc functions as a scaffolding protein for PKA and PKC to mediate activation of PKD. Upon binding of PKA to AKAP, PKD will be released into the cytoplasm and can phosphorylate several substrates such as $\mathrm{Tnl}$ and cMy7BP-C. AKAP18 $\alpha$ directly affects L-type $\mathrm{Ca}^{2+}$ channels in cardiomyocytes to increase intracellular $\mathrm{Ca}^{2+}$, thereby facilitating $\beta$-AR-mediated increase in cardiomyocyte contractility. PDEs are able to limit PKA activity in space and time by local hydrolysis of cAMP. This way these molecules create corridors leading and integrating signals to their destination. B1-AR: $\beta 1$ adrenergic receptor, AKAP: A-kinase anchoring protein, $P K A$ : protein kinase $\mathrm{A}, P K C$ : protein kinase $\mathrm{C}, P K D$ : protein kinase $\mathrm{D}, T h \mathrm{l}$ : troponin I, $C M y B P-C$ : cardiac myosin binding protein-C, $P D E$ :Phosphodiesterases

In line with this, Zaccolo and Pozzan (2002) demonstrated elegantly that increases of cAMP concentrations in cardiomyocytes occur in discrete micro-domains near Ttubules and Z-discs. ${ }^{19}$ To generate cAMP gradients, PDEs associate with a variety of proteins. PDE3, the predominant cardiac isoform, interacts mainly with the 14-3-3 protein. ${ }^{20}$ In addition, PDE have been reported to bind to AKAPs and may play a role at that level of control. ${ }^{21}$ Surprisingly, in PDE3A-knockout mice no change in contraction and relaxation of cardiomyocytes has been observed, indicating a redundancy in this class of proteins. ${ }^{22}$ Taking into account the number of PDEs isozymes, their relative abundance and redundancy, it will take time to define their precise role in cardiomyocytes. Thus, contraction signaling comprises a number of signaling cascades, which are carefully controlled in space and time. Signals are transferred 
between stations, provided by proteins such as AKAPs, traveling through corridors limited by catabolic proteins such as PDEs.

The role of PKD in metabolic pathways

As cardiac cells lack significant stores of energy substrates, increased contraction will quickly utilize all ATP available and cause a rise in both AMP/ATP ratio and mitochondrial ROS production. ${ }^{23,24}$ The increase in AMP and ROS production will, in the wake of the contraction signaling, stimulate a second wave of signaling cascades, which will raise uptake of the high-energy substrates glucose and long chain fatty acids (LCFA) by recruitment of membrane transporters GLUT4 and CD36 to the sarcolemma [Fig. 3]. ${ }^{25}$ Increased AMP and ROS production will activate at least two pathways. Binding of AMP, in combination with phosphorylation of Thr172 by LKB1, will activate AMPK. ${ }^{26}$ The generation of ROS, as a consequence of mitochondrial oxidation, will lead to the binding of DAPK to PKD, which activates the latter one [Chapter 3]. Both AMP-AMPK and DAPK-PKD pathways will stimulate a translocation of GLUT4 to the plasma membrane and will lead to an increased glucose uptake [Chapter 3]. AMP-induced AMPK also stimulates CD36-mediated LCFA uptake. The demonstrated selectivity of PKD for glucose uptake is interesting in relation to diabetes [Chapter 6, 7]. Since AMPK as well as PKD advance glucose uptake, one would expect some crosstalk between these two kinases. This was tested in both AMPK KO and PKD KO mice [Chapter 3]. However, so far no proof for such crosstalk has been found. The mechanism by which PKD (and AMPK) induces GLUT4 translocation remains elusive. There is a lot of circumstantial evidence tying GLUT4 vesicles to actin and to various PI-kinases, but the overall picture is confusingly incomplete. $^{27}$ It has been shown that phosphatidylinositol-4-kinase-III $\beta$ (PI4KIII $\beta$ ), which is present at the trans-Golgi-network (TGN), is a physiological substrate of PKD that regulates vesicular transport to the plasma membrane. ${ }^{28}$ Furthermore, PI4KIII $\beta$ has been shown to be present in GLUT4 vesicles. ${ }^{29}$ Therefore, PI4KIII $\beta$ may function as an interface between vesicles and the cytoskeleton, more in particular actin fibers of the microfilaments. 
Fig 3

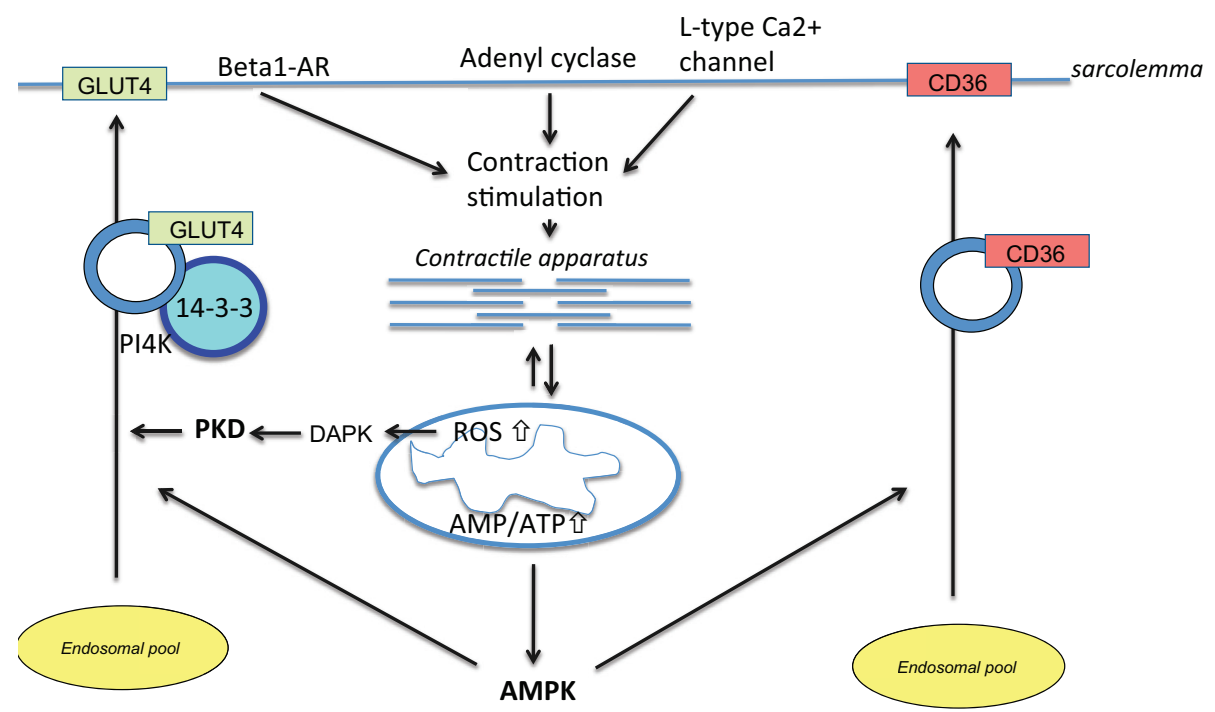

Figure 3: Activation of metabolic pathways upon contraction through B1-AR stimulation. Increased contraction will quickly utilize all ATP available and cause a rise in AMP/ATP ratio and mitochondrial ROS production. Binding of AMP will activate AMPK. The generation of ROS will lead to the binding of DAPK to PKD, which will activate the latter one. Both AMP-AMPK and DAPK-PKD pathways will stimulate translocation of GLUT4 from endosomal pools to the plasma membrane and will lead to an increased glucose uptake. For PKD a possible mechanism is the phosphorylation of PI4K, which may give way to PI4K binding to 14-3-3 and mediate GLUT4 transport to the plasma membrane. AMP-induced AMPK also stimulates translocation of CD36 from endosomal pools to the plasma membrane to increase LCFA uptake. 61$A R: \beta 1$ adrenergic receptor, $A M P K$ : AMP-activated protein kinase, ROS: reactive oxygen species, DAPK: death associated protein kinase, PKD: protein kinase D, GLUT4: glucose transporter 4, PI4K: phosphatidylinositol 4 kinase

Using PI4KIII $\beta$ siRNA we obtained preliminary results, that showed that knock-down of PI4KIII $\beta$ prevented contraction-induced GLUT4 translocation, but had no effect on contraction-induced CD36 translocation in HL1 cells [Fig. 4]. 
Fig 4
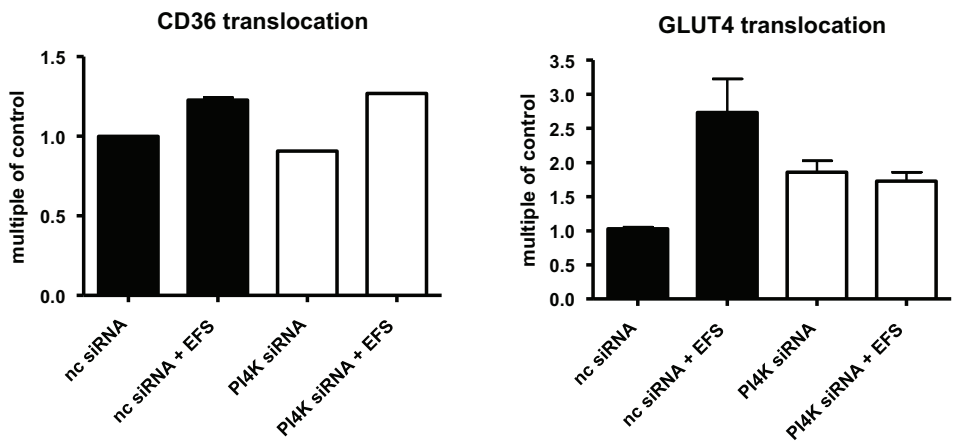

Figure 4: Effect of the use of PI4KIII $\beta$ siRNA on GLUT4 and CD36 translocation in contracting HL1 cells. PI4KIII $\beta$ siRNA inhibits contraction-stimulated GLUT4 translocation to the sarcolema, but not contractionstimulated CD36 translocation. NC siRNA = non-coding siRNA, $2 \mathrm{~Hz}=$ electric field stimulation = contraction

This indicates that phosphorylation of PI4K by PKD is another step in the GLUT4 translocation pathway. Such phosphorylation may give way to PI4K binding to 14-33, a well-known "sweeper" of phosphorylated proteins [Fig. 3]. ${ }^{30}$ One may hypothesize that 14-3-3 enables GLUT4/endosome transport via the cytoskeleton, since experiments in yeast have shown that 14-3-3 interacts with proteins, such as kinesin light chain-2 and dynein that are involved in diverse aspects of membrane or Golgi trafficking. ${ }^{31-33}$ These data, therefore, suggest that PI4K is a downstream substrate of PKD, and that after interaction with 14-3-3 it may facilitate GLUT4 transport to the plasma membrane by providing a carrier that has access to the highways of the cytoskeleton.

In the context of cardiomyocyte signaling, 14-3-3 deserves more attention, although it has no enzymatic activity of its own. However, it can regulate cellular processes by binding proteins. ${ }^{34}$ Structural studies in combination with sequence analyses have shown that 14-3-3 proteins have a preference for phosphorylated substrates, which they bind with their anti-parallel bivalent binding sites. ${ }^{35}$ In addition to the masking of active sites and imposing conformational changes in proteins, 14-3-3 proteins are also capable of nucleo-cytoplasmic shuttling of proteins. During nutrient deprivation in yeast, PI4K relocates from the TGN to the cytoplasm and into the nucleus after binding to 14-3-3. This relocation is rapidly reversed upon restoration of nutrient supply. However, the purpose of this nucleo-cytoplasmic shuttling of PI4K is not known. In higher eukaryotes 14-3-3 proteins have also been shown to be involved in nucleo-cytoplasmic shuttling of HDAC5. ${ }^{36,37}$ Although PKD, AMPK, and PKA can 
phosphorylate HDAC5, only PKD- and AMPK-induced HDAC5 phosphorylation promotes binding to 14-3-3 and subsequent export of HDAC5 out of the nucleus. ${ }^{38,39}$ On the contrary, phosphorylation of HDAC5 by PKA prevents this process. ${ }^{40}$ The involvement of 14-3-3 proteins in nucleo-cytoplasmic shuttling appears to position them to structures around the nucleus such as the Golgi apparatus. ${ }^{30}$ Thus, 14-3-3 appears to be able to guide and control signaling in the cell.

\section{Signaling pathways in cardiac hypertrophy}

The heart has a limited repertoire to compensate for changed or unfavorable conditions, such as pregnancy, ${ }^{41}$ exercise, genetic mutations, and diabetes. ${ }^{42}$ Often hypertrophy is the response to increased workload or insults. So, upon more intense stimulation, cardiomyocytes will at first respond with stronger contraction and larger import of metabolic substrates. However, when an increase in workload continues, the cardiomyocyte has to translate the increased demand into structural changes that allow it to cope with the new situation. If the increased workload leads to a hypertrophic response with a steady state, as in pregnancy and exercise, it is considered to be physiological. If it leads to progressive contractile dysfunction, as in the diabetes, it is considered to be pathological.

A sustained high workload will lead to changes in adrenergic receptor responses, as a continuously higher concentration of catecholamines will induce a downregulation of $\beta 1 A R$ on the cell membrane in favor of $\beta 2 A R$. The diminished role of $\beta 1 A R$ can also be result of a negative feedback-loop involving PKA, $\beta A R K 1$ or PKC, which all are able to phosphorylate the receptor, thereby changing its structure, and making it less sensitive to catecholamines. How these changes in receptor profile are translated into signaling cascades involved in regulating cardiac hypertrophy is still a matter of debate. Most likely, there is no such pathway as a "hypertrophy pathway". There is evidence that cardiac hypertrophy can be triggered through activa-

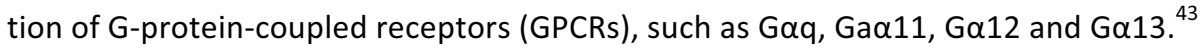
Ligands for these receptors include norepinephrin, angiotensin, endothelin, trombin and LPA. ${ }^{44,45}$ These GPCRs activate RhoA via guanine nucleotide exchange factors (RhoGEFs). Transfer of GTP activates RhoA, which then starts a signaling cascade via Rho-associated coiled-coil containing protein kinase (ROCK), lead to hypertrophy and fibrosis. ${ }^{46}$ Inhibition of RhoGEF activity can be achieved by PKA phosphorylation of AKAP-lbc and subsequent 14-3-3 protein binding to the phosphorylation site of AKAP. This will prevent hypertrophy. ${ }^{13,47}$ In contrast, $\alpha$-AR activation by phenylephrine increases the RhoGEF activity in cardiomyocytes by elevating AKAP expression, and leads to the development of cardiac hypertrophy. In yet another pathway, activation of PI3K by $\beta A R K 1$, results in Akt-mediated FOXO3a inhibition, 
which also promotes hypertrophy. ${ }^{48}$ In addition to this incomplete compilation of pathways that lead to cardiac hypertrophy, several kinases, amongst which PKC, CaMK and PKD have been described to induce hypertrophy. It is beyond the scope of this thesis to give a complete overview of the multitude of factors that are involved in the development and prevention of cardiac hypertrophy. ${ }^{49}$ Here we focus on pathways with a direct role for PKD, and their interaction with other signaling cascades [Fig. 5] (as discussed below).

The role of PKD in hypertrophic signaling

In cardiomyocytes the role of PKD has been recognized in a growing number of processes. From this thesis [Chapter 3,7] and other publications it is clear that PKD is involved in cardiac glucose metabolism. Also, this thesis illuminates its role in contraction regulation via CMyBP-C [Chapter 5] and troponin-I. ${ }^{50}$ Further, several studies have demonstrated the role of PKD in cell survival. ${ }^{51,52}$ Finally, PKD directly affects the development of cardiac hypertrophy by phosphorylation of HDAC5 [Chapter 7]. ${ }^{49,53}$ HDAC5 KO mice display hypertrophy compared to wild-type littermates. HDAC5 appears to play a role in the suppression of a subset of cardiac stress signals and also in the control of cardiac development. ${ }^{54}$ One of the mechanisms by which HDAC5 suppresses cardiac stress is by binding to MEF2. PKD-mediated HDAC5 phosphorylation leads to an uncoupling of MEF2 and HDAC5, and subsequent shuttling of HDAC5 out of the nucleus, and activation of MEF2 and calmodulin binding transcription activators (CAMTA), which both lead to induction of growth and remodeling genes. ${ }^{55}$ These different roles may run along separate pathways and started via distinct receptors.

Norepinephrine (NE), via the $\alpha 1-A R$, and angiotensin II (AngII), via the $\beta A R s$, are strong activators of cardiac PKD. In addition, PKD can be activated in response to hypertension, pressure overload, and chronic neurohormonal signaling (15-19). A prolonged NE or Angll stimulation leads to hypertrophy (and apoptosis). Angllinduced PKD1 activation increases expression of atrial natriuretic factor (ANF), brain natriuretic peptide (BNP) and $\beta$-myosin heavy chain ( $\beta-\mathrm{MHC})$, all of which are indicative for hypertrophy. More evidence for involvement of PKD in Angll- or pressure overload-induced hypertrophy was obtained from PKD knockout mice. Mice with cardiac-specific deletion of PKD1 were viable and showed diminished hypertrophy, fibrosis, and fetal gene activation as well as improved cardiac function in response to pressure overload or chronic adrenergic and Angll signaling. ${ }^{56}$ However, pathways employing other signaling proteins may be needed to develop ventricular hypertrophy. Integration of the different pathways appears paramount and may rely on the presence of the above discussed molecules, such as APAKs, PDE and 143-3. As discussed above, these proteins interact with a number of protein kinases, and may integrate signaling cascades coming from different receptors. 
Results obtained from experiments with caPKD1 transgenic mice ${ }^{57}$ [Chapter 7] connect GLUT4 synthesis/translocation with hypertrophy. It has been shown that activation of $\mathrm{PKD}^{57}$, as well as $\mathrm{CaMK}^{58}$, induces pathological cardiac remodeling by phosphorylating class II HDACs and relieving MEF2 repression. Both PKD and CaMK phosphorylate Ser259 of HDAC5. Thus, PKD promotes export of HDAC5 out of the nucleus, which activates MEF2 and leads to both GLUT4 gene expression and activation of fetal gene expression. Interestingly, also AKAP-Ibc seems to be involved in this pathway. AKAP-Lcb has been found to be upregulated in response to hypertrophic stimuli. It enhances the efficiency of signaling through the PKD-HDAC5-MEF2 pathway that elicits the fetal gene response. ${ }^{38}$ Knockdown of AKAP-Lbc prevents the development of hypertrophy. ${ }^{59}$ A study by Mayers et al showed that loss of function of AKAP-Lcb (also known as AKAP13) in the mouse resulted in a thin myocardium and an embryonic lethal phenotype. ${ }^{60}$ Furthermore, AKAP-Lcb is required for cardiac development and induction of normal levels of MEF2C during cardiomyocyte differentiation. $^{60}$ On the contrary, PKA has anti-hypertrophic effects by preventing HDAC5 nuclear export through phosphorylating HDAC5-Ser $280 .^{40}$ Recently it has been shown that the association profile of PKA with several AKAPs is severely altered in the (human) failing heart. ${ }^{61}$ These changes seem to be part of a framework that altogether accommodate reduced contractility, rhytm and abberant $\mathrm{Ca}^{2+}$ handling of the failing heart. ${ }^{61}$ Thus, PKA activation prevents hypertrophic remodeling under normal conditions. However in case of heart failure, the AKAP-PKA signaling is deregulated and PKA is not able to prevent cardiac remodeling anymore. The mechanism for PKD/PKA/AKAP cooperation has not been elucidated yet. A possible explanation might be that during "hypertrophic" conditions, only PKA binds AKAPIbc, while 14-3-3 does not. This will lead to activation of RhoGEF and apparently also to PKD-mediated HDAC5 phosphorylation. This phosphorylated HDAC5 then binds to 14-3-3 and shuttles out of the nucleus.

Interestingly, in skeletal muscle, AMPK is also able to phosphorylate HDAC5Ser259. ${ }^{62}$ This AMPK-mediated HDAC5 phosphorylation leads to the activation of GLUT4 gene expression. ${ }^{39,63}$ Recently, it was shown that exercise training increases the nuclear MEF2A content and binding of MEF2A to the GLUT4 gene by an AMPK $\alpha 2$-dependent mechanism. ${ }^{63}$ However, intracellular signaling molecules other than AMPK $\alpha 2$ seem to be involved in regulating training-induced HDAC5 nuclear export. Furthermore, although AMPKa2 mediates training-induced increase in binding of MEF2A to the GLUT4 gene, AMPK 22 has not been shown to have an essential role in regulating training-induced GLUT4 expression in skeletal muscle. ${ }^{63}$ Thus, also PKD might be involved in the activation of exercise-induced MEF2-GLUT4 axis. Whether AMPK also stimulates cardiac hypertrophy via the HDAC5-MEF2 pathway is not known. In my opinion this could be possible since both $\mathrm{AMPK}^{64}$ and MEF2 expression are increased during exercise-induced hypertrophy. In contrast, the PKD- 
HDAC5-MEF2 pathway has been demonstrated to stimulate pathological cardiac hypertrophy and results in a decreased lifespan (Chapter 7). Thus, it appears that MEF2 is activated during both physiological and pathological hypertrophy. Although, nothing is really known about the role of PKD in physiological hypertrophy, there are some data that link PKD to exercise training. PKD becomes phosphorylated and thus activated during increased contraction frequency. Also, both exercise training ${ }^{65}$ and PKD overexpression reduce cardiac insulin resistance, and increase total GLUT4 levels. ${ }^{66}$ Furthermore, it has been shown that during exercise, GLUT4 gene expression is increased by activation of MEF2A. ${ }^{67}$ The upstream events to induce MEF2 activation during exercise are not fully understood, but PKD and AMPK might play an important role in this process. ${ }^{67,68}$ Thus, it is likely that during physiological and pathological hypertrophy, similar but not the same pathways become activated. Comparison of the development of physiological and pathological hypertrophy in time and may provide insight in the molecular mechanisms that turn hypertrophy pathological.

Fig 5

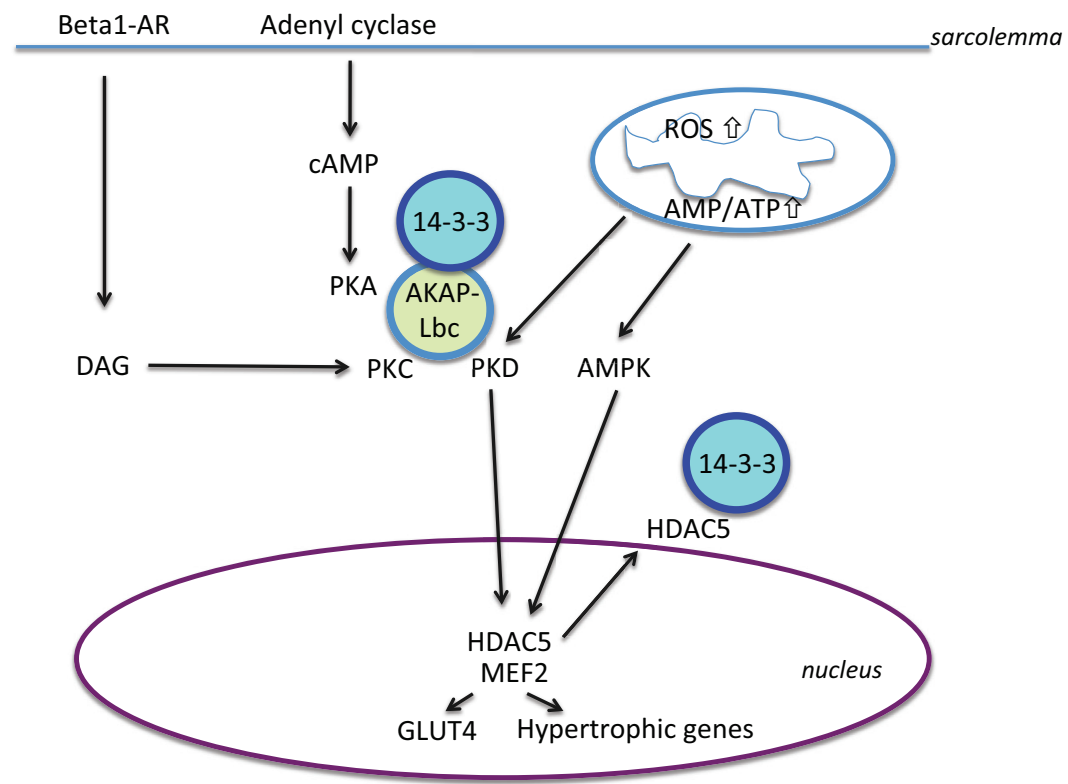

Figure 5 Continuous B-AR stimulation induces hypertrophic remodeling. Stimulation of $\beta A R$, to increase contraction, activates PKC via DAG, and PKA via adenyl cyclase. This leads to an increase of mitochondrial oxidation with subsequent increased production of ROS and AMP/ATP ratio, activating PKD and AMPK respectively. AKAP-Lcb is up-regulated in response to $\beta A R$ stimulation and supposed to integrated the different pathways that affect contraction and metabolic induced hypertrophy. This way the efficiency of signaling through the PKD-HDAC5-MEF2 pathway can be enhanced. Continuous PKD activation leads to the phosphorylation of HDAC5, which induces binding of HDAC5 to 14-3-3 and nuclear export of HDAC5. 
Subsequently, MEF2 will be activated, which leads to and increase in GLUT4 gene expression and genes involved in hypertrophic remodeling. Also, AMPK activation may lead to phosphorylation of HDAC5 with effects similar to those evoked by PKD. The role of AMPK in increasing MEF2-mediated hypertrophic gene expression is not known yet.

PKD: protein kinase D, HDAC5: histone deacetylase 5, MEF2: myocytes enhancer factor 2, AKAP-Ibc: Akinase anchoring protein-lbc, AMPK: AMP-activated protein kinase, GLUT4: glucose transporter 4

\section{The impact of high fat diet on metabolic and hypertrophic signaling}

Dietary lipids are the primary fuel for the generation of adenosine triphosphate (ATP) and contractile force in the normal heart. However, they also regulate energy metabolism through peroxisome proliferator-activated receptor- $\alpha$ (PPAR- $\alpha$ ) and its co-activators (Chapter 2). A high fat diet will lead to increased levels of fatty acids (FA) in the blood and drive an increase of FA uptake into the heart. FA can interact with PPARs, which induce upregulation of the expression of proteins and enzymes necessary for their disposal through mitochondrial $\beta$-oxidation, but also stimulate FA uptake. The subsequent lipid overload results in an increased production of reactive oxygen species (ROS) and accumulation of lipid intermediates such as diacylglycerols (DAG) and ceramides. These compounds have a profound impact on signaling pathways, in particular insulin signaling (Chapter 2). As shown in Chapter 7, the hearts of the caPKD mice on a high fat diet did not develop insulin resistance or hypertrophic remodeling, and had a normal lifespan. By studying the mechanism by which high fat diet could overcome hypertrophic remodeling and subsequently prolong the lifespan of these caPKD mice, we noticed that in WT mice on a high fat diet, the PKD protein expression was decreased compared to WT mice on a low fat diet (Chapter 7). Also, in caPKD mice on a high fat diet, the total PKD protein expression level was decreased compared to the caPKD mice on a low fat diet.

The decrease of total PKD protein concentration between the caPKD groups was the same as the decrease observed between the WT groups. These preliminary results indicate that an increase in intracellular lipid levels inhibits PKD expression and as such affects it's downstream signaling. Although the mechanism by which lipids inhibit PKD expression remains to be elucidated, it can be speculated that the nuclear hormone receptor PPAR $\alpha$ and PGC-1 $\alpha$ are involved in this process. PPAR $\alpha$ expression has been shown to be increased during insulin resistance and in type 2 diabetes. ${ }^{69,70}$ Overexpression of the PPAR $\alpha$ isoform in both cardiac and skeletal muscles of mice decreases GLUT4 gene expression and glucose uptake into insulin target tissues. ${ }^{70}$ Mice deficient in PGC- $1 \alpha$ showed a fuel switch towards lipids and developed cardiac dysfunction. ${ }^{71,72}$ Interestingly, also heart-specific HDAC3 KO mice, floxed by Cre under a $\alpha \mathrm{MHC}$-promoter, showed massive cardiac hypertrophy accompanied by up-regulation of genes involved in fatty acid uptake and oxidation, downregulation of glucose utilization, and ligand-induced myocardial lipid accumu- 
lation. ${ }^{73}$ However, HDAC3 KO mice, floxed by a Cre under a muscle creatine kinase promoter, did not exhibit such significant myocardial dysfunction on normal chow, but develop severe cardiomyopathy leading to death when fed a high fat diet. ${ }^{72}$ The difference between those two mouse models is the time point of HDAC3 deletion; in $\mathrm{MHC}^{\mathrm{cre}} / \mathrm{HDAC3}^{\mathrm{fl} / \mathrm{fl}} \mathrm{KO}$ mice, cardiac HDAC3 is already deleted during midgestation, while $\mathrm{MCK}^{\mathrm{cre}} / \mathrm{HDAC}^{\mathrm{fl} / \mathrm{fl}} \mathrm{KO}$ mice have a post-natal cardiac and skeletal deletion of HDAC3. Thus, by using two different promoters, the HDAC3 gene becomes deleted at different time points. The more severe phenotype produced by earlier deletion of HDAC3 suggests that HDAC3 is important during cardiac development. The cardiac changes observed in both mouse models seem to be due to increased activity of PPAR $\alpha^{73}$ In addition, PPAR $\alpha$ is able to downregulate GLUT4 gene expression. Taken in account that HDAC3 may repress PPAR $\alpha$ expression, this could indicate that HDAC3 combines a repression of PPAR $\alpha$ activity with a simultaneously upregulate GLUT4 gene expression. Also, HDAC3 can bind and deacetylate MEF2 and thereby lead to transcriptional repression of MEF2. ${ }^{74}$ Furthermore, it has been shown that HDAC3 co-immunoprecipitates with HDAC5 and is able to interact with MEF2. However, HDAC3 interacts with the MADS box of MEF2, while HDAC5 preferentially bind to the MEF2-specific region. The binding of two HDACs to MEF2 can lead to different conformational changes of MEF2 and will affect the binding efficiency of MEF2 to regulatory elements of genes. The consequence is that binding of HDAC3 to MEF2 will lead to the inhibition of other genes than binding of HDAC5 to MEF2. Therefore, it might be that the binding of HDAC5 to MEF2 leads to inhibition of GLUT4 gene expression and binding of HDAC3 to MEF2 inhibits hypertrophic gene expression. In Chapter 7, we show that PKD is able to activate MEF2, which leads to the expression of GLUT4. Furthermore, is has been shown that MEF2 is able to activate PGC-1 $\alpha$, which also leads to the stimulation of glucose uptake. ${ }^{75}$ Whether HDACs are able to directly bind and repress PGC- $1 \alpha$ activation is not known yet. HDAC5 can be shuttled out of the nucleus upon phosphorylation (by PKD or (aMK) and by binding to 14-3-3, but HDAC3 possesses no obvious sites for 14-3-3 binding. ${ }^{74}$ The signaling cascades which are able to activate HDAC3 remain to be elucidated. However, in my opinion, it could be possible that PKD is able to phosphorylate both HDAC5 and HDAC3 [Fig. 6]. HDAC5 will then bind to 14-3-3 and be shuttled out of the nucleus. HDAC3 possesses no obvious sites for 14-3-3 binding, $^{74}$ and therefore it is unlikely that HDAC3 will shuttle out of the nucleus, but instead phosphorylated HDAC3 may bind to PPAR $\alpha$. In this way, PKD will activate MEF2, which leads to both hypertrophic gene expression and GLUT4 gene expression, but does not induce FA metabolism (may even reduce), because HDAC3 binds to PPAR $\alpha$. Furthermore, it is possible that during PKD activation, MEF2 also induces glucose uptake not only by directly activating the GLUT4 gene, but also by activating PGC-1 $\alpha$. Feeding mice a high fat diet, will lead to the activation of PPAR $\alpha$, which then might inhibit the PKD pathway. When PKD is not active, both HDAC5 and 
HDAC3 do not become phosphorylated and stay bound to MEF2. Thus, a high fat diet leads to increased FA metabolism, and inhibits both glucose metabolism and MEF2-induced hypertrophy. This supports our earlier observations that the hypertrophy developed during a high fat diet is induced by other genes than MEF2 (Chapter 2). Thus, a crosstalk between the HDAC3-PPAR $\alpha$ pathway and the PKD-HDAC5MEF2-GLUT4 pathway could explain why caPKD mice on a high fat diet do not develop insulin resistance and cardiac hypertrophy. For the diabetic heart, the crosstalk between these pathways might provide a link between metabolism and hypertrophic remodeling.

Fig 6 sarcolemma

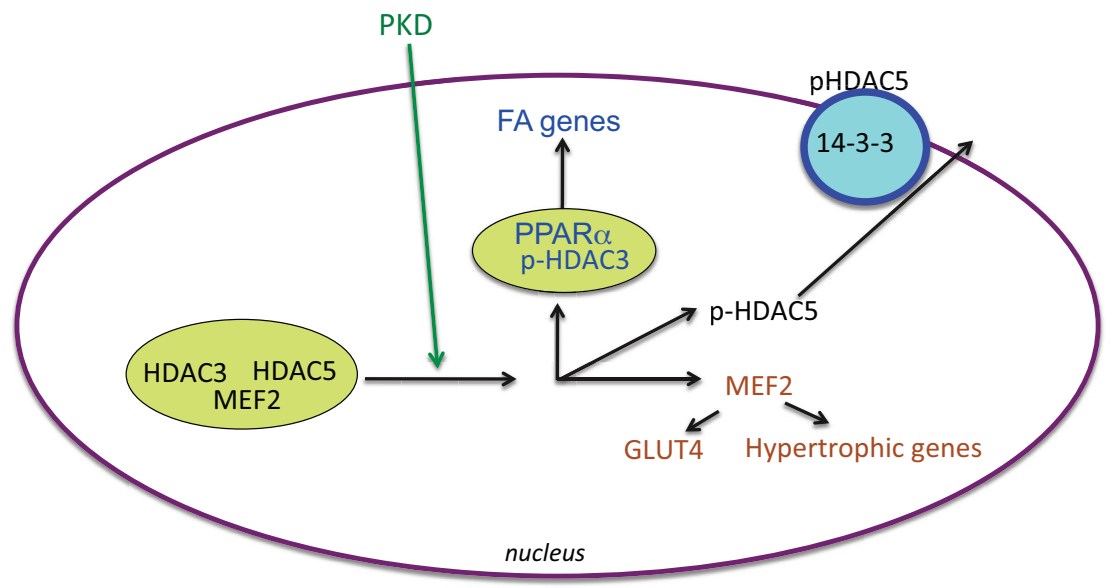

Figure 6: Schematic representation of the hypothesis on PKD induces GLUT4 gene expression and hypertrophic remodeling. When PKD is not activated both HDAC5 and HDAC3 will form a complex with MEF2 to prevent MEF2 activation. Upon PKD activation, both HDAC5 and HDAC3 become phosphorylated. Phosphorylated HDAC5 (pHDAC5) will bind to 14-3-3 and shuttle out of the nucleus, while the released MEF2 will induce hypertrophic genes and the glut4 gene. Phosphorylated HDAC3 (pHDAC3) will bind to PPAR $\alpha$ and thereby inhibit the effect of PPAR $\alpha$ on FA metabolism. Thus, activation of PKD leads to simultaneous induction of hypertrophic genes, increase of GLUT4 concentration and inhibition of FA utilization. 


\section{Future perspectives}

A myriad of pathways is implicated in the development of cardiac hypertrophy. Distinct (sets of) stimuli can activate a variety of signaling cascades and lead to different types of hypertrophies. Physiological hypertrophy does not develop in the same way as pathological hypertrophies do, and comparison of these two remodeling processes and the pathways involved may reveal mechanisms, which specifically lead to pathological hypertrophy. Metabolic aberrations are amongst the triggers that lead to cardiac remodeling and hypertrophy. In this thesis we showed that unbalanced substrate uptake is associated with hypertrophy. Whether it is the cause or the consequence is up for debate. However, based on our results and those published by others, we are inclined to think that unbalanced substrate uptake is a causative factor in cardiac hypertrophic remodeling. If this is the case, then cardiac hypertrophy and heart failure may be prevented by, for instance, special diets. This will not be as simple as it sounds, since this will demand to attune metabolic effects and genetic predisposition. On the diet-side, this thesis clearly shows that cardiac hypertrophy associated with increased FA intake develops differently from hypertrophy associated with increase glucose uptake. On the genetic side, numerous studies have described single nucleotide and other mutations that make patients more vulnerable for a particular hypertrophic pathology. Thus, knowing the patients genome will become more and more relevant to administer an adequate therapy. In this respect, microarrays will become an important diagnostic tool. However, only further research can select and qualify the genes and mutations that are associated with the development of cardiac hypertrophy. 


\section{References}

1. Chottova DM, Slavikova J. [adrenergic regulation of the mammalian heart]. Cesk Fysiol. 2011;60:14-19

2. Triposkiadis F, Karayannis G, Giamouzis G, Skoularigis J, Louridas G, Butler J. The sympathetic nervous system in heart failure physiology, pathophysiology, and clinical implications. J Am Coll Cardiol. 2009;54:1747-1762

3. Bidasee $\mathrm{KR}$, Zheng $\mathrm{H}$, Shao $\mathrm{CH}$, Parbhu SK, Rozanski GJ, Patel KP. Exercise training initiated after the onset of diabetes preserves myocardial function: Effects on expression of betaadrenoceptors. J Appl Physiol. 2008;105:907-914

4. van der Heyden MA, Wijnhoven TJ, Opthof T. Molecular aspects of adrenergic modulation of cardiac I-type ca2+ channels. Cardiovasc Res. 2005;65:28-39

5. Gordon AM, Homsher E, Regnier M. Regulation of contraction in striated muscle. Physiol Rev. 2000;80:853-924

6. Brickson S, Fitzsimons DP, Pereira L, Hacker T, Valdivia H, Moss RL. In vivo left ventricular functional capacity is compromised in cmybp-c null mice. Am J Physiol Heart Circ Physiol. 2007;292:H1747-1754

7. Filtz TM, Grubb DR, McLeod-Dryden TJ, Luo J, Woodcock EA. Gq-initiated cardiomyocyte hypertrophy is mediated by phospholipase cbeta1b. Faseb J. 2009;23:3564-3570

8. Phan D, Stratton MS, Khai Huynh $Q$, McKinsey TA. A novel protein kinase c target site in protein kinase $d$ is phosphorylated in response to signals for cardiac hypertrophy. Biochem Biophys Res Commun. 2011;411:335-341

9. Haworth RS, Roberts NA, Cuello F, Avkiran M. Regulation of protein kinase d activity in adult myocardium: Novel counter-regulatory roles for protein kinase cepsilon and protein kinase a. $J$ Mol Cell Cardiol. 2007;43:686-695

10. Bardswell SC, Cuello F, Rowland AJ, Sadayappan S, Robbins J, Gautel M, Walker JW, Kentish JC, Avkiran M. Distinct sarcomeric substrates are responsible for protein kinase d-mediated regulation of cardiac myofilament ca2+ sensitivity and cross-bridge cycling. J Biol Chem. 2010;285:5674-5682

11. Kooij V, Boontje N, Zaremba R, Jaquet K, dos Remedios C, Stienen GJ, van der Velden J. Protein kinase $\mathrm{c}$ alpha and epsilon phosphorylation of troponin and myosin binding protein c reduce ca2+ sensitivity in human myocardium. Basic Res Cardiol. 2010;105:289-300

12. Chen PP, Patel JR, Rybakova IN, Walker JW, Moss RL. Protein kinase a-induced myofilament desensitization to $\mathrm{ca}(2+)$ as a result of phosphorylation of cardiac myosin-binding protein $\mathrm{c}$. $J$ Gen Physiol. 2010;136:615-627

13. Wong W, Scott JD. Akap signalling complexes: Focal points in space and time. Nat Rev Mol Cell Biol. 2004;5:959-970

14. Fraser ID, Tavalin SJ, Lester LB, Langeberg LK, Westphal AM, Dean RA, Marrion NV, Scott JD. A novel lipid-anchored a-kinase anchoring protein facilitates camp-responsive membrane events. Embo J. 1998;17:2261-2272

15. Carnegie GK, Smith FD, McConnachie G, Langeberg LK, Scott JD. Akap-lbc nucleates a protein kinase d activation scaffold. Mol Cell. 2004;15:889-899

16. Carnegie GK, Means CK, Scott JD. A-kinase anchoring proteins: From protein complexes to physiology and disease. IUBMB Life. 2009;61:394-406

17. Li L, He Q, Gao YS. [phosphodiesterase regulation of cardiovascular functions]. Sheng Li Ke Xue Jin Zhan. 2010;41:100-106

18. Dodge KL, Khouangsathiene S, Kapiloff MS, Mouton R, Hill EV, Houslay MD, Langeberg LK, Scott JD. Makap assembles a protein kinase a/pde4 phosphodiesterase camp signaling module. Embo J. 2001;20:1921-1930

19. Zaccolo M, Pozzan T. Discrete microdomains with high concentration of camp in stimulated rat neonatal cardiac myocytes. Science. 2002;295:1711-1715

20. Palmer D, Jimmo SL, Raymond DR, Wilson LS, Carter RL, Maurice DH. Protein kinase a phosphorylation of human phosphodiesterase $3 \mathrm{~b}$ promotes 14-3-3 protein binding and inhibits phosphatase-catalyzed inactivation. J Biol Chem. 2007;282:9411-9419 
21. McConnachie G, Langeberg LK, Scott JD. Akap signaling complexes: Getting to the heart of the matter. Trends Mol Med. 2006;12:317-323

22. Masciarelli S, Horner K, Liu C, Park SH, Hinckley M, Hockman S, Nedachi T, Jin C, Conti M, Manganiello V. Cyclic nucleotide phosphodiesterase 3a-deficient mice as a model of female infertility. J Clin Invest. 2004;114:196-205

23. Wong AK, Howie J, Petrie JR, Lang CC. Amp-activated protein kinase pathway: A potential therapeutic target in cardiometabolic disease. Clin Sci (Lond). 2009;116:607-620

24. Andersson DC, Fauconnier J, Yamada T, Lacampagne A, Zhang SJ, Katz A, Westerblad H. Mitochondrial production of reactive oxygen species contributes to the beta-adrenergic stimulation of mouse cardiomycytes. J Physiol. 2011;589:1791-1801

25. Coort SL, Bonen A, van der Vusse GJ, Glatz JF, Luiken JJ. Cardiac substrate uptake and metabolism in obesity and type-2 diabetes: Role of sarcolemmal substrate transporters. Mol Cell Biochem. 2007;299:5-18

26. Habets DD, Coumans WA, El Hasnaoui M, Zarrinpashneh E, Bertrand L, Viollet B, Kiens B, Jensen TE, Richter EA, Bonen A, Glatz JF, Luiken JJ. Crucial role for Ikb1 to ampkalpha2 axis in the regulation of cd36-mediated long-chain fatty acid uptake into cardiomyocytes. Biochim Biophys Acta. 2009;1791:212-219

27. Shisheva A. Phosphoinositides in insulin action on glut4 dynamics: Not just ptdins $(3,4,5)$ p3. Am J Physiol Endocrinol Metab. 2008;295:E536-544

28. Hausser A, Storz P, Martens S, Link G, Toker A, Pfizenmaier K. Protein kinase d regulates vesicular transport by phosphorylating and activating phosphatidylinositol-4 kinase iiibeta at the golgi complex. Nat Cell Biol. 2005;7:880-886

29. Kristiansen S, Ramlal T, Klip A. Phosphatidylinositol 4-kinase, but not phosphatidylinositol 3kinase, is present in glut4-containing vesicles isolated from rat skeletal muscle. Biochem J. 1998;335 ( Pt 2):351-356

30. Demmel L, Beck M, Klose C, Schlaitz AL, Gloor Y, Hsu PP, Havlis J, Shevchenko A, Krause E, Kalaidzidis $Y$, Walch-Solimena $C$. Nucleocytoplasmic shuttling of the golgi phosphatidylinositol 4-kinase pik1 is regulated by 14-3-3 proteins and coordinates golgi function with cell growth. Mol Biol Cell. 2008;19:1046-1061

31. Pozuelo Rubio M, Geraghty KM, Wong BH, Wood NT, Campbell DG, Morrice N, Mackintosh C. 14-3-3-affinity purification of over 200 human phosphoproteins reveals new links to regulation of cellular metabolism, proliferation and trafficking. Biochem J. 2004;379:395-408

32. Dorner C, Ullrich A, Haring HU, Lammers R. The kinesin-like motor protein kif1c occurs in intact cells as a dimer and associates with proteins of the 14-3-3 family. J Biol Chem. 1999;274:33654-33660

33. Ichimura T, Wakamiya-Tsuruta A, Itagaki C, Taoka M, Hayano T, Natsume T, Isobe T. Phosphorylation-dependent interaction of kinesin light chain 2 and the 14-3-3 protein. Biochemistry. 2002;41:5566-5572

34. van Heusden GP. 14-3-3 proteins: Insights from genome-wide studies in yeast. Genomics. 2009;94:287-293

35. Shikano S, Coblitz B, Wu M, Li M. 14-3-3 proteins: Regulation of endoplasmic reticulum localization and surface expression of membrane proteins. Trends Cell Biol. 2006;16:370-375

36. Nishino TG, Miyazaki M, Hoshino H, Miwa Y, Horinouchi S, Yoshida M. 14-3-3 regulates the nuclear import of class iia histone deacetylases. Biochem Biophys Res Commun. 2008;377:852856

37. Grozinger CM, Schreiber SL. Regulation of histone deacetylase 4 and 5 and transcriptional activity by 14-3-3-dependent cellular localization. Proc Natl Acad Sci U S A. 2000;97:7835-7840

38. Carnegie GK, Soughayer J, Smith FD, Pedroja BS, Zhang F, Diviani D, Bristow MR, Kunkel MT, Newton AC, Langeberg LK, Scott JD. Akap-lbc mobilizes a cardiac hypertrophy signaling pathway. Mol Cell. 2008;32:169-179

39. McGee SL, van Denderen BJ, Howlett KF, Mollica J, Schertzer JD, Kemp BE, Hargreaves M. Amp-activated protein kinase regulates glut4 transcription by phosphorylating histone deacetylase 5. Diabetes. 2008;57:860-867

40. Ha CH, Kim JY, Zhao J, Wang W, Jhun BS, Wong C, Jin ZG. Pka phosphorylates histone deacetylase 5 and prevents its nuclear export, leading to the inhibition of gene transcription and cardiomyocyte hypertrophy. Proc Natl Acad Sci U S A. 2010;107:15467-15472 
41. Ro A, Frishman WH. Peripartum cardiomyopathy. Cardiol Rev. 2006;14:35-42

42. Dobrin JS, Lebeche D. Diabetic cardiomyopathy: Signaling defects and therapeutic approaches. Expert Rev Cardiovasc Ther. 2010;8:373-391

43. Kacimi R, Gerdes AM. Alterations in g protein and map kinase signaling pathways during cardiac remodeling in hypertension and heart failure. Hypertension. 2003;41:968-977

44. Mao J, Yuan H, Xie W, Simon MI, Wu D. Specific involvement of g proteins in regulation of serum response factor-mediated gene transcription by different receptors. J Biol Chem. 1998;273:27118-27123

45. Adams JW, Brown JH. G-proteins in growth and apoptosis: Lessons from the heart. Oncogene. 2001;20:1626-1634

46. Miyamoto S, Del Re DP, Xiang SY, Zhao X, Florholmen G, Brown JH. Revisited and revised: Is rhoa always a villain in cardiac pathophysiology? J Cardiovasc Transl Res. 2010;3:330-343

47. Diviani D, Baisamy L, Appert-Collin A. Akap-lbc: A molecular scaffold for the integration of cyclic amp and rho transduction pathways. Eur J Cell Biol. 2006;85:603-610

48. Glass DJ. Pi3 kinase regulation of skeletal muscle hypertrophy and atrophy. Curr Top Microbiol Immunol. 2010;346:267-278

49. Vega RB, Harrison BC, Meadows E, Roberts CR, Papst PJ, Olson EN, McKinsey TA. Protein kinases $\mathrm{C}$ and $\mathrm{d}$ mediate agonist-dependent cardiac hypertrophy through nuclear export of histone deacetylase 5. Mol Cell Biol. 2004;24:8374-8385

50. Cuello F, Bardswell SC, Haworth RS, Yin X, Lutz S, Wieland T, Mayr M, Kentish JC, Avkiran M. Protein kinase $\mathrm{d}$ selectively targets cardiac troponin $\mathrm{i}$ and regulates myofilament ca2+ sensitivity in ventricular myocytes. Circ Res. 2007;100:864-873

51. Storz $P$, Toker A. Protein kinase $d$ mediates a stress-induced nf-kappab activation and survival pathway. Embo J. 2003;22:109-120

52. Storz P, Doppler H, Toker A. Protein kinase d mediates mitochondrion-to-nucleus signaling and detoxification from mitochondrial reactive oxygen species. Mol Cell Biol. 2005;25:85208530

53. Huynh QK, McKinsey TA. Protein kinase d directly phosphorylates histone deacetylase 5 via a random sequential kinetic mechanism. Arch Biochem Biophys. 2006;450:141-148

54. Chang VK, Donato JJ, Chan CS, Tye BK. Mcm1 promotes replication initiation by binding specific elements at replication origins. Mol Cell Biol. 2004;24:6514-6524

55. Song K, Backs J, McAnally J, Qi X, Gerard RD, Richardson JA, Hill JA, Bassel-Duby R, Olson EN. The transcriptional coactivator camta2 stimulates cardiac growth by opposing class ii histone deacetylases. Cell. 2006;125:453-466

56. Fielitz J, Kim MS, Shelton JM, Qi X, Hill JA, Richardson JA, Bassel-Duby R, Olson EN. Requirement of protein kinase d1 for pathological cardiac remodeling. Proc Natl Acad Sci U $S$ A. 2008;105:3059-3063

57. Harrison BC, Kim MS, van Rooij E, Plato CF, Papst PJ, Vega RB, McAnally JA, Richardson JA, Bassel-Duby R, Olson EN, McKinsey TA. Regulation of cardiac stress signaling by protein kinase d1. Mol Cell Biol. 2006;26:3875-3888

58. Passier R, Zeng H, Frey N, Naya FJ, Nicol RL, McKinsey TA, Overbeek P, Richardson JA, Grant $\mathrm{SR}$, Olson EN. Cam kinase signaling induces cardiac hypertrophy and activates the mef2 transcription factor in vivo. J Clin Invest. 2000;105:1395-1406

59. Appert-Collin A, Cotecchia S, Nenniger-Tosato M, Pedrazzini T, Diviani D. The a-kinase anchoring protein (akap)-lbc-signaling complex mediates alpha1 adrenergic receptor-induced cardiomyocyte hypertrophy. Proc Natl Acad Sci U S A. 2007;104:10140-10145

60. Mayers CM, Wadell J, McLean K, Venere M, Malik M, Shibata T, Driggers PH, Kino T, Guo XC, Koide H, Gorivodsky M, Grinberg A, Mukhopadhyay M, Abu-Asab M, Westphal H, Segars JH. The rho guanine nucleotide exchange factor akap13 (brx) is essential for cardiac development in mice. J Biol Chem. 2010;285:12344-12354

61. Aye TT, Soni S, van Veen TA, van der Heyden MA, Cappadona S, Varro A, de Weger RA, de Jonge N, Vos MA, Heck AJ, Scholten A. Reorganized pka-akap associations in the failing human heart. J Mol Cell Cardiol. 2011

62. Zhao JX, Yue WF, Zhu MJ, Du M. Amp-activated protein kinase regulates beta-catenin transcription via histone deacetylase 5. J Biol Chem. 2011;286:16426-16434 
63. Gong H, Xie J, Zhang N, Yao L, Zhang Y. Mef2a binding to the glut4 promoter occurs via an ampkalpha2-dependent mechanism. Med Sci Sports Exerc. 2011;43:1441-1450

64. Kim J, Wende AR, Sena S, Theobald HA, Soto J, Sloan C, Wayment BE, Litwin SE, Holzenberger $M$, LeRoith $D$, Abel ED. Insulin-like growth factor i receptor signaling is required for exerciseinduced cardiac hypertrophy. Mol Endocrinol. 2008;22:2531-2543

65. Lehnen AM, Leguisamo NM, Pinto GH, Markoski M, De Angelis K, Machado UF, Schaan B. Exercise-stimulated glut4 expression is similar in normotensive and hypertensive rats. Horm Metab Res. 2011;43:231-235

66. Kim HJ, Lee JS, Kim CK. Effect of exercise training on muscle glucose transporter 4 protein and intramuscular lipid content in elderly men with impaired glucose tolerance. Eur J Appl Physiol. 2004;93:353-358

67. Smith JA, Kohn TA, Chetty AK, Ojuka EO. Camk activation during exercise is required for histone hyperacetylation and mef2a binding at the mef2 site on the glut4 gene. Am J Physiol Endocrinol Metab. 2008;295:E698-704

68. McGee SL. Exercise and mef2-hdac interactions. Appl Physiol Nutr Metab. 2007;32:852-856

69. Duncan JG. Peroxisome proliferator activated receptor-alpha (pparalpha) and ppar gamma coactivator-1alpha (pgc-1alpha) regulation of cardiac metabolism in diabetes. Pediatr Cardiol. 2011;32:323-328

70. Lecarpentier Y, Claes V, Hebert JL. Ppars, cardiovascular metabolism, and function: Near- or far-from-equilibrium pathways. PPAR Res. 2010;2010

71. Liang H, Ward WF. Pgc-1alpha: A key regulator of energy metabolism. Adv Physiol Educ. 2006;30:145-151

72. Sun Z, Singh N, Mullican SE, Everett LJ, Li L, Yuan L, Liu X, Epstein JA, Lazar MA. Diet-induced lethality due to loss of hdac3 in heart and skeletal muscle. J Biol Chem. 2011

73. Montgomery RL, Potthoff MJ, Haberland M, Qi X, Matsuzaki S, Humphries KM, Richardson JA, Bassel-Duby R, Olson EN. Maintenance of cardiac energy metabolism by histone deacetylase 3 in mice. J Clin Invest. 2008;118:3588-3597

74. Gregoire S, Xiao L, Nie J, Zhang X, Xu M, Li J, Wong J, Seto E, Yang XJ. Histone deacetylase 3 interacts with and deacetylates myocyte enhancer factor 2. Mol Cell Biol. 2007;27:1280-1295

75. Czubryt MP, McAnally J, Fishman GI, Olson EN. Regulation of peroxisome proliferatoractivated receptor gamma coactivator 1 alpha (pgc-1 alpha ) and mitochondrial function by mef2 and hdac5. Proc Natl Acad Sci U S A. 2003;100:1711-1716 
Summary

Samenvatting 


\section{Summary}

Changes in metabolism, contractility or morphology contribute to the development of cardiomyopathy and heart failure. These changes are the result of signals from the body to the heart, which then induce cellular signaling cascades. A signaling cascade is a succession of amplifying intracellular reactions, which starts with the activation of a membrane receptor, followed by activation of other proteins. Kinases are frequently part of signaling cascades, and forward the signal through phosphorylation of target proteins. Protein kinase $D(P K D)$ is such a protein. In the beginning of this study, there were indications that PKD plays a role in signaling cascades involved in glucose uptake and the regulation of contraction, and of development of hypertrophy of heart muscle. However, the link and underlying mechanism(s) of these processes were barely known.

One of the diseases in which the link between metabolism, contractility and morphology is disturbed, is type 2 diabetes. In the diabetic heart, there is an increased uptake of fatty acids, which initially is a compensation for impaired glucose uptake (due to insulin resistance) to safeguard the availability of sufficient substrates for energy production. Ultimately this substrate switch leads to cardiac hypertrophy and dysfunction of heart muscle. An imbalance of cardiac substrate uptake (in this case a relatively high fatty acid uptake at the cost of glucose uptake) is known eventually to lead to heart failure. Based on this, it could be suggested that stimulation of glucose uptake would restore the substrate balance of the diabetic heart where after hypertrophy and heart failure will be restored. Thus, stimulation of (part of) a signaling cascade which specifically increases glucose uptake without inducing fatty acid uptake could be a useful therapy to prevent heart failure in diabetic patients. Previously, AMPK has been extensively studied in this context. Unfortunately, activation of AMPK stimulates the uptake of both glucose and fatty acids and, therefore, AMPK activation is not able to prevent diabetic cardiomyopathy. Preliminary data (using pharmacological agents) indicated that PKD is involved in the translocation of the membrane transporter GLUT4 to the plasma membrane, which is a necessary step to allow cellular glucose uptake. However, the effect of PKD activation on fatty acid uptake had not yet been investigated.

In this study, we started to investigate the role of PKD in cardiac substrate uptake by using cells in culture. In these cultured cells, PKD appeared to stimulate glucose uptake but not fatty acid uptake. By using isolated rat cardiomyocytes, we were able to map part of the pathway, which leads to activation of PKD and PKD-mediated glucose uptake. Specifically, we identified the binding of DAP-kinase to PKD upon contraction-inducing radical production. This binding leads to the activation of PKD, where after GLUT4 translocates to the plasma membrane to facilitate glucose uptake (Chapter $\mathbf{3}$ ). Additionally, we 
showed that PKD and AMPK function in parallel pathways, but both lead to glucose uptake. Later on, we were able to validate these in vitro data in intact animals by using microPET imaging of hearts from transgenic mice overexpressing PKD in cardiac muscle (caPKD mice). From these in vitro and in vivo observations we thus could conclude that PKD is able to increase glucose uptake, without stimulating fatty acid uptake.

Does this suggest that PKD can restore the substrate balance in the diabetic heart? One of the features of the pre-diabetic heart is a decreased insulin sensitivity. Therefore, both PKD and - for comparison - AMPK were studied in cardiomyocytes exposed to culture media with elevated insulin or palmitate concentrations so as to make the cells less insulin sensitive. It was found that both PKD overexpression and AMPK overexpression prevented the decrease in insulin sensitivity. Additionally, it became clear (although this effect was not significant) that PKD overexpression, but not AMPK overexpression, could decrease the fat storage (induced by increased levels of palmitate in the culture medium) in cardiomyocytes (chapter 6). From these combined data we conclude that PKD overexpression, but not AMPK overexpression, prevents both excessive fat storage and decreased insulin sensitivity in a cell model of high-fat induced cardiac dysfunction.

Besides the metabolic aberrations, the diabetic heart also has to cope with a decreased contraction. It was previously published that PKD affects the contractility of heart muscle by phosphorylating sarcomeric proteins. However, it was unknown which proteins are phosphorylated and what the functional relevance is of these PKD-mediated phosphorylations. In chapter 5, we demonstrated that PKD is able to phosphorylate cMyBP-C. By using cMyBP-C knock-out mice, we showed that this protein is part of the contractile apparatus and upon phosphorylation strengthens the contraction. The latter effect is important especially during increased contraction-frequency, when the demand for energy is highest. This observation suggests that PKD act as a link between cardiac metabolism and contractility. In that case, PKD would operate as a mediator, which during increased activity of the heart strengthens both the contractility and the uptake of glucose so as to provide the contracting cells of sufficient fuel for energy production.

From literature it is known that an increased glucose uptake is associated with hypertrophy and heart failure. Assuming the hypothesis that a balanced substrate uptake is essential for normal heart function, hypertrophy associated with an increased glucose uptake might be counterbalanced and/or prevented by increasing fatty acid uptake. In chapter $\mathbf{7}$ we demonstrated that this indeed is the case. CaPKD mice display hypertrophy associated with an increased glucose uptake, and these mice die at a young age (less than 6 months old). Moreover, by using wild-type mice on a high fat diet, we showed that also 
increased fatty acid uptake is associated with hypertrophic remodeling. These wild type mice on a high fat diet developed decreased insulin sensitivity and displayed cardiac hypertrophy. In contrast, feeding caPKD mice a high fat diet restored cardiac substrate uptake (similar contributions from glucose and fatty acids) and insulin sensitivity As a result, the hypertrophy of the caPKD mice was significantly less than caPKD mice on a low fat diet while they had a natural life span. Taken together, these data convincingly underscore that a balanced substrate uptake is crucial for the normal functioning of the heart.

Because caPKD mice displayed both increased cardiac glucose uptake and cardiac hypertrophy, it became plausible that PKD connects cardiac hypertrophy and metabolism. In chapter 7, we demonstrated that PKD induces activation of the transcription factor MEF2D. MEF2D is one of the most prominent transcription factors involved in the regulation of both the GLUT4 gene and a group of genes associated with hypertrophic remodeling.

The findings described in this thesis emphasize the importance of integral studies for the functioning of the heart. Signaling proteins, like kinases, appear able to function in or connect several pathways. Linking such pathways contributes to the overall picture of how these processes are controlled in a cardiomyocyte. This thesis shows the role of PKD in metabolism, contractility and cell growth, and how these processes could influence each other. In this way, PKD can stimulate the functional association of independent processes, however this seems to happen in concert with other factors (chapter 8). Thus, it is the integration and communication of several pathways, which is important. This especially points to multifactorial diseases like diabetic cardiomyopathy, by which defective functioning processes are often dependent on several signaling cascades. Therefore, scientists should try to broaden their view and to explore interdisciplinary borders in order to overlook the overall picture. 


\section{Samenvatting}

Veranderingen in zowel metabolisme, contractiliteit als morfologie dragen bij aan de ontwikkeling van cardiomypathie en hartfalen. Deze veranderingen zijn het gevolg van signalen vanuit het lichaam die het hart bereiken en bepaalde signaleringscascades in gang zetten. Een signaleringscascade is een serie elkaar versterkende, intracellulaire reacties die start met de activering van een membraan receptor en vervolgens wordt doorgegeven aan intracellulaire eiwitten of eiwitcomplexen. Kinases zijn vaak onderdeel van zo'n signaleringscascade en geven het signaal door via fosforylering van andere eiwitten. Protein kinase D (PKD) is zulk een eiwit. Bij de aanvang van deze studie waren er aanwijzingen dat PKD een rol speelt in cascades betrokken bij de cellulaire glucose opname en de regulatie van contractiliteit en van hypertrofie van de hartspier. Echter, het verband en de mechanismen van deze processen waren nauwelijks gekend.

Eén van de ziektes waarbij het verband tussen deze processen van belang is, is type 2 diabetes. In het diabete hart is er een verhoogde opname van vetzuren waardoor er hypertrofie en dysfunctie van de hartspier ontstaat. Deze onevenwichtigheid in cardiale substraat opname (relatief veel vetzuren en relatief weinig glucose) zal uiteindelijk leiden tot hartfalen. Op basis hiervan zou men kunnen veronderstellen dat specifieke stimulatie van de glucose opname de substraat balans in het diabete hart weer in evenwicht kan brengen, waardoor hypertrofie en hartfalen tegengegaan danwel voorkomen kan worden. Indien we dus een (onderdeel van een) signaleringscascade zouden kunnen vinden die uitsluitend de glucose opname stimuleert zonder de vetzuur opname te bevorderen, zou dat kunnen leiden tot een nieuwe therapie voor diabete patiënten die lijden aan hartfalen.

Eerder is in deze context AMPK uitgebreid bestudeerd. Echter, AMPK activatie leidt tot zowel een verhoogde opname van glucose als van vetzuren, waardoor activatie van AMPK uiteindelijk niet in staat is de ontwikkeling van diabete cardiomyopathie tegen te gaan. In verkennende studies (met farmacologische stoffen) was gevonden dat PKD betrokken is bij de verplaatsing van de glucose transporter GLUT4 naar de plasmamembraan, nodig voor de opname van glucose. Echter, de effecten van PKD op de vetzuur opname waren niet bekend. Daarom hebben we eerst in gekweekte cellen uitgezocht wat de rol is van PKD bij de substraat opname. PKD bleek de glucose opname te stimuleren, maar niet de vetzuur opname. En passant brachten we een deel van de signaleringscascade in kaart die leidt tot activatie van PKD en een PKDaangestuurde glucose opname. We ontdekten een binding van DAP-kinase aan PKD die het gevolg is van contractie-gegenereerde radicalen. Deze binding leidt tot de activatie van PKD, waardoor GLUT4 naar de plasmamembraan kan worden verplaatst om glucose op te nemen (hoofdstuk 3). Verder hebben we 
gevonden dat PKD en AMPK parallel functioneren, maar wel allebei leiden tot glucose opname. Deze, in een cellijn, gemeten effecten van PKD op de cardiale substraat opname werden bevestigd in geïsoleerde hartspiercellen. Later bleken deze resultaten ook te kloppen toen gebruik kon worden gemaakt van in vivo microPET beeldvorming in harten van transgene muizen met een overmaat aan productie van PKD (caPKD muizen).

Eén van de kenmerken van het diabete hart is een verminderde insulinegevoeligheid. In een experimenteel model van hartspiercellen met een verminderde insulingevoeligheid (verkregen door te cellen te kweken in een medium met een verhoogde hoeveelheid insulin of palmitaat) de werking van AMPK en PKD bestudeerd. Door gebruik te maken van dit model is gebleken dat zowel een overmaat aan PKD als van AMPK de vermindering in insulinegevoeligheid kan tegengaan. Ook werd duidelijk dat PKD overexpressie, maar niet AMPK overexpressie de vetstapeling in de hartspiercellen (geïnduceerd door een verhoogde dosis palmitaat in het medium) kan tegengaan (hoofdstuk 6).

Uit de bovenstaande gegevens concluderen we dat PKD de glucose opname stimuleert zonder de opname vetzuren te bevorderen. Naast de metabole afwijkingen heeft het diabete hart ook te maken met verminderde contractie. Van PKD was al bekend dat het effect zou hebben op de contractiliteit van de hartspier door fosforylatie van sarcomere eiwitten, maar het was onbekend welke sarcomere eiwitten door PKD worden gefosforyleerd en wat de functionele relevantie hiervan is. In hoofdstuk $\mathbf{4}$ is gebruik gemaakt van cMyBP$C$ knock-out muizen, en laten we zien dat PKD in staat is om het eiwit cMyBP-C te fosforyleren. Dit eiwit maakt deel uit van het contractiele apparaat van de hartspier. Door deze fosforylatie neemt de kracht van de contractie toe en dit is van belang bij een verhoogde contractie-frequentie, wanneer de vraag naar energie steeds groter wordt. Dit doet ons veronderstellen dat PKD een verband legt tussen het cardiale metabolisme en contractiliteit. PKD dient dan als een mediator die bij grotere activiteit van het hart enerzijds de contractiliteit versterkt en anderzijds - door de glucose opname te stimuleren - ervoor zorgt dat de cel genoeg energie kan produceren.

Uit de literatuur is gebleken dat een overmaat aan glucose opname ook geassocieerd is met hypertropfie en hartfalen. Uitgaande van de stelling dat een evenwichtige substraatopname essentieel is voor het normaal functioneren van het hart, zou in het geval van hypertrofie geassocieerd met een verhoogde glucose opname, een verhoogde vetzuur opname deze hypertrofie kunnen tegengaan. In hoofdstuk 7 tonen we aan dat dit inderdaad het geval is. caPKD muizen vertonen hypertrofie geassocieerd met een verhoogde glucose opname, en daardoor sterven deze dieren jong (levensverwachting minder dan een half jaar). Daarentegen ontwikkelen wild-type muizen op een hoge vet voeding een 
verminderde insulinegevoeligheid en vertonen ze cardiale hypertrofie, maar hebben wel een redelijk normale levensverwachting. Wanneer caPKD muizen op een hoog vet voeding worden gezet bleek de insulinegvoeligheid en de morfologie van het hart niet afwijkend van de controlegroep. Door deze muizen een hoog vet voeding te geven wordt de cardiale substraatopname weer gebalanceerd (opname van vergelijkbare hoeveelheden glucose en vetzuren) en de hypertrofie significant verminderd. Dit ging samen met een normale levensverwachting. Dit alles wijst er op dat de substraatbalans van cruciaal belang is bij het goed functioneren van het hart.

Omdat caPKD muizen zowel een verhoogde cardiale glucose opname als hypertrofie vertonen, leek het ons aannemelijk dat PKD ook de cardiale hypertrofie en het metabolisme verbindt. In hoofdstuk 7 tonen we aan dat PKD activatie van de transcriptiefactor MEF2D induceert. MEF2D is een van de belangrijkste transcriptiefactoren betrokken bij de regulatie van zowel het GLUT4 gen alsook van een grote groep genen betrokken bij hypertrofie. De experimentele hoofdstukken van dit proefschrift laten zien dat aansturing van cardiale contractie, metabolisme en hypertrofie voor een deel door PKD gebeurt. Om de rol van PKD in perspectief te plaatsen, laten we in hoofdstuk 8 zien dat dit slechts een klein facet is van de regelsystemen die deze processen besturen.

De in de experimentele hoofdstukken beschreven bevindingen onderstrepen het belang van integrale studies naar het functioneren van het hart. Men kan dit benaderen door het gebruik van arrays maar ook door onderzoek naar het functioneren van een eiwit dat bij meerdere processen betrokken is. Signaaleiwitten zoals kinases blijken in verschillende signaalcascades te kunnen functioneren of deze te kunnen verbinden. Het samenvoegen van dergelijke cascades draagt bij tot het totaalbeeld van hoe de processen in een cardiomyocyt worden aangestuurd. De in dit proefschrift beschreven studies laten zien welke rol PKD speelt in zowel het metabolisme, de contractiliteit als in de celgroei en hoe deze processen elkaar kunnen beïnvloeden. Op deze manier kan PKD de functionele samenhang van onafhankelijke processen bevorderen, al blijkt dat in samenspraak met veel andere factoren te moeten gebeuren (hoofdstuk 8). De integratie en communicatie van verschillende signaalcascades blijkt dus van belang. Dit geldt zeker voor multifactoriele aandoeningen, zoals diabete cardiomyopathy, waar gebrekkig functionerende processen vaak afhankelijk zijn van meerdere signaleringscascades. In dit soort studies moeten wetenschappers proberen hun blik te verruimen en interdiciplinaire grenzen af te tasten om zo vat te krijgen op het geheel. 
Dankwoord 


\section{Dankwoord}

Het was een periode van hard werken, waar geluk en tegenslag mekaar afwisselden, maar ik ben ontzettend blij met het resultaat. Ik heb het gevoel dat deze periode me enorm verrijkt heeft en ben dan ook enorm fier op het werk wat in dit proefschrift beschreven staat. Uiteraard heb ik dit niet enkel aan mezelf te danken, maar aan iedereen die me gedurende de afgelopen vier jaar gesteund en geholpen hebben. Zonder jullie had ik nu niet gestaan waar ik nu sta!

Als allereerste wil ik graag mijn twee copromotoren, Joost en Guillaume, bedanken. Joost, vanaf onze eerste kennismaking was jij diegene die in mij geloofde, en ben ik dan ook kunnen beginnen als PhD studente op Moleculaire Genetica. Gedurende de eerste periode heb ik onder jouw begeleiding mij een weg gemaakt in het lab, en doordat jij altijd achter mij stond, heb ik mij kunnen ontplooien tot een zelfstandig onderzoeker. Daarom wil ik je bedanken dat jij mijn copromotor was!

Guillaume, jij verscheen op het toneel vanaf het moment dat ik met de muismodellen aan de slag ging. Je bent voor mij een enorme steun geweest en ik heb van jou zowel op wetenschappelijk als persoonlijk vlak enorm veel bijgeleerd. Dankzij jou ben ik gaan inzien dat je als wetenschapper een interdisciplinaire kijk op de zaak moet hebben. Ik heb vele dagen versleten op die ene stoel bij u op de kamer, waar we uren hebben gediscussieerd over nieuwe ideeën voor experimenten en waarna we dan natuurlijk sowieso afdwaalden om nog even te leuteren over het nieuwe appartement of uw groentetuin. :) Het ging misschien niet altijd even efficiënt, maar alle gesprekken waren wel zeker relevant en daarom ben ik ook ontzettend blij dat jij mijn copromotor was!

Beste Jan, bedankt om promotor te zijn van mijn PhD project. Dankzij jou heb ik de kans gehad om mijn werk op verschillende congressen te mogen presenteren. Al in mijn eerste PhD jaar, stond ik als groentje op het SHVM congres in Boston. Dit was voor mij echt een geweldige ervaring! Ook alle andere keren dat ik mijn werk heb mogen presenteren, hebben enerzijds mijn presentatie-skills enorm opgekrikt en anderzijds mij de kans gegeven om mijn netwerken uit te breiden. Ook heb je me de financiële mogelijkheden gegeven om mijn studie op een hoger niveau te brengen (o.a. door de microPET scans). Bedankt!

Robert, jij was de postdoc in ons lab waar ik enorm naar opkeek! Ook jij hebt me enorm veel bijgeleerd op zowel wetenschappelijk als persoonlijk vlak. Samen hebben we uren in de celkweek doorgebracht en vond het ook altijd heel plezierig om met u samen te werken! Je deur stond altijd open en je hebt 
me ook enorm gesteund. Vandaar geen twijfel mogelijk om jou als paranimf te vragen!

Veerle, ik ken je al van de eerste dag in diepenbeek. En vooral de periode dat we samen op kot zaten, was echt geweldig! Ik ben misschien wel altijd de rebel geweest van ons twee, maar ik had toch enorm veel chance met zo'n vriendin als jij, die me op tijd verwittigde om naar de les te gaan en die me liet inzien dat een beetje structuur in je cursus geen kwaad kon (dan denk ik voornamelijk ook aan ons eerste open-boek examen)... (:) Toen ik mijn eerste werkdag op mijn kamer aankwam, was ik eerst enorm verbaasd, maar ook zeker aangenaam verrast dat jij daar ook zat! Ook gedurende onze PhD-periode ben je er altijd voor mij geweest en daarom was het voor mij ook vanzelfsprekend om jou als paranimf te vragen!

Nicole, Will en Mohammed, ook jullie wil ik graag bedanken voor alle technische steun. Nicole, ik zou echt niet weten hoeveel cardiomyocyten isolaties jij voor mij hebt gedaan!! (;) Het is ongelofelijk! Maar naast je technische steun, was je ook een hele gezellige collega en vond het daarom altijd plezierig om met jou in het c-lab te staan! Vond het ook super dat je zo begaan was met de resultaten en dat ik bij jou altijd steun kon vinden! Bedankt!!! Mo, ik heb maar gedurende een korte periode met jou samengewerkt, maar heb me super geamuseerd met $u$ ! Jij hebt me gedurende de eerste maanden ook van alle technieken geleerd en vond het erg leuk samen met jou in het lab te staan. Will, je kon hard vloeken; woorden als "eikel" en "daar krijg ik een punthoofd van" zal ik niet snel vergeten. :) Maar, je hebt me ook verschillende keren uit de nood geholpen door western blots voor me te draaien, en daar ben ik je heel dankbaar voor!

Vervolgens zou ik ook al mijn (ex)-kamergenootjes willen bedanken. We hadden echt een super gezellige AlO-kamer, wat het werken dan ook zeer aangenaam maakte! Pieter, Lauran, Ine, Nadine, Tim, Kristiaan, Daphna, en Veerle, ik had me geen betere kamergenoten kunnen wensen! Pieter, ge waart altijd ne super gezellige buur en heb heb me fantastisch goed geamuseerd met $u$ ! Ook hebt ge een geweldig PhD traject afgelegd. Waar je ook als postdoc zal beginnen, ik ben er zeker van dat je het nog ver gaat schoppen! Tim, Pieter vervangen als buur is geen gemakkelijke opdracht, maar je bent meer dan geslaagd!;-) Ook gij waart een super-collega! Ine, mijn carpool-maatje, het was altijd leuk om samen met $\mathrm{u}$ naar het werk te rijden. Ook al stonden we vaak ik de file, het was nooit saai onderweg. Veel succes nog met je PhD project, ik ben er zeker van dat je het tot een goed einde zal brengen! Lauran, ook bij u kon ik altijd terecht! En je was ook een ongelofelijk leuke collega! Ik wens u en Linda enorm veel geluk toe met jullie nieuwe jobs en de baby die op komst is! En ik hoop dat je ons af en toe toch nog eens komt opzoeken in het verre Hasselt! :) Nadine, met $u$ heb ik ook altijd veel plezier gehad! Veel succes nog met je project! 
Ook de andere (ex)-AlO's; Yeliz, Peggy, Laura, Marten, Emiel, Frank, Hanneke, Guus, Wino en Ellen ( $L$ ) zou ik graag willen bedanken om zo'n leuke collega's te zijn. Yeliz, I whish you all the best!!! And you know if you want to brainstorm about one of your projects; my door is always open! Peggy, veel succes nog met het afronden van je thesis en met het submitten van je manuscripten! Je werkt enorm hard en ik ben er zeker van dat alles op zijn pootjes komt!

En ook de rest van de afdeling en oud-collega's die ik nog niet vermeld heb, zou ik graag willen bedanken voor alle hulp, steun en sfeer op de afdeling: Ronit, Menno, Patrick, Chantal, Marjon, Vivian, Monique, Marjo, Danielle, Sofia, Willem, Wilma, Franky, Brigitte, Petra, Claudia, Raymond, Inge bedankt voor jullie bijdrage!

Also, I would like to thank all our collaborators! Lucie and Olivier, it wasn't easy, but without your support I never would have had such a nice chapter about cMyBP-C. Thank! Rhonda and Eric, thank you so much for giving me the chance to work in your lab! I learned so much and it were 2 fantastic months I will never forget! Thank you! Ook Boudewijn, Felix en Andreas zou ik graag willen bedanken om ervoor te zorgen dat ik onze in vitro data kon bevestigen in vivo met behulp van microPET imaging. Dankzij onze collaboratie is dit werk echt op een hoger niveau gekomen. Bedankt!

Naast al mijn collega's wil ik ook graag mijn vrienden bedanken! Bedankt allemaal voor al die leuke feestjes, terraskes en vakanties!!! Dankzij jullie kon ik mijn werk af en toe toch even thuis laten en lekker ontspannen.

Ook wil ik iedereen van mijn familie bedanken. Jullie steunden mij allemaal door dik en dun! Mama en papa bedankt om mij de kans te geven om dit alles te kunnen bereiken en altijd in mij te geloven! Ik kan dag en nacht bij jullie terecht en zou niet weten wat ik zonder jullie zou moeten beginnen! Jullie zijn echt geweldig!! Thomas, Sophie, en Lina ook jullie hebben voor vele gezellige avonden gezorgd en altijd interesse getoond in mijn werk! Dikke merci! Oma\&opa en bomma, jullie deur stond altijd open. Bedankt om er altijd voor mij te zijn en in mij te geloven! Jullie zijn een enorme steun voor mij! Herman, Dirk, ook jullie wil ik bedanken, jullie hebben me vanaf de eerste dag doen thuis voelen. Bedankt voor al je steun en betrokkenheid!

En dan als laatste, maar zeker niet onbelangrijkste, mijn allerliefste Ben. We zijn al enkele jaartjes samen en ik zou me geen leven zonder $u$ kunnen voorstellen. Jij bent er ALTIJD voor mij, 7 op 7, 24 op 24, no matter what! Ik kan voor alles bij u terecht en ook al had ik soms een slechte dag, je weet me altijd op te vrolijken en gerust te stellen. (;) We hebben enorm veel plezier samen op ons appartementje, en ben er van overtuigd dat we nog vele avonturen gaan beleven! Bedankt om de mijne te zijn!!! :- 
Curriculum Vitae 


\section{Curriculum Vitae}

Ellen Dirkx was born on May $5^{\text {th }}, 1985$ in Geel, Belgium. In 2003, she acquired her high school diploma at the Pius $X$ college Tessenderlo, Belgium. She started her bachelor education in Molecular Life Sciences at the transnational University Limburg (tUL; campus Hasselt, Belgium). For this degree she did a 6 weeks internship "intro to Bio-electronics" at the institute for Material investigation in Micro-electronics, Hasselt University, Belgium and a 2 months internship "Brain metabolism in Alzheimer disease" at the Department of Molecular cell biology, Maastricht University, The Netherlands. She finalized her bachelor study in 2006. Subsequently, she participated in the master of "Clinical Molecular Life Sciences" at Hasselt University, Belgium. For this degree, she performed an internship of 6 months at Virga Jesse Hospital (Hasselt Belgium) where she had to optimize metafase-cytogenetics and array comparative genomic hybridization on immunophenotypical plasma cell populations. In July 2007, she graduated with distinction.

In September 2007, she started her PhD program at the department of Molecular Genetics within the school of Health, Medicin and Life Sciences, under supervision of Dr. Joost Luiken, Dr. Guillaume van Eys and Prof. Dr. Jan Glatz. The topic of her research was "Genetic modulation of protein kinase-D in the heart: A novel target to restore metabolism and function in diabetic cardiomyopathy". During this time, she mentored several bachelor and master students during their internships, and she was tutor of 3th year bachelor students for 2 months. Also, she was PhD representative of the Cardiovascular Research Institute Maastricht (CARIM) for 1 year. During her 4 year PhD program, she attended all Cardiovascular PhD-training courses of the Dutch Heart Foundation and actively attended international conferences, among which the Conferences of the Society for heart and vascular metabolism (SHVM), Consortium meeting EU FP6 Integrated Project "Exgenesis", Experimental Biology meeting, and the Lipid Binding Protein Workshops. During these conferences, she was able to present her research both orally and by poster presentation. She obtained the Early investigators award at 9th Annual Conference of the Society for heart and vascular metabolism (SHVM), Brussels, Belgium, and she is selected for the Young Investigator Award at the Heart Failure Winter Research Meeting 2012, Les Diablerets, Switzerland. Furthermore, she got the price for best oral presentation at the Cardiovascular PhD-training course of the Dutch Heart Foundation, Papendal (Arnhem), 2010 and best poster presentation at the Cluster of Genetics and Cell Biology, Maastricht University, 2010. In addition, she received a Graduate/Postdoctoral Travel Award at the ASBMS 2011 Annual Meeting, held in conjunction with Experimental Biology, Washington, DC, USA. Furthermore, she started collaborations with other research groups on international level. In June 2009, 
she visited Dr. Lucie Carrier (University Medical Center Hamburg-Eppendorf, Germany) to study the functional relevance of cMyBP-C phosphorylation by PKD1 by using CMyBP-C KO mice. In the period of April-May 2010, she worked at the laboratory of Prof. Dr. Rhonda Bassel-Duby and Prof. Dr. Eric N. Olson (UTSouthwestern Medical Center, Dallas, USA) to study the effect of MEF2 deletion (using MEF2 knockout mice) on PKD1 function in cardiac metabolism. To accomplish this, she received an Albert Renold Travel Fellowship granted by the European Foundation for the Study of Diabetes.

From September 2011 until present, she started as a postdoc fellow at the department of Cardiology in Maastricht. Under supervision of Prof. Dr. Leon De Windt, she studies the role of microRNAs in heart failure. 


\section{List of publications}

- Ellen Dirkx, Robert W. Schwenk, Will A. Coumans, Nicole Hoebers, Yeliz Angin, Benoit Viollet, Arend Bonen, Guillaume J. J. M. van Eys, Jan F.C. Glatz, Joost J. F. P. Luiken. Protein kinase-D1 is essential for contractioninduced glucose uptake, but is not involved in fatty acid uptake into cardiomyocytes. J. Biol. Chem. 2011 Dec 9

- Kinet V, Dirkx E, De Windt LJ. Quaero muneris: Exploring microRNA function in cardiovascular disease. J Mol Cell Cardiol. 2011 Oct 28.

- Ellen Dirkx, Robert W. Schwenk, Jan F.C. Glatz, Joost J.F.P. Luiken, Guillaume J.J.M. van Eys. High fat diet induced diabetic cardiomyopathy. Prostaglandins Leukot Essent Fatty Acids. 2011 Nov;85(5):219-25

- Schwenk RW, Dirkx E, Coumans WA, Bonen A, Klip A, Glatz JF, Luiken JJ. Requirement for distinct vesicle-associated membrane proteins in insulinand AMP-activated protein kinase (AMPK)-induced translocation of GLUT4 and CD36 in cultured cardiomyocytes. Diabetologia. 2010 Oct;53(10):2209-19.

- Ellen Dirkx, Olivier Cazorla, Robert W. Schwenk, Ilka Lorenzen-Schmidt, Didier Vertommen, Sakthivel Sadayappan, Freek G. Bouwman, Edwin C. Mariman, Johan Van Lint, Lucie Carrier, Guillaume J.J.M. van Eys, Jan F.C. Glatz, Joost J.F.P. Luiken. Protein kinase D increases maximal $\mathrm{Ca}^{2+}$ activated tension of cardiomyocyte contraction by selective phosphorylation of CMyBP-C (resubmitted to The American Journal of Physiology)

- Ellen Dirkx, Robert W. Schwenk, Didier Vertommen, Freek G. Bouwman, Edwin C. Mariman, Jan F.C. Glatz, Joost J.F.P. Luiken, Guillaume J.J.M. van Eys. Detection of cardiac myosin binding protein-C, by a phospho-specific PKD antibody in contracting rat cardiomyocytes (resubmitted to The Journal of Physiological Sciences)

- Ellen Dirkx, Guillaume J.J.M. van Eys, Robert W. Schwenk, Laura K.M. Steinbusch, Nicole Hoebers, Ben J. Janssen, Boudewijn Brans, Andreas T. Vogg, Rhonda Bassel-Duby, Jan F.C. Glatz, Joost J.F.P. Luiken. A Protein Kinase D1-MEF2D Pathway Controls Metabolic Substrate Switching in the Failing Heart (submitted to Circulation)

- $\quad$ Robert W. Schwenk, Yeliz Angin, Laura K.M. Steinbusch, Ellen Dirkx, Nicole Hoebers, Will A. Coumans, Arend Bonen, Jos L.V. Broers, Guillaume J.J.M. van Eys, Jan F.C. Glatz, Joost J.F.P. Luiken. Overexpression of VAMP3 protects against lipid-induced insulin resistance in cardiomyocytes (submitted to Diabetes)

- Yeliz Angin ${ }^{1}$, Laura K.M. Steinbusch ${ }^{1}$, Peter J. Simons, Kim Douma, Sabrina Greulich, Nicole T.H. Hoebers, Will A. Coumans, Wino Wijnen, Ellen Dirkx, Michaela Diamant, D. Margriet Ouwens, Jan F.C. Glatz, Joost J.F.P. Luiken. 
CD36 inhibition prevents lipid accumulation and contractile dysfunction in rat cardiomyocyte cultures (submitted to Cell. mol. life sciences)

- Laura L.K.M. Steinbusch ${ }^{1}$, Ellen Dirkx ${ }^{1}$, Will A. Coumans, Nicole T.H. Hoebers Jan F.C. Glatz, Joost J.F.P. Luiken. Both PKD1 and AMPK are essential in cardiac GLUT4-mediated glucose uptake during contraction (in preparation)

- $\quad$ Ellen Dirkx, An Thys, Guillaume J.J.M. van Eys, Jan F.C. Glatz, Joost J.F.P. Luiken. Phosphatidylinositol 4-Kinase selectively leads to GLUT4-mediated glucose uptake and not CD36-mediated fatty acid uptake in contracting cardiomyocytes (in preparation)

- Laura K.M. Steinbusch, Yeliz Angin, Robert Schwenk, Ellen Dirkx, Kim Douma, Will A. Coumans, Michaela Diamant, Jan F.C. Glatz, Joost J.F.P. Luiken, D.Margriet Ouwens. Regulation of endosomal $\mathrm{pH}$ is disturbed in cardiomyocytemodels for lipid-induced insulin resistance (in preparation)

- JayT. McFarlan , I Momken, Ellen Dirkx , An Thys, Jan F.C. Glatz, Joost J.F.P, Luiken, Arend Bonen. Leptin stimulation of fatty acid oxidation is dependent on the fatty acid transporter CD36 (in preparation)

- $\quad$ el Azzouzi $H^{1}$, Leptidis $\mathrm{S}^{1}$, Dirkx E, da Silva GJ, Bitsch N, van der Nagel R, Olieslagers S, Kisters N, van Bilsen M, Luiken JJ, Schrauwen P, da Costa Martins PA, De Windt LJ. The miR-199a/-214 cluster targets the PPARbetaPGC1-alpha complex and provokes maladaptive alterations of the mitochondrial beta-oxidation capacity in heart failure (in preparation) 


\section{Oral presentations}

- May 22th, 2011: 9th Annual Conference of the Society for heart and vascular metabolism (SHVM), Brussels, Belgium (Early investigator award) Title: Protein kinase-D1 overexpression in mice prevents lipid-induced insulin resistance and cardiomyopathy by upregulation of glucose uptake

- April 12 $2^{\text {th }}, 2011$ : Experimental Biology, ASBMB annual meeting, Washington, DC, USA

Title: Protein kinase-D1 overexpression in mice prevents lipid-induced insulin resistance and cardiomyopathy by upregulation of glucose uptake (Awarded with a full bursary)

- October $15^{\text {th }}, 2010$ : Cardiovascular PhD-training course of the Dutch Heart Foundation, Arnhem, the Netherlands.

Title: Protein kinase-D1 overexpression in mice prevents lipid-induced insulin resistance and cardiomyopathy by upregulation of glucose uptake (awarded for best oral presentation)

- May 12 $12^{\text {th }}, 2009$ : Consortium meeting EU FP6 Integrated Project "Exgenesis", Paris, France

Title: Regulation of myocardial substrate uptake by protein kinase D

- July $1^{\text {st }}, 2008$ : 6 th Annual Conference of the Society for heart and vascular metabolism, Boston, USA

Title: A role for protein kinase $D$ in the contraction signaling pathway, independent of AMPK

- April $8^{\text {th }}, 2008$ Consortium meeting EU FP6 Integrated Project "Exgenesis", Padova, Italy

Title: Identification of a downstream substrate of protein kinase $D$ in contraction signaling 


\section{Poster presentations}

- January 18-21, 2012: Heart Failure Winter Research Meeting, Les Diablerets, Switzerland

Title: A Protein Kinase D1-MEF2D Pathway Controls Metabolic Substrate Switching in the Failing Heart

- April 11 ${ }^{\text {th }}, 2011$ : Experimental Biology, ASBMB annual meeting, Washington, DC, USA

Title: Protein kinase-D1 overexpression in mice prevents lipid-induced insulin resistance and cardiomyopathy by upregulation of glucose uptake (Awarded with a travel fellowship)

- January $20^{\text {th }}, 2010$ Cluster of Genetics and Cell Biology, Maastricht University

Title: Protein kinase-D1 overexpression in mice prevents lipid-induced insulin resistance and cardiomyopathy by upregulation of glucose uptake (Best poster presentation)

- August 23-26, 2009: 7th Annual Conference of the Society for heart and vascular metabolism (SHVM), Padova, Italy

Title: ROS-induced activation of protein kinase D increases GLUT4mediated glucose uptake but not CD36-mediated fatty acid uptake by contracting cardiomyocytes

- June 2-4, 2009: 14th International Conference Biochemistry of Exercise, University of Guelph, Guelph, Ontario, Canada

Title: Cardiac myosin binding protein $C$ is a direct downstream target of protein kinase $D$ in contraction signaling

\section{Fellowships/Awards}

- $\quad$ Selected for the Young Investigator Award: January 18-21, 2012, Heart Failure Winter Research Meeting, Les Diablerets, Switzerland

- Early investigator award: May 22th, 2011, granted by the Society for heart and vascular metabolism (SHVM), Brussels, Belgium

- Albert Renold Travel Fellowship: February $31^{\text {st }}, 2011$, granted by the European Foundation for the Study of Diabetes

- Graduate/Postdoctoral Travel Award: January 28 ${ }^{\text {th }}, 2011$ ASBMS Annual Meeting, held in conjunction with Experimental Biology, Washington, DC, USA

- Best Oral Presentation: October 11-15, 2010 Cardiovascular PhD-training course of the Dutch Heart Foundation, Papendal (Arnhem)

- Best Poster Presentation: January 20 $0^{\text {th }}, 2010$ Cluster of Genetics and Cell Biology, Maastricht University 